Florida International University FIU Digital Commons

FIU Electronic Theses and Dissertations

University Graduate School

$11-1-2007$

\title{
Durability Assessment of Polymer Trileaflet Heart Valves
}

Siobhain Lynn Gallocher

Florida International University, sgallocher@yahoo.com

DOI: $10.25148 /$ etd.FI08081519

Follow this and additional works at: https://digitalcommons.fiu.edu/etd

\section{Recommended Citation}

Gallocher, Siobhain Lynn, "Durability Assessment of Polymer Trileaflet Heart Valves" (2007). FIU Electronic Theses and Dissertations. 54.

https://digitalcommons.fiu.edu/etd/54

This work is brought to you for free and open access by the University Graduate School at FIU Digital Commons. It has been accepted for inclusion in FIU Electronic Theses and Dissertations by an authorized administrator of FIU Digital Commons. For more information, please contact dcc@fiu.edu. 


\section{FLORIDA INTERNATIONAL UNIVERSITY}

Miami, Florida

\section{DURABILITY ASSESSMENT OF POLYMER TRILEAFLET HEART VALVES}

A dissertation submitted in partial fulfillment of the

requirements for the degree of

DOCTOR OF PHILOSOPHY

in

BIOMEDICAL ENGINEERING

by

Siobhain Lynn Gallocher

2007 
To: Interim Dean Amir Mirmiran

College of Engineering and Computing

This dissertation, written by Siobhain Lynn Gallocher, and entitled Durability Assessment of Polymer Trileaflet Heart Valves, having been approved in respect to style and intellectual content, is referred to you for judgment.

We have read this dissertation and recommend that it be approved.

Anthony McGoron

Eric Crumpler

Kuang-Hsi Wu

Michael Christie

Richard T. Schoephoerster, Major Professor

Date of Defense: November 1, 2007

The dissertation of Siobhain Lynn Gallocher is approved.

$\begin{array}{r}\text { Interim Dean Amir Mirmiran } \\ \text { College of Engineering and Computing } \\ \hline \begin{array}{r}\text { Dean George Walker } \\ \text { University Graduate School }\end{array}\end{array}$

Florida International University, 2007 
C Copyright 2007 by Siobhain Lynn Gallocher

All rights reserved. 


\section{DEDICATION}

I dedicate this dissertation to my husband, Marcus. I finally see the light at the end of this tunnel. I thank you for your humor and support; it is because of you that I have been able to persevere. 


\section{ACKNOWLEDGMENTS}

Firstly, I would like to thank Dr. Richard Schoephoerster. I was fortunate enough to get the opportunity to work in his lab since I entered Florida International University as a Bachelor's student. My experience has helped shape me into the researcher I am now, and I know that all I have learned can be applied to my future experiences.

A special thanks to the group at Innovia, LLC. My research was funded in part by a National Heart, Lung, and Blood Institute SBIR Phase II HL0704010 to Innovia, LLC. In developing this project, I have had the opportunity to refer to the expertise of Len Pinchuk, Marc Ramer, John Martin, Yasushi P. Kato, Scott Arp, Bruce Weber, and Saul Gottlieb. Their advice has helped me fine-tune my research, and John, Saul, and Scott have manufactured all fixtures and components that were found necessary.

Thank you to all the people I have worked with in the Biomedical Engineering Department: in particular, Rosa Ramirez, who helped with all photogrammetry data analysis; Thomas Claiborne, who put together the MatLab code for hydrodynamic data analysis; Dr. James Byrne, who helped set up the photogrammetry equipment; and Qiang Wang, who has been a true friend and helped me at any and every opportunity.

I am indebted to Amit Datye. His ABAQUS expertise has helped me when I did not think there was any solution to the problems I was having. He was available at all hours for every ridiculous-seeming question I had to ask.

Finally, I would like to finish by thanking my committee members, Dr. Anthony McGoron, Dr. Eric Crumpler, Dr. Kuang-Hsi Wu, and Dr. Michael Christie, for their guidance throughout this whole ordeal. 


\title{
ABSTRACT OF THE DISSERTATION \\ DURABILITY ASSESSMENT OF POLYMER TRILEAFLET HEART VALVES
}

by

\author{
Siobhain Lynn Gallocher
}

Florida International University, 2007

Miami, Florida

Professor Richard T. Schoephoerster, Major Professor

The durability of a polymer trileaflet valve is dependent on leaflet stress concentrations, so valve designs that reduce stress can, hypothetically, increase durability. Design aspects that are believed to contribute to reduced leaflet stress include stent flexibility, parabolic coaptation curvature, and leaflet anisotropy. With this in mind, the purpose of this investigation was to elucidate what specific combinations of these parameters promote optimal acute and long-term valve function.

A combination of four stent designs, seven leaflet reinforcement materials, and three coaptation geometries were evaluated through a combination of experimentation and modeling. Static tensile and Poisson's ratio tests and dynamic tensile fatigue testing were used to evaluate the individual leaflet components; and hydrodynamic testing and accelerated valve fatigue was used to assess complete valve prototypes. The two most successful designs included a $0.40 \mathrm{~mm}$ thick knit-reinforced valve with a fatigue life of 10.35 years, and a $0.20 \mathrm{~mm}$ thick knit-reinforced valve with a $28.9 \mathrm{mmHg}$ decrease in pressure drop over the former.

A finite element model was incorporated to verify the impact of the abovementioned parameters on leaflet stress concentrations. Leaflet anisotropy had a large 
impact on stress concentrations, and matching the circumferential modulus to that of the natural valve showed the greatest benefit. Varying the radial modulus had minimal impact. Varying coaptation geometry had no impact, but stent flexibility did have a marked effect on the stress at the top of the commissure, where a completely rigid stent resulted in a higher peak stress than a flexible stent $(E=385 \mathrm{MPa})$.

In conclusion, stent flexibility and leaflet anisotropy do effect stress concentrations in the SIBS trileaflet valve, but coaptation geometry does not. Regions of high stress concentrations were linked to failure locations in vitro, so a fatigue prediction model was developed from the $\mathrm{S} / \mathrm{N}$ curves generated during dynamic tensile testing of the $0.20 \mathrm{~mm}$ knit-reinforced leaflets. Failure was predicted at approximately 400 million cycles (10 years) at the top of the commissure. In vitro fatigue of this valve showed failure initiation after approximately 167 million cycles (4.18 years), but it was related to a design defect that is subsequently being changed. 


\section{TABLE OF CONTENTS}

CHAPTER

PAGE

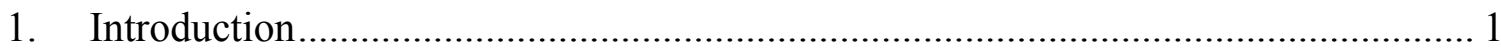

1.1. General Statement of Problem Area .............................................................. 1

1.2. Research Purpose ………………………………....................................... 3

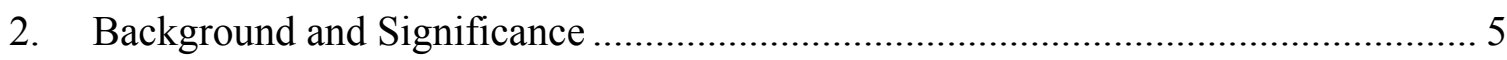

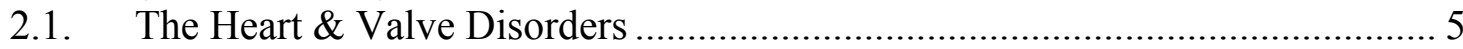

2.2. Commercially Available Prosthetic Valves ......................................................... 6

2.2.1. Mechanical Valves............................................................................

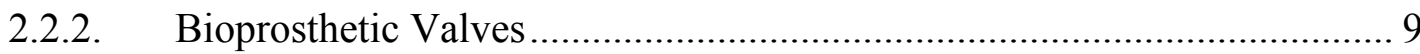

2.3. Polymer Trileaflet Valves .............................................................................. 11

2.4. Design Criteria: The Natural Aortic Valve as a Model ................................... 15

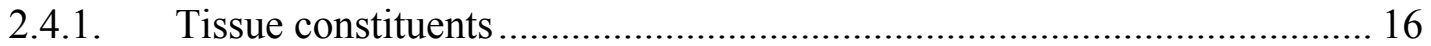

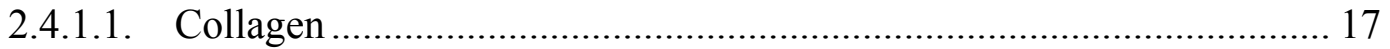

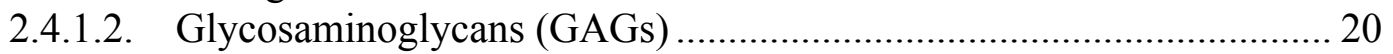

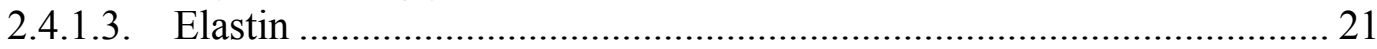

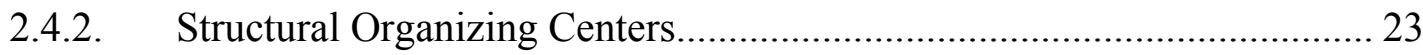

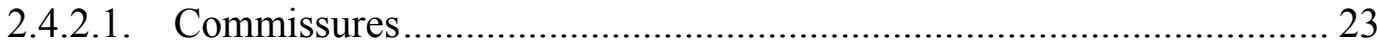

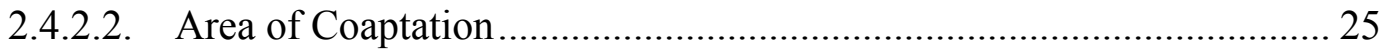

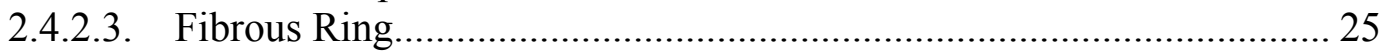

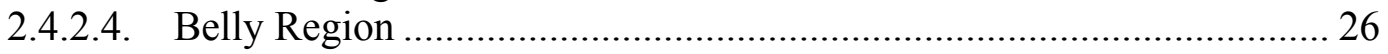

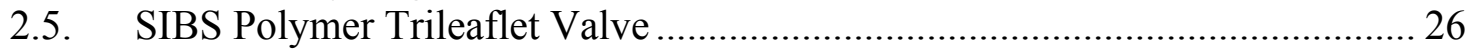

2.6. Valve Characterization Methods................................................................... 31

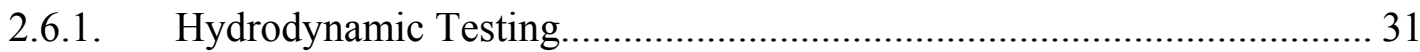

2.6.2. Accelerated Fatigue Testing ……………................................................ 36

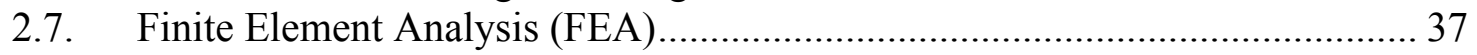

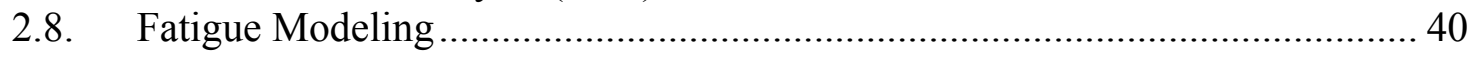

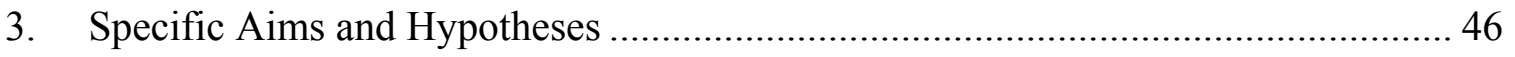

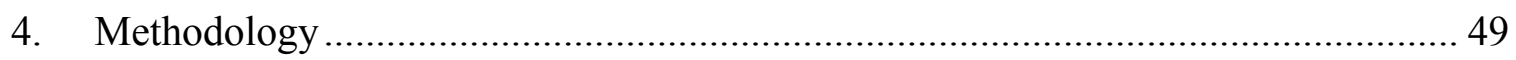

4.1. Valve Manufacturing Procedure ……………......................................... 50

4.1.1. Design 1: Pre-formed individual leaflets ................................................. 50

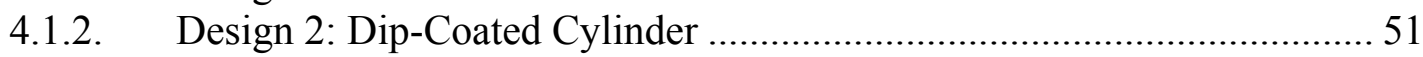

4.1.3. Design 3: Compression-Molded Cylinder ................................................. 54

4.1.4. Design 4: Solvent Cast Cylinder.......................................................... 55

4.2. Specific Aim 1 - Valve characterization ................................................... 58

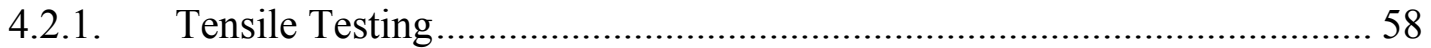

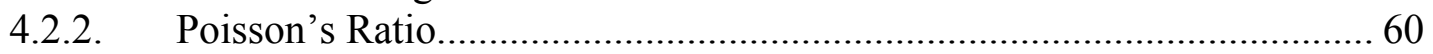

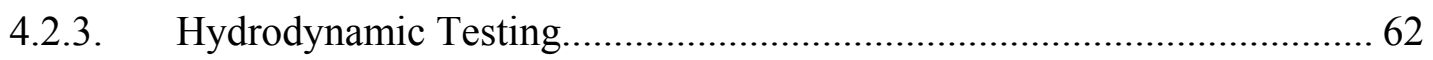

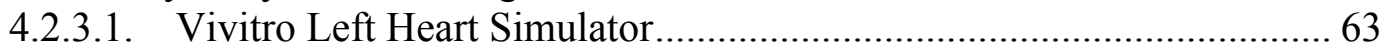

4.2.3.2. Measurements and Outcome Measures ................................................. 64 


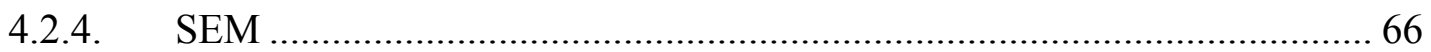

4.3. Specific Aim 2 - Finite Element Modeling …….......................................... 67

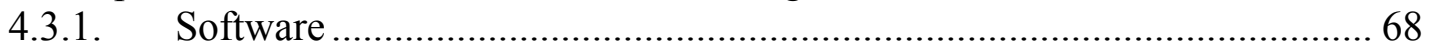

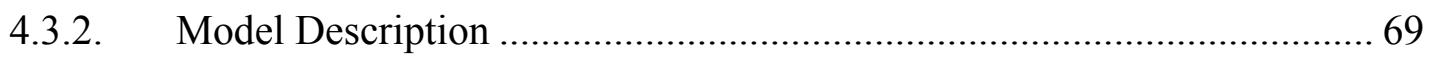

4.3.3. Boundary Conditions …………………................................................. 73

4.3.4. Material Properties............................................................................... 77

4.3.4.1. Material Orientation and Coordinate System ..................................... 79

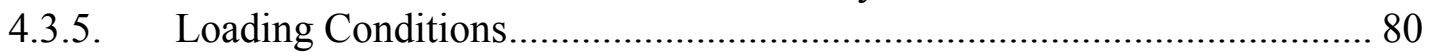

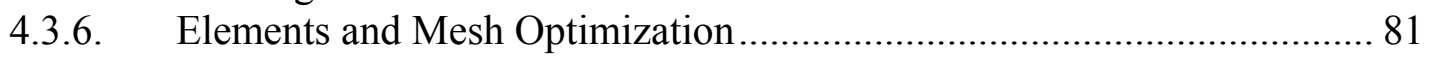

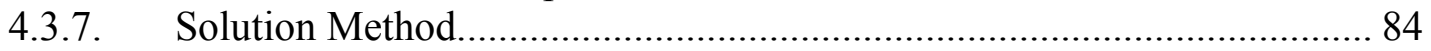

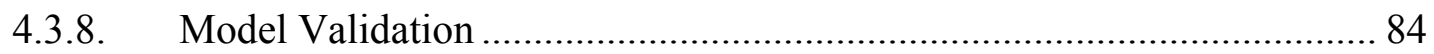

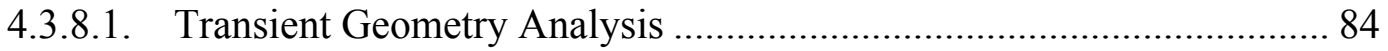

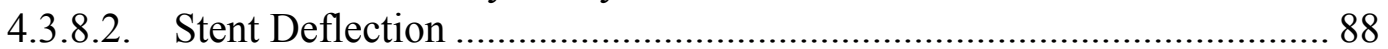

4.4. Specific Aim 3 - Durability Assessment ...................................................... 90

4.4.1. Accelerated Fatigue Testing ………………….................................... 90

4.4.1.1. Cyclic Tensile Testing ................................................................... 90

4.4.1.2. Valve Durability Testing................................................................... 91

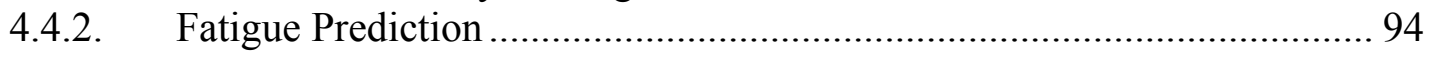

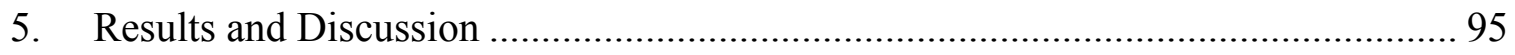

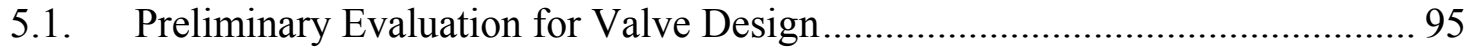

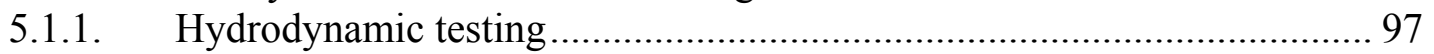

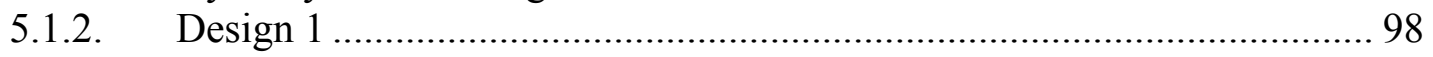

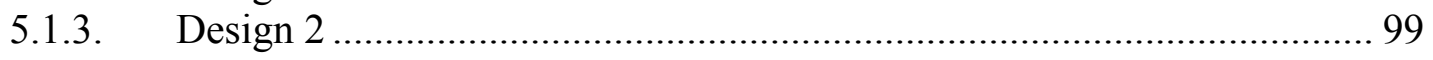

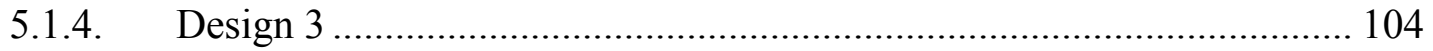

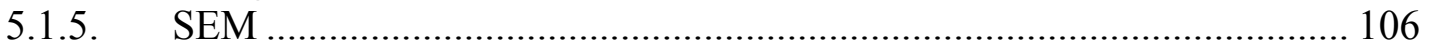

5.2. Design Refinement and Quantification for Improved Durability ................... 108

5.2.1. Leaflet Design and Manufacture.......................................................... 109

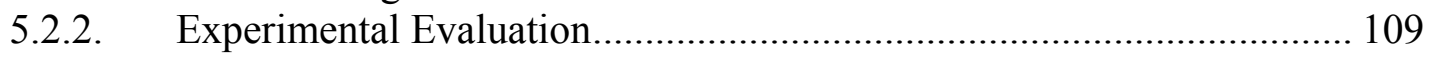

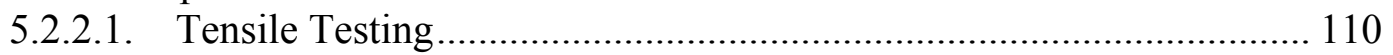

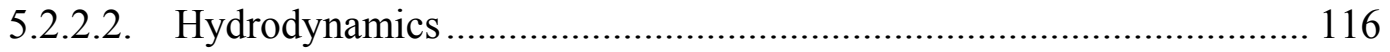

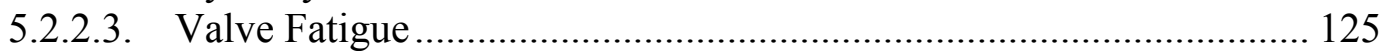

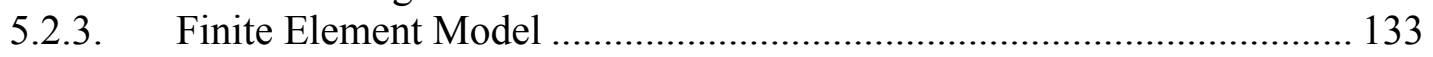

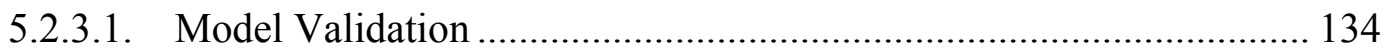

5.2.3.2. Material Properties........................................................................... 139

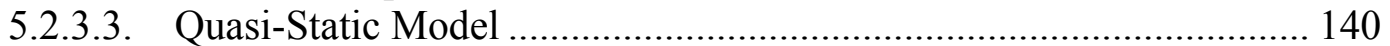

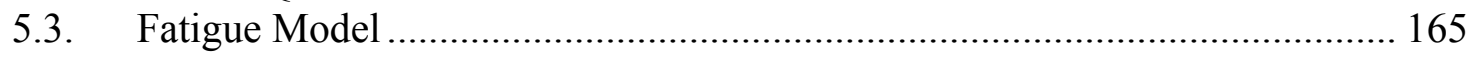

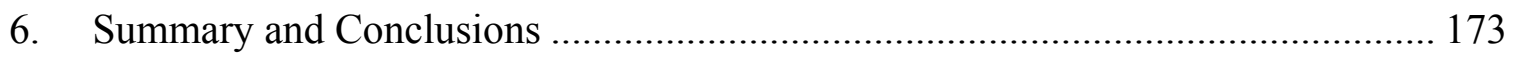

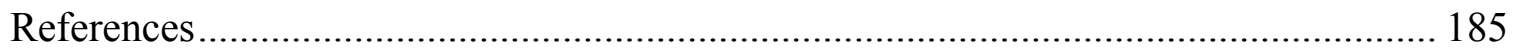

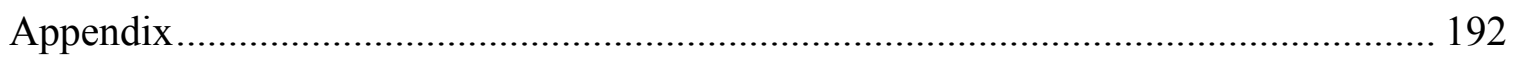

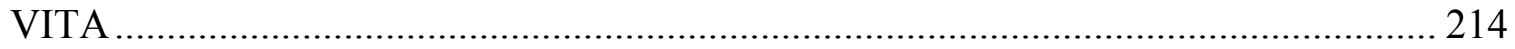




\section{LIST OF TABLES}

TABLE

PAGE

Table 1. Minimum performance requirements for aortic valve prostheses (ISO 5840:2005).

Table 2. Listing of all potential candidates for manufacture and testing of a trileaflet heart valve

Table 3. SIBS valve design summary

Table 4. Valve leaflet and stent components that were subjected to tensile testing (refer to section 4.1 for the valve manufacturing procedure).

Table 5. Summary of valves subjected to hydrodynamic testing (refer to section 4.1 for the valve manufacturing procedure).

Table 6. Specifications of cardiac outputs and heart rates for hydrodynamic testing of heart valves.

Table 7. Summary of valve models. Refer to section 4.1 for the valve models that represent experimental prototypes, and refer to Figure 16 - Figure 19 for the specific valve geometries.

Table 8. A summary of the boundary conditions for all valve models. A total of 13 boundary conditions was instituted.

Table 9. Summary of uncoated and coated thicknesses of the dip coated Dacron and all samples manufactured by solvent casting. The precision of the digital calipers is provided.

Table 10. Engineering and true Young's moduli for fiber-reinforced leaflet materials, isotropic leaflet material, and isotropic stent materials in the circumferential direction, radial direction, and $45^{\circ}$ in between.

Table 11. Summary of mean transvalvular pressure drop during forward flow, percentage regurgitation, effective orifice area $\left(\mathrm{A}_{\mathrm{EO}}\right)$, and leaflet thickness for all valves subjected to hydrodynamic testing. Values for the pressure and regurgitation were averaged over all cardiac output and heart rate combinations, and $\mathrm{A}_{\mathrm{EO}}$ was measured at $70 \mathrm{BPM}, 5.6 \mathrm{~L} / \mathrm{min}$.

Table 12. Summary of peak pressure gradient, mean pressure gradient, and $A_{E O}$ for the BARD reinforced valve and a selection of commercially available aortic valve prostheses [76].

Table 13. Summary of the primary valve designs subjected to fatigue evaluation. 
Table 14. Validation of the quasi-static FEM. The particular valve included the BARD-reinforced leaflets with slight coaptation curvature. These leaflets were mounted on the medium profile, $48.5 \%$ styrene stent.

Table 15. Orthotropic material engineering constants used as input into the quasi-static FEM. Note all Poisson's ratio values were assumed to be zero.

Table 16. Isotropic material constants used as input into both the quasi-static and dynamic FEMs.

Table 17. Predicted failure stresses in the circumferential and radial directions for specific cyclic intervals from 1 to 400 million cycles.

Table 18. Qualitative valve failure rating 


\section{LIST OF FIGURES}

FIGURE

PAGE

Figure 1. A diagrammatic frontal section through the heart, showing major landmarks and the path of blood flow [17].

Figure 2. Three basic types of mechanical heart valves: (a) Ball and cage valve,

(b) Tilting disk valve, and (c) Bileaflet valve (http://www.edwards.com/PatientsandFamilies/MyHeart/ClinicalProcedures/ ....... 7

Figure 3. Three types of bioprosthetic heart valves: (a) Stented porcine valve, (b) Stented bovine pericardial valve, (c) Stentless porcine valve (http://www.edwards.com/PatientsandFamilies

/MyHeart/ClinicalProcedures/ValveReplacementEU.aspx)

Figure 4. A cutaway through the leaflet and aortic wall showing the internal configuration of the fibrosa, spongiosa, and ventricularis. Adapted from Vesely $[32]$

Figure 5. Typical collagen fiber architecture in the aortic valve leaflet [37]. The commissures are denoted by 'c'.

Figure 6. Polyisobutylene backbone showing alternating quaternary and secondary carbon linkages that do not have sites to oxidize.

Figure 7. Composite SIBS polymer trileaflet valve designs: (a) Polypropylene embedded spherical valve, (b) polypropylene embedded elliptical valve, (c) LARS $^{\circledR}$ mesh embedded spherical valve, (d) Dacron embedded thermal-form valve.

Figure 8. Hydrodynamic function tester for prosthetic heart valves is shown. The fluid mechanical environment is monitored with direct pressure transducers and flow probes.

Figure 9. Representative flow waveform where (1) represents the closing volume, (2) represents leakage volume, and (3) represents the stroke volume (ISO 5840:2005).

Figure 10. Simultaneous tracings of the left ventricular and aortic pressure waveforms (math.bd.psu.edu/faculty/stevens/PVpresentation.ppt).....

Figure 11. Casting plate for the uniform coating of leaflet reinforcement fabrics 
Figure 12. SEM images of a solvent cast PET weave sample showing the crosssectional and surface views. It is apparent from the cross-sectional view that the fiber reinforcement is embedded in the center of the SIBS matrix, and that the exposed polymer surface is smooth with no fiber exposure

Figure 13. SIBS specimen ( $8.5 \%$ styrene) mounted on the ELF tester showing the location of the four fiducial points at (a) $\varepsilon=0 \mathrm{~mm} / \mathrm{mm}$ and (b) $\varepsilon=0.15 \mathrm{~mm} / \mathrm{mm}$. A $1 \times 1 \mathrm{~mm}$ grid is shown overlaying the markers to quantify longitudinal and transverse strain. In Photoshop ${ }^{\circledR}$ each one millimeter grid is subdivided into 5 segments, providing a measurement precision of $0.2 \mathrm{~mm}$. A particularity of each point was used to identify its location at both strain locations.

Figure 14. Schematic of the left heart and systemic circulation simulator: stroke volume $0-135 \mathrm{~mL}$, aortic root compliance air volume $0-585 \mathrm{~mL}$, characteristic compliance air volume $0-650 \mathrm{~mL}$, valve mounting up to size $33 \mathrm{~mm}$, blood analog fluid $2 \mathrm{~L}$

Figure 15. Typical pressure and flow waveforms showing (a) left ventricular pressure $(\mathrm{mmHg})$, (b) aortic pressure $(\mathrm{mmHg})$, (c) aortic flow $(\mathrm{L} / \mathrm{min})$, and camera trigger.

Figure 16. Geometry for models showing the a) top view and b) side view of the high profile stent with "slight curve" leaflet curvature.

Figure 17. Geometry for models showing the a) top view and b) side view of the medium profile stent with "slight curve" leaflet curvature.

Figure 18. Geometry for models showing the a) top view and b) side view of the medium profile stent with "flat" leaflet curvature.

Figure 19. Geometry for models showing the a) top view and b) side view of the medium profile stent with "larger curve" leaflet curvature.

Figure 20. Schematic of the (I) whole polymer trileaflet valve and (II) one third of the valve with interior detail. Labeled segments represent the (a) stent, (b) area of coaptation, (c) commissure, (d) leaflet belly, e) stent-leaflet joint. Embedded in the polymer is a reinforcement fabric....

Figure 21. Image showing the contact pairs between (a) a leaflet and its corresponding stent and (b) adjacent stents. The tie constraint establishes a bond between adjacent stent thirds and between the contacting leaflet and stent sections. This bond ensures that the nodes in closest proximity are restricted to move as one. 
Figure 22. Image showing the contact interaction pairs between a) coapting leaflet segments and b) leaflet-stent contacting regions during systole. The contact constraint alows the definition of the tangential frictional behavior between two contacting surfaces.

Figure 23. Image showing the fixed boundary condition applied to the base of the stent. A fixed boundary condition means that that base of the stent is restricted from either translating or rotating, making it a completely rigid restraint.

Figure 24. Model of valve showing the global coordinate system location at the highest central point of the valve.

Figure 25. Individual leaflet showing the location of the local coordinate system and the orientation of the 1-, 2-, and 3(n)-directions.

Figure 26. Characteristic aortic, ventricular, and transvalve pressure waveforms for a BARD reinforced SIBS trileaflet valve.

Figure 27. BARD-reinforced SIBS valve with medium coaptation curvature and $48.5 \%$ styrene stent. Image shows (a) top and (b) oblique view including the leaflet marked with approximately 120 fiducial points.

Figure 28. Image acquisition set-up showing (a) how the borescopes are inserted into the acrylic endoscope mounting chamber, (b) the orientation of each camera and associated borescope, (c) the location of the valve with respect to each borescope, and (c) the image generation on the imaging PC....

Figure 29. Schematic of the image acquisition system for imaging valves undergoing hydrodynamic testing

Figure 30. Quantitative validation of point-to-point distances between the experimental DCSP method and the finite element model. Points were selected on the image, and the distances between each was computed from the knowledge of their 3-D coordinates. The corresponding points were selected on the corresponding finite element model (right), and the distances between each were computed for comparison.

Figure 31. Photoshop ${ }^{\circledR}$ image showing grid overlay and stent post dimension lines.

Figure 32. Vivitro Systems Hi-Cycle including schematic of chamber where valve is seated.

Figure 33. Vivitest software for the acquisition, analysis, and display of heart valve test data showing the inflow, outflow, and transvalve pressure, where the peak transvalve pressure is at $90 \mathrm{mmHg}$ 
Figure 34. Mean transvalvular pressure drop during forward flow for SIBS valve designs 1,2 , and 3 .

Figure 35. Percent regurgitation measured while the valves are closed and closing for SIBS valve designs 1,2 , and 3 .

Figure 36. Design 1 valve after an equivalent of 0.628 years of cycling. Failure was as a result of leaflet-stent detachment and polymer blowout from between the mesh interstices

Figure 37. Varying iterations of the dip-coated thermal-formed design 2 valves including different polyester reinforcement fabric: (a) LARS ${ }^{\circledR}$ reinforcement mesh failure mode was polymer blowout from between mesh interstices at an equivalent of 0.908 yrs, (b) PET weave failure mode was weave degradation at an equivalent of 0.943 years, and (c) athletic mesh failure mode was polymer and mesh degradation at an equivalent of 1.38 years.

Figure 38. Dacron reinforced valve with maximum stretch oriented circumferentially. Note the surface deposits on the leaflet that resulted in a 3-fold increase in transvalvular pressure drop at 10.35 years.

Figure 39. SEM images of the Dacron-reinforced valve shown (a) prior to cycling, (b) after cycling an equivalent 10.35 years, and (c) after cycling an equivalent 10.35 years and subsequent polymer dissolution. The silicone deposits are denoted by the white arrows.

Figure 40. Stress-strain relationship in the radial and circumferential direction for (a) natural porcine valve leaflets (adapted from Li et al. [50]) and (b) dip-coated JSI leaflets with maximum elongation oriented circumferentially.

Figure 41. Dacron-reinforced valve with maximum stretch oriented in the radial direction. The failure mode was a combination of stent fracture (equivalent 2.72 years) and hole formation (equivalent 6.12 years) in the belly of the leaflet as a result of unphysiologically high stress in the circumferential direction.

Figure 42. SEM image of aortic leaflet surface of a Dacron cylinder valve dipcoated in a porous $16 \%$ styrene SIBS solution. On the right image, cracking is seen after cycling an equivalent of 5.06 years. The sketch identifies the location of the crack formation. A white arrow points to some instances of crack formation.

Figure 43. Dacron-reinforced compression molded valve showing failure due to tear formation at the center of coaptation at an equivalent of 0.635 years.

Figure 44. SEM of cross section of compression-molded leaflets. Note how the reinforcement fabric is crushed between two layers of polymer. 
Figure 45. Dacron reinforced hybrid valve showing failure due to tear initiation at the heat sealed joint at an equivalent of 1.5 years.

Figure 46. SEM images of the leaflet free edge and aortic surface of SIBS valve designs 1, 2, and 3. Samples were excised from freshly manufactured valves. The white arrows denote exposed polyester fibers. At the leaflet free edge, exposed fibers are expected due to how the edge is cut and exposed, but the leaflet surface should not have exposed fibers.

Figure 47. SEM images of the leaflet free edge and aortic surface of SIBS valve designs 2, and 3. Samples were excised from valves subjected to accelerated fatigue testing for an equivalent of 1.25 years. The white arrows denote exposed polyester fibers. Note the increase in fiber exposure on both leaflet surfaces and the fraying of fibers at the free edge.

Figure 48. Circumferential stress versus strain curve for all fiber-reinforced leaflet materials $\mathrm{N}=5$ for all samples.

Figure 49. Radial stress versus strain curves for all fiber-reinforced leaflet materials. $\mathrm{N}=5$ for all samples.

Figure 50. Circumferential stress versus strain curves for all fiber-reinforced materials up to $10 \%$ strain. $\mathrm{N}=5$ for all samples.

Figure 51. Radial stress versus strain curves for all fiber-reinforced materials up to $10 \%$ strain. $\mathrm{N}=5$ for all samples.

Figure 52. Stress versus strain curve for isotropic, non-reinforced 8.5\% SIBS i.e. the version of SIBS used in leaflet production. $\mathrm{N}=5$ for all samples....

Figure 53. Stress versus strain curve for isotropic, non-reinforced $8.5 \%$ SIBS, PET weave-reinforced 8.5\% SIBS, and XA-47 - reinforced $8.5 \%$ SIBS. $N=5$ for all samples.

Figure 54. Stress versus strain curves for isotropic, non-reinforced $30 \%$ and $48.5 \%$ SIBS i.e. the versions of SIBS used stent production. $\mathrm{N}=5$ for all samples.

Figure 55. Transvalvular pressure drop during forward flow for all prototype valves mounted on the $48.5 \%$ styrene stent. The results for the $19 \mathrm{~mm}$ TAD Magna ${ }^{\circledR}$ valve are provided for comparison. Error bars denote standard deviation between the three repeated measures.

Figure 56. Percentage regurgitation flow for all prototype valves mounted on the $48.5 \%$ styrene stent. The results for the $19 \mathrm{~mm}$ TAD Magna ${ }^{\circledR}$ valve are provided for comparison. Error bars denote standard deviation between the three repeated measures. 
Figure 57. Leaflet thickness versus mean transvalvular pressure drop for all prototype valves.

Figure 58. Circumferential and radial Young's modulus versus mean percent regurgitation for all prototype valves.

Figure 59. Circumferential and radial Young's modulus versus mean transvalvular pressure drop for all prototype valves.

Figure 60. NZ-11 valve showing tear formation along the leaflet-stent zone after an equivalent of 0.20 years of cycling. Tear viewed from the a) aortic and b) ventricular orientation.....

Figure 61. NZ-11 valve showing tear formation at the center of coaptation of the same leaflet as above.

Figure 62. XA-47 valve showing a) stent fracture, b) tear formation at the coaptation, c) polymer blowout and d) delamination at the coaptation.

Figure 63. PET Weave valve showing a) tear formation at the commissure and b) leaflet prolapse and free edge fraying...

Figure 64. BARD valve showing deterioration of the heat-sealed joint.

Figure 65. Plot of fatigue life versus the radial and circumferential moduli.

Figure 66. Hydrodynamic data showing the flow and pressure waveforms. The dashed lines $(1-8)$ represent the specific locations during the cardiac cycle where each image pair was triggered.

Figure 67. Photogrammetry image pairs corresponding to the baseline image where the valve was under a no load condition.

Figure 68. MatLab regeneration of fiducial points from the $\mathrm{x}, \mathrm{y}$, and $\mathrm{z}$ coordinated generated in Photomodeler ${ }^{\circledR}$ from Figure 67.

Figure 69. Photogrammetry image pairs corresponding to Frame 5 in Figure 66.

Figure 70. MatLab regeneration of fiducial points from the $\mathrm{x}, \mathrm{y}$, and $\mathrm{z}$ coordinated generated in Photomodeler ${ }^{\circledR}$ from Figure 69.

Figure 71. Photogrammetry image pairs corresponding to Frame 7 in Figure 66..........136

Figure 72. MatLab regeneration of fiducial points from the $x, y$, and $z$ coordinated generated in Photomodeler ${ }^{\circledR}$ from Figure 71.

Figure 73. Diastolic pressure loading regimen for the quasi-static model 
Figure 74. BARD-reinforced leaflets with slight coaptation curvature mounted on a $48.5 \%$ styrene, medium profile stent (BARD-Reg). Views include (a) top view of aortic surface stresses, (b) top view of ventricular surface stresses, (c) front view of one third of the valve showing aortic surface stresses, and (d) front view of one third of the valve showing the ventricular surface stresses.

Figure 75. BARD-reinforced leaflets with slight coaptation curvature mounted on a rigid, medium profile stent (BARD-Reg-Rigid). Views include (a) top view of aortic surface stresses, (b) top view of ventricular surface stresses, (c) front view of one third of the valve showing aortic surface stresses, and (d) front view of one third of the valve showing the ventricular surface stresses.

Figure 76. BARD-reinforced leaflet with flat coaptation curvature mounted on a $48.5 \%$ styrene, medium profile stent (BARD-Flat). Views include (a) top view of aortic surface stresses, (b) top view of ventricular surface stresses, (c) front view of one third of the valve showing aortic surface stresses, and (d) front view of one third of the valve showing the ventricular surface stresses.

Figure 77. BARD- reinforced leaflet with large coaptation curvature, mounted on a $48.5 \%$ styrene, medium profile stent (BARD-Lrg). Views include (a) top view of aortic surface stresses, (b) top view of ventricular surface stresses, (c) front view of one third of the valve showing aortic surface stresses, and (d) front view of one third of the valve showing the ventricular surface stresses.

Figure 78. SIBS-leaflet with slight coaptation curvature mounted on a $48.5 \%$ styrene, medium profile stent (SIBS-Reg). Views include (a) top view of aortic surface stresses, (b) top view of ventricular surface stresses, (c) front view of one third of the valve showing aortic surface stresses, and (d) front view of one third of the valve showing the ventricular surface stresses.

Figure 79. JSI-reinforced valve with slight coaptation curvature mounted on a $48.5 \%$ styrene, medium profile stent (JSI-Reg). Views include (a) top view of aortic surface stresses, (b) top view of ventricular surface stresses, (c) front view of one third of the valve showing aortic surface stresses, and (d) front view of one third of the valve showing the ventricular surface stresses.

Figure 80. JSI-reinforced leaflet (max compliance oriented circumferentially) with slight coaptation curvature mounted on a $30 \%$ styrene, high profile stent (JSI-HiP-Circ). Views include (a) top view of aortic surface stresses, (b) top view of ventricular surface stresses, (c) front view of one third of the valve showing aortic surface stresses, and (d) front view of one third of the valve showing the ventricular surface stresses. 
Figure 81. JSI-reinforced leaflet (max compliance oriented radially) with slight coaptation curvature mounted on a $30 \%$ styrene, high profile stent (JSI-HiP-Rad). Views include (a) top view of aortic surface stresses, (b) top view of ventricular surface stresses, (c) front view of one third of the valve showing aortic surface stresses, and (d) front view of one third of the valve showing the ventricular surface stresses

Figure 82. Images showing the coaptation interaction during diastole for a (a) BARD-reinforced leaflet mounted on a $48.5 \%$ styrene stent and a (b) bioprosthetic valve adapted from Labrosse et al. [65].

Figure 83. Dip-coated JSI-reinforced, high profile valve showing hole formation at the mid-valve region of cycling equivalent to 6.12 years

Figure 84. Critical areas of interest when comparing the leaflet stress concentrations, where (1) is the top of the commissure, (2) is the lower commissure, (3) is the center of coaptation, and (4) is the belly. For the higher profile valves, a fifth region defined as the mid valve was added between regions 3 and 4 .

Figure 85. Peak stress concentrations for regions $1-4$ of all models at the (a) aortic and (b) ventricular surface.

Figure 86. Peak stress concentrations for regions $1-4$ for all BARD valve models at the (a) aortic and (b) ventricular surface.

Figure 87. Peak stress concentrations for regions $1-4$ for all valve models including a $48.5 \%$ styrene at the (a) aortic and (b) ventricular surface.

Figure 88. Peak stress concentrations for regions $1-4$ for all JSI-reinforced valve models at the (a) aortic and (b) ventricular surface.

Figure 89. Peak stress concentrations for the aortic surface of all valves at the top of the commissure.

Figure 90. Peak stress concentrations for the aortic surface of all valves at the lower commissure.

Figure 91. Peak stress concentrations for the aortic surface of all valves at the center of coaptation.

Figure 92. Peak stress concentrations for the aortic surface of all valves in the belly region.

Figure 93. Peak stress concentrations for the high profile JSI-reinforced valves at the mid-valve region. 
Figure 94. Isotropic leaflet with mechanical property mimicking that of higher modulus JSI-reinforced orientation $(\mathrm{E}=14.5 \mathrm{MPa})$ with slight coaptation curvature mounted on a $30 \%$ styrene, high profile stent (IsoHi-HiP). Views include (a) top view of aortic surface stresses, (b) top view of ventricular surface stresses, (c) front view of one third of the valve showing aortic surface stresses, and (d) front view of one third of the valve showing the ventricular surface stresses.

Figure 95. Isotropic leaflet with mechanical property mimicking that of lower modulus JSI-reinforced orientation $(\mathrm{E}=2.97 \mathrm{MPa})$ with slight coaptation curvature mounted on a $30 \%$ styrene, high profile stent (IsoLo-HiP). Views include (a) top view of aortic surface stresses, (b) top view of ventricular surface stresses, (c) front view of one third of the valve showing aortic surface stresses, and (d) front view of one third of the valve showing the ventricular surface stresses.

Figure 96. Orthotropic leaflet with mechanical property mimicking that the natural valve $\left(\mathrm{E}_{\text {circ }}=5.79 \mathrm{MPa}, \mathrm{E}_{\mathrm{rad}}=0.966 \mathrm{MPa}\right)$ with slight coaptation curvature mounted on a $30 \%$ styrene, high profile stent (Natural-HiP). Views include (a) top view of aortic surface stresses, (b) top view of ventricular surface stresses, (c) front view of one third of the valve showing aortic surface stresses, and (d) front view of one third of the valve showing the ventricular surface stresses.

Figure 97. Peak stress concentrations for regions $1-4$ on the aortic surface for all JSI-reinforced valve models and the high profile models including the orthotropic natural valve properties and the isotropic $(\mathrm{E}=2.97 \mathrm{MPa}$ and $\mathrm{E}=14.5$ $\mathrm{MPa}$ ) properties.

Figure 98. Component stress concentrations in the circumferential (S11) and radial (S22) directions for all models at the top of commissure.

Figure 99. Component stress concentrations in the circumferential (S11) and radial (S22) directions for all models at the center of coaptation.

Figure 100. Component stress concentrations in the circumferential (S11) and radial (S22) directions for all models at the belly.

Figure 101. Cyclic tension-tension fatigue results for solvent-cast BARD reinforced leaflet samples along the circumferential, radial, and $45^{\circ}$ between the circumferential and radial directions. Each data set was fitted to a log-linear curve whose equation is provided in the figure.

Figure 102. Representation of the principal directions subjected to tensiontension fatigue. The stresses along the $45^{\circ}$ orientation were broken up into their circumferential and radial components. 
Figure 103. Representation of the method used to define a failure envelope for leaflet fatigue at 1 million cycles.

Figure 104. BARD-reinforced leaflet model mounted on a $48.5 \%$ styrene, medium profile stent. Images show the circumferential $\left(\sigma_{11}\right)$ and radial $\left(\sigma_{11}\right)$ stress components at 0.0298 seconds, 0.0571 seconds, and 0.090 seconds during the quasi-static diastolic loading. Note: stress shown in MPa.

Figure 105. Failure envelopes predicted for the BARD-reinforced leaflets, showing the 1 million, 10 million, and 350 million cycle failure envelope. The model-predicted stress components for the top of the commissure, the lower commissure, the center of coaptation, and the belly region are plotted for 0.0298 seconds, 0.0571 seconds, and 0.090 seconds for assessment of fatigue life.

Figure 106. Dimensioned drawings of low profile, high profile, and medium profile stents.

Figure 107. Photogrammetry image pairs corresponding to Frame 1 in Figure 66........210

Figure 108. MatLab regeneration of fiducial points from the $\mathrm{x}, \mathrm{y}$, and $\mathrm{z}$ coordinated generated in Photomodeler ${ }^{\circledR}$ from Figure 107.

Figure 109. Photogrammetry image pairs corresponding to Frame 2 in Figure 66.......210

Figure 110. MatLab regeneration of fiducial points from the $\mathrm{x}, \mathrm{y}$, and $\mathrm{z}$ coordinated generated in Photomodeler ${ }^{\circledR}$ from Figure 109.

Figure 111. Photogrammetry image pairs corresponding to Frame 3 in Figure 66........211

Figure 112. MatLab regeneration of fiducial points from the $\mathrm{x}, \mathrm{y}$, and $\mathrm{z}$ coordinated generated in Photomodeler ${ }^{\circledR}$ from Figure 111.

Figure 113. Photogrammetry image pairs corresponding to Frame 4 in Figure 66........212

Figure 114. MatLab regeneration of fiducial points from the $\mathrm{x}, \mathrm{y}$, and $\mathrm{z}$ coordinated generated in Photomodeler ${ }^{\circledR}$ from Figure 113.

Figure 115. Photogrammetry image pairs corresponding to Frame 6 in Figure 66.......212

Figure 116. MatLab regeneration of fiducial points from the $\mathrm{x}, \mathrm{y}$, and $\mathrm{z}$ coordinated generated in Photomodeler ${ }^{\circledR}$ from Figure 115.

Figure 117. Photogrammetry image pairs corresponding to Frame 8 in Figure 66........213

Figure 118. MatLab regeneration of fiducial points from the $\mathrm{x}, \mathrm{y}$, and $\mathrm{z}$ coordinated generated in Photomodeler ${ }^{\circledR}$ from Figure 117. 


\section{INTRODUCTION}

\subsection{General Statement of Problem Area}

Diseased and dysfunctional heart valves are routinely repaired or replaced through surgical intervention. If damage is too severe to enable valve repair, the native valve is replaced by a prosthetic valve. According to the 2006 American Heart Association Statistical Update [1], an estimated 95,000 inpatient valve procedures were performed in the United States alone during 2003, with the majority of the patients over the age of 65 . The average cost per procedure was $\$ 118,656$ and the in-hospital death rate was 5.6\%.

Commercially available prosthetic valves are either mechanical or bioprosthetic in nature. The designs of mechanical valves available today include the tilting disc and the bileaflet valve; while the bioprosthetic valves consist of either a homograft or heterograft, whose source is a porcine aortic valve or bovine pericardium. There is not a vast array of valve prostheses available for implant, with the majority representing variations of successful designs that have been in existence for the past $35-45$ years [2]. Only minor improvements have been made to these valves over the years; yet they continue to exhibit problems, including thromboebolism in mechanical valves and reduced durability in bioprosthetic valves $[2,3]$. Patients receiving mechanical valves are subjected to lifelong anticoagulant therapy, which is not required for the bioprosthetic valves that produce physiological hemodynamics.

Polymer trileaflet valves have been investigated in an attempt to produce a valve alternative that is both durable and non-thrombogenic. Human implantation of flexible polymeric aortic valve prostheses was carried out in the late 1950's, but persistent problems with these valves included thromboembolic complications and overall valve 
degeneration [4]. The success of mechanical valves resulted in the withdrawal from clinical use of these polymeric valves, although further research and analyses in this area continued in parallel with the mechanical and bioprosthetic valves. To date, polymeric valves have met with limited success due to calcification, thrombogenicity, and long-term material degradation as a result of oxidative reactions and high dynamic stresses borne by the material [4-6]. Polymers utilized included silicone rubber, Teflon $\AA$, polyetherurethaneurea, polycarbonate-urethane, and most commonly, segmented polyurethanes. By selecting an oxidatively stable and durable polymer in combination with refining valve design, it is proposed that a polymer trileaflet valve can be developed that has equivalent, if not superior, durability than mechanical valves and that does not require the use of anticoagulant therapy due to its physiological hemodynamics.

With this objective in mind, a novel polyolefin, poly(styrene-b-isobutylene-bstyrene) (also known as polyștyrene-polyinsobutylene-polystyrene, or "SIBS"), was selected for the design of a fiber-reinforced polymer trileaflet heart valve [7]. SIBS has been proven to be stable in oxidative and acidic environments through a combination of degradation resistance and in vivo transplantation [8]; it has been proven to be equally as thromboresistant as polyurethane by measuring platelet deposition with radiolabelled platelets in a parallel plate flow configuration [7]; and the fatigue resistance of fiberreinforced SIBS has been proven through a combination of cyclic tensile and bending fatigue tests [7]. The numerous different iterations of valve designs that have been evaluated include: different reinforcement techniques, variable valve geometries, varying stent flexibilities, and variable SIBS formulations [9-14]. The hydrodynamics of each of 
these iterations has been encouraging, but some of these valves failed prematurely during in vivo implantation in a sheep model or during accelerated in vitro fatigue.

On average the heart contracts 70 times per minute, which equates to 100,000 cycles per day, 35 million cycles per year, or 2.5 billion cycles per lifetime. The natural valves are complex, anisotropic structures with the innate ability to sustain the alternating flexural fatigue without impairment; furthermore, they are endowed with the capacity to self-repair [3], putting them at an advantage over the static mechanical, bioprosthetic, and polymer trileaflet valves. Nevertheless, if it were possible to elucidate what primary characteristics provide the natural valve with its fatigue resistance, it is hypothesized that one could produce a polymer trileaflet valve with improved endurance.

\subsection{Research Purpose}

The long-term objective of this research is to develop a polymer trileaflet valve with superior hemodynamics, durability, and biocompatibility than both commercially available mechanical and bioprosthetic valves. If this goal is realized, the valve has the potential to be an alternative for aortic valve replacement.

The more immediate goal, and the focus of this work, was to assess the design parameters that had the greatest impact on both valve function/hemodynamics and durability. Knowledge of the natural valve, and bioprosthetic valve experience, was used to identify the particular characteristics that have the greatest influence on valve hemodynamics and durability for incorporation into the polymer valve design. Aspects of the valve that were analyzed included: (1) leaflet geometry, (2) support structure (stent) and mechanics, and (3) fiber reinforcement. A finite element model was 
developed to determine the impact of each of these critical parameters on leaflet stress distribution, and this information was employed during experimental evaluations of the valve and each of its components, thereby verifying the impact of different valve designs on hemodynamic and fatigue performance.

The final outcome of this work was a mathematical model used to predict fatigue in the valve. Experimental assessment of valve durability is a time-consuming process, where 15 years (600 million cycles) worth of fatigue data takes approximately one year to collect. The fatigue model can be incorporated as a design tool, accelerating the development process by eliminating valves with insufficient durability. 


\section{BACKGROUND AND SIGNIFICANCE}

\subsection{The Heart \& Valve Disorders}

The heart is a muscular organ whose function is to deliver blood to the rest of the body [15]. It is separated into a left and right side, each composed of atrial and ventricular compartments. During diastole, both atrioventricular valves (tricuspid on the right and mitral on the left) are open, allowing the ventricles to fill with blood. During systole, the ventricles contract in unison, pumping blood into the pulmonary and systemic circulations. Blood enters the right hand side of the heart through the vena cava, and is routed through the atrium and ventricle before being pumped into the pulmonary circulation and lungs. Oxygen-rich blood returns to the left atrium and then ventricle, where it is pumped into the systemic circulation.

Valves are located at the exit of each compartment to control the unidirectional flow of blood through the cardiovascular system. They open and close in response to pressure differentials as the heart contracts and relaxes [15]. The mitral and aortic valves, located on the left hand side of the heart, are the most common sites for heart valve disease as a result of the left heart's significantly higher workload [16]. Valvular heart disease can be as a result of either congenital or acquired defects, including rheumatic fever, endocarditis, calcific degeneration, myxomatous degeneration, or congenital anomalies [16]. The two main problems associated with valvular disease are stenosis and insufficiency/regurgitation. In the former case, the opening through which blood can pass becomes narrowed due to the leaflets either becoming rigid or fused together. In the latter case, the valve does not close completely, and a portion of the ejected blood flows backwards. As a result of both, blood accumulates in the chamber, and the heart is 
required to work harder in order to supply the body. This increased workload leads to the heart muscle thickening and dilating and can result in congestive heart failure. Once the heart valve has lost its normal functioning ability, drugs can only relieve the symptoms. Valve replacement surgery is recommended when damage to the valve is considered to be significant enough to be life threatening.

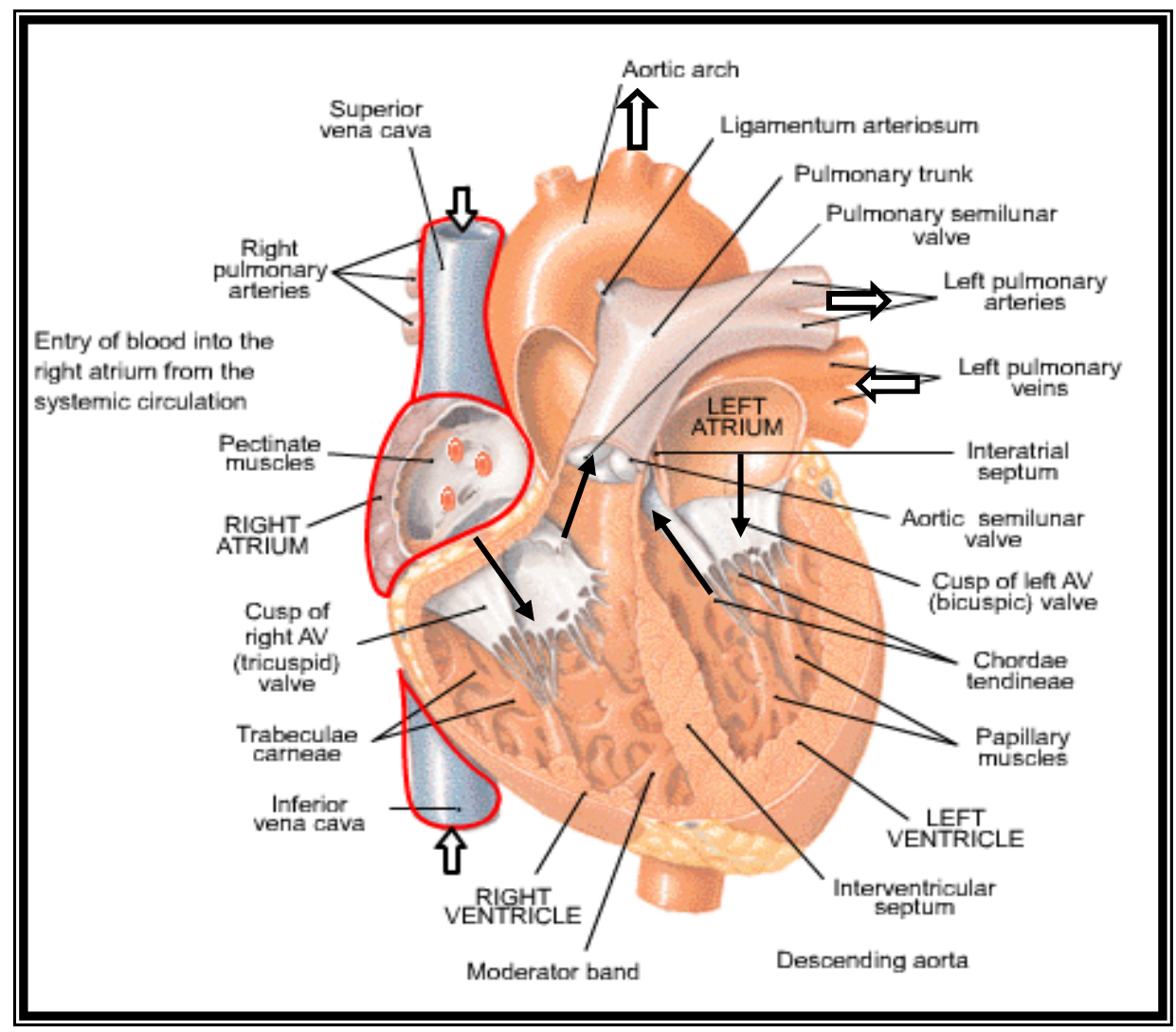

Figure 1. A diagrammatic frontal section through the heart, showing major landmarks and the path of blood flow [17].

\subsection{Commercially Available Prosthetic Valves}

The complete replacement of damaged and diseased heart valves by prostheses has become routine practice, but the question remains, which valve prosthesis should be chosen? Commercially available valves can be divided into two primary classes, 
mechanical and bioprosthetic, each with its associated advantages and disadvantages. Factors influencing which valve is most suited to a patient include: the patient's age, comorbidities, need for associated procedures, availability of a given replacement, patient agreement, and surgeon expertise [2]. Present regulations for heart valves are very strict, making it difficult for new valve designs to enter the market place. As a result, the valves available today represent variations of prostheses with a long-proven history $[2,18]$.

\subsubsection{Mechanical Valves}

Mechanical valves, that are well-known for their durability, are the preferred valve for individuals under the age of 65 [2]. There was an assortment of mechanical valves available in the past, but the primary designs implanted today include the tilting disc design that was introduced in 1969 (Figure 2b), the bileaflet design that was available from 1977 (Figure 2c), and to a lesser extent, the ball and cage design that was developed in the 1960's (Figure 2a) [3, 18, 19]; the former two valves utilized pyrolytic carbon.

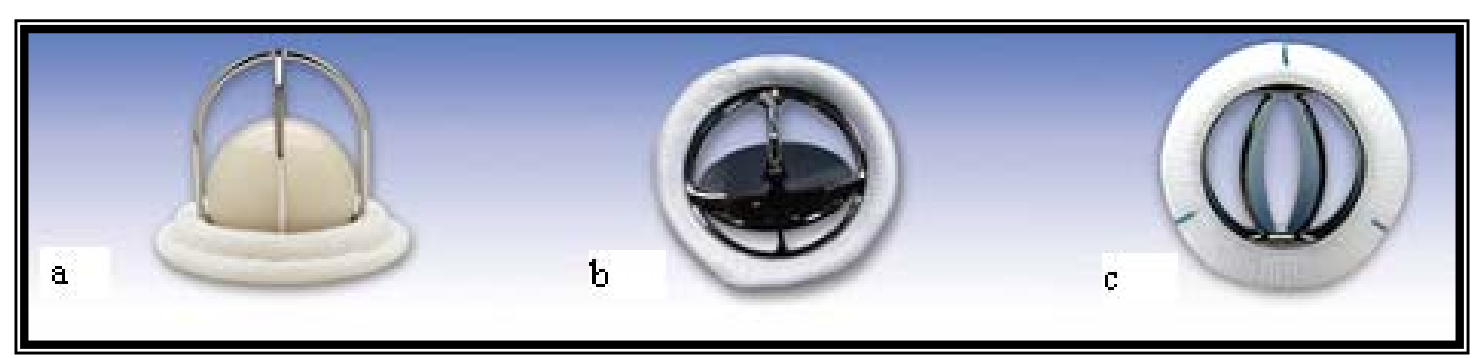

Figure 2. Three basic types of mechanical heart valves: (a) Ball and cage valve, (b) Tilting disk valve, and (c) Bileaflet valve (http://www.edwards.com/PatientsandFamilies/MyHeart/ClinicalProcedures/ ValveReplacementEU.aspx).

According to a report by Senthilnathan et al. [18], the St. Jude and Carbomedics bileaflet mechanical valves were the most widely implanted valves in the United Kingdom in 1996 , accounting for $41 \%$ of the valves implanted. The advantages of the 
bileaflet mechanical valves include that they have a low profile so can be implanted into smaller hearts without obstructing any components, such as the mitral valve and coronaries; they have good hemodynamics in that the transvalvular pressure gradient is low with minimal regurgitation; and they are durable, showing a low occurrence of mechanical failure $[2,3,18]$. The tilting disc valves, including the monostrut BjorkShiley and Medtronic Hall, were the second most commonly implanted mechanical valves, accounting for $7 \%$ of the valves implanted. Like the bileaflet valves, the tilting disc valves have a proven history of durability. The third and least commonly implanted valve on this list is also the oldest valve: the Starr-Edwards Caged Ball valve. While not having as favorable hemodynamics as the bileaflet and tilting disc valves, it is still a reliable valve that is used when surgeons require a valve that is easy to handle under difficult surgical circumstances $[18,19]$. In a more recent study by the 2002 , Health Research International [20], it was reported that mechanical valves had lost a portion of the market share to the bioprosthetic valves, whose further development has increased their expected durability. In the 2002 report, mechanical valves only accounted for $40 \%$ of the market, with the St. Jude bileaflet valves still being the most popular.

One common problem for all the mechanical valves is that their design results in partial occlusion of blood flow, leading to non-physiological flow characteristics $[2,3$, 18]. It is this characteristic that contributes to morbidity and mortality as a result of thrombosis, embolism, and bleeding complications. Consequently, patients receiving mechanical valves are subjected to life-long anticoagulation therapy. The problems associated with the long-term use of warfarin include insufficient monitoring, resulting in either under or over anticoagulation, and hemorrhagic complications. 


\subsubsection{Bioprosthetic Valves}

Since their introduction into the market in the 1970's, bioprosthetic heart valves have been plagued with problems resulting in decreased durability when compared with mechanical valves $[2,3,18,21]$. They do, however, reproduce the central flow characteristics of the natural valve and are less thrombogenic than mechanical valves; therefore, long-term anticoagulation treatment is not required. As a result, bioprosthetic valves are chosen for older patients with a life expectancy less than 10 - 15 years and for younger patients in whom anticoagulation therapy is contraindicated.

There are three tissue sources utilized in bioprosthetic valves: human, glutaraldehyde-treated porcine aortic valves (Figure $3 \mathrm{a}$ and Figure 3c), and glutaraldehyde-treated bovine pericardium (Figure 3b). The homografts, which are human valves excised from cadavers, are the least commonly used due to an overall shortage in both numbers and sizes and because they are difficult to insert $[2,18]$. The stented porcine (Figure 3a) and bovine (Figure 3b) valves are the most commonly implanted, with Medtronic and Edwards Lifesciences being the dominant suppliers [2]. According to the 2002, Health Research International report [20], Edwards Lifesciences accounted for $74 \%$ of stented valve units and sales in 2001 , with the Carpentier-Edwards Perimount pericardial prosthesis being the most popular. Medtronic accounted for almost $26 \%$ of sales, mainly due to their experience with the Hancock porcine bioprosthesis. Both valves are said to be third-generation valves with an approximate durability of 1015 years [2].

The stented valves, either porcine or bovine, are supported by a metallic or polymer structure/stent. The stent allows for ease of implantation, but the downfall is 
that it results in a stenotic region caused by partial orifice occlusion [2]. This prompted the development of stentless porcine valves (Figure 3c), which consist of aortic roots modified with a sewing ring $[2,18]$. The entire root is implanted either within the native root or in place of the native root, and the design provides the advantage of an increased orifice area. This design is significantly more complicated to implant than the stented version, and conclusive information as to the long-term durability is still unknown.

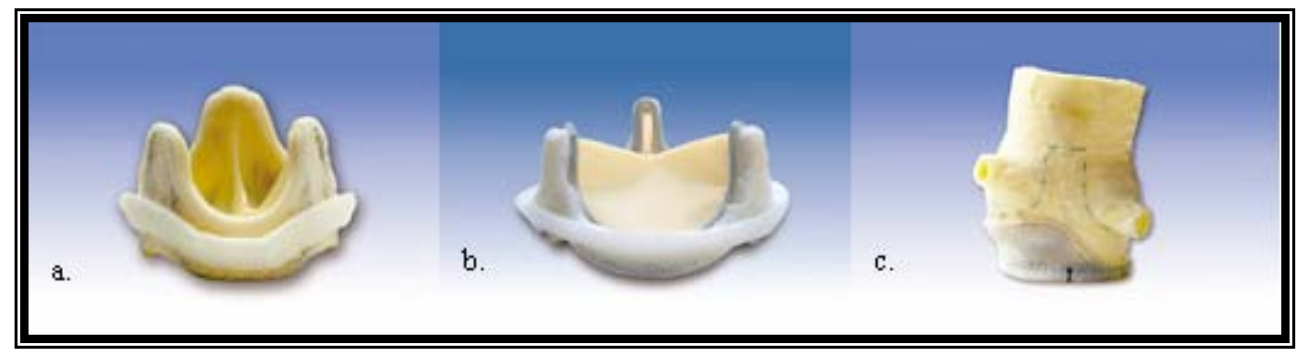

Figure 3. Three types of bioprosthetic heart valves: (a) Stented porcine valve, (b) Stented bovine pericardial valve, (c) Stentless porcine valve (http://www.edwards.com/PatientsandFamilies /MyHeart/ClinicalProcedures/ValveReplacementEU.aspx).

When comparing stented pericardial versus porcine valves, pericardial valves can be fabricated into a multitude of designs, whereas porcine valve designs are restricted by the valve anatomy. Pericardial valves are fabricated from flat sheets of glutaraldehydefixed bovine pericardium that are oriented to mimic the natural/porcine valve in both form and function [18]. The pericardial valves tend to have superior hemodynamics to the porcine valves as a result of their improved inner-to-outer diameter ratio and leaflet dynamics during forward flow; however, the traditional designs have been modeled to exhibit significantly higher stresses when loaded in tension (i.e. during diastole).

The primary problem with all xenogenic prostheses is tissue failure, which often begins within 10 years of implantation [2, 3, 18, 21]. Degradation is as a result of 
calcification, mechanical damage, or a combination of both, and it has been linked to glutaraldehyde fixation and the stent-valve interaction, among other things. Glutaraldehyde treatment effectively cross-links the tissue and reduces its antigenicity while preventing proteolytic degradation. As a consequence, the tissue loses its mechanical compliance, and the resultant increase in leaflet stress concentrations accelerates fatigue. The dead, phosphate-rich cells are proposed to initiate calcification: when they come into contact with calcium-rich plasma, the calcium accumulates and forms calcium phosphate [21]. The presence of calcium deposits on the leaflets can result in stenosis and leaflet tearing.

The world market for bioprosthetic heart valves has increased by approximately $5 \%$ per year [21]. This is due in part to the increasing percentage of individuals over 65 but also as a result of developments that have increased bioprosthetic valve durability.

\subsection{Polymer Trileaflet Valves}

Decades of research have gone into the development of the two commercially available valve categories. Although these valves have met with clinical success, the mechanical valves still require anticoagulant therapy and the bioprosthetic valves lack durability. In theory, the flexible membrane polymer trileaflet valve design was meant to overcome the disadvantages of these commercially available valves and provide a valve alternative that is both durable and non-thrombogenic. In reality, this has not been the case; a successful polymer valve for human implantation is not yet available. Failure of polymer valves has been caused by calcification, thrombogenicity, and long-term material degradation as a result of oxidative reactions and high dynamic stresses borne by 
the material $[4-5,22-26]$. A combination of inappropriate design and material selection has been blamed.

Flexible polymer heart valve prostheses are not a new concept. Roe began human implantation of a flexible silicone rubber valve in 1958, but mortality and morbidity due to embolization resulted in cessation of the study $[4,26]$. Shortly after that, Braunwald implanted a tricuspid polytetrafluoroethylene (PTFE) valve in the aortic position of 23 patients. Mortality was again high in these patients as a result of thickening and rupture of the valve leaflets. Two more clinical studies performed by Roe in 1966 and Hufnagel in 1977 had similar clinical outcomes with high patient mortality. The 1966 study included a Dacron and silicone prosthesis, and the 1977 study utilized a monoleaflet Dacron prosthesis.

Due to the success of the mechanical and bioprosthetic valves, human implantation of polymeric valves stopped in the $1980 \mathrm{~s}$, although research continued. Animal testing of a range of different polymers and designs continued to have problems related to valve durability. Materials used and tested in these valves included silicone rubber, Silastic ${ }^{\circledR}$, PTFE, segmented polyurethanes, and Biomer ${ }^{\circledR}$ (a segmented aromatic diamine chain extended polyetherurethane urea (PEUU)) $[4,5,26]$, and failure modes included thrombosis, calcification, oxidation, hydrolysis, and mechanical breakdown occurring either alone or in conjunction with the previous mechanisms.

The trileaflet polyurethane design is the most highly investigated valve to date. The trileaflet valve design allows the valve to mimic the hemodynamics of the natural valve, while the implementation of a block polymer makes it possible to control the physical, mechanical, and chemical properties of the valve [22]. Bernacca, Mackay, 
Wheatley, and colleagues have concentrated their efforts on polyurethane for trileaflet heart valves, but their results revealed that calcification, lack of durability, and thrombus formation were still existent problems preventing the success of these valves $[4,22,23$, $24,26]$. In addition to these findings, biodegradation of polyurethanes in vivo has been a persistent problem $[24,27]$. Polyurethanes have proven to have suitable mechanical strength, flexibility, and durability for use in trileaflet heart valves, which led Bernacca et al. [24] to adopt a biostable polyurethane with a silicone-based soft segment chemistry. The two particular polymers chosen included EV3.35 which incorporated a diol chain extender and EV3.34 which incorporated a diamine chain extender. These polyurethanes have shown resistance to biodegradation, and after implantation for 6 and 9 months in the mitral position of 18 month old sheep, no incidence of thrombus formation, calcification, fibrin deposition, and mechanical breakdown was evident [24]. Young adult sheep are not a good model for calcification and sheep in general have a lower incidence of thrombosis than humans, so conclusive evidence as to the potential of this valve is still elusive $[24,26]$.

Valve durability is not only a function of the polymer selected for leaflet construction, it is highly dependent on valve design and manufacturing process. As was evident in many polyurethane prostheses, calcium accumulates in regions of high stress concentrations and surface defects, so mechanical degradation can act as a precursor to calcification [23]. It is hypothesized that by closely mimicking the natural valve's transvalvular flow pattern, one can produce a valve with increased durability and reduced incidence of thrombosis. It was with this concept in mind that Daebritz et al. $[4,26]$ developed mitral and aortic polycarbonate-urethane (PCU) prostheses. The aortic PCU 
prosthesis adopted a leaflet configuration and thickness distribution that effectively reduced the stresses and strains in the leaflet for increased durability. The design also included a supporting stent and sewing cuff that were minimized to increase the effective orifice area, thereby decreasing transvalvular pressure drop. In vivo evaluation of these valves was carried out in a growing calf model, where incidence of calcification and thrombosis are generally high. The PCU aortic valves were implanted with a Medtronic Mosaic ${ }^{\circledR}$ and an Edwards Perimount bioprosthesis as controls. The animals with the bioprostheses died prematurely due to severe valve degeneration and stenosis, whereas five of the seven animals with the PCU valve survived the full 20 week study. The two animals that died prematurely were as a result of subvalvular pannus overgrowth, and the remaining valves were found to have minimal incidence of degeneration with calcification. The calcific deposits were only present on the polymer surface. In vitro accelerated fatigue of these valves is ongoing, and an equivalent of 7.9 years of cycling has been attained without failure. Thus far, this appears to be the most successful polymer trileaflet valve to date, but further information as to the valve's in vitro and in vivo function is required.

The high tensile and bending stresses borne by the leaflet material can lead to the valve's ultimate failure. Reduction of stress concentrations have been attempted by changing leaflet geometry, manufacturing techniques, and frame mounting methods, but according to Cacciola et al. and De Hart et al. [28-30], fiber reinforcement may be the answer. Due to the fact that natural valve leaflets are a composite design with stressreducing collagen fibers, it has been proposed that the incorporation of fiber reinforcement in polymeric trileaflet heart valves can reduce the stress concentrations in 
the matrix, potentially increasing valve durability due to a reduction in tears and perforations. De Hart et al. [29] performed a three-dimensional analysis of a stented aortic valve with either a circumferential or sinusoidal fiber reinforcement. A decrease in stress of up to $60 \%$ was observed, where the load was transferred to the higher-modulus fibers. Cacciola et al. [30] repeated this study for a stentless aortic valve, where a 75\% reduction in stress was achieved. The stress-reducing capabilities of fiber reinforcement are obvious from these studies, even though non-physiological reinforcement geometry was incorporated. By adopting a more physiological fiber layout, it is assumed that a further reduction in stress concentration can be achieved, creating a valve leaflet with unrestricted motion during opening and closing and strength and stiffness while the valve is closed.

\subsection{Design Criteria: The Natural Aortic Valve as a Model}

The natural valve is a complex anisotropic structure that is able to endure 2.5 billion cycles in a lifetime without generating regurgitation, hemolysis, thrombosis, or extreme stress concentrations in the leaflets or surrounding tissue $[3,4,26]$. No other prosthetic valve has been capable of replicating the natural valve's performance or durability, but the secret to their success may lie in the form and function of the natural valve. Synthetic polymer trileaflet valves have the greatest potential to replicate the critical properties of the natural valves as, unlike bioprosthetic valves, they have no constraints with respect to geometry and mechanical properties. One can hypothetically produce a synthetic valve that more closely approximates the natural valve than any prosthetic valves have to date. What is known about the natural valve and what has been 
learned from bioprosthetic valves can be applied to polymer trileaflet designs for improvement of valve function and durability. The potential for a polymer valve's

success is supported by reports by Daebritz et al. [4, 26], whose close approximation of the natural valve's flow characteristics produced a valve with a low incidence of thrombosis in vivo, and Cacciola et al. [28, 30] and de Hart et al. [29], whose use of fiber reinforcement resulted in a decrease in leaflet stress concentrations during diastolic loading.

The valve leaflet has a specialized structure that allows it to endure large tensile stresses during diastole, while allowing wide rotations during systole. In the following section, each of the valve's predominant tissue elements and primary structural organizing centers will be discussed in relation to the role they play in valvular mechanics. In addition, the properties of bioprosthetic valves and their deviation from the natural valve will be discussed with respect to their impact on function and durability.

\subsubsection{Tissue constituents}

Historically, the natural aortic valve has been viewed as a tri-layered structure consisting of a thin ventricularis layer, a thicker fibrosa layer, and an inner spongiosa layer (Figure 4) that are free to move with respect to one other as the valve opens and closes [3, 31-35]. Histological analysis of thin tissue sections has revealed that the ventricularis layer is composed of a network of collagen and elastin fibers, the fibrosa layer consists primarily of collagen bundles that are believed to be the primary stressbearing elements in the leaflet, and the spongiosa layer is a proteoglycan-rich layer that separates the ventricularis and fibrosa. Under resting conditions, the fibrosa and ventricularis are preloaded, with the fibrosa under compression and the ventricularis 
under tension [35]. The surface is covered with a layer of endothelial cells providing additional thromboresistance. Each of the connective tissue elements of the valve are found in different quantities and orientations throughout the leaflets and supporting structures, and their construction is directly related to the role they play in valve mechanics [36].

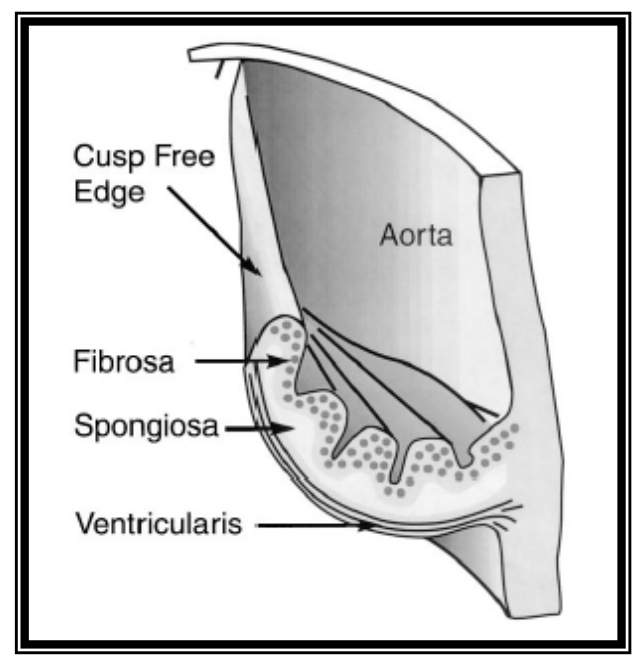

Figure 4. A cutaway through the leaflet and aortic wall showing the internal configuration of the fibrosa, spongiosa, and ventricularis. Adapted from Vesely [32]

\subsubsection{1. $\quad \underline{\text { Collagen }}$}

Collagen fibers and fiber bundles are believed to control the shape of many of the valve components, including the fibrous ring, commissures, and leaflets [36]. It is this particular tissue element that is said to endow the natural aortic valve with its high fatigue strength. Thick collagen fibers are found beneath the endothelium of the aortic valve and are interspersed with elastin fibers and thin collagen fiber bundles. The thick collagen fibers emanate from the commissures in a circumferential direction, forming the leaflet belly and free edge (Figure 5) [31,36]. Thin collagen fibers are interwoven with the thick bundles and form a network that radiates and branches in multiple directions while 
maintaining the collagen cross sectional area. The density of these fibers is proportional to the local leaflet load. In their relaxed form, collagen fibers display crimped patterns, but when they are loaded in tension, the collagen straightens and assumes the load.

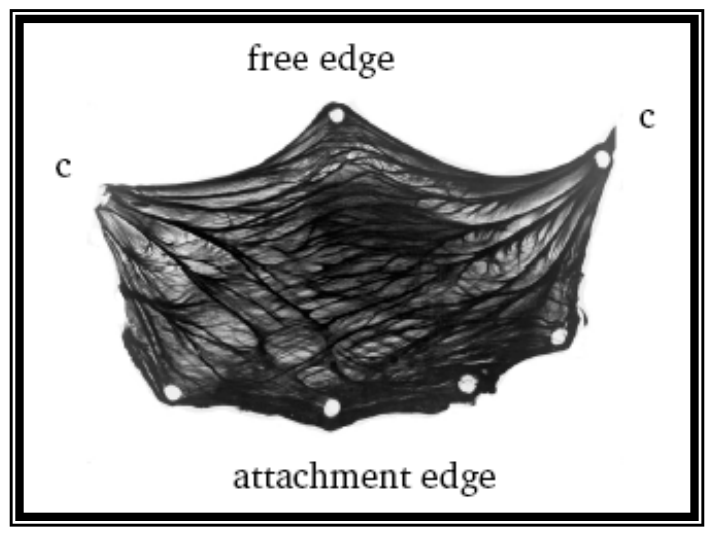

Figure 5. Typical collagen fiber architecture in the aortic valve leaflet [37]. The commissures are denoted by 'c'.

Doehring et al. [31] utilized a system that combined high resolution digital imaging with transmitted elliptically polarized light to visualize the collagen structures in porcine aortic valve leaflets. Instead of finding the collagen predominantly in the fibrosa layer, as was the historical view, the collagen fibers were found to be in a more complex branching network in multiple layered membrane mesostructures. This multi-layered arrangement was proposed to facilitate valvular function by allowing the layers to slide upon each other during valve cycling, thereby allowing leaflet flexibility without sacrificing the tensile strength provided by the collagen fibers. In addition to this, Doehring et al. concluded that, although the general collagen architecture was in the form shown in Figure 5, each of the three cusps was not identical: the non-coronary cusps typically had narrow fiber bundles that were long and did not branch extensively; the right and left cusps had fewer large bundles and extensive branching; the non-coronary 
and left coronary cusps appeared to be mirror images of each other, and the right coronary cusp was self symmetric. The development of collagen architecture results from cuspal loads, so it may be possible that non-uniform loading created this nonuniform distribution from one leaflet to another. The cusps' sizes were also found to be different, where the non-coronary cusp was significantly smaller than the right and left cusps. These factors could have implications in surgical orientation of porcine valves and the symmetry of bovine pericardial and polymer trileaflet valves.

Collagen fibers are thought to be the predominant valve constituent when considering tensile properties; however, the fiber interaction and orientation does play a role in the flexural properties of valve leaflets $[38,39]$. The impact of collagen fiber reinforcement during systole was evaluated in a numerical study performed by de Hart et al. [38]. The numerical model implemented the natural collagen reinforcement pattern in a three-dimensional model with a fully coupled fluid-structure interaction. The values applied for the Reynolds and Strouhal number were below the characteristic physiological values due to model constraints, but a comparative analysis with a nonreinforced model revealed that the presence of fiber reinforcement reduced the peak systolic matrix stress by $63 \%$ while stabilizing leaflet motion during systole.

In the natural valve, the collagen orientation is not anticipated to impact the transvalvular pressure drop, but the combined tensile and compressive properties of the leaflet as a whole do contribute to its flexural stiffness. In order to improve hemodynamics one must reduce the flexural rigidity of the valve leaflets. Bioprosthetic valves made from bovine pericardium do not have the tri-layered structure that native human and porcine valves do, and yet they are capable of maintaining an equivalent 
durability to the porcine valves. Bovine pericardium lacks the spongiosa layer that is rich in GAGs, and the impact of this was seen by Mirnajafi et al. [39] in a 3-point bending evaluation of bovine pericardium and porcine leaflet specimens. The curvature versus applied moment response of the porcine tissue was a linear one, whereas the bovine pericardial response was non-linear and dependent on collagen fiber orientation. The linear response of the porcine tissue was attributed to the GAG content that allowed the collagen layers to slide with respect to one another. Bovine pericardial tissue showed increased stiffness when it was flexed perpendicular to the collagen fiber orientation, so pericardial orientation can have an impact on valvular mechanics. In the same study, glutaraldehyde fixation was found to increase the flexural stiffness of bovine pericardial tissue. This fixation method results in inter- and intramolecular crosslinks within collagen fibers, so non-collagenous tissue components and collagen interactions/crosslinks are believed to play the major role in flexural stiffness, not the stiffness of the collagen fibers themselves. The important aspects of this study that can be applied to synthetic valves are: (1) exact replication of the natural collagen architecture is not essential for valve durability, (2) fiber orientation can affect the flexural stiffness of leaflets and (3) free movement of fibers with respect to one other can enhance the flexural properties of leaflets, while inter-fiber linkage can lead to matrix stiffening.

\subsubsection{Glycosaminoglycans (GAGs)}

GAGs are the ground substance for collagen and elastin fibers and are found within the interfibrillar spaces and linked to collagen. They are believed to provide the 
native cusp with its three-dimensional architecture while creating an environment that influences the mechanical behavior of the valve $[33,36]$.

The three primary GAGs found in porcine aortic valves are hyaluronic acid, chondroitin sulfate, and dermatin sulfate [33]. They are negatively charged, hydrophilic molecules that have the potential to absorb high quantities of water and, therefore, hydrate the extracellular matrix. GAGs are a large constituent of the extracellular matrix of porcine valves, especially within the spongiosa, whose primary function is to allow shearing between the collagen fibers of the fibrosa and ventricularis layer. It is this function that results in a reduction of stresses related to leaflet flexure and allows the collagen fibers to move with respect to one other without resulting in wear-related fatigue. Additional functions of GAG molecules are their ability to sustain compressive forces and diminish calcification by chelating calcium ions and preventing hydroxyapetite nucleation.

Glutaraldehyde fixation and storage of bioprosthetic heart valves has been associated with a loss of GAGs. Lovekamp et al. [33] reported that saline storage and/or glutaraldehyde fixation causes an approximate $20 \%$ decrease in tissue thickness with almost a complete loss of GAGs. A $60 \%$ increase in flexural rigidity, associated with the shearing function of GAGs, was reported in GAG-free cusps when compared with controls. This increase in leaflet rigidity has been associated with the premature fatigue of bioprosthetic valves due to cusp delamination and wear.

\subsubsection{Elastin}

Elastin constitutes a small percentage of the aortic valve leaflet's dry weight, accounting for only $13 \%$ (collagen accounts for 50\%) [35]. This low percentage of 
elastin implies that it does not contribute significantly to leaflet mechanics; however, the collagen fibers align and the cusps elongate beyond 50\% strain during diastole and recoil during systole. Since collagen is not a highly elastic material, the high strain and recoil of valve leaflets is believed to be as a result of the elastin components.

Vesely [35] investigated the role of elastin in the fibrosa and ventricularis of porcine aortic valves by comparing the mechanical properties of fibrosa and ventricularis elastin with intact fibrosa, ventricularis, and whole leaflets. Elastin was found to impose tensile loads during valve unloading, and it was believed to enforce the preloaded configuration of the fibrosa and ventricularis. The ventricularis is capable of a $60 \%$ strain in the radial direction with recoil back to its original configuration. Since collagen fibers exhibit low extensibility, they are proposed to be in a corrugated configuration under resting conditions, and application of force results in them straightening until they take up the load and inhibit further extension. The mechanical properties of the leaflet prior to collagen elongation are believed to be dominated by the elastin fibers. Upon release of the load, the collagen fibers spring back to their original configuration as a result of the elastin properties. The ventricularis attains a maximum strain of approximately $20 \%$ in the circumferential direction, where collagen fibers are believed to play a more predominant role in leaflet mechanics. The elastin plays a small part in valve mechanics for the fibrosa layer, where the properties of the wavy collagen dominate; the fibrosa/ventricularis interaction is believed to enable fibrosa restructuring.

Elastic fibers provide the valve with high extensibility in the radial direction of leaflets and in the circumferential direction for the walls of the aortic sinus and ascending 
aorta [36]. The radial extensibility of heart valves promotes leaflet coaptation and reduces regurgitation during diastole.

\subsubsection{Structural Organizing Centers}

Each of the structural organizing centers of the valve are composed of the same basic tissue constituents, but their quantity and arrangement differs dependent on the mechanical loads and function of the organizing centers [36, 40].

\subsubsection{1. $\quad$ Commissures}

The commissure is responsible for supporting large tensile stresses during diastole while allowing large deformations during systole [40]. It is the primary shock absorbing element of the valve leaflet, and it is in this location that the load on the leaflet is effectively transferred to the aortic wall [36].

Collagen fibers in the commissural region are better aligned than in other regions of the valve, such as the belly $[31,36,40]$. At the apex of leaflet attachment, the commissure is composed of thick, twisted bundles of collagen fibers, some of which radiate to form the free edge of the leaflet. It is the twisted makeup of the collagen fibers that provides this region with high tensile strength. Under loaded conditions, such as those during diastole, the fibers rapidly align and straighten, and the extent to which this occurs depends on the amount of ground substance.

Flexure is a key mode of deformation, especially in the commissural area where an approximate $65^{\circ}$ angle of rotation occurs from the fully closed to fully open leaflet configuration [40]. The stiffness in this area has a direct effect on leaflet opening and can have a significant impact on valve hemodynamics; therefore, quantification and design for this flexural stiffness is an important part in prosthetic valve development. According 
to Mirnajafi et al. [40], the flexural stiffness in the commissural region is approximately one third of that in the belly region, and a decrease in flexural stiffness occurs with increasing angle. When flexed in the non-physiological, reverse direction, flexural stiffness increases by approximately $50 \%$, so it can be hypothesized that the valve is constructed for specific unidirectional flexure. The combination of high flexural and tensile stress can accelerate fatigue in this region, thereby making it a critical region for design optimization in bioprosthetic and polymer trileaflet valves.

The commissures are attached to the aortic wall by collagen bands along their entire length. It is through these bands that the load is transferred to the aortic wall [36]. The attachment zone is composed of wavy collagen bands which have a twofold function: they limit the displacement of the commissures, and they shield the leaflet from overloading due to the rapid increase in pressure during diastole. It is likely that the commissure facilitates aortic valve opening through this attachment; the aortic wall dilates during initial systolic ejection, pulling the commissural region as it moves. Early designs of porcine and pericardial valves utilized rigid supporting stents that did not permit commissure dilation during systole [21]. Valve failure was often as a result of tearing at the commissure, and this was linked to the rigid stent. Newer designs incorporated a flexible supporting stent, but no conclusive clinical evidence has been provided as to its benefit. Commissural tears still remain a problem, and the true cause is unknown. Computational modeling has proven the theoretical advantage of flexible stent posts, but further analyses are still required to improve the concept. Two common problems that have been associated with flexible stent posts have been: stent deformation 
during implant and cardiac contraction resulting in valve failure, and polymer stent creep resulting in valve deformation and failure.

\subsubsection{2. $\quad$ Area of Coaptation}

The pressure exerted on aortic valve leaflets during diastole forces them to extend radially and coapt with adjacent leaflets. When the valve is in this closed configuration, the majority of the leaflet is loaded in tension, but the area of coaptation is loaded in a combination of tension and compression [21,31]. It is the radial extensibility of the natural valve that allows the coapting region geometry to be more parabolic in nature, reducing the load on the leaflet. Pericardial valves, especially those that are cross-linked, do not exhibit this radial elasticity, so the design of an appropriate leaflet geometry is required for coaptation. As a result, many pericardial valves have a practically horizontal free edge and spherical leaflet geometry. This deviation from the natural valve geometry has been proven to result in increased stress concentrations in the pericardial leaflet when compared with natural or porcine leaflets [21], but closer replication of the natural valve geometry is not possible due to the lack of distensibility inherent in pericardial tissue.

\subsubsection{3. $\quad$ Fibrous Ring}

The fibrous ring is found at the base of the leaflets where they attach to the aortic wall. This ring was originally believed to be rigid and un-wielding, but animal experiments have shown that a $22-28 \%$ deformation may occur during cardiac ejection [36]. The fibrous ring is composed of circumferentially oriented collagen fibers interspersed with elastin. In the relaxed state, the collagen fibers are slightly rippled, but during systole, circumferential load causes these to straighten, allowing a small increase in circumference. 


\subsubsection{Belly Region}

At the center of the leaflet, or the belly region, the collagen fiber architecture is more disorganized and noticeable spaces exist [36, 40]. Elastin fibers are found between the collagen fibers, and the orientation of each provides the valve with the capacity to undergo large radial deformation while supporting high circumferential stresses. During diastole, when the leaflet is exposed to high tensile loads, collagen fibers undergo gradual alignment when compared with those in the commissural region [40]. There is an increased flexural stiffness in this region, which may facilitate multidirectional flexure without leaflet wrinkling during valve opening.

\subsection{SIBS Polymer Trileaflet Valve}

The SIBS trileaflet valve is being designed with the intention of overcoming the failure modes of previous polymer valves, thereby providing a valve with superior hydrodynamics in addition to increased durability and biocompatibility. Many polymer valve designs have incorporated an isotropic polymer, predominantly polyurethane, and failure modes have been as a result of inappropriate material and design choice. The chemical degradation of polymers in conjunction with material fatigue in vivo can enhance the overall physical degeneration of a valve, thereby accelerating its ultimate failure [7]. Cyclic fatigue of polymers results in the progressive formation of defects that grow and interact, ultimately resulting in the failure of the material at a stress lower than its tensile strength [41]. With the introduction of a fibrous reinforcement, propagation of cracks in the polymer matrix is inhibited by the presence of the fibers, and fatigue of the sample is primarily due to fiber breaks and delaminations [42]. The highest resistance to 
fatigue is achieved when the fibers are parallel to the applied stress. Consequently, a fiber-reinforced polymer trileaflet valve is being designed with an oxidatively stable polymer (SIBS) to reduce chemical degradation and a design reminiscent of the natural valve to reduce material fatigue.

Biodegradation of polymers is due to a cooperative interaction between enzymatic hydrolysis and oxidation [7]. Materials that are more resistant to oxidation are less vulnerable to enzymatic degradation; therefore, overall biodegradation is reduced. Polymer backbones that are composed of alternating quaternary and secondary carbon linkages (including polyisobutylene (PIB)) do not have sites to oxidize, as shown in Figure 6. PIB is not well suited as an elastomer due to its gummy consistency, but by extending the ends of the polymer with harder blocks of polystyrene, a triblock copolymer can be formed with suitable elastomeric properties. With this in mind, a polyolefin, poly(styrene-b-isobutylene-b-styrene) (also known as polystyrenepolyisobutylene-polystyrene, or "SIBS), was chosen for the development of the novel trileaflet heart valve. The polymer's physical properties place it between polyurethane and silicone, and its inertness to oxidative and acidic environments, such as the body, has been proven through a combination of degradation resistance and in vivo transplantation $[7,8]$.

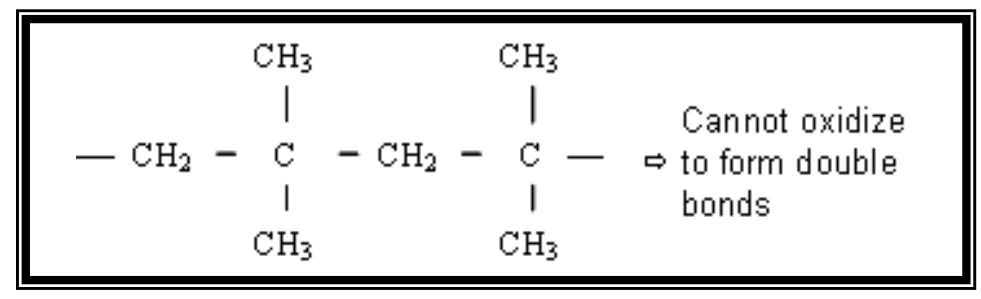

Figure 6. Polyisobutylene backbone showing alternating quaternary and secondary carbon linkages that do not have sites to oxidize. 
Preliminary studies of both the completed SIBS valve and material samples have revealed:

- SIBS valves have comparable hydrodynamics to commercially available mechanical and bioprosthetic valves [9, 10, 43].

- $\quad$ The fatigue life of a SIBS composite is longer than polyurethane [7].

- $\quad$ Appropriate alignment of embedded fibers can minimize the stress concentrations in the matrix and at the leaflet/stent interface [44].

- Fiber reinforcement aids leaflet motion and stress concentrations through the transference of the maximum curvature between the basal region and free edge [45].

- $\quad$ SIBS is no more thrombogenic than polyurethane [12].

- Platelet activation potential of the SIBS valve is equivalent to a commercially available bioprosthetic and mechanical valve [46].

- $\quad$ The SIBS valve does not elicit a thrombotic response in vivo [11].

Elliptical and spherical prototype SIBS valves (Figure $7 \mathrm{a}$ and $\mathrm{b}$ respectively) that incorporated individually placed polypropylene fibers (7-0 monofilament polypropylene sutures, Prolene, Ethicon) oriented in the same manner as the natural collagen architecture were manufactured. In vitro hydrodynamic testing was performed on both designs, and they were found to have a valvular pressure gradient and regurgitation equal to or less than that of a St. Jude Bileaflet Mechanical Valve (St. Jude Medical, Minneapolis), proving that short-term functioning was suitable $[9,10]$.

In order to assess polymer fatigue, SIBS dogbone samples with embedded 10-0 monofilament fibers were subjected to cyclic bending and tensile testing. The composite samples were found to have superior tensile fatigue characteristics than polyurethane, and fiber reinforcement was found to improve the bending fatigue properties over isotropic 
SIBS. The actual fiber volume percentage greatly impacted each of these properties, demonstrating the importance of fiber-refinement in the development of a reinforced valve [7].

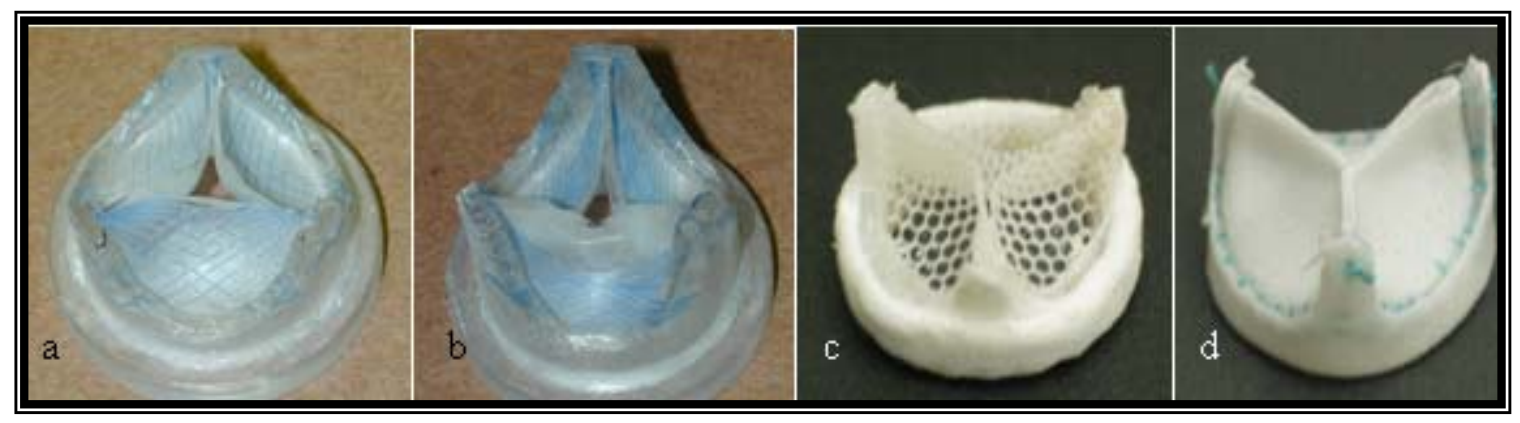

Figure 7. Composite SIBS polymer trileaflet valve designs: (a) Polypropylene embedded spherical valve, (b) polypropylene embedded elliptical valve, (c) LARS ${ }^{\circledR}$ mesh embedded spherical valve, (d) Dacron embedded thermal-form valve.

A finite element analysis (FEA) of the statically loaded polymer trileaflet valve was performed, and parametric analysis revealed that a double ply model led to a reduction in the stress on the polymer matrix. Optimum results were achieved when the fibers were oriented perpendicular to each other and the leaflet/stent interface [44]. The results of the FEA were utilized in the fabrication of valves, where the adoption of a knitted polyester mesh marketed by Boston Scientific/Meadox Medical, Inc. (Oakland, NJ), called LARS ${ }^{\circledR}$ Mesh made it possible for reliable and repeatable orientation of the embedded fibers during fabrication. Polypropylene fibers, that were positioned one at a time, were replaced with the LARS® Mesh to create the composite (Figure $7 \mathrm{c}$ ). The mesh resulted in a deviation from the natural collagen architecture, but hydrodynamic assessment of the valves proved them to have favorable hydrodynamics when compared with a St. Jude Bileaflet Mechanical Valve and a St. Jude porcine bioprosthetic Toronto SPV stentless valve [43]. During in vitro hydrodynamic testing, the transient geometry 
of the leaflet surface was captured using dual camera stereo photogrammetry, and an enhanced numerical method was used to reconstruct the three-dimensional surface in order to quantify changes in leaflet geometry during cyclical motion [45]. The composite LARS ${ }^{\circledR}$ reinforced valve was compared with an isotropic valve, and it was concluded that fiber reinforcement helped leaflet motion by transferring the maximum curvature between the basal region and free edge of the leaflet during cycling. For the nonreinforced valve, the maximum curvature remained in the basal region throughout the cardiac cycle, thereby resulting in a high stress in this critical region.

The thrombogenic potential of SIBS was quantified by measuring platelet deposition with radiolabelled platelets in a parallel plate flow configuration. The SIBS was found to be no more thrombogenic than a medical grade polyurethane approved for cardiovascular applications; however, platelet deposition on SIBS was found to be significantly less than that onto tissue samples extracted from a St. Jude porcine bioprosthetic Toronto SPV stentless valve [7]. This unexpected result prompted further analysis of platelet activation potential under mock physiological conditions in a left ventricular assist device [46]. Platelet activation potential was compared with a St. Jude Bileaflet Mechanical Valve and a St. Jude porcine bioprosthetic Toronto SPV stentless valve, and no significant differences existed. Furthermore, chronic (20 weeks) in vivo studies are being performed in an ovine model, where safety and hemodynamic performance of valves implanted in the aortic position are of interest. In an animal that survived 10 weeks, no sign of thrombus or embolus formation was found, further supporting the valve's biocompatibility [11]. In this particular animal, chronic regurgitation as a result of mechanical failure of the valve was the cause of death, which 
prompted a design change from the LARS ${ }^{\circledR}$ embedded leaflet (Figure 7c) to a Dacron embedded thermal-formed valve (Figure 7d). Preliminary in vivo evaluation of this design has shown tissue ingrowth and deposition to be a factor in valve failure, but thrombosis and embolic complications are still absent.

The preliminary studies have proven that a SIBS trileaflet valve can be fabricated with suitable hemodynamics and thrombogenicity, and fiber reinforcement can be effectively used to improve the fatigue resistance of SIBS by minimizing stress concentration in the matrix. Although preliminary in vivo evaluation of the Dacronreinforced valve revealed tissue ingrowth, contributing to stenosis and valve failure, the LARS ${ }^{\circ}$-reinforced valve did not experience this. It is hypothesized that surface quality and hemodynamics contributed to this tissue ingrowth, so valve design can eradicate this

problem. What can be concluded from these preliminary tests is that both the design and material show promise, and continued experimentation is necessary to determine its success. Further valve development is required to produce a valve that can sustain 15 years of use (600 million cycles).

\subsection{Valve Characterization Methods}

\subsubsection{Hydrodynamic Testing}

In vitro hydrodynamic testing is a key element in the evaluation of performance for a prosthetic valve [41]. The ISO 5840:2005 and FDA standards stipulate the specifics for testing equipment and conditions, thereby providing stringent parameters to assess acute valve function. 
In vitro evaluation of an aortic valve only requires replication of the systemic circulation, including the left atrium, left ventricle, and the large and small arteries. The left ventricle pumps oxygenated blood into the systemic arteries, which progressively decrease in size as they branch. The large arteries offer no resistance to flow, and can be considered as compliance elements, whereas pressure drops across the small arteries are high, defining them as resistance elements. Blood is routed back to the left ventricle through the left atrium, which acts as a reservoir for the system. As a result, the systemic circulation can be represented by a reservoir (left atrium), a pump (left ventricle), compliance elements (aortic (characteristic) and peripheral (systemic) compliance), and a resistance element (peripheral resistance) (Figure 8). A system composed of these elements and capable of reproducing physiological flow characteristics is referred to as a "Pulse Duplicator".

A pulse duplicator is required to have a valve chamber with relevant dimensions, thereby replicating the hemodynamic characteristics across the valve. The system must be capable of producing pressure and flow waveforms that approximate a range of physiological conditions from rest to exercise, and the arrangement of resistance and compliance elements must simulate the arterial tree in the human body in order for the hydrodynamic evaluation to hold any significance. Pressure and flow measurements are the primary determinants of valve performance, and hydrodynamic performance is evaluated with respect to the following parameters:

- Stroke volume: the volume of fluid flowing through a valve in the forward direction during one cycle (Figure 9). 
- Regurgitation: The volume of fluid that flows through a valve in the reverse direction during one cycle; it is the sum of the closure volume and leakage volume and is expressed as percentage of the stroke volume (Figure 9).

- Cardiac output: The mean flow through a valve per minute.

- Mean systolic pressure difference: the average value of the pressure difference (ventricle pressure - aortic pressure (Figure 10)) across a valve during the systolic ejection period.

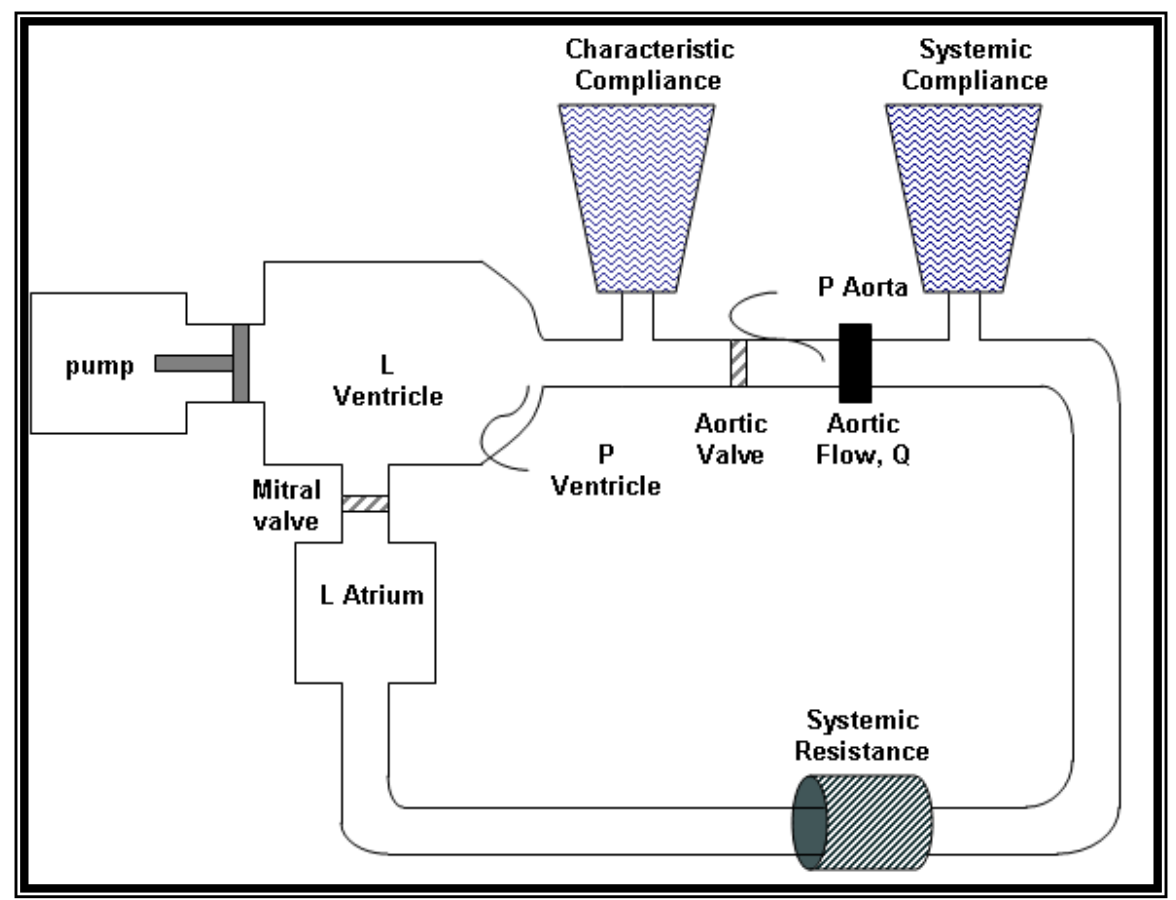

Figure 8. Hydrodynamic function tester for prosthetic heart valves is shown. The fluid mechanical environment is monitored with direct pressure transducers and flow probes.

Due to the variability between test systems, direct comparisons between valve performance on different pulse duplicator systems is not possible; therefore, comparative evaluation with commercially available prototypes is necessary for complete 
hydrodynamic assessment of prototype valves. Nevertheless, minimum performance requirements for prosthetic valves have been provided in ISO 5840:2005 (Table 1).

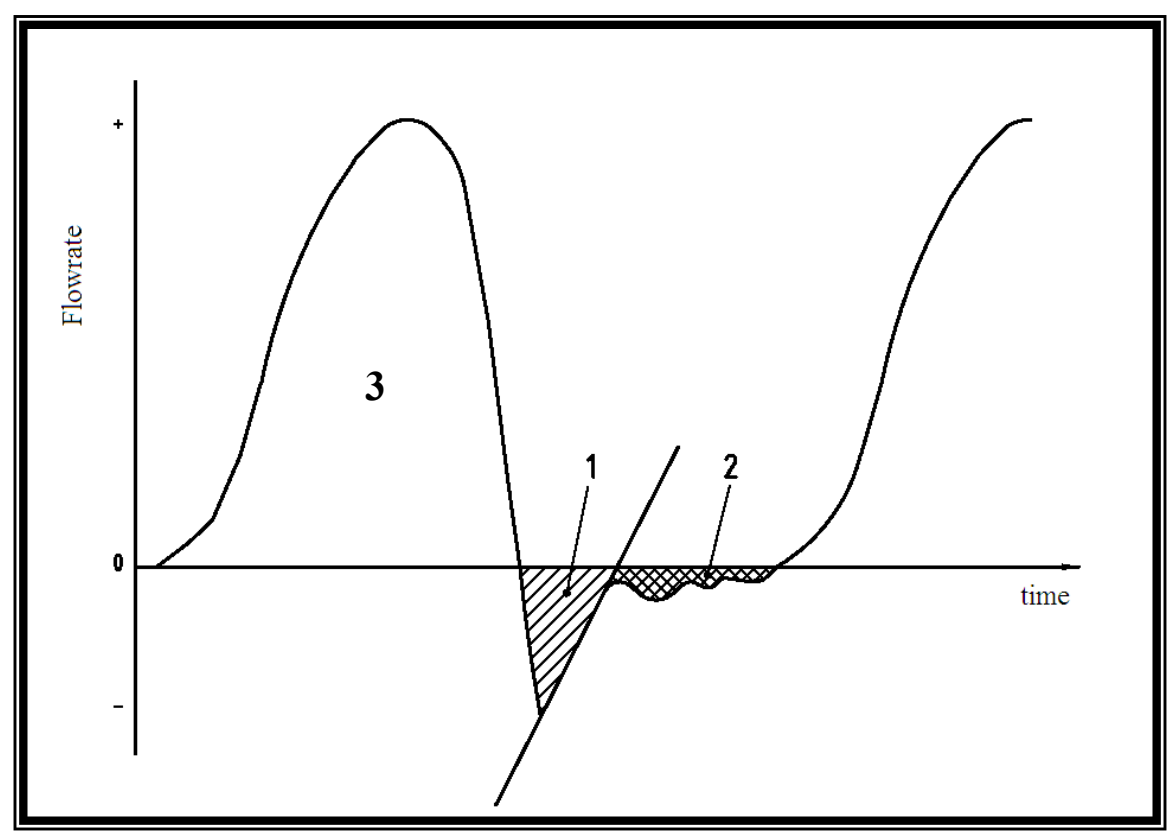

Figure 9. Representative flow waveform where (1) represents the closing volume, (2) represents leakage volume, and (3) represents the stroke volume (ISO 5840:2005).

Table 1. Minimum performance requirements for aortic valve prostheses (ISO 5840:2005).

\begin{tabular}{|c|c|c|c|c|c|c|c|}
\hline Parameter & \multicolumn{7}{|c|}{ Value } \\
\hline Valve Size (TAD, mm) & 19 & 21 & 23 & 25 & 27 & 29 & 31 \\
\hline$\overline{A_{\mathrm{EO}}\left(\mathrm{cm}^{2}\right)}$ & $\geq 0.70$ & $\geq 0.85$ & $\geq 1.00$ & $\geq 1.20$ & $\geq 1.40$ & $\geq 1.60$ & $\geq 1.80$ \\
\hline Regurgitant Fraction (\%) & $\leq 10$ & $\leq 10$ & $\leq 10$ & $\leq 15$ & $\leq 15$ & $\leq 20$ & $\leq 20$ \\
\hline
\end{tabular}




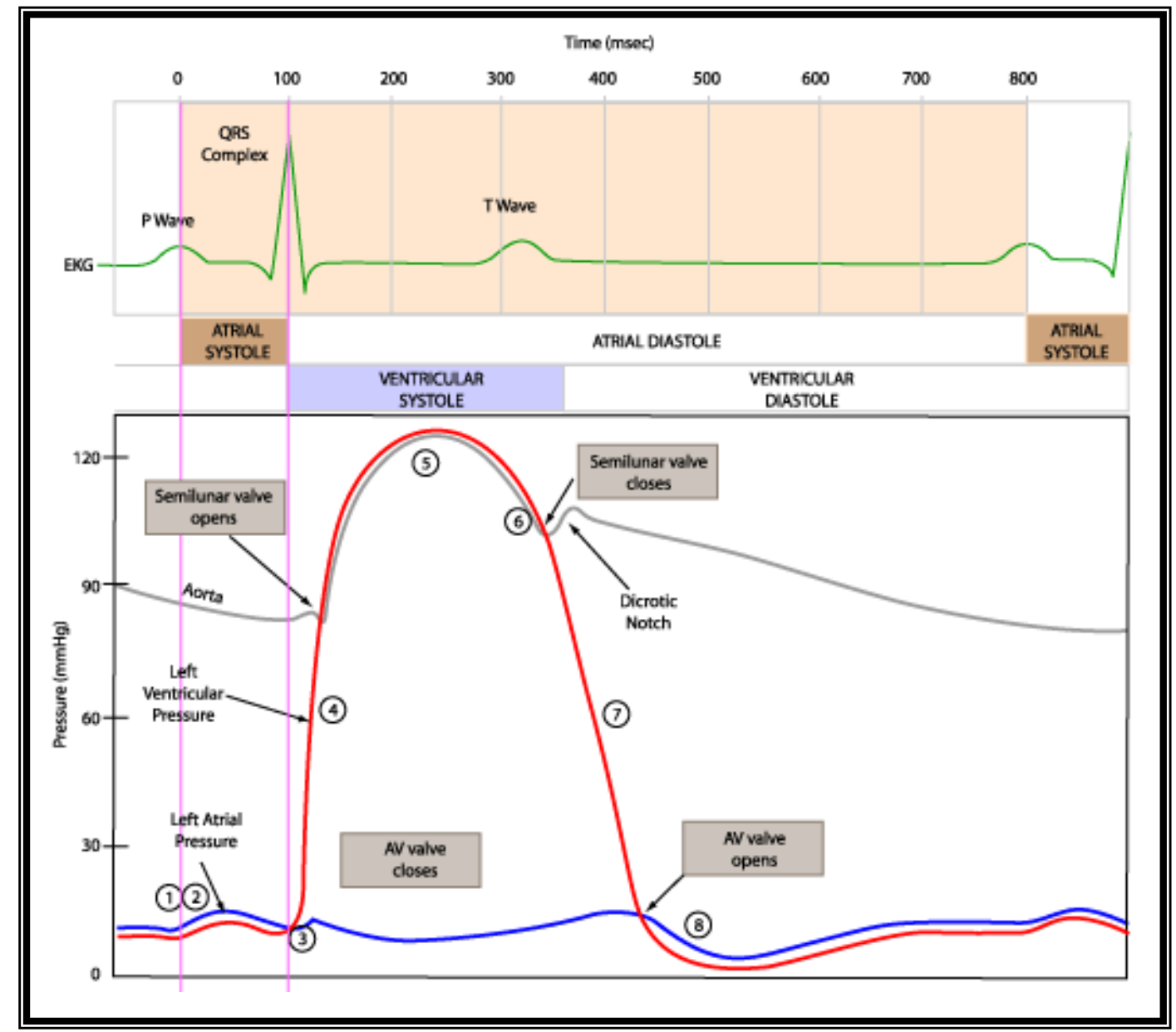

Figure 10. Simultaneous tracings of the left ventricular and aortic pressure waveforms (math.bd.psu.edu/faculty/stevens/PVpresentation.ppt)

In the above table, the effective orifice area $\left(\mathrm{A}_{\mathrm{EO}}\right)$ can be derived as follows:

\section{Equation 1. Effective orifice area.}

$$
A_{E O}=\frac{Q_{v . r m s}}{51.6 \times \sqrt{\frac{\Delta P}{\rho}}}\left(\mathrm{cm}^{2}\right), \text { where: }
$$

$\Delta \mathrm{P}: \quad$ mean pressure difference during forward flow $(\mathrm{mmHg})$,

$\rho: \quad$ the density of the test fluid $\left(\mathrm{g} / \mathrm{cm}^{2}\right)$,

51.6: a constant derived from the Bernoulli equation, and 
Equation 2. Root mean squared forward flow.

$$
Q_{v, r m s}=\sqrt{\frac{\sum_{i=1}^{N} Q_{i}^{2}(t)}{N}}(\mathrm{~mL} / \mathrm{s}), \text { where: }
$$

$\mathrm{Q}_{\mathrm{i}}(\mathrm{t})$ : flow data points $(\mathrm{mL} / \mathrm{s})$,

$\mathrm{i}=1: \quad$ start of systole

$\mathrm{i}=\mathrm{N}$ : end systole

\subsubsection{Accelerated Fatigue Testing}

It becomes necessary to estimate valve longevity in order to evaluate the potential risks and failure modes associated with a prosthesis. Durability testing of heart valve prostheses is used to assure that rigid heart valve substitutes remain functional for over 400 million cycles (10 years) and flexible heart valve substitutes remain functional for over 200 million cycles (5 years).

The FDA and ISO 5840:2005 standards require that a minimum peak pressure difference of $90 \mathrm{mmHg}$ must be established across the closed aortic valves for at least 5\% of each cycle, and this should be maintained for $95 \%$ or more of all test cycles. The rate of cycling is dependent on two primary factors: (1) the potential for complete valve range of motion and (2) the time-dependent behavior of the particular valve materials. Weekly checks are required to verify the transvalvular pressure and overall valve quality, and more quantitative assessment is required approximately every 50-75 million cycles to examine for wear, cracking, and general degradation of the valve in addition to hydrodynamic performance. 
In a multi-station fatigue tester, it is critical that a comparative assessment of valve durability be carried out due to the sensitivity to testing conditions. According to Iwasaki et al. [47], valve lifetime varies according to cycling rate, even when the maximum transvalvular pressure difference is constant; therefore, maintaining strict controls over test conditions is necessary for result correlation. Specifying a pressure difference across a valve does not guarantee that tests are carried out under equivalent conditions [48].

\subsection{Finite Element Analysis (FEA)}

Prior to the availability of computer modeling, knowledge of the failure modes associated with prosthetic valves was gained through in vitro and in vivo use, and changes to designs were made only after problems arose. The downfall to this technique is that a prototype is required for testing, which can make development both time consuming and costly if numerous design iterations are required [49]. Finite element modeling (FEM) can provide a design tool to eradicate certain questionable designs without the need for rigorous prototyping and testing. FEA incorporates a computer model of a material and/or design that is stressed and assessed for specific criteria. It can be used to verify that a certain product meets specifications and can function without failure for the desired product life. The finite element method is a numerical technique in which the governing differential equations can be approximated for solution; as a result it can be applied to multiple physical phenomena including stress, vibration, deflection, and heat conduction, to name a few [50,51]. Discretization of the body into finite elements is performed, and approximations are carried out over each finite element. 
The solution for each of the elements is summed to arrive at a solution for the entire body.

Computational modeling provides a means to establish the structure-function relationships in valves: numerical simulations have provided a wealth of knowledge in the area of stress distribution and design optimization for trileaflet heart valves [52], where key features contributing to the stress concentrations in valve leaflets include valve geometry, leaflet anisotropy, and supporting stent structure. Most models do not represent the behavior of the valve leaflet throughout the entire cardiac cycle as complex interactions between the flow and leaflets exist. Loading of the valve can be achieved by either applying a differential pressure across the leaflets or by stretching the boundaries of the leaflets.

FEM applied to bovine pericardial valves has shown that the primary stress concentrations occur at the commissure point close to the top of the stent post [51]. The extent of this stress is highly dependent on leaflet anisotropy, stent flexibility, and the angle that the free edge forms with the stent post. In the aortic allograft and porcine xenograft on the other hand, the primary stress concentration is in the belly region, and stress at the coaptations and commissures are insignificant [51]. This phenomenon is believed to be as a result of the collagen fiber orientation. In the aortic allograft, leaflet strains in the radial direction are four times greater than those in the circumferential direction, a fact consistent with the natural collagen and elastin fiber layout. These strains are decreased in porcine xenografts, as glutaraldehyde fixation leads to leaflet stiffening. 
Fiber/collagen reinforcement in trileaflet valves has been proven to contribute to the minimization of stress concentrations and improvement to the dynamic characteristics of leaflets $[29,30,37-39,44,41,52,53]$, but the impact of varying fiber orientation has received only a cursory glance. De Hart et al. [29] performed a three-dimensional FEA on a fiber-reinforced stented polymer trileaflet valve with either a sinusoidal or uniform circumferential fiber orientation. The leaflet was deformed from a stress-free open configuration to a closed configuration by the application of a uniform pressure load on the aortic side. There was an overall decrease in stress concentrations in the primary high stress locations (commissures) when compared with non-reinforced samples, and the presence of fiber reinforcement created a more homogeneous stress distribution in the ventricular and aortic polymer layers. Numerical instabilities required the use of nonphysiologic models in this evaluation. FEA performed by Arcidiancono et al. [53] on the Pericarbon ${ }^{\mathrm{TM}}$ aortic tricuspid valve revealed that pericardial orthotropy has a marked impact on leaflet dynamics, dependent on the particular orientation of the pericardium during valve manufacture. The orientation of the pericardial tissue affects both leaflet dynamics and stress distribution and is presumed to significantly affect the durability and function of pericardial prostheses. Load-pressure curves in conjunction with pericardial mechanical properties were applied in this dynamic model without the consideration of either the stent behavior or blood interaction. In both de Hart's and Arcidiancono's model, a rigid stent body was considered. Cacciola et al. [30] adapted de Hart's model for a stentless fiber-reinforced polymer trileaflet valve. A 75\% reduction in stress when compared with the stented model was achieved. Higher stress concentrations in the 
stented valve were believed to be caused by the unphysiological flexure experienced by leaflets constrained in a rigid stent.

For a material subjected to cyclic loading, the number of cycles to failure is controlled by the maximum stress level. High stress concentrations are associated with the failure locations in trileaflet valves [51]; therefore, the knowledge of stress concentrations is imperative to the design of a trileaflet valve capable of withstanding cyclic fatigue.

\subsection{Fatigue Modeling}

Fatigue is referred to as the onset of failure as a result of cyclic loading [42, 54]. During the design of heart valves, it is imperative to know the number of cycles to failure in order to ensure a device's durability. In vitro testing can be both time consuming and costly, and the particular cycling rate chosen for testing in accelerated heart valve testers and tensile testers is known to have a marked effect on the number of cycles to failure $[47,48,42,55]$. As a result, more efficient fatigue predictive methods are needed, and fatigue life prediction models have been developed for this purpose.

The most common approach and the first step in the prediction of fatigue failure involves stress-versus-life (S-N) curves $[42,54,56]$. In this method, the number of cycles required to induce fatigue failure of a specimen is estimated at multiple alternating $\left(\sigma_{\mathrm{a}}\right)$ and mean $\left(\sigma_{\mathrm{m}}\right)$ stress combinations, where the fatigue strength of the material refers to the stress on an S-N curve for a particular life of interest $\left(\mathrm{N}_{\mathrm{f}}\right)$. In some cases, the loglinear plot of S-N data approximates a straight line that can be represented by the equation [42]: 


\section{Equation 3}

$S=\sigma_{a}+m * \log \left(N_{f}\right)$, where:

$\sigma_{\mathrm{a}}:$ amplitude of stress

$\mathrm{S} \& \mathrm{~m}$ : fitting constants, where $\mathrm{S}$ is the $\mathrm{Y}$-intercept and $\mathrm{m}$ is the slope

$\mathrm{N}_{\mathrm{f}}$ : number of cycles to failure at a particular applied stress

Mouritz [56] utilized this linear relationship in the development of a simple fatigue-life model for three-dimensional (3-D) fiber-polymer composites. In this particular micromechanical model, the properties of 3-D composites were approximated from the knowledge of 2-D fatigue-life models, where three empirically determined constants were required: (1) the ultimate strength of the 3-D composite $\left(\sigma_{3 \mathrm{D}}\right),(2)$ the ultimate strength of the 2-D laminate $\left(\sigma_{2 \mathrm{D}}\right)$, and $(3)$ the slope $(\mathrm{m})$ parameter of the fatigue-life $(\mathrm{S}-\log \mathrm{N})$ curve for the 2-D laminate. The simplified relationship from Equation 3 was adjusted to account for the fact that the 2-D data was used for approximation of fatigue-life of 3-D composites, providing the following relationship:

\section{Equation 4}

$$
S_{3 D}=\frac{\sigma_{3 D}}{\sigma_{2 D}}\left(\sigma_{2 D}+m * \log \left(N_{f}\right)\right)
$$

Upon evaluation of the tensile fatigue properties of the 3-D composite, there was a good correlation between experimental data and model prediction for S-N, showing the validity of the method in this particular instance. The model, however, is only applicable to materials that have linear $\mathrm{S}-\log \mathrm{N}$ curves, and it is only valid for comparison under 
equivalent testing conditions. Extrapolation of the model to varying cycling rates or loading conditions was not evaluated. Many materials do not exhibit a linear S-logN relationship, instead the S-N curve begins to flatten and reaches a threshold stress amplitude, below which failure does not occur. Materials that exhibit these tendencies cannot utilize the simplistic approach presented above.

Cacciola [41] and Huang [55] utilized the theory of continuum damage mechanics, where the dynamic fatigue of the material results in progressive degradation, thereby affecting the sample's response to stress and ultimately leading to failure. According to Cacciola, damage can be defined as:

\section{Equation 5}

$D=\frac{a-\widetilde{a}}{a}$, where

a: the area of a section through the element

ã: the effective area for stress transfer (a - area occupied by microcracks and damage)

When $\mathrm{D}=1$, the sample has failed, and when $\mathrm{D}=0$, the sample is undamaged. With this definition of damage, the damage along a specimen is initially zero, but cyclic loading results in a progressive increase in damage that changes the material properties and affects the number of cycles the material can sustain until failure $\left(\mathrm{N}_{\mathrm{f}}\right)$. Iterative application of this in a finite element model was proposed for fatigue-life prediction of a polymer trileaflet valve, but thus far it has only been used to evaluate non-reinforced EPDM rubber. In this particular evaluation, a hole was created in the isotropic EPDM 
specimen, and it was exposed to cyclic fatigue. The sample failed at 148 cycles in the experimental evaluation, while the simulation attained $\mathrm{D}=1$ after only 87 cycles. Future work on this model involves program adaptation for the incorporation of large displacements, and the final implementation for a polymer trileaflet heart valve.

Cacciola's definition of damage or fatigue is reminiscent of the Palmgren-Miner Rule, which states that fatigue failure is expected when life fractions sum to unity:

\section{Equation 6}

Fatigue Failure $=\sum \frac{N_{j}}{N_{f j}}=1$, where

$\mathrm{N}_{\mathrm{j}}$ : number of cycles the stress amplitude, $\sigma_{\mathrm{aj}}$, is applied

$\mathrm{N}_{\mathrm{fj}}$ : Number of cycles to failure when stress amplitude, $\sigma_{\mathrm{aj}}$, is applied

The Palmgren-Miner Rule is applicable when a device is subject to variable amplitude loading, and the effects of each are summed for the approximation of fatigue life. For both Cacciola's and the Palmgren-Miner approach, initial knowledge of the S-N relationship is required for the evaluation.

Huang [55] adopted a more complex relationship for the prediction of fatigue in a fabric composite that was subjected to biaxial loads. A Bridging micromechanics model was used for the simulation of fatigue strength and S-N data for a woven fiber-reinforced composite was used as input. In this approach, the fiber and matrix properties were required in addition to the S-N data for the specific cyclic conditions evaluated. In the model, the unit cell of the woven composite was subdivided into unidirectional slices, and the Bridging model was used to relate the internal stresses on the fiber and matrix 
with the load on the unidirectional composite. The stresses obtained from the Bridging model were evaluated against critical values from the S-N data, and when any constituent reached this critical parameter, the composite was considered to reach failure. The Bridging model itself is complex, and discussion of the mathematical formulation serves no purpose in this present evaluation, suffice to say, the Bridging model was used to correlate the volume averaged stress increments in a unidirectional composite. Once assembled, it can be applied in a fatigue-life model similar to Cacciola's, where damage accumulation ultimately resulted in fatigue failure of the composite. The total stress accumulation due to cyclic load was approximated as:

\section{Equation 7}

$\left[\sigma_{i j}\right]^{K+1}=\left[\sigma_{i j}\right]^{K}+\left[\sigma_{i j}\right]$, where

$\left[\sigma_{\mathrm{ij}}\right]^{(0)}$ : the initial residual stresses in the constituents

Fatigue was defined when:

\section{Equation 8}

$\left[\sigma_{i j}\right]^{K+1}=\sigma(R, N, \omega) \geq \sigma_{u}(R, N, \omega)$, where

$\mathrm{R}$ : ratio between the minimum and maximum stresses

$\mathrm{N}$ : cycle number

$\omega:$ frequency

$\sigma_{\mathrm{u}}$ : fatigue limit at $\mathrm{R}, \mathrm{N}$, and $\omega$ 
The model was evaluated against experimental results obtained in literature, where the correlation between each was not convincing. The true validity of this model can only be evaluated once a complete data set of experimental results is available for comparison. 


\section{SPECIFIC AIMS AND HYPOTHESES}

The two primary challenges preventing the commercial success of polymer trileaflet heart valves include polymer selection and valve design. Many of the polymers chosen for previous designs of trileaflet valves have not been biostable, where oxidation led to embrittlement of the polymer, making it less resistant to cyclic fatigue [7]. Materials that are more resistant to oxidation are said to be less vulnerable to enzymatic degradation and are, therefore, more biostable. The adoption of SIBS for the polymer valve is proposed to overcome the problem of biostability, where preliminary testing has proven it to be stable in both in vitro [8] and in vivo [11, 14] oxidative environments. The high dynamic tensile and bending stresses acting on leaflets cause mechanical degradation, resulting in the formation of cracks and tears. These cracks have been proven to be sites for calcification in polyurethane valves, but ultimately, propagation of these deformities causes catastrophic valve failure. It is hypothesized that this problem can be overcome by an appropriate valve design, such as the adoption of a valve geometry and reinforcement that will effectively reduce the high stress concentrations in the leaflet polymer matrix, thereby reducing crack formation. High stress is known to be a primer for degradation in materials exposed to cyclic fatigue [42]. It is this aspect of valve design that is still an issue for the SIBS valve; as a result, the focus of this work was the impact of design on both the acute valve function and long-term durability.

Questions that were addressed included:

\section{A. What is the best possible leaflet design that will result in optimal acute and long- term function of a polymer trileaflet valve?}

\section{B. How important is stent flexibility to the function of a trileaflet valve?}




\section{Can a simplified fatigue-prediction model be incorporated into the development process for a polymer trileaflet valve; will it adequately predict the durability of such a complex device?}

In order to answer these questions, the following three specific aims were carried out:

1. SIBS Valve Characterization: This investigation characterized different valve prototypes in terms of leaflet and stent tensile properties, assembled valve hydrodynamic function, and overall valve quality. The tensile properties of the valve components were evaluated on an Electroforce ${ }^{\mathrm{TM}}$ (ELF) 3200 materials tester (Bose Corporation, Electroforce Systems Group, Minnetonka, MN) by means of static tensile and Poisson's ratio tests; hydrodynamic evaluation was performed on a Vivitro Systems Left Heart and Systemic Circulation Simulator; and SEM was used to assess leaflet quality. The tensile, Poisson's ratio, and hydrodynamic properties were required for input into both the FEM and, therefore, the fatigue lifetime prediction model.

2. Finite Element Modeling: Based on the literature review, it was determined that the three key parameters affecting leaflet stress concentrations include the supporting structure (stent) flexibility, the reinforcement alignment (leaflet anisotropy), and the geometry of the leaflet, especially in the region of coaptation. Valve models including varying degrees of stent flexibility, coaptation and/or leaflet geometry, and fiber reinforcement were evaluated by means of a finite element model to determine their impact on leaflet stress concentrations. By making iterative changes to the 
parameters above, one can determine which has the greatest benefit in terms of stress reduction.

3. Durability Assessment and Fatigue Prediction: This investigation was used to characterize the valve components and completed valve prototypes from Aim 1 in terms of their durability. The summation of this research was a mathematical model that is proposed as a tool for the fatigue lifetime prediction of polymer trileaflet valves. Dynamic tensile fatigue tests of leaflet samples were characterized on the ELF tester, and the resultant S-N relationship were applied in the fatigue lifetime prediction model. The final model was validated by both the S-N data and accelerated fatigue data for valves evaluated on the Vivitro Systems Hi-Cycle Accelerated Fatigue Tester.

All three Specific Aims are interrelated, where the output of Aim 1 was necessary for input into Aim 2, and the output of both Aims was required for input into Aim 3. The culmination of the research was a mathematical model from Aim 3 that has the potential to be implemented to predict the fatigue lifetime of the valve, negating the need for a slew of time-consuming durability tests. Ultimately, in vitro accelerated fatigue testing is required for valves intended for clinical application as specified by the FDA and ISO standards. 


\section{METHODOLOGY}

As mentioned previously, the three primary aspects of the valve that were under investigation were the stent flexibility, leaflet anisotropy, and leaflet/coaptation geometry. In order to investigate the impact that these parameters had on both the acute and long-term function of the valve, the variables in Table 2 were applied to different designs that were subject to experimentation and/or modeling (Specific Aims $1-3$ ). In the table below, the combination of leaflet reinforcement and manufacturing procedure manipulates the anisotropic properties of the leaflet, while the combination of stent geometry and polymer type manipulates the stent flexibility.

Table 2. Listing of all potential candidates for manufacture and testing of a trileaflet heart valve

\begin{tabular}{|c|c|c|c|c|c|}
\hline & \multicolumn{3}{|c|}{ Leaflet } & \multicolumn{2}{|c|}{ Stent } \\
\hline & Reinforcement & $\begin{array}{c}\text { Manufacturing } \\
\text { procedure }\end{array}$ & $\begin{array}{c}\text { Coaptation } \\
\text { Geometry }\end{array}$ & Material & Geometry \\
\hline 1 & PET Knit 1 (JSI) & Dip-Coating & No Curvature & $\begin{array}{l}30 \% \\
\text { SIBS } \\
\end{array}$ & Low Profile \\
\hline 2 & $\begin{array}{l}\text { PET Knit } 2 \\
\text { (BARD) }\end{array}$ & Hybrid & $\begin{array}{l}\text { Medium } \\
\text { Curvature }\end{array}$ & $\begin{array}{l}48.5 \% \\
\text { SIBS }\end{array}$ & $\begin{array}{l}\text { Medium } \\
\text { Profile }\end{array}$ \\
\hline 3 & PET Weave & Solvent Casting & $\begin{array}{c}\text { High } \\
\text { Curvature }\end{array}$ & & $\begin{array}{l}\text { High } \\
\text { Profile }\end{array}$ \\
\hline 4 & $\begin{array}{l}\text { PET Mesh } 1 \\
\text { (LARS) }\end{array}$ & & & & \\
\hline 5 & $\begin{array}{l}\text { PET Mesh } 2 \\
\text { (XA-47) }\end{array}$ & & & & \\
\hline 6 & $\begin{array}{l}\text { PET Mesh } 3 \\
\text { (NZ-11) }\end{array}$ & & & & \\
\hline 7 & $\begin{array}{c}\text { PET Mesh } 4 \\
\text { (Athletic Mesh) }\end{array}$ & & & & \\
\hline
\end{tabular}




\subsection{Valve Manufacturing Procedure}

A total of four distinct design prototypes were produced, incorporating the components listed in Table 2, in order to investigate the impact of variable stent flexibility and leaflet anisotropy on valvular function. Finite element modeling was used to evaluate the affect of varying leaflet coaptation geometry on the valve stress concentrations; therefore, all the prototypes that were manufactured for the experimental evaluation incorporated the medium coaptation curvature. A summary of the component parameters for the four valve designs is provided in Table 3, and descriptions of their manufacturing procedures are provided in the following sections. The detailed manufacturing protocols are provided in Appendix I.

\subsubsection{Design 1: Pre-formed individual leaflets}

Design 1 prototype valves incorporated the polyester mesh, LARS® (Boston Scientific/Meadox Medical, Inc., Oakland, NJ), and a low profile, 30\% styrene SIBS stent (refer to Figure 106, Appendix II). The nominal thickness of the reinforcement mesh was $0.15 \mathrm{~mm}$, and after coating with three dips in the SIBS solution, an average leaflet thickness of $0.21 \mathrm{~mm}$ was achieved.

Leaflets were manufactured by dip-coating in a $15 \%$ SIBS ( $8.5 \%$ styrene) in toluene solution (Appendix I) as follows: Aluminum leaflet dipping molds were cleaned and buffed to remove any surface debris and scratches. The aluminum molds were dipped into the SIBS/toluene solution and placed in an oven set to $75 \pm 5^{\circ} \mathrm{C}$ for $>30$ minutes. The LARS ${ }^{\circ}$ mesh was cut into 2.5 inch square samples whose edges were parallel to the minimum and maximum elongation of the mesh. The mesh was then secured over the coated mold with an o-ring so that the maximum elongation of the mesh 
was oriented in the circumferential direction. The whole assembly was then dipped two more times into the SIBS/toluene solution providing a three layer coating with the mesh sandwiched between the first and second layer. On the second dip, the molds were allowed to dry at $75 \pm 5{ }^{\circ} \mathrm{C}$ for $>30$ minutes, but on the final dip, the molds were placed in the oven for $>2$ hours to complete the drying process. The leaflets were removed from their aluminum dipping molds and transferred to a holding fixture.

The stent was manufactured by compression molding 30\% styrene SIBS pellets at $180^{\circ} \mathrm{C}$ and 7,000 $\mathrm{lbs}$ in a low profile stent compression mold (Appendix I and II). The additional flash was trimmed from the stent, and the stent was now ready for attachment of the leaflets.

A $15 \%$ SIBS $(8.5 \%$ styrene) in toluene solution was painted onto the area of attachment of both the stent and leaflet, and the leaflet was properly aligned and adhered to the stent. The bonding was repeated for all three leaflets in turn. The leaflets were trimmed to allow the slightly curved coaptation geometry, and the valves were visually inspected: (1) their thicknesses were measured with a Digimatic Outside Micrometer (Mitutoyo, Boca Raton, FL) and recorded, and (2) the leaflets were inspected for bubbles and flaws, their edges were inspected for ragged appearances, and adjacent leaflets were inspected for excessive glue and fusing.

\subsubsection{Design 2: Dip-Coated Cylinder}

The Design 2 prototype valves incorporated one of four polyester reinforcement fabrics and a high profile, 30\% styrene SIBS stent (refer to Figure 106, Appendix II). The reinforcement materials included LARS ${ }^{\text {, }}$, an Athletic Mesh (Lot \# 074-0837, JoAnn Fabric and Craft Stores, Miami, FL), a polyester weave (JoAnn Fabric and Craft Stores, 
Miami, FL), and a Dacron (Jet Set Interlock (JSI), JoAnn Fabric and Craft Stores, Miami, FL). By selecting various different materials for leaflet reinforcement, the impact of varying anisotropic properties could be investigated. For the JSI-Dacron-reinforced leaflets, the impact of changing reinforcement orientation and polymer coating were assessed. Valves were made where the JSI-Dacron was oriented so that the maximum elongation was in either the circumferential or radial direction, and the JSI-Dacron was dip-coated with either a $15 \%$ SIBS ( $8.5 \%$ styrene) in toluene solution or a porous $15 \%$ SIBS (16\% styrene) in cyclopentane solution (refer to Appendix I). For all other leaflet reinforcement materials, the maximum elongation was oriented circumferentially, and the fabric was coated with the $8.5 \%$ styrene SIBS. By increasing the stent profile, greater stent flexibility was achieved when compared with that from Design 1.

Leaflets were fabricated from one of the flat fabric reinforcement materials stated above. The fabric was cut into 3 inch square samples whose edges were parallel to the minimum and maximum elongation of the fabric. The flat sample was then heat sealed into a cylinder, with the maximum elongation oriented in either the circumferential or radial direction, dependent on the specific design. The excess fabric was cut from the heat-sealed joint to allow a clean leaflet tube. The leaflet tube was then dip-coated twice in either the SIBS in toluene or SIBS in cyclopentane solution, and after each dip, the leaflet was dried for 30 minutes in a $60^{\circ} \mathrm{C}$ oven. Once dry, the edges were trimmed to allow a clean, flat edge.

The stent was manufactured according to the same methods as that used for Design 1, except the high profile stent compression mold was used with the $30 \%$ styrene SIBS. 
Table 3. SIBS valve design summary

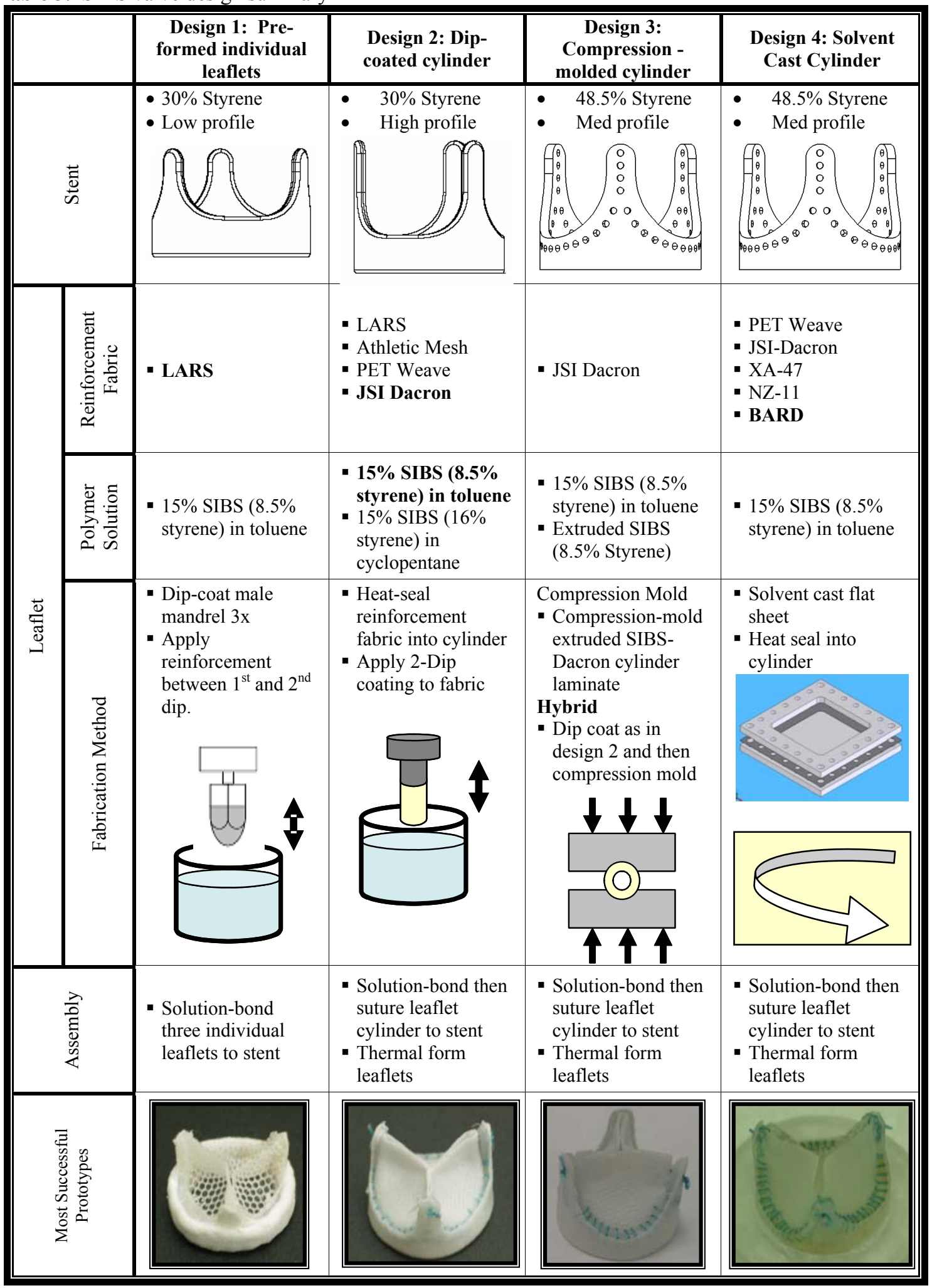


For the leaflet/stent assembly, a 15\% SIBS in toluene solution was painted onto the area of attachment of the stent base, and the leaflet was properly aligned and adhered so that half of the leaflet resided above the stent base and half resided below. The leaflet was then sutured to the stent following the contour of the top edge of the stent, including the stent base and posts. Ethibond Excel suture (5-0, Ethicon, inc., Piscataway, NJ) was used to suture through both the stent and leaflet to allow a tight junction between each. The excess material at the base of the stent was folded up and onto the stent to tightly cover the outer surface. It was trimmed to mimic the outer geometry of the stent, and a coating of $15 \%$ SIBS ( $8.5 \%$ styrene) in toluene was applied to bond the two materials. A reinforcement suture was added at the top of each stent post to secure the outer fabric to the stent. The trileaflet geometry was obtained by thermal-forming the leaflets with aluminum leaflet forming mandrels for 1 hour at $80^{\circ} \mathrm{C}$. The leaflets were trimmed to allow the slightly curved coaptation geometry, and the valves were visually inspected as in Design 1.

\subsubsection{Design 3: Compression-Molded Cylinder}

Design 3 prototype valves incorporated a compression-molded JSI Dacron leaflet mounted on a $48.5 \%$ styrene SIBS, medium profile stent (refer to Figure 106, Appendix II). The leaflet reinforcement was always oriented with the maximum elongation in the circumferential direction, but by applying one of two different compression-molding techniques, leaflets with variable mechanical properties were produced. A medium profile stent consisting of a higher percentage styrene SIBS provided less stent flexibility than that of Design 2. 
Leaflets were fabricated from 3 inch square JSI Dacron samples cut parallel to the minimum and maximum elongation. As in Design 2, the flat fabric sample was heat sealed before the addition of the $8.5 \%$ styrene SIBS polymer. For Design 3, one of two polymer coating techniques was applied (refer to Appendix I), namely: 1) Compression molded laminate or 2) Compression-molded hybrid. For the compression-molded laminate, a true 3 layer laminate was produced by compression-molding two outer layers of $8.5 \%$ styrene SIBS into a cylinder and compressing the JSI cylinder between these two layers at $200^{\circ} \mathrm{C}$ and $30,000 \mathrm{lbs}$. For the hybrid design, the JSI Dacron leaflet cylinder was dip-coated twice, as in Design 2, and this was then subjected to compression molding in a cylinder clam-shell mold at $200^{\circ} \mathrm{C}$ and 3,000 lbs.

The stent was manufactured according to the same methods as that used for Designs 1 and 2, except the medium profile stent compression mold was used with the 48.5\% styrene SIBS. After compression molding, the stent suture holes were drilled into the stent following a suture hole template. Suture holes were required in the higher styrene content stent as it was too hard to suture through by hand.

The leaflet/stent assembly was the same as that for Design 2.

\subsubsection{Design 4: Solvent Cast Cylinder}

Design 4 prototype valves incorporated one of 4 leaflet reinforcement fabrics and the $48.5 \%$ styrene SIBS, medium profile stent from Design 3. The reinforcement materials included a polyester weave (JoAnn Fabric and Craft Stores, Miami, FL), a Dacron (Jet Set Interlock (JSI), JoAnn Fabric and Craft Stores, Miami, FL), one of two polyester meshes (XA-47 or NZ-11, Apex Mills, Inwood, NY), and a polyester knit (P04081, BARD Peripheral Vascular, Inc., Tempe, AZ). Once again, varying the leaflet 
reinforcement allowed the evaluation of varying anisotropic leaflet properties on valvular function. For the PET weave and the XA-47 reinforcement, the principal fiber directions were oriented $45^{\circ}$ between the circumferential and radial directions; these two reinforcement types had the same mechanical properties in the orthotropic direction due to the orientation of the fibers. For all other materials, the maximum elongation was oriented in the circumferential direction. The JSI Dacron leaflet was manufactured by dip-coating, as in Design 2, but all other leaflets were manufactured by the solvent casting method.

A casting plate was designed for the solvent casting technique (refer to Appendix I) that consisted of a bottom plate, a top plate, and a gasket (Figure 11). The reinforcement fabric was secured between the top and bottom plates, above the gasket, and a controlled volume of $15 \%$ SIBS ( $8.5 \%$ styrene) in toluene solution was poured into the plate to completely coat the fabric surface. The gasket was used to lift the reinforcement fabric off the bottom plate, thereby allowing uniform coating on both sides of the material. This fabrication technique was found to efficiently coat the fabric with a thin layer of SIBS. The underlying fabric was completely coated with no exposed fibers, and the fabric was efficiently placed in the center of the polymer matrix, as can be seen from the SEM images (Figure 12). The flat, SIBS-coated leaflet samples were then heatsealed into a leaflet tube as in Designs 2 and 3, with the exception that they were already coated with the polymer prior to heat-sealing. The stent fabrication and valve assembly techniques remained the same as that for Design 3.

All prototype valves and valve components were subjected to the testing outlined in Specific Aims $1-3$. 


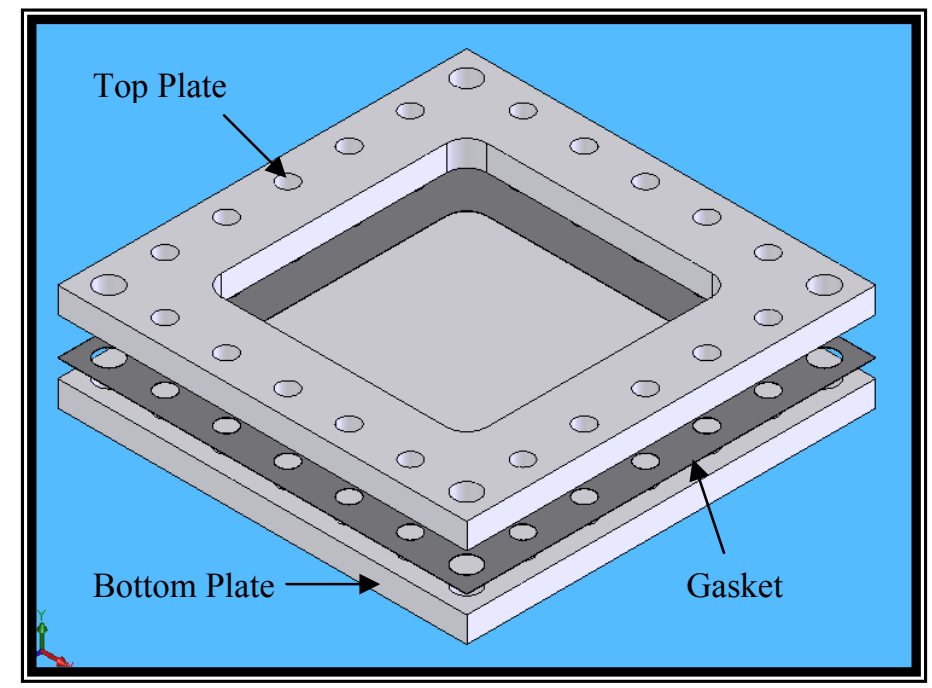

Figure 11. Casting plate for the uniform coating of leaflet reinforcement fabrics

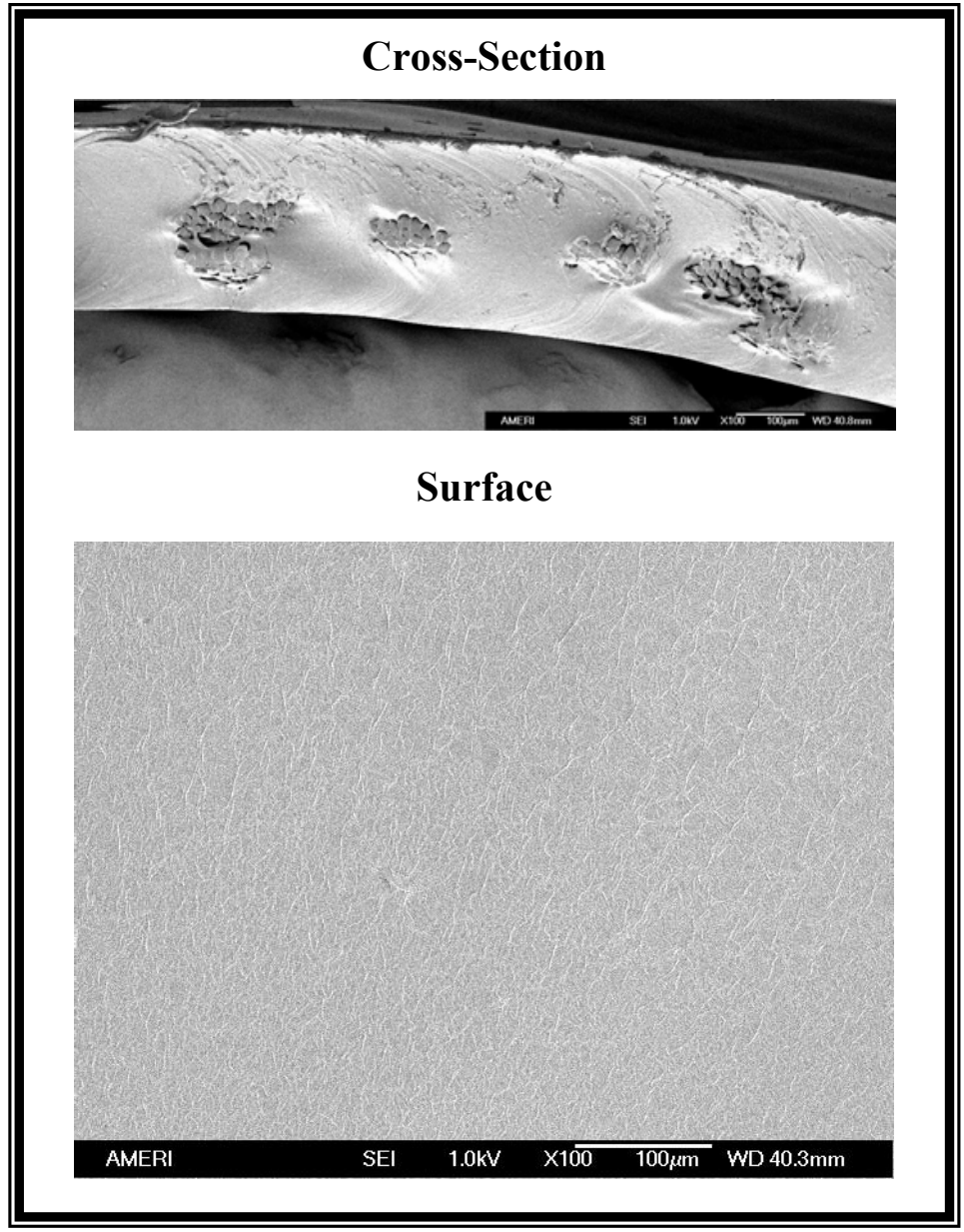

Figure 12. SEM images of a solvent cast PET weave sample showing the cross-sectional and surface views. It is apparent from the cross-sectional view that the fiber reinforcement is embedded in the center of the SIBS matrix, and that the exposed polymer surface is smooth with no fiber exposure. 


\subsection{Specific Aim 1 - Valve characterization}

The purpose of this Aim is to characterize the valve and each of its components (leaflets and stent) to assess how the component tensile properties affect the function of the valve as a whole. Iterative adjustments to valve geometry, reinforcement technique, and stent stiffness and design are characterized in terms of their impact on valve hydrodynamics. In addition, the tensile properties and Poisson's ratio measurements obtained were used to define the material properties in the finite element model of Aim 2, and the transvalvular pressure drop versus time curve obtained from the hydrodynamic analysis was used to specify the loading regimen for the finite element model.

\subsubsection{Tensile Testing}

When evaluating the impact of material properties on valvular function, the tensile properties of both the leaflets and stent are important. During diastole, a backpressure of approximately $90 \mathrm{mmHg}$ is acting on the valve, placing the leaflets in tension, and this tensile force is transferred to the stent at the commissure. During systole, the outward flexion of the stent can aid in the opening of the leaflets. For this reason, tensile testing of both the heart valve leaflets and stent is needed to fully characterize the impact of leaflet and stent design on valve function.

The properties in both the radial and circumferential directions are important in determining valve integrity during diastole. Radial compliance of the leaflets allows them to stretch in the radial direction and coapt to prevent regurgitation. A valve that is too stiff radially will not seal properly, resulting in regurgitation. The primary tensile load acting on the leaflets during diastole is oriented in the circumferential direction, so the tensile properties circumferentially verify whether or not a leaflet can withstand the 
diastolic load. As a result, leaflet samples were fabricated in the same manner as for valve fabrication (refer to Table 3), and circumferential and radial samples were cut and tested for comparison with each other leaflet prototype (listed in Table 4). Stent samples were manufactured by compression molding, which is the same technique used to manufacture actual stents.

Table 4. Valve leaflet and stent components that were subjected to tensile testing (refer to section 4.1 for the valve manufacturing procedure).

\begin{tabular}{||c|c|c|c|}
\hline $\begin{array}{c}\text { Valve } \\
\text { Component }\end{array}$ & $\begin{array}{c}\text { Reinforcement } \\
\text { Material }\end{array}$ & Polymer & $\begin{array}{c}\text { Manufacturing } \\
\text { Procedure }\end{array}$ \\
\hline \multirow{6}{*}{ Leaflet } & PET Knit 1 (JSI) & $8.5 \%$ Styrene SIBS & $\begin{array}{c}\text { Design 2: Dip- } \\
\text { Coating }\end{array}$ \\
\cline { 2 - 4 } & PET Knit 1 (JSI) & $8.5 \%$ Styrene SIBS & Design 3: Hybrid \\
\cline { 2 - 5 } & PET Knit 2 (BARD) & $8.5 \%$ Styrene SIBS & $\begin{array}{c}\text { Design 4: Solvent } \\
\text { Casting }\end{array}$ \\
\cline { 2 - 5 } & PET Weave & $8.5 \%$ Styrene SIBS & $\begin{array}{c}\text { Design 4: Solvent } \\
\text { Casting }\end{array}$ \\
\cline { 2 - 5 } & PET Mesh 2 (XA-47) & $8.5 \%$ Styrene SIBS & $\begin{array}{c}\text { Design 4: Solvent } \\
\text { Casting }\end{array}$ \\
\cline { 2 - 5 } & PET Mesh 3 (NZ-11) & $8.5 \%$ Styrene SIBS & $\begin{array}{c}\text { Design 4: Solvent } \\
\text { Casting }\end{array}$ \\
\cline { 2 - 5 } & & $8.5 \%$ Styrene SIBS & $\begin{array}{c}\text { Compression } \\
\text { Molding }\end{array}$ \\
\hline \multirow{6}{*}{ Stent } & & $30 \%$ Styrene SIBS & $\begin{array}{c}\text { Compression } \\
\text { Molding }\end{array}$ \\
\cline { 2 - 5 } & & $48.5 \%$ Styrene SIBS & $\begin{array}{c}\text { Compression } \\
\text { Molding }\end{array}$ \\
\hline
\end{tabular}

Tensile testing was carried out on the Electroforce ${ }^{\mathrm{TM}}$ (ELF) 3200 materials tester (Bose Corporation, Electroforce Systems Group, Minnetonka, MN), following ASTM standards D 638M - 89 (plastics), D $882-88$ (thin plastic sheets), and D $3039-89$ (composites). A crosshead speed of $5 \mathrm{~mm} / \mathrm{min}$ was used in accordance with these standards. Outcome measures included: Young's Modulus (E), ultimate tensile stress 
(UTS), and ultimate strain (US), where pertinent. A minimum of five specimens was tested for each, and the standard deviation was provided. An analysis of variance (ANOVA) and subsequent post-hoc tests were carried out, and differences between samples were considered to be significant if $\mathrm{p} \leq 0.05$.

\subsubsection{Poisson's Ratio}

Poisson's ratio of the isotropic SIBS samples (Table 4) was assessed on the ELF tester following ASTM standard E 132-97. A camera was mounted and focused in front of the test specimens to allow imaging of each test sample at exactly the same location and focal setting. Longitudinal and transverse strain measurements were made as follows:

Four fiducial points $(0.2 \mathrm{~mm}$ in diameter $)$ were marked with India ink at the center of each specimen in symmetry with the horizontal and vertical axes, forming a rectangle. Once the sample was secured in the grips, prior to loading, an image was taken, showing the initial location of the points (Figure 13a). The specimen was then loaded until it reached $15 \%$ strain, and a second image was immediately taken, showing the final location of the points (Figure 13b). Care was taken to ensure that the second image occurred once the sample reached maximum elongation but before relaxation occurred. In addition to imaging the samples, a 1 x $1 \mathrm{~mm}$ grid was secured in the same location as the samples, and an image was taken. This image was used as a reference of known dimensions for quantification of Poisson's ratio.

In Photoshop ${ }^{\circledR}$, the image of the grid was overlaid on each of the samples images, and the locations of the four fiducial points were measured with reference to the grid at 0 
and 15\% strain (Figure 13). From this information, the horizontal and vertical distance between points was determined, and the Poisson's ratio was calculated as follows:

Equation 9. Poisson's ratio

$\mu=\frac{\varepsilon_{t}}{\varepsilon_{l}}$, where

$\mu$ : Poisson's ratio

$\varepsilon_{\mathrm{t}}:$ transverse strain

$\varepsilon_{1}:$ longitudinal strain.

Strain $(\varepsilon)$ was defined as:

Equation 10. Strain

$\varepsilon=\frac{l_{f}-l_{o}}{l_{o}}$, where

$1_{f}$ final length

$1_{0}$ : initial length.

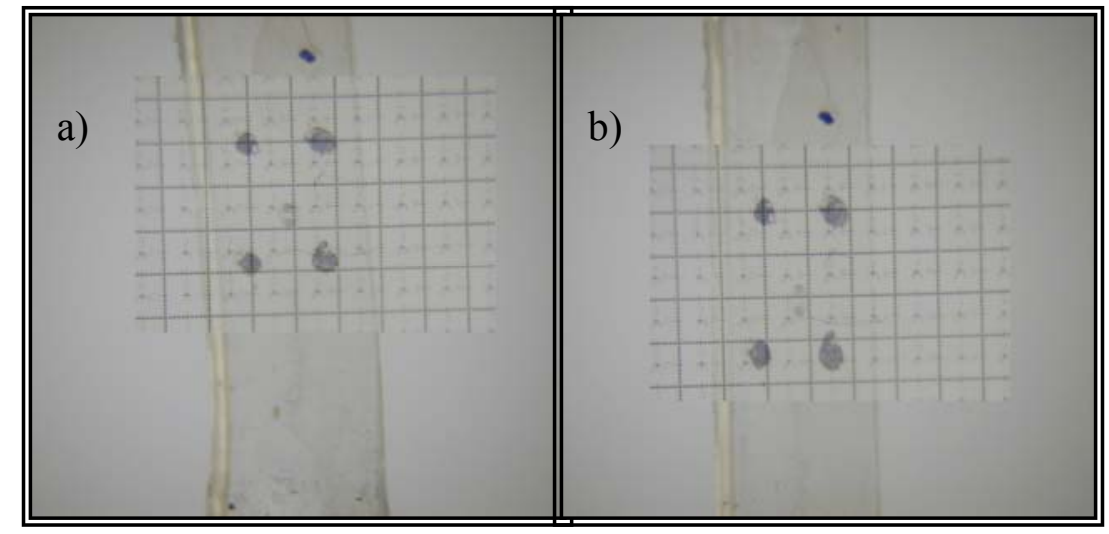

Figure 13. SIBS specimen (8.5\% styrene) mounted on the ELF tester showing the location of the four fiducial points at (a) $\varepsilon=0 \mathrm{~mm} / \mathrm{mm}$ and (b) $\varepsilon=0.15 \mathrm{~mm} / \mathrm{mm}$. A $1 \times 1 \mathrm{~mm}$ grid is shown overlaying the

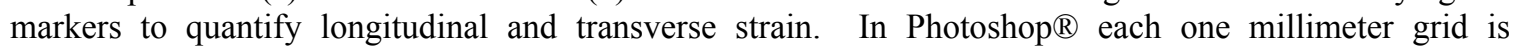
subdivided into 5 segments, providing a measurement precision of $0.2 \mathrm{~mm}$. A particularity of each point was used to identify its location at both strain locations. 
To reduce measurement errors, a particularity of each point was used as a measurement reference. At least five specimens were tested, taking into consideration that the standard deviation for each test was less than $10 \%$.

\subsubsection{Hydrodynamic Testing}

The hydrodynamic function of a prototype valve is key to assessing its potential for development. This method determines whether a valve is either stenotic or regurgitant. The hydrodynamic characteristics of the SIBS composite valves (listed in Table 5) were compared with two commercially available prosthetic valves: a St. Jude mechanical bileaflet valve and a Carpentier Edwards Magna pericardial prosthesis, both market leaders. The St. Jude mechanical bileaflet valve is the most common choice for heart valve replacement when a mechanical valve is the valve of choice, whereas the pericardial bioprosthesis is one of the valves of choice for bioprosthetic valves. The tissue annulus diameters for the SIBS and Magna valve were all $19 \mathrm{~mm}$ to allow a direct comparison between each; however, due to the lack of availability of a $19 \mathrm{~mm}$ mechanical valve, the St. Jude valve that was used had a $25 \mathrm{~mm}$ TAD.

The nine SIBS valve designs represent the various designs outlined in Section 4.1. The various stent designs incorporate different geometries and SIBS grades, which allow one to determine the impact of stent flexibility on valve hydrodynamics. By incorporating different leaflet manufacturing procedures and varying leaflet reinforcement fabrics, the orthotropic material properties of the leaflet can be manipulated, and their impact on valve hydrodynamics can be quantified. 
Table 5. Summary of valves subjected to hydrodynamic testing (refer to section 4.1 for the valve manufacturing procedure).

\begin{tabular}{|c|c|c|c|c|c|c|}
\hline \multirow[b]{3}{*}{ Valve } & \multicolumn{4}{|c|}{ Leaflet } & \multicolumn{2}{|c|}{ Stent } \\
\hline & \multirow[b]{2}{*}{ Design } & \multicolumn{2}{|c|}{ Reinforcement } & \multirow[b]{2}{*}{$\begin{array}{l}\text { Manufacturing } \\
\text { Technique }\end{array}$} & \multirow[b]{2}{*}{ Geometry } & \multirow[b]{2}{*}{$\begin{array}{l}\text { SIBS } \\
\text { Grade }\end{array}$} \\
\hline & & Type & \begin{tabular}{|c|} 
Orientation of \\
Max \\
Compliance \\
\end{tabular} & & & \\
\hline 1 & Design 1 & $\begin{array}{c}\text { PET Mesh } \\
1 \text { (LARS) }\end{array}$ & Circumferential & Dip coating & $\begin{array}{c}\text { Low } \\
\text { Profile }\end{array}$ & $\begin{array}{c}30 \% \\
\text { Styrene }\end{array}$ \\
\hline 2 & Design 2 & $\begin{array}{l}\text { PET Knit } \\
1 \text { (JSI) }\end{array}$ & Circumferential & Dip coating & $\begin{array}{c}\text { High } \\
\text { Profile }\end{array}$ & $\begin{array}{c}30 \% \\
\text { Styrene }\end{array}$ \\
\hline 3 & Design 2 & $\begin{array}{c}\text { PET Knit } \\
1 \text { (JSI) }\end{array}$ & Radial & Dip coating & $\begin{array}{c}\text { High } \\
\text { Profile }\end{array}$ & $\begin{array}{c}30 \% \\
\text { Styrene }\end{array}$ \\
\hline 4 & Design 3 & $\begin{array}{l}\text { PET Knit } \\
1 \text { (JSI) }\end{array}$ & Circumferential & Hybrid & $\begin{array}{l}\text { Medium } \\
\text { Profile }\end{array}$ & $\begin{array}{c}48.5 \% \\
\text { Styrene }\end{array}$ \\
\hline 5 & Design 4 & $\begin{array}{c}\text { PET Knit } \\
1 \text { (JSI) }\end{array}$ & Circumferential & Dip coating & $\begin{array}{l}\text { Medium } \\
\text { Profile }\end{array}$ & $\begin{array}{c}48.5 \% \\
\text { Styrene }\end{array}$ \\
\hline 6 & Design 4 & $\begin{array}{c}\text { PET } \\
\text { Weave }\end{array}$ & Circumferential & Solvent Casting & $\begin{array}{l}\text { Medium } \\
\text { Profile }\end{array}$ & $\begin{array}{c}48.5 \% \\
\text { Styrene }\end{array}$ \\
\hline 7 & Design 4 & $\begin{array}{l}\text { PET Mesh } \\
2 \text { (XA-47) }\end{array}$ & Circumferential & Solvent Casting & $\begin{array}{l}\text { Medium } \\
\text { Profile }\end{array}$ & $\begin{array}{c}48.5 \% \\
\text { Styrene }\end{array}$ \\
\hline 8 & Design 4 & $\begin{array}{l}\text { PET Mesh } \\
3 \text { (NZ-11) }\end{array}$ & Circumferential & Solvent Casting & $\begin{array}{l}\text { Medium } \\
\text { Profile }\end{array}$ & $\begin{array}{c}48.5 \% \\
\text { Styrene }\end{array}$ \\
\hline 9 & Design 4 & $\begin{array}{l}\text { PET Knit } \\
2 \text { (BARD) }\end{array}$ & Circumferential & Solvent Casting & $\begin{array}{l}\text { Medium } \\
\text { Profile }\end{array}$ & $\begin{array}{c}48.5 \% \\
\text { Styrene }\end{array}$ \\
\hline 10 & Magna & $\mathrm{N} / \mathrm{A}$ & N/A & N/A & $\mathrm{N} / \mathrm{A}$ & $\mathrm{N} / \mathrm{A}$ \\
\hline 11 & $\begin{array}{c}\text { St Jude } \\
\text { Bileaflet }\end{array}$ & N/A & N/A & N/A & N/A & N/A \\
\hline
\end{tabular}

\subsubsection{Vivitro Left Heart Simulator}

The valves were mounted in the aortic position of the Vivitro Systems (Vancouver, British Columbia) left heart and systemic circulation simulator (Figure 14). The system includes a processor-controlled stepper motor that drives a piston cylinder, resulting in contraction and relaxation of the left ventricular sac. The flow loop consists of a mitral and aortic valve mounted in anatomical positions, an aortic outflow track including a sinus of valsalva, and models of characteristic and peripheral resistance and 
compliance that allow the reproduction of systemic physiological flow and pressure waveforms (Figure 15).

Table 6. Specifications of cardiac outputs and heart rates for hydrodynamic testing of heart valves.

\begin{tabular}{|c|c|c|c|c|}
\hline Heart Rate & 45 & 70 & 100 & 120 \\
\hline Cardiac Output (L/min) & 3.6 & 5.6 & 8.0 & 9.6 \\
\hline
\end{tabular}

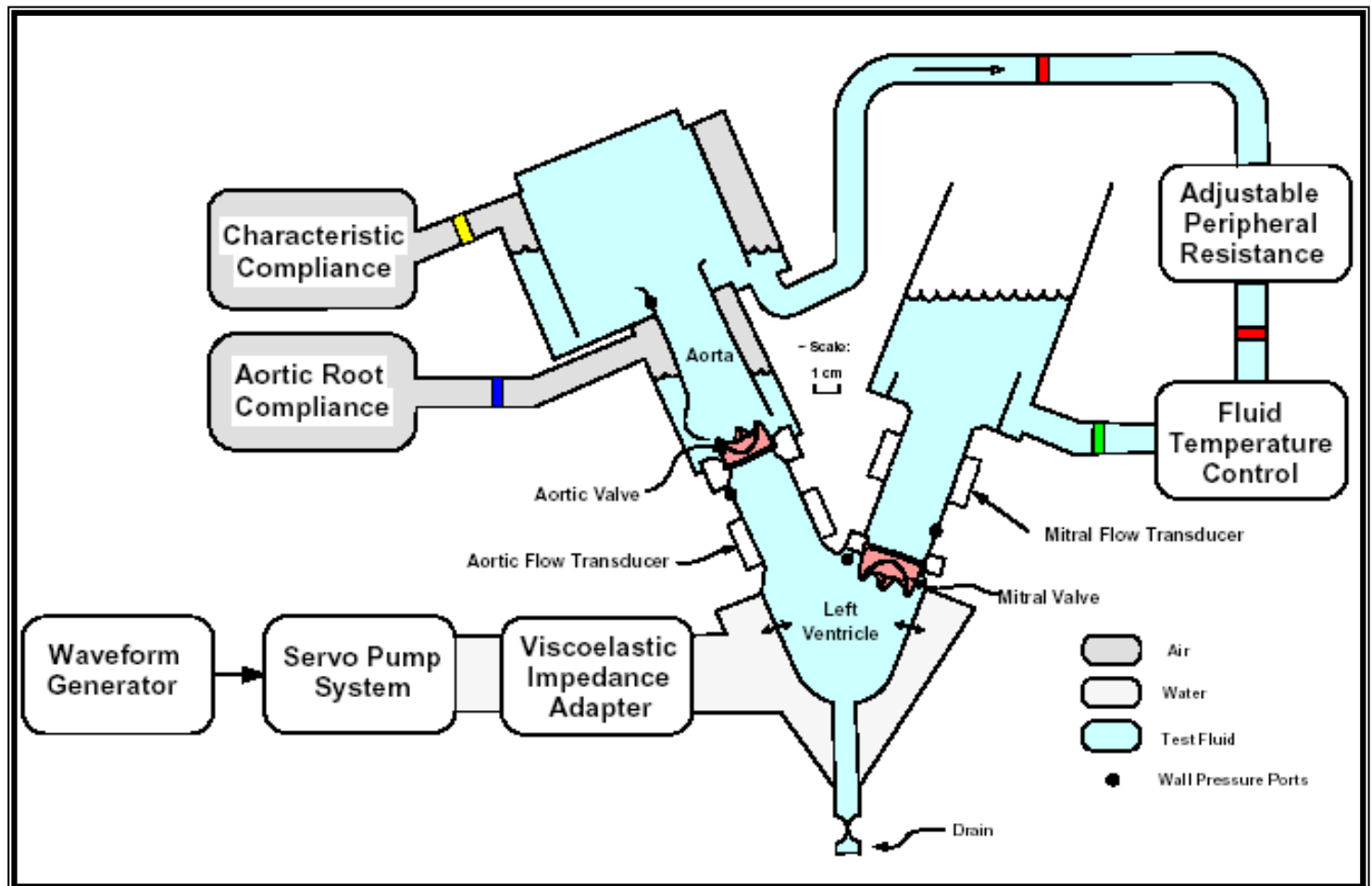

Figure 14. Schematic of the left heart and systemic circulation simulator: stroke volume $0-135 \mathrm{~mL}$, aortic root compliance air volume $0-585 \mathrm{~mL}$, characteristic compliance air volume $0-650 \mathrm{~mL}$, valve mounting up to size $33 \mathrm{~mm}$, blood analog fluid $2 \mathrm{~L}$

\subsubsection{2. $\quad$ Measurements and Outcome Measures}

Flow and pressure was measured for each of the valves at the combinations of cardiac outputs and heart rates presented in Table 6. Aortic flow was measured with an electromagnetic flow probe and meter (Carolina Medical Electronics, King, NC) that is 
mounted just below the aortic valve, as shown in Figure 14. Ventricular and aortic pressures were measured with catheter-tipped piezoelectric pressure transducers (Millar Instruments, Houston, TX), and data acquisition and storage was carried out with an MP100 data acquisition system and software (Biopac Systems, Inc., Santa Barbara, CA).

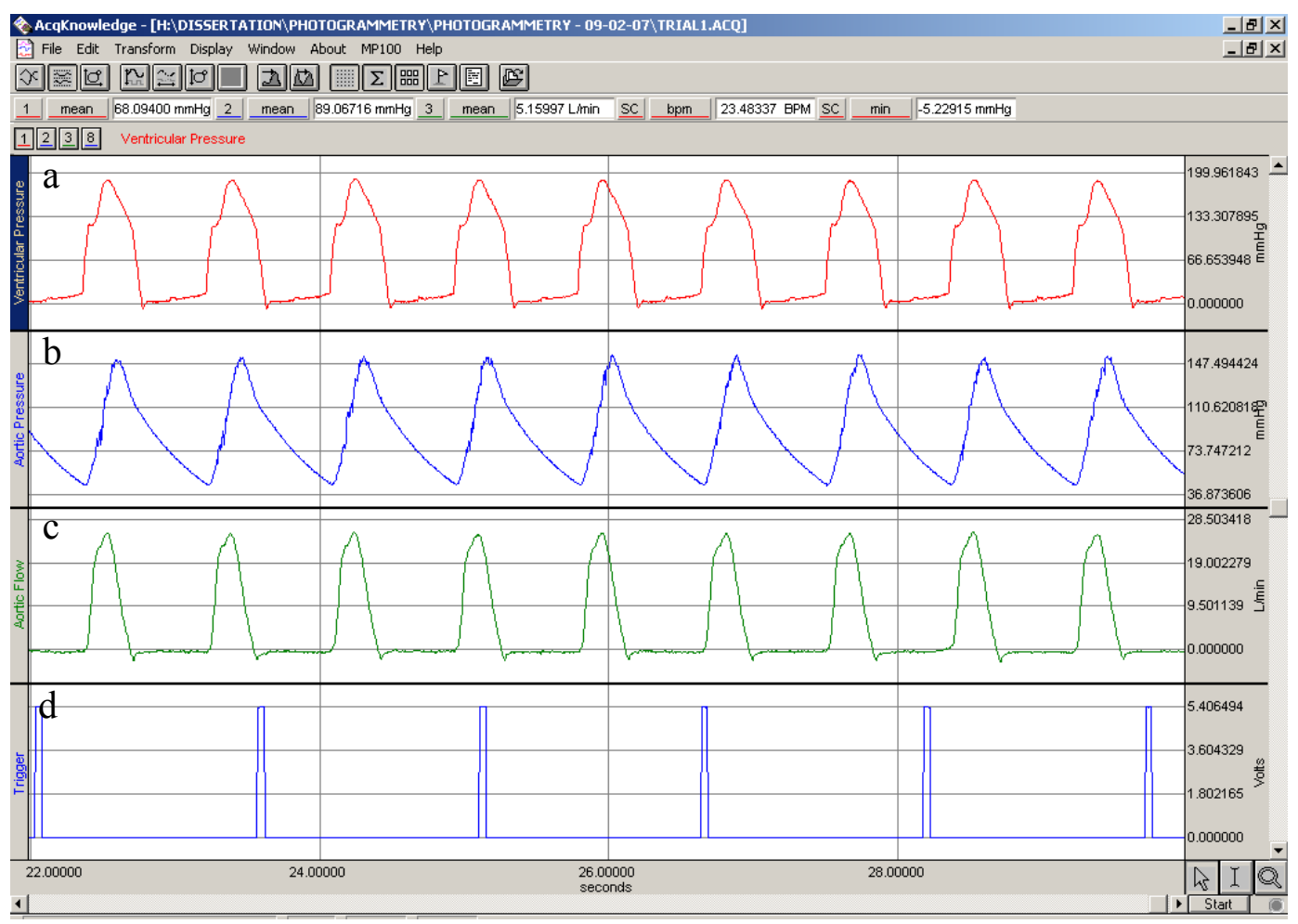

Figure 15. Typical pressure and flow waveforms showing (a) left ventricular pressure (mmHg), (b) aortic pressure $(\mathrm{mmHg}),(\mathrm{c})$ aortic flow $(\mathrm{L} / \mathrm{min})$, and camera trigger.

Flow and pressure was recorded for each valve at the respective cardiac output and heart rate combinations. Performance of the valves was assessed by measuring the mean transvalvular pressure drop during forward flow, the percent regurgitation during valve closure and while the valve was closed, and the effective orifice area (Equation 1). The values were averaged over 30 seconds of data acquisition, and three sets of measurements were taken for each valve and averaged. An ANOVA and subsequent 
post-hoc test was carried out, and any differences between each valve's performance was considered to be of significance if $\mathrm{p} \leq 0.05$.

As is evident from Figure 15, the pressure gradient across the valve is higher than what is expected for the natural valve; however, it is not high compared with other 19 mm TAD prosthetic valves. For example, Rosenhek et al. [76] reported the normal values of a variety of prosthetic valves through doppler echocardiographic measurements. For a $19 \mathrm{~mm}$ Bjork-Shiley tilting disk valve, the peak pressure gradient reported was 46.0 $\mathrm{mmHg}$ and the mean pressure gradient was $26.67 \mathrm{mmHg}$. For a Carpentier-Edwards stented bioprosthesis, the peak gradient was $43.48 \mathrm{mmHg}$, and the mean gradient was $25.6 \mathrm{mmHg}$. The $19 \mathrm{~mm}$ St. Jude Medical bileaflet valve was reported to have a peak gradient of $35.17 \mathrm{mmHg}$ and a mean gradient of $18.96 \mathrm{mmHg}$. The left heart and systemic circulation simulator cannot precisely match the natural system, but certain steps are taken to get the flow and pressure waves as close to the natural situation as possible. For example, a physiological flow curve is produced that replicates the expected cardiac outputs in vivo, and a mean aortic pressure of approximately $90-95$ $\mathrm{mmHg}$ is established. Not all left heart simulators can reproduce the same flow and pressure conditions. As a result, a comparative test must be carried out, where commercially available valves are tested on the same system for comparison.

\subsection{4. $S E M$}

SEM images of the valve were used to assess the quality of surface coverage, the quality of the free edge, and the presence of defects. Cyclic fatigue of the valve specimens can result in degradation of both the underlying fiber-reinforcement and the polymer itself. These are not always visible to the naked eye, even with the use of a 
microscope. SEM will be used to provide a more thorough understanding of the fatigue mechanisms of the fiber-reinforced SIBS valve.

The imaging technique is obviously a destructive one, so not all valves were made available for SEM analysis. For the fatigued valves that were analyzed, a pre-fatigue valve was necessary to compare the quality of the leaflet surface and leaflet free edge prior to any cycling. Particular attention was paid to surface smoothness and defects, such as cracks, holes, the presence of exposed reinforcement fibers, the presence of voids in cross-section, and the organization of reinforcement fibers (especially at the free edge).

It must be noted that any exposed reinforcement fabric has been proven to have a devastating impact on in vivo valve function, as will be discussed in the Preliminary Results, Section 5.1. For this reason, SEM can be used to evaluate the manufacturing process of the valve as well as how both the polymer and reinforcement fabric respond to cyclic fatigue.

\subsection{Specific Aim 2 - Finite Element Modeling}

Finite element modeling of the fiber reinforced heart valve was used to assess the affect of varying stent stiffness, fiber reinforcement, and leaflet geometry on the stress distributions in the leaflet. According to previous studies, each of these three parameters has a large impact on the stress concentrations in the leaflet. Vesely [21] reported that computational models have proven the advantage of flexible stent posts, a factor that has not been verified in vivo, and that the particular geometry of the coaptation region can have a large impact on leaflet stress concentrations, which was proven by a comparison of pericardial and porcine leaflets. Pericardial leaflets, that tend to have a horizontal 
coapting surface, have a higher concentration of stresses than do porcine leaflets. By applying incremental changes to the finite element model for the properties of stent flexibility, valve geometry, and reinforcement architecture, one can illustrate which properties result in a stress minimization, and a valve prototype can be developed for testing and verification of whether these model properties are feasible for the production of a valve with suitable function and durability. Ultimately, the objective was to find the best combination of these parameters in order to achieve a stress minimization, thereby increasing the potential for leaflet durability.

\subsubsection{Software}

Finite element modeling was carried out in ABAQUS (ABAQUS, Inc., Providence, RI), a simulation program capable of solving problems ranging from simple linear to complex nonlinear. ABAQUS is capable of modeling structural (stress/displacement) analyses, heat transfer, mass diffusion, and acoustics, to name a few.

All model geometry was designed in SolidWorks (SolidWorks Corporation, Concord, MA) and imported into ABAQUS/CAE (Complete ABAQUS Environment), where the complete model was created. ABAQUS/CAE is a graphical environment which allows the creation of geometry, material properties, loads, boundary conditions, and meshes. Once completed, the models can be submitted for analysis in either ABAQUS/Standard or ABAQUS/Explicit. ABAQUS/Standard uses the implicit method to solve a system of equations at each increment, whereas ABAQUS/Explicit utilizes the explicit method, which steps the solution through time. ABAQUS/Standard is a more general analysis tool that is capable of solving a variety of linear and nonlinear problems; 
ABAQUS/Explicit is more specialized, and it can model transient dynamic problems, including nonlinear problems that involve changing contact conditions. Once the analysis completes, results can be post-processed in ABAQUS/Viewer, a subset of ABAQUS/CAE.

\subsubsection{Model Description}

The models analyzed included those whose cylinder valve prototypes were tested in Aim 1 and additional theoretical models (refer to Table 7). In total there were eight models that were analyzed, which accounted for three different stent varieties, four different leaflet varieties, and three different leaflet coaptation geometries. The combination of the factors resulted in a total of four geometries, which are represented in

Figure 16 - Figure 19. A full factorial was not run, as a total of 36 models would be required to accomplish this. Instead, models were based on a selection of prototypes that were tested in both Aims 1 and 3, with the addition of four theoretical models that assessed the function of a non-reinforced leaflet, a valve mounted on a completely rigid stent, and various coaptation geometries.

A schematic of the general valve prototype is provided in Figure 20, showing the locations of the leaflet commissure, coaptation, and belly; the stent; and the leaflet-stent junction. Each valve consists of three identical leaflets that are securely attached to the supporting stent. Each leaflet has a uniform thickness distribution that is defined by the particular reinforcement fabric and fabrication technique for the specific model. Although each third of the valve (including an individual leaflet and its corresponding stent third) is symmetrical and each individual leaflet has a symmetry plane, conditions of symmetry were not taken advantage of. Instead, it was believed to be more beneficial to 
model the valve as a whole in order to accurately represent the contact interactions between each coapting leaflet and to accurately represent the response of the stent to loading.

Table 7. Summary of valve models. Refer to section 4.1 for the valve models that represent experimental prototypes, and refer to Figure 16 - Figure 19 for the specific valve geometries.

\begin{tabular}{|c|c|c|c|c|c|c|}
\hline \multirow{3}{*}{ Valve } & \multicolumn{4}{|c|}{ Leaflet } & \multicolumn{2}{|c|}{ Stent } \\
\hline & \multicolumn{2}{|c|}{ Reinforcement } & \multirow[b]{2}{*}{$\begin{array}{l}\text { Manufacturing } \\
\text { Technique }\end{array}$} & \multirow[b]{2}{*}{$\begin{array}{l}\text { Coaptation } \\
\text { Geometry }\end{array}$} & \multirow[b]{2}{*}{ Geometry } & \multirow[b]{2}{*}{$\begin{array}{l}\text { SIBS } \\
\text { Grade }\end{array}$} \\
\hline & Type & $\begin{array}{c}\text { Orientation of } \\
\text { Max } \\
\text { Compliance } \\
\end{array}$ & & & & \\
\hline 1 & $\begin{array}{l}\text { PET Knit } \\
1 \text { (JSI) }\end{array}$ & Circumferential & $\begin{array}{l}\text { Design } 2 \\
8.5 \% \text { SIBS }\end{array}$ & $\begin{array}{l}\text { Slight } \\
\text { Curve }\end{array}$ & $\begin{array}{l}\text { High } \\
\text { Profile }\end{array}$ & $\begin{array}{c}30 \% \\
\text { Styrene }\end{array}$ \\
\hline 2 & $\begin{array}{l}\text { PET Knit } \\
1 \text { (JSI) }\end{array}$ & Radial & $\begin{array}{c}\text { Design } 2 \\
8.5 \% \text { SIBS }\end{array}$ & $\begin{array}{l}\text { Slight } \\
\text { Curve }\end{array}$ & $\begin{array}{l}\text { High } \\
\text { Profile }\end{array}$ & $\begin{array}{c}30 \% \\
\text { Styrene }\end{array}$ \\
\hline 3 & $\begin{array}{l}\text { PET Knit } \\
1 \text { (JSI) }\end{array}$ & Circumferential & $\begin{array}{l}\text { Design } 4 \\
8.5 \% \text { SIBS }\end{array}$ & $\begin{array}{l}\text { Slight } \\
\text { Curve }\end{array}$ & $\begin{array}{l}\text { Medium } \\
\text { Profile }\end{array}$ & $\begin{array}{l}48.5 \% \\
\text { Styrene }\end{array}$ \\
\hline 4 & None & N/A & $\begin{array}{c}\text { Theoretical } \\
\text { Isotropic } 8.5 \% \\
\text { SIBS }\end{array}$ & $\begin{array}{l}\text { Slight } \\
\text { Curve }\end{array}$ & $\begin{array}{l}\text { Medium } \\
\text { Profile }\end{array}$ & $\begin{array}{l}48.5 \% \\
\text { Styrene }\end{array}$ \\
\hline 5 & $\begin{array}{c}\text { PET Knit } \\
2 \\
\text { (BARD) } \\
\end{array}$ & Circumferential & Design 4 & $\begin{array}{l}\text { Slight } \\
\text { Curve }\end{array}$ & $\begin{array}{l}\text { Medium } \\
\text { Profile }\end{array}$ & $\begin{array}{c}\text { Theoretical, } \\
\text { Rigid }\end{array}$ \\
\hline 6 & $\begin{array}{c}\text { PET Knit } \\
2 \\
\text { (BARD) } \\
\end{array}$ & Circumferential & Design 4 & $\begin{array}{l}\text { Slight } \\
\text { Curve }\end{array}$ & $\begin{array}{l}\text { Medium } \\
\text { Profile }\end{array}$ & $\begin{array}{l}48.5 \% \\
\text { Styrene }\end{array}$ \\
\hline 7 & $\begin{array}{c}\text { PET Knit } \\
2 \\
\text { (BARD) }\end{array}$ & Circumferential & Design 4 & $\begin{array}{c}\text { Theoretical, } \\
\text { Flat }\end{array}$ & $\begin{array}{l}\text { Medium } \\
\text { Profile }\end{array}$ & $\begin{array}{l}48.5 \% \\
\text { Styrene }\end{array}$ \\
\hline 8 & $\begin{array}{c}\text { PET Knit } \\
2 \\
\text { (BARD) }\end{array}$ & Circumferential & Design 4 & $\begin{array}{c}\text { Theoretical, } \\
\text { Larger } \\
\text { Curve }\end{array}$ & $\begin{array}{l}\text { Medium } \\
\text { Profile }\end{array}$ & $\begin{array}{l}48.5 \% \\
\text { Styrene }\end{array}$ \\
\hline
\end{tabular}

Previously, Liu et al. [44] modeled the SIBS valve leaflet as a laminated composite consisting of three layers: two isotropic, homogeneous outer layers and an inner fiber-reinforced composite. In Liu's model, the SIBS leaflet was reinforced with individual polypropylene fibers. In order to define the material properties, the individual properties of the SIBS and a polypropylene fiber were required. The homogeneous, 
isotropic properties of SIBS were used to define the homogeneous outer layers, whereas the Halpin-Tsai model was adopted to define the properties of the polypropylenereinforced inner layer by applying the properties of SIBS, polypropylene, and the volume percentage of polypropylene. This method of establishing the material properties is not feasible for the particular model under investigation now because the reinforcement materials utilized are significantly more complex. Commercially available multifilament polyester knits were used to reinforce the valve (refer to Table 7). With these types of fabrics, fibers interact with each other, and when they are coated with SIBS, this interaction property changes. As a result, the Halpin-Tsai model cannot predict the material properties of the fiber-reinforced layer from the knowledge of the individual properties of the knit and polymer. Due to manufacturing constraints, it was impossible to separate the homogeneous outer layers of SIBS from the inner fiber reinforced layer for testing, so the material properties were determined, according to Sections 4.2.1 and 4.2.2, for the full thickness of the material and input into the model. For this reason, the leaflet was modeled as a homogeneous orthotropic shell instead of as a composite shell.

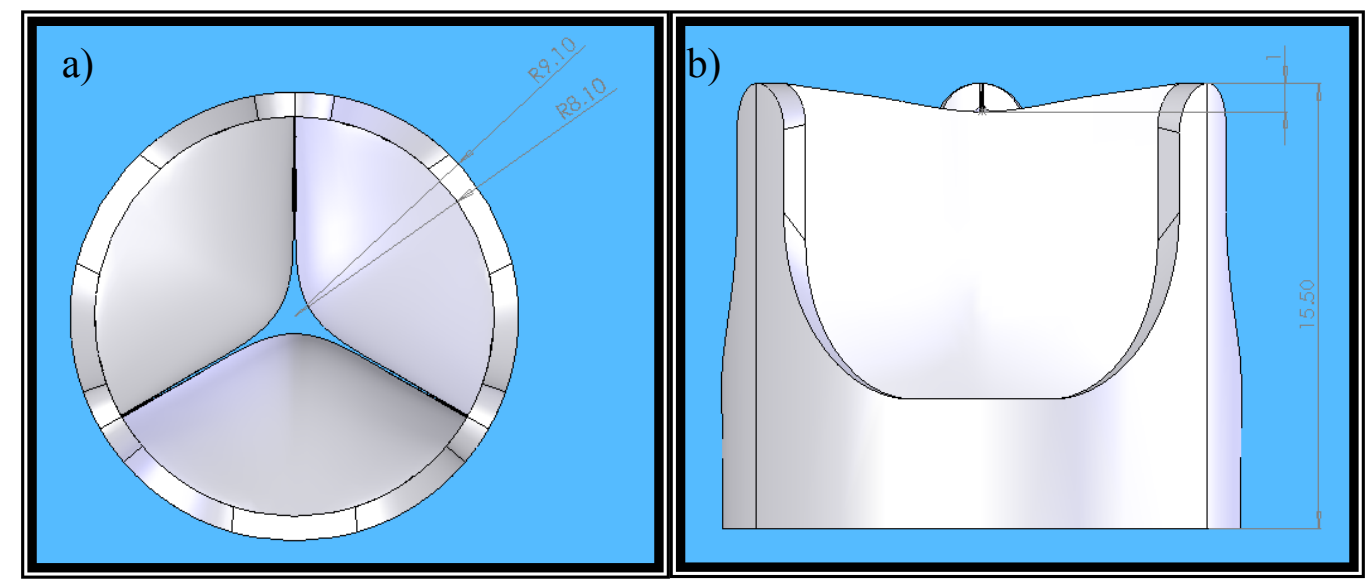

Figure 16. Geometry for models showing the a) top view and b) side view of the high profile stent with "slight curve" leaflet curvature. 


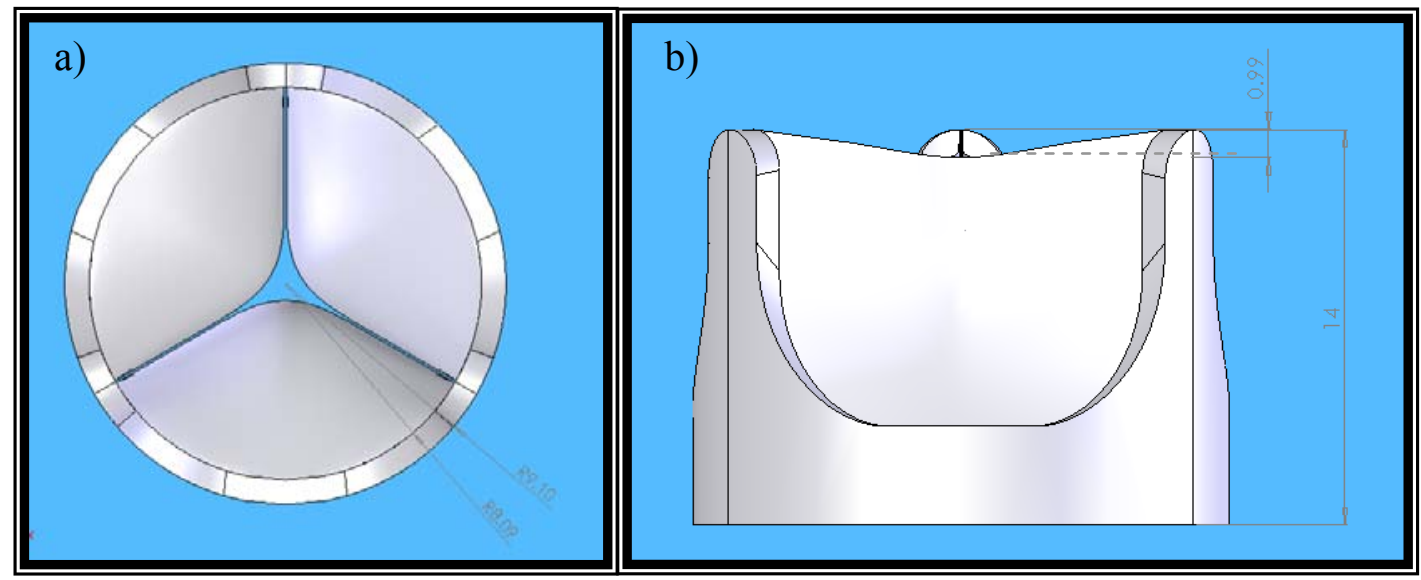

Figure 17. Geometry for models showing the a) top view and b) side view of the medium profile stent with "slight curve" leaflet curvature.

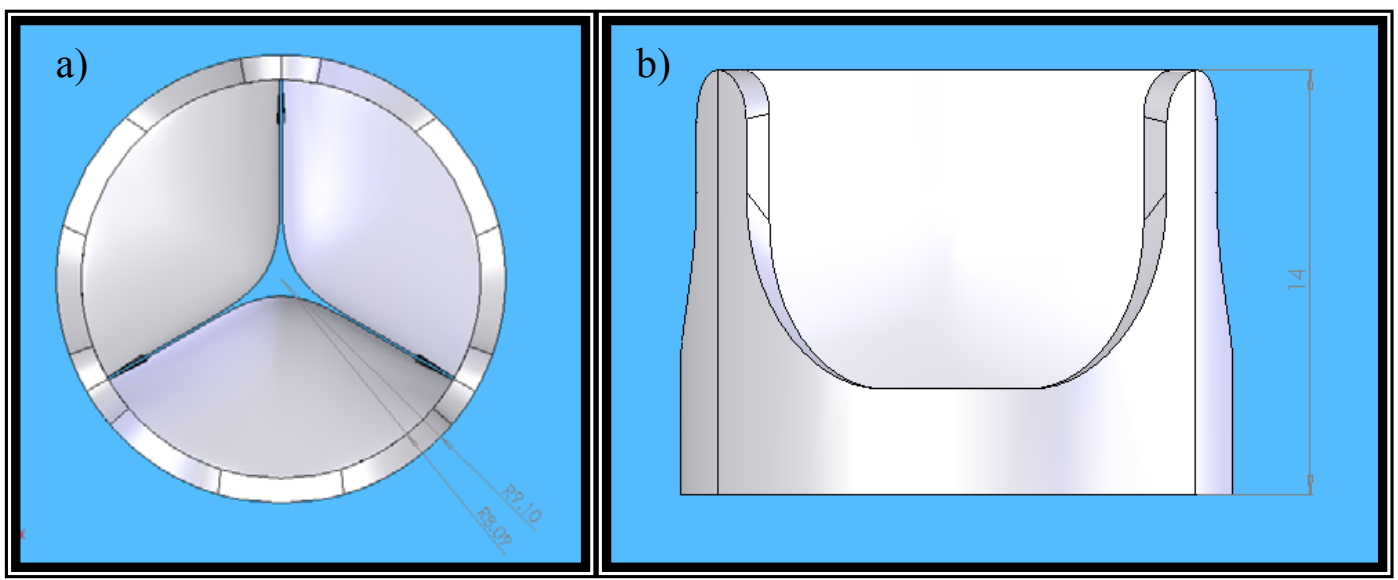

Figure 18. Geometry for models showing the a) top view and b) side view of the medium profile stent with "flat" leaflet curvature.

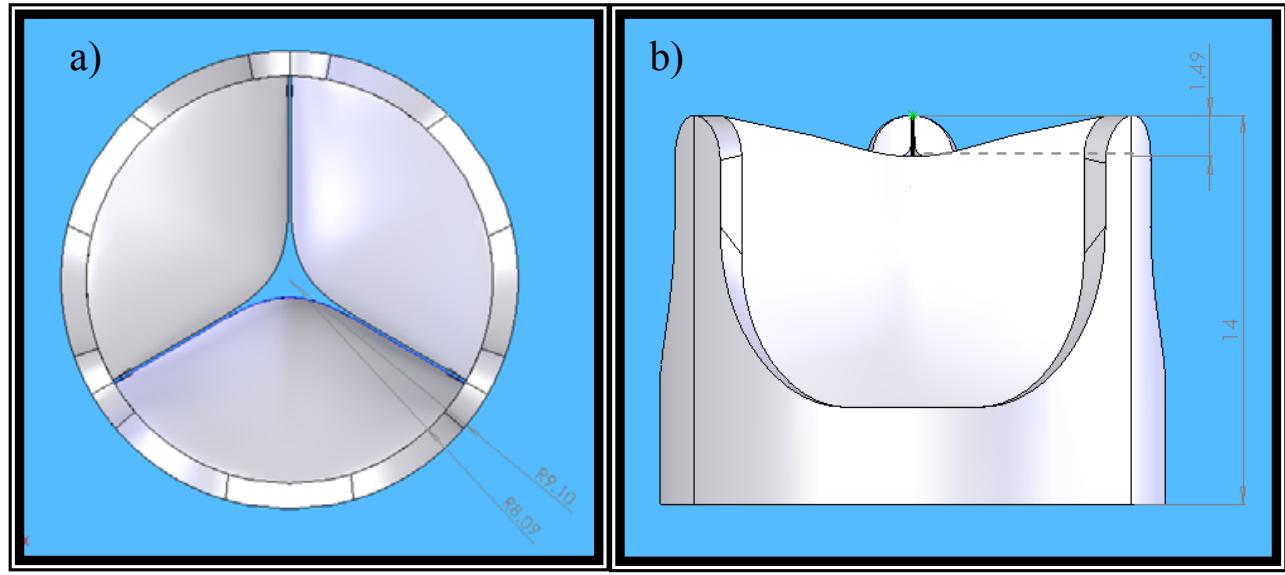

Figure 19. Geometry for models showing the a) top view and b) side view of the medium profile stent with "larger curve" leaflet curvature. 


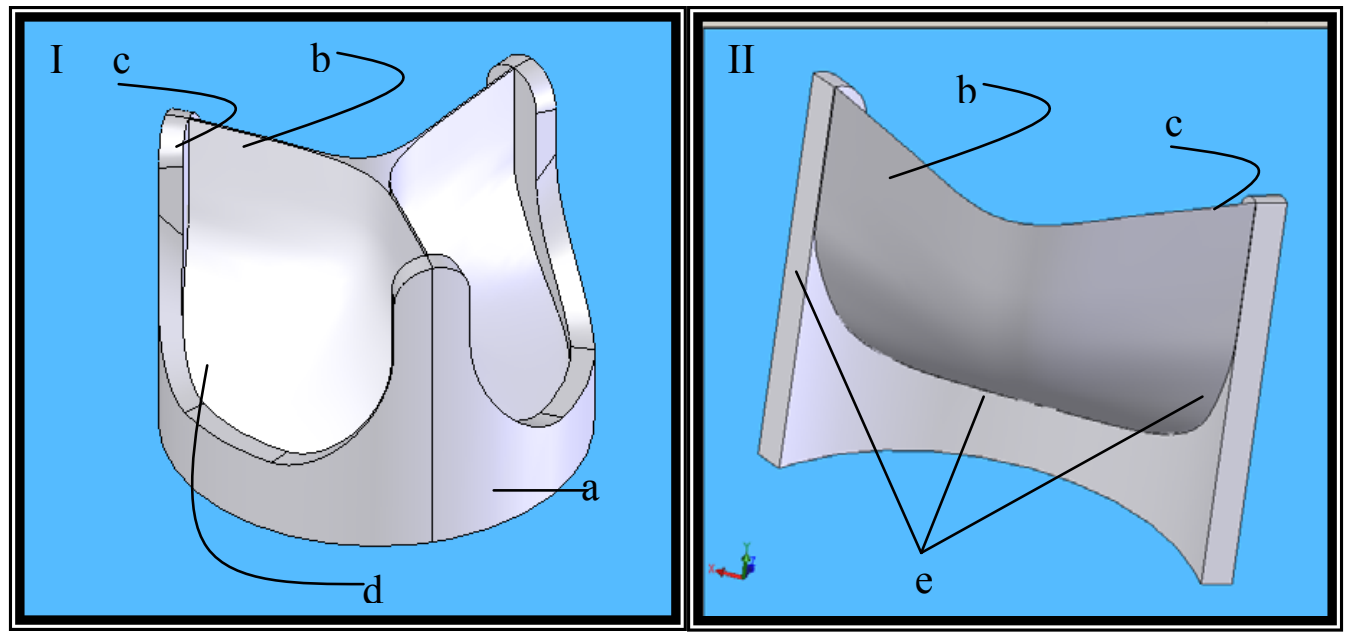

Figure 20. Schematic of the (I) whole polymer trileaflet valve and (II) one third of the valve with interior detail. Labeled segments represent the (a) stent, (b) area of coaptation, (c) commissure, (d) leaflet belly, e) stent-leaflet joint. Embedded in the polymer is a reinforcement fabric.

\subsubsection{Boundary Conditions}

Generation of the geometrical model in SolidWorks was accomplished by designing one third of the valve, including the leaflet and attached stent, and creating a circular pattern to define the remaining two thirds. In total, the model consisted of six individual parts (three leaflets and three stents), and the interactions between each component had to be specified. ABAQUS does not recognize contact by the mere proximity of parts to one another, as a result, each individual contact and attachment constraint had to be specified by means of either a contact or tie condition, respectively. A summary of the boundary conditions is provided in Table 8, and a description of each condition is given below.

Tie constraints can be used to tie two surfaces or node sets together so that each node on the slave surface is constrained to have the same motion as the node on the master surface that is in closest proximity. This means that the translational and rotational degrees of freedom are constrained. Tie constraints can effectively be used to 
model the attachment regions between adjacent stent thirds and between the stent and attached leaflet, thereby ensuring forces acting on the leaflet are transferred to the stent, and ensuring that the stent acts as one piece instead of individual thirds. In the SIBS valve model, six tie constraints were defined in total: three to model the attachment between each leaflet and stent section (Figure 21a), and three to model the stent-to-stent connections along each individual post (Figure 21b).

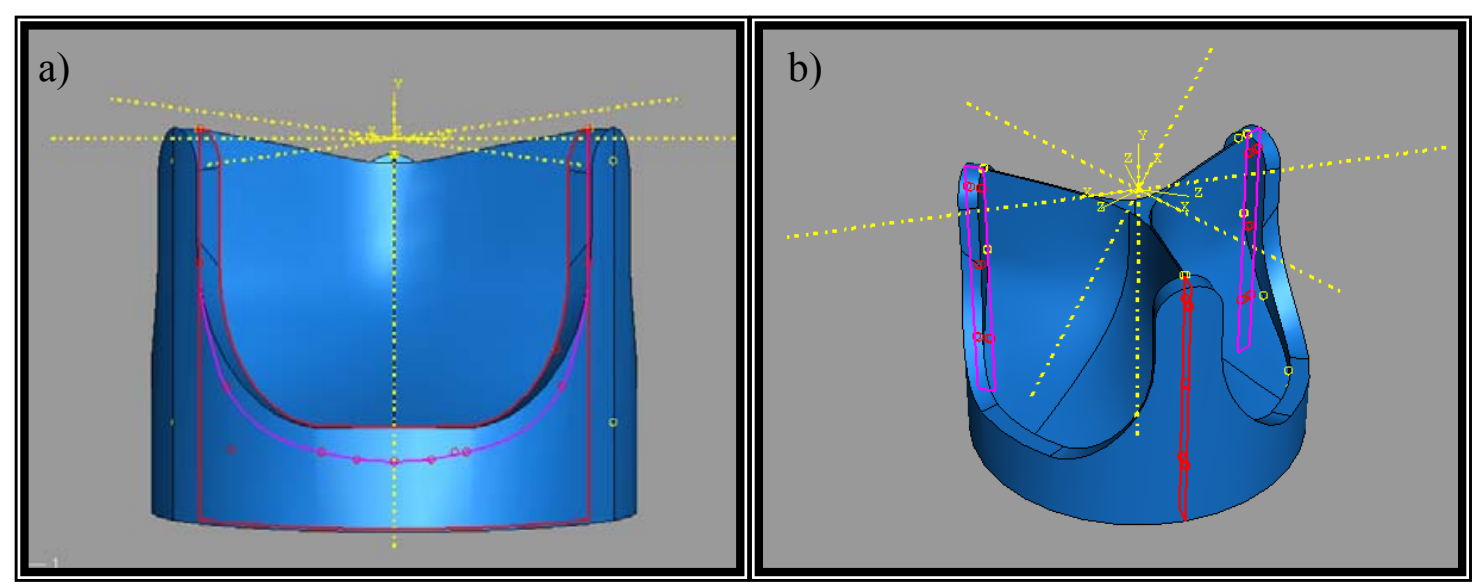

Figure 21. Image showing the contact pairs between (a) a leaflet and its corresponding stent and (b) adjacent stents. The tie constraint establishes a bond between adjacent stent thirds and between the contacting leaflet and stent sections. This bond ensures that the nodes in closest proximity are restricted to move as one.

In the SIBS valve model, contact interactions come into play during both diastole and systole. During diastole the maximum pressure occurs on the aortic side of the leaflet, forcing the valve to close and the adjacent leaflets to coapt (Figure 22a). During systole, the maximum pressure occurs on the ventricular side of the leaflets, forcing the leaflets to open. When open, the leaflets are restricted from deforming past the stent, so a contact interaction develops between each corresponding leaflet and stent section (Figure 22b). In order to model contact in ABAQUS, the contact pairs and the constitutive 
models governing the contact interactions had to be defined. The finite-sliding contact formulation was instituted in the SIBS valve model as this formulation allows a continuous update during the simulation for the definition of which part of the master surface is in contact with the slave node. The tangential frictional behavior between surfaces was defined according to the Penalty friction formulation, and a friction coefficient of 0.3 was selected as recommended by Sun et al. [62]. Sun et al. simulated the quasi-static loading of a bioprosthetic heart valve and experimented with friction coefficients ranging from 0.0 to 0.5 . As the friction coefficient was increased from 0.0 to 0.3 , an increase in the peak strain in the leaflet was observed; however, minimal increase in the peak strain was witnessed when the friction coefficient was increased from 0.3 to 0.5. As a result, the optimal friction coefficient was chosen to be 0.3 . The Penalty formulation was selected due to its ease of implementation for multiple contact formulations. This method allows a small amount of elastic slip between contacting surfaces, and this slip is automatically calculated to be a small fraction of the characteristic element length.

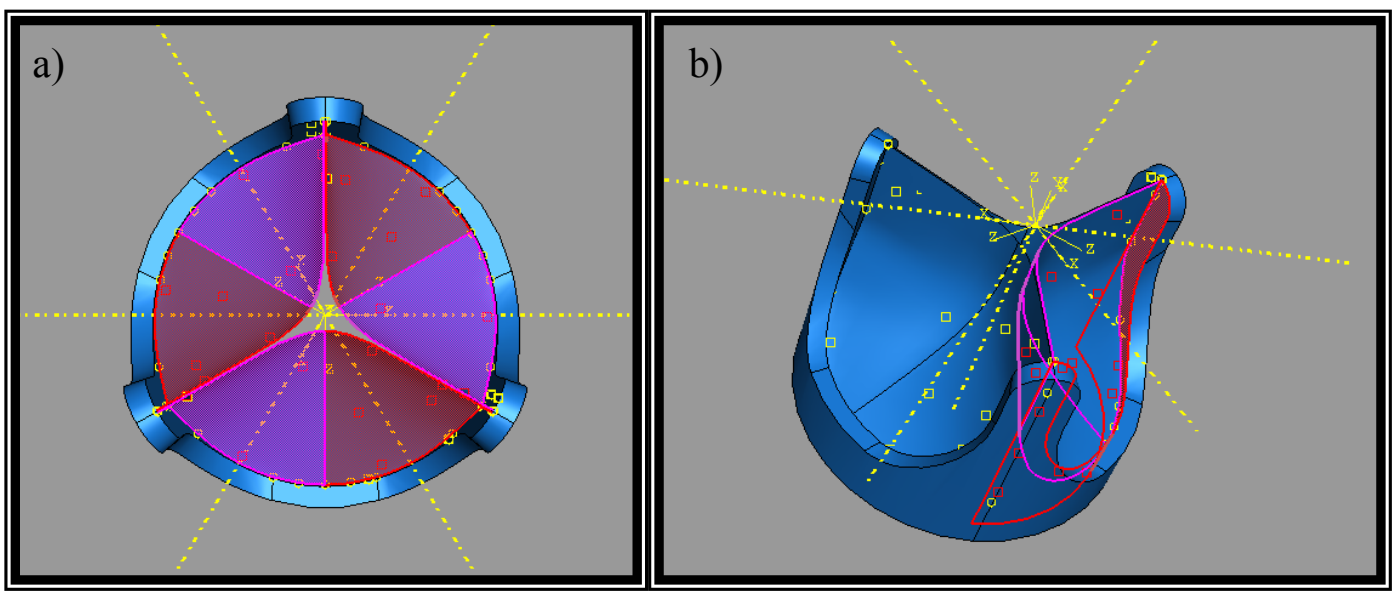

Figure 22. Image showing the contact interaction pairs between a) coapting leaflet segments and b) leafletstent contacting regions during systole. The contact constraint alows the definition of the tangential frictional behavior between two contacting surfaces. 


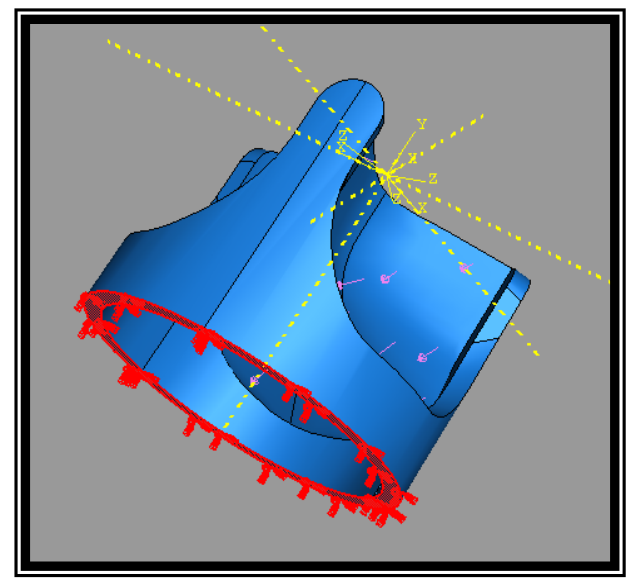

Figure 23. Image showing the fixed boundary condition applied to the base of the stent. A fixed boundary condition means that that base of the stent is restricted from either translating or rotating, making it a completely rigid restraint.

Table 8. A summary of the boundary conditions for all valve models. A total of 13 boundary conditions was instituted.

\begin{tabular}{|l|l|l||}
\hline \hline Type & Location & Description \\
\hline Tie Constraint & $\begin{array}{l}\text { Adjacent stent } \\
\text { thirds (3 in } \\
\text { total) }\end{array}$ & $\begin{array}{l}\text { This boundary condition constrains adjacent stent thirds so that } \\
\text { they behave as one. At the contact surface, nodes on the slave } \\
\text { surface are constrained to move in the same manner as nodes on } \\
\text { the master surface. }\end{array}$ \\
\hline Tie Constraint & $\begin{array}{l}\text { Contact point } \\
\text { between leaflet } \\
\text { and adjacent } \\
\text { stent segment } \\
\text { (3 in total) }\end{array}$ & $\begin{array}{l}\text { This boundary condition constrains the contact interaction } \\
\text { between the nodes on the leaflet edge and the nodes on the } \\
\text { surface of the contacting stent segment. The nodes on the } \\
\text { leaflet edge are constrained to move in the same manner as } \\
\text { nodes on the stent surface. }\end{array}$ \\
\hline $\begin{array}{l}\text { Contact } \\
\text { Constraint } \\
\text { leaflets (3 in } \\
\text { total) }\end{array}$ & $\begin{array}{l}\text { This contact constraint simulates leaflet coaptation during } \\
\text { diastole. Adjacent leaflets are restricted from moving past each } \\
\text { other and tangential friction effects are specified to allow } \\
\text { accurate simulation of contact. }\end{array}$ \\
\hline $\begin{array}{l}\text { Contact } \\
\text { Constraint }\end{array}$ & $\begin{array}{l}\text { Leaflet and } \\
\text { adjacent stent } \\
\text { segment } 3 \text { in } \\
\text { total) }\end{array}$ & $\begin{array}{l}\text { This contact constraint prevents the leaflet from moving } \\
\text { outward past the supporting stent, and once again tangential } \\
\text { friction effects are specified to allow accurate simulation of } \\
\text { contact. }\end{array}$ \\
\hline $\begin{array}{l}\text { Fixed Boundary } \\
\text { Condition }\end{array}$ & $\begin{array}{l}\text { Stent base (1 } \\
\text { in total) }\end{array}$ & $\begin{array}{l}\text { This boundary condition allows the simulation of valve restraint } \\
\text { either in vitro or in vivo. }\end{array}$ \\
\hline
\end{tabular}

During both in vitro testing and in vivo implantation of the SIBS valve, the valve

is secured in place by an attachment at the stent base. In vitro, a polycarbonate ring is

bonded to the stent base, and this is secured in a silicone ring for placement in either the

Vivitro hydrodynamic or fatigue tester; in vivo, a suture cuff is secured to the base of the 
stent, and this is sutured into the aorta. As a result, a fixed boundary condition was applied to the base of the stent to simulate its attachment criteria (Figure 23).

\subsubsection{Material Properties}

The material properties required for input into the FEM included the Young's modulus (E), the Poisson's ratio (v), and the shear modulus (G). The tensile and Poisson's ratio properties for both the leaflets and stent were determined according to the procedures in Sections 4.2.1 and 4.2.2 and their resultant values are presented in Section 5. The specifics for each material model are discussed in detail below.

The stent is fabricated from homogeneously isotropic compression molded SIBS of either 30 or $48.5 \%$ styrene content. At the strain level experienced by the stent, these materials can be considered to be linearly elastic. For linearly elastic materials, the properties can be completely defined by E and $v$, and the shear modulus can be calculated as follows:

Equation 11. Shear modulus

$$
G=\frac{E}{2(1+v)}
$$

The stress-strain relationship for the material is then given by:

Equation 12. Stress-Strain relationship for a linearly elastic isotropic material.

$$
\left\{\begin{array}{l}
\varepsilon_{11} \\
\varepsilon_{22} \\
\varepsilon_{33} \\
\gamma_{12} \\
\gamma_{13} \\
\gamma_{23}
\end{array}\right\}=\left[\begin{array}{cccccc}
1 / \mathrm{E} & -v / \mathrm{E} & -v / \mathrm{E} & 0 & 0 & 0 \\
-v / \mathrm{E} & 1 / \mathrm{E} & -v / \mathrm{E} & 0 & 0 & 0 \\
-v / \mathrm{E} & -v / \mathrm{E} & 1 / \mathrm{E} & 0 & 0 & 0 \\
0 & 0 & 0 & 1 / \mathrm{G} & 0 & 0 \\
0 & 0 & 0 & 0 & 1 / \mathrm{G} & 0 \\
0 & 0 & 0 & 0 & 0 & 1 / \mathrm{G}
\end{array}\right]\left\{\begin{array}{l}
\sigma_{11} \\
\sigma_{22} \\
\sigma_{33} \\
\sigma_{12} \\
\sigma_{13} \\
\sigma_{23}
\end{array}\right\}
$$


ABAQUS simply requires $\mathrm{E}$ and $v$ as input, and all other values specified in Equation 11 and Equation 12 are calculated automatically. For the theoretical, rigid stent (Table 7), the stent was also modeled as an isotropic, linearly elastic material instead of as a rigid body. The main reason for this was ease of implementation. Models already existed with the material definitions stated above, so it was easier to change the mechanical properties than to institute a rigid body definition. In order to ensure the stent did perform as a rigid body, the Young's modulus input was 10,000 times greater than the highest modulus input for any other model.

The leaflet materials that were modeled included a polyester knit (Either JSI or BARD) that was either dip-coated or solvent cast with an $8.5 \%$ styrene SIBS. When instituted as a valve leaflet, the primary directions of these orthotropic materials are oriented in the circumferential and radial directions. The leaflet material response can be considered to be linearly elastic at the anticipated strain level, so the leaflet could be modeled as a linearly elastic orthotropic material. In order to define the material properties, nine engineering constants were required, namely $E_{1}, E_{2}, E_{3}, v_{12}, v_{13}, v_{23}, G_{12}$, $\mathrm{G}_{13}$, and $\mathrm{G}_{23}$. In the case of the valve leaflet, the 1-2-, and 3-directions were considered to be the circumferential, radial, and through thickness directions respectively. The Young's moduli and Poisson's ratio for the radial and circumferential directions were determined by the tests described in Section 4.2.1 and 4.2.2, but due to the fact that the through thickness properties could not be measured directly, the values were assumed to be that of pure $8.5 \%$ SIBS. The shear modulus was defined according to Equation 11, and the stress-strain relationship is given by: 
Equation 13. Stress-strain relationship for orthotropic elasticity.

$$
\left\{\begin{array}{l}
\varepsilon_{11} \\
\varepsilon_{22} \\
\varepsilon_{33} \\
\gamma_{12} \\
\gamma_{13} \\
\gamma_{23}
\end{array}\right\}=\left[\begin{array}{cccccc}
1 / \mathrm{E}_{1} & -v_{21} / \mathrm{E}_{2} & -v_{31} / \mathrm{E}_{3} & 0 & 0 & 0 \\
-v_{12} / \mathrm{E} & 1 / \mathrm{E}_{2} & -v_{32} / \mathrm{E}_{3} & 0 & 0 & 0 \\
-v_{13} / \mathrm{E}_{1} & -v_{23} / \mathrm{E}_{2} & 1 / \mathrm{E}_{3} & 0 & 0 & 0 \\
0 & 0 & 0 & 1 / \mathrm{G}_{12} & 0 & 0 \\
0 & 0 & 0 & 0 & 1 / \mathrm{G}_{13} & 0 \\
0 & 0 & 0 & 0 & 0 & 1 / \mathrm{G}_{23}
\end{array}\right]\left\{\begin{array}{l}
\sigma_{11} \\
\sigma_{22} \\
\sigma_{33} \\
\sigma_{12} \\
\sigma_{13} \\
\sigma_{23}
\end{array}\right\}
$$

For the model evaluating the isotropic SIBS leaflets, the material was considered to be isotropic elastic, so the material was defined as outlined for the stent.

\subsubsection{Material Orientation and Coordinate System}

The valve was defined according to a rectangular coordinate system, and the origin of the global coordinate system was located at the exact center of the valve at its highest point (Figure 24). For isotropic material components, directionality has no impact on the material properties, so no material orientation is required. By default, the 1-, 2-, and 3- directions refer to the global coordinate system. For orthotropic materials, however, the material properties are highly dependent on their orientation, so it became necessary to assign a local coordinate system for each individual leaflet and orient the material properties according to that system. With this method, it was possible to orient the circumferential direction along local direction 1 , the radial direction along local direction 2, and the through thickness or normal direction along local direction 3 (Figure 25). This allowed each leaflet to be defined according to their measured circumferential, radial, and through thickness properties. 


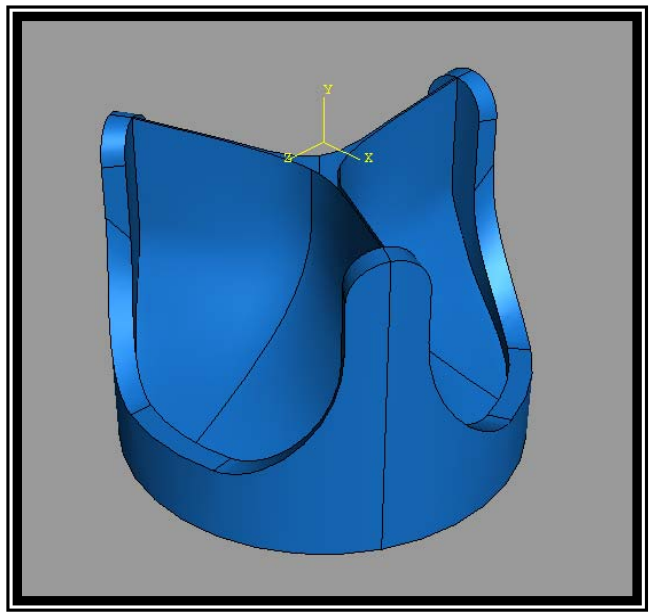

Figure 24. Model of valve showing the global coordinate system location at the highest central point of the valve.

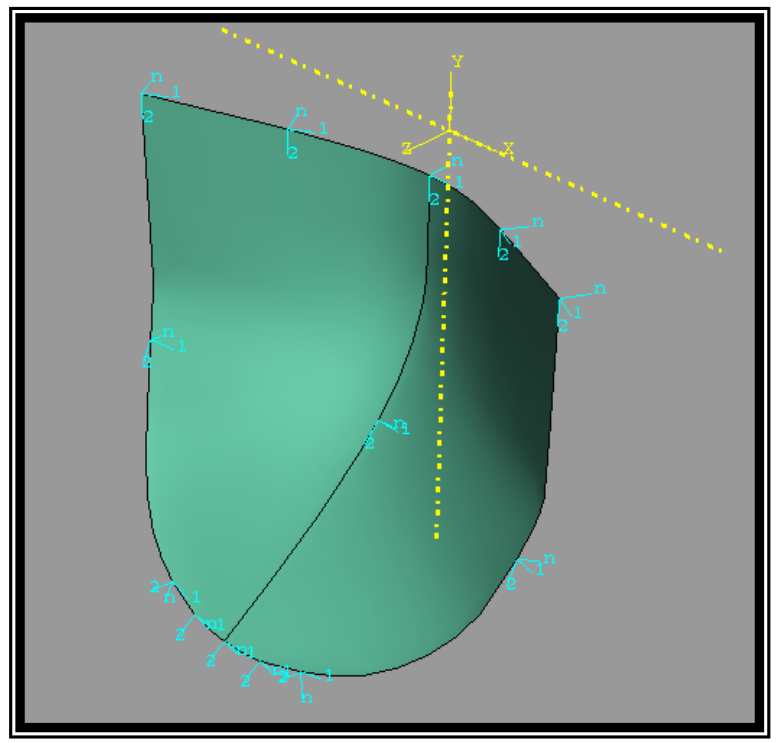

Figure 25. Individual leaflet showing the location of the local coordinate system and the orientation of the $1-, 2-$, and 3(n)-directions.

\subsubsection{Loading Conditions}

A typical pressure waveform showing the ventricular, aortic, and transvalvular pressures obtained during the hydrodynamic testing of a SIBS valve is shown in Figure 26. From this figure, it is apparent that the pressure exerted on the valve is highly variable over time, and that the maximum transvalvular pressure acting on the valve 
occurs during diastole, while the valve is closed. With this knowledge in mind, a quasistatic analysis procedure for leaflet stress evaluation was carried out. The valve was loaded from a zero pressure state to the maximum diastolic load, where the transvalvular pressure load obtained from the hydrodynamic evaluation of the valve was applied to the aortic surface of the leaflets, resulting in a uniform pressure distribution.

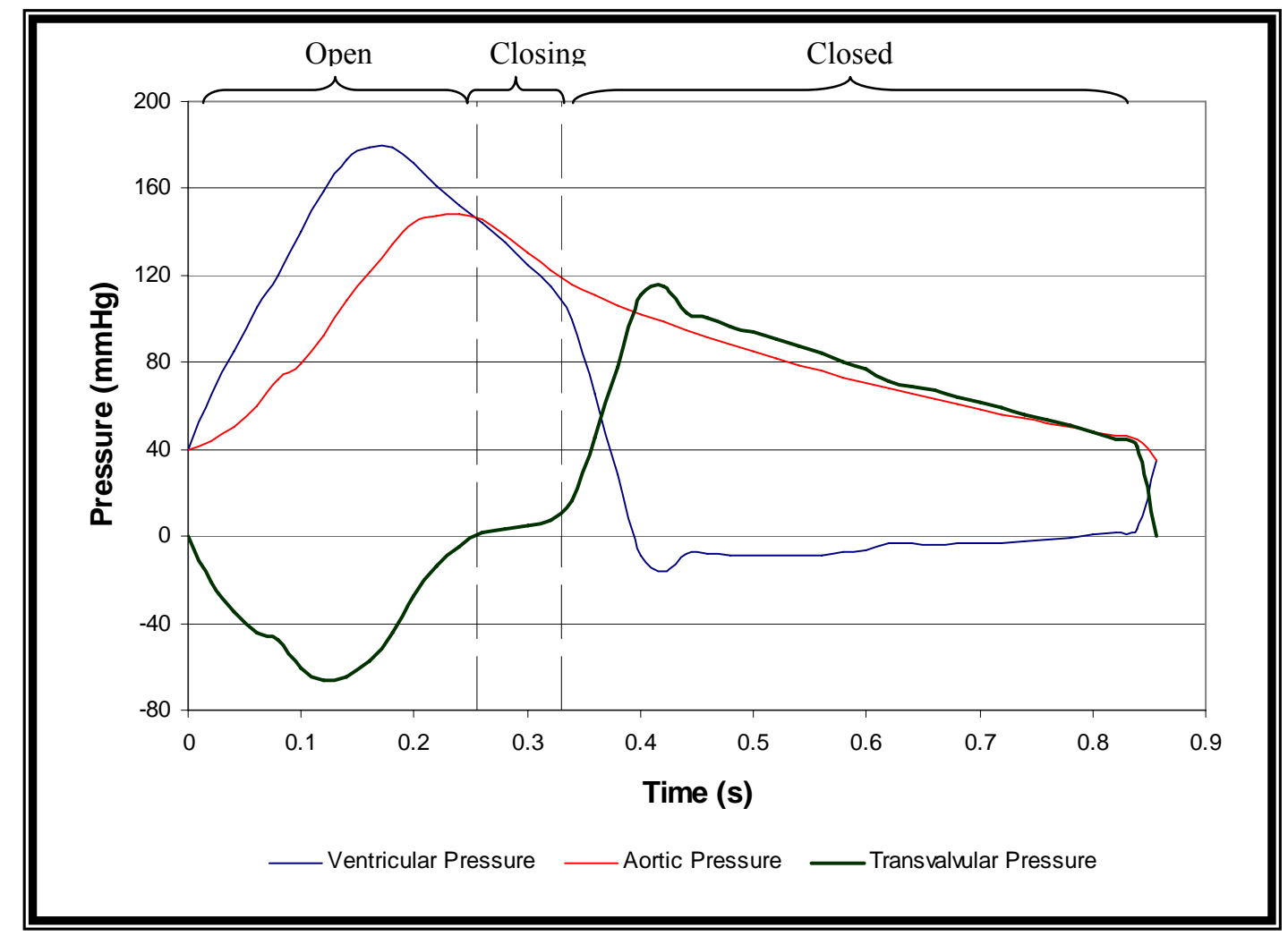

Figure 26. Characteristic aortic, ventricular, and transvalve pressure waveforms for a BARD reinforced SIBS trileaflet valve

\subsubsection{Elements and Mesh Optimization}

Heart valve leaflets, in general, are thin walled structures, and for the $19 \mathrm{~mm}$ TAD SIBS heart valves under investigation, the leaflet thickness ranged from $0.2-0.4$ $\mathrm{mm}$. The three types of elements that have been used to model heart valve leaflets in the past include continuum (solid) elements, membrane elements, and shell elements [63]. If 
one were to model the leaflet with continuum elements, the element size would have to be very small due to the material thickness, resulting in the need for a high mesh density and, therefore, a computationally inefficient simulation. In addition, to model the through-thickness stress distribution, several elements would be required through the thickness, resulting in an even higher mesh density. As a result, characterizing the stress distribution through the leaflet with continuum elements is unlikely. Membrane elements allow the simulation of a thin-walled structure such as a leaflet, but these elements have no bending stiffness; therefore, they are not appropriate for use in a heart valve leaflet that is being modeled through any bending criteria, such as those that occur during systole. Shell elements also allow the modeling of thin structures; however, these elements can simulate bending events. In addition, the shell element has multiple integration points through its thickness, so the stress variation through the thickness can be quantified. As a result, shell elements were determined to be most appropriate for modeling the SIBS valve leaflets, resulting in the selection of an ABAQUS-type S4R element. The S4R element is a general purpose shell element that allows transverse shear deformation. It is a finite-membrane-strain, reduced integration, quadrilateral shell element that is suitable for modeling contact.

The valve stent could not be considered as a thin-walled structure, so it was modeled with the C3D8R ABAQUS element type. This is an eight node linear brick element with reduced integration and hourglass control. The S4R and C3D8R element types are compatible when applying a tie constraint between shell and continuum elements. 
Although ABAQUS is equipped with an adaptive remeshing capability where it can iteratively improve the mesh in order to achieve a more accurate solution, it was found to be more beneficial to perform a manual mesh optimization. Upon using adaptive remeshing, an unrealistically fine mesh was developed that would result in a computational time on the order of days. In order to perform the manual mesh optimization, four different models were run, and the mesh density was varied by changing the seeding density for the model. The four different seeding densities that were applied included $0.25,0.30,0.40$, and 0.50 accounting for a total mesh size of $18,594,13,407,6,882$, and 4,434 elements respectively. A quasi-static diastolic load was applied to the leaflet, and the models that were generated were compared by qualitatively evaluating the stress distribution trends over the leaflet and quantitatively comparing the magnitude and location of the maximum stress. On qualitative analysis, all four models had the same stress distribution trends over the leaflets and stent. By comparing the percent difference between the maximum stresses, a $1.35 \%$ difference existed when the seeding density was changed from 0.25 to 0.3 , a $7.3 \%$ difference existed when the seeding density was changed from 0.3 to 0.4 , and a $6.24 \%$ difference existed when the seeding density was changed from 0.4 to 0.5 . The computational time ranged from 30 minutes to $24 \mathrm{hrs}$ for the minimum and maximum mesh densities, respectively. As a result, a 0.35 seeding density was chosen to maximize computational efficiency while maintaining accuracy. This was not one of the seeding densities analyzed, but the $0.3-$ 0.4 seeding density range was determined to be sufficient as it resulted in less than a $10 \%$ change to the maximum stress. 


\subsubsection{Solution Method}

A quasi-static analysis technique was implemented to model each valve type presented in Table 7. The quasi-static analysis was carried out utilizing the implicit method, and the valve was subjected to diastolic loading from $0 \mathrm{MPa}$ up to the maximum diastolic load, along the characteristic loading curve (Figure 26).

\subsubsection{Model Validation}

\subsubsection{1. $\quad$ Transient Geometry Analysis}

The transient geometry of the PET Knit 2 (BARD) - reinforced valve prototype (listed in Table 5 and shown in Figure 27) was investigated using the Vivitro Left Heart Simulator, dual camera stereo photogrammetry (DCSP), and surface reconstruction. The information obtained from the transient geometry analysis method was used to validate the corresponding finite element model. The valve was mounted in the aortic position of the Vivitro Left Heart Simulator (refer to section 4.2.3), and leaflet motion was measured under normal physiological flow conditions, with a heart rate of 70 beats/min, a cardiac output of 5.6 liters/min, and a pulse pressure of $120 / 80 \mathrm{mmHg}$. A time series of photographs was taken during both the opening and closing phases, and image reconstruction allowed the quantification of leaflet displacement during the cardiac cycle.

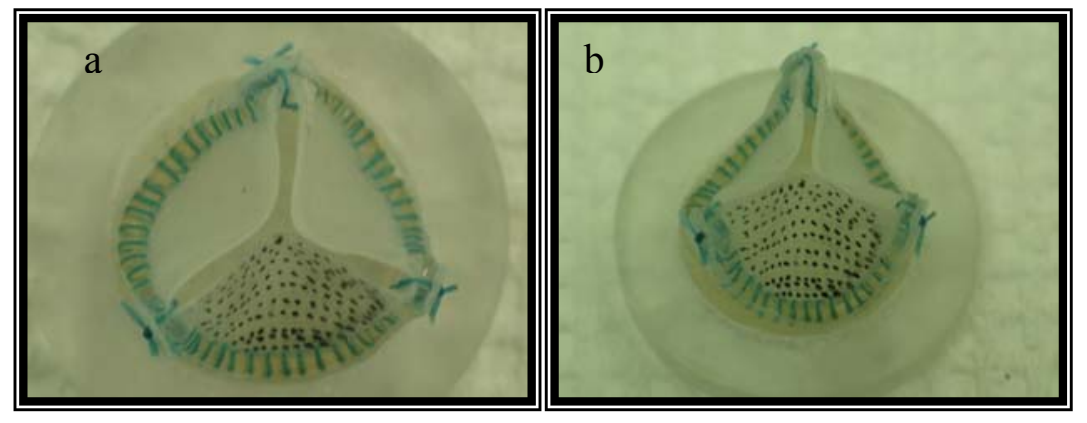

Figure 27. BARD-reinforced SIBS valve with medium coaptation curvature and $48.5 \%$ styrene stent. Image shows (a) top and (b) oblique view including the leaflet marked with approximately 120 fiducial points. 


\subsection{Image Acquisition}

Dynamic leaflet motion was monitored using the DCSP method described by Gao et al. [60] and Lee et al. [45, 61]. This is a two-phase procedure, where two identical cameras are focused on the same object to simultaneously acquire images from two different angles. The 3-D coordinates of markers on the object were then calculated from each pair of simultaneous digital images.

The aortic valve mounting chamber of the Vivitro was modified to obtain an optical window into the aortic channel. An acrylic endoscope-mounting chamber was manufactured and attached to the exterior surface of the ventricular chamber to allow the endoscope tips to be bathed in the same blood-analog fluid that the valve was exposed to, thereby assuring the same refractive index in both the valve and endoscope environments for image acquisition (Figure 28a-c). The leaflet was marked with approximately 120 fiducial points of $0.2 \mathrm{~mm}$ diameter (Figure 27). Two Sony ST30 CCD cameras (Sony, San Jose, CA, USA) with attached Slim 12 Hawkeye Borescopes (Hawkeye Gradient Lens Corporation, Rochester, NY, USA) acquired images of a single valve leaflet at an angle of $30^{\circ}$ from the horizon and $30^{\circ}$ between each other (Figure 28). This orientation was chosen as it resulted in the best reconstruction of 3-D structures of known dimensions by Lee [61] and further verification by this researcher during reconstruction of gage blocks of known dimensions.

The Vivitro Left Heart Simulator was set to run at the physiological heart rate and cardiac output combination stated above, and a function generator was used to trigger both cameras to allow image acquisition by the frame grabber (Coreco Viper-Quad) at a frame rate of $2 \mathrm{~Hz}$. Simultaneously, the pressure and flow data for the valve was 
acquired with the MP100 data acquisition system and software (Biopac Systems, Inc., Santa Barbara, CA), and the trigger pulse was fed into this system via a pulse elongation circuit. By collecting the trigger pulse in combination with the pressure and flow data, the exact location of image acquisition during the cardiac cycle can be known. A pulse elongation circuit was required in order for the MP100 system to recognize the trigger signal. A fiber-optic light was employed to illuminate the field. A schematic of the image acquisition set-up is provided in Figure 29, and an example of the Biopac data, including the ventricular and aortic pressures, aortic flow, and trigger pulse, is provided in Figure 15.

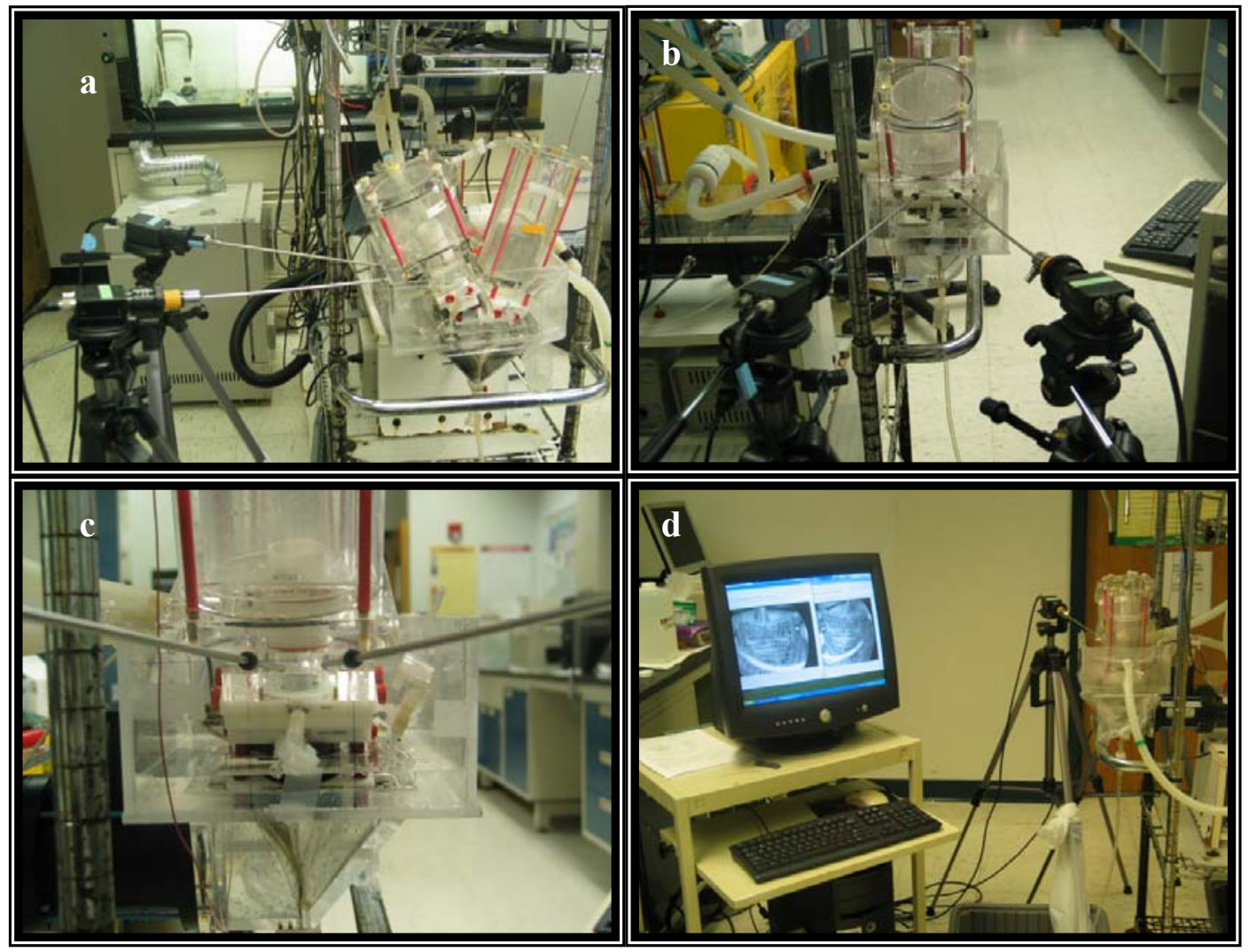

Figure 28. Image acquisition set-up showing (a) how the borescopes are inserted into the acrylic endoscope mounting chamber, (b) the orientation of each camera and associated borescope, (c) the location of the valve with respect to each borescope, and (c) the image generation on the imaging PC. 


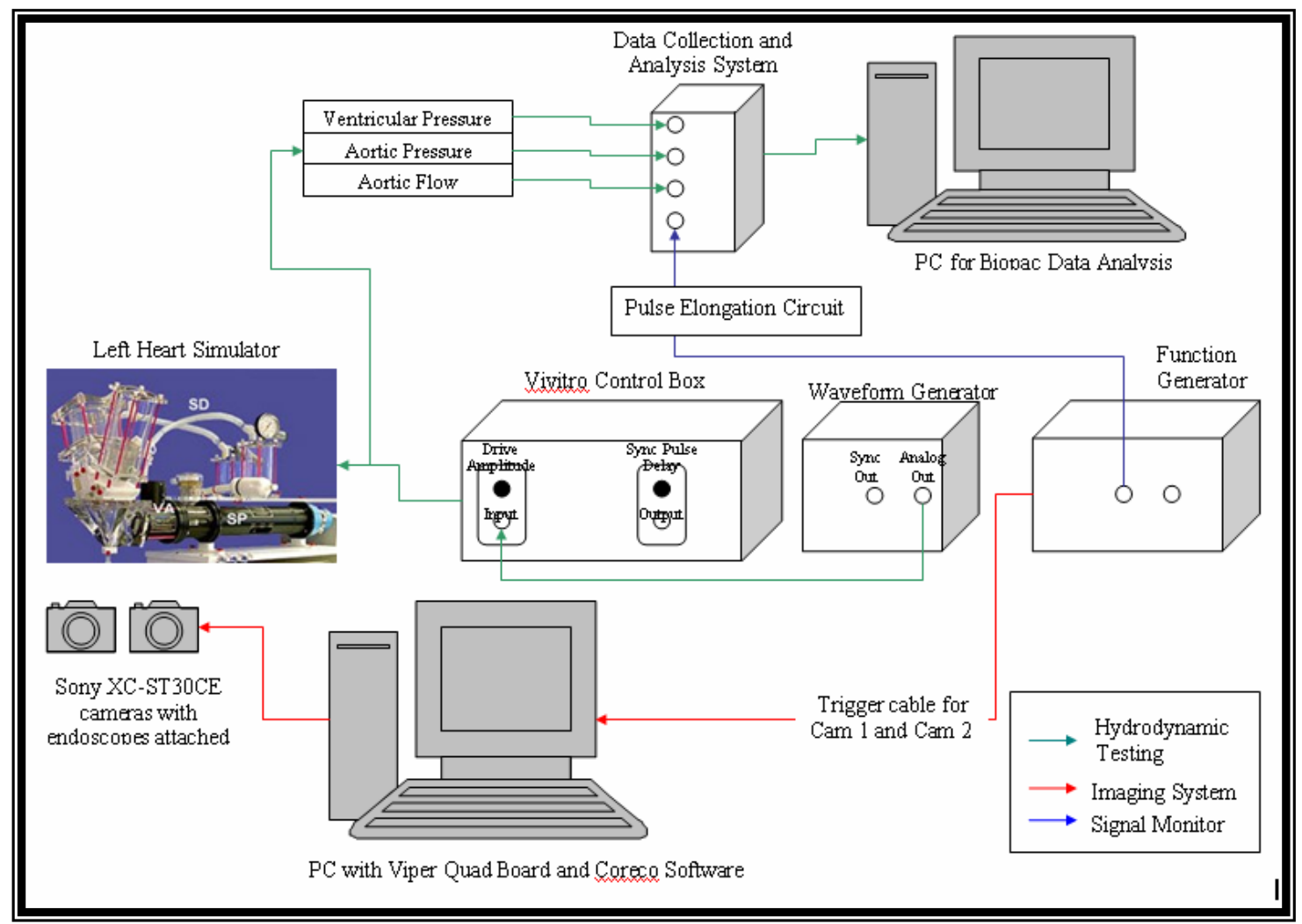

Figure 29. Schematic of the image acquisition system for imaging valves undergoing hydrodynamic testing.

\subsection{Image Reconstruction and Analysis}

From the acquired image sequences, image pairs were selected that represented a total of 8 different locations during the cardiac cycle. In addition, a baseline image pair was acquired, which represented the leaflet geometry under no load. The 3-D coordinates of the marker matrix were derived using Photomodeler ${ }^{\circledR}$ (Eos Systems Inc., Vancouver, BC), and these were used to reconstruct the contour of a leaflet during the opening and closing phases of the cardiac cycle. A MatLab code was employed to reconstruct the scatterplot of the marker matrices for qualitative comparison of the general leaflet geometry. For quantitative validation of the model, three select points were chosen, and the distances between each pair was computed from the knowledge of 
their $\mathrm{x}, \mathrm{y}$, and $\mathrm{z}$ coordinates (Figure 30). The same approximate three nodes were selected from the finite element model, and the distances between each were computed. This was repeated at each time step corresponding to the 9 image pairs obtained experimentally, and the experimental and model values were compared. The three particular points were selected to represent three different areas within the valve that produce different phenomena during cycling. Distances between points were chosen to validate the model, rather than displacement of the points with respect to the unloaded case, as Photomodeler ${ }^{\circledR}$ lacked the capability of providing a uniform coordinate system from one image pair to the next. As a result, there were slight variations between the coordinate systems of each consecutive image pair, and the coordinates of points obtained from one image pair could not be associated with those from a different time step. Another short-coming of the procedure was that it was not always possibly to have the exact same time points represented for both the model and experimental data. The closest time point available from the model results was used for comparison with the experimental.

\subsubsection{2. $\quad$ Stent Deflection}

To further validate the model, stent deformation during the cardiac cycle was measured by imaging the BARD valve from above. Images were processed in Adobe ${ }^{\circledR}$ Photoshop ${ }^{\circledR}$, where the distance between each adjacent stent post was quantified (Figure 31). Each image was overlaid with a grid, and the grid was scaled with the known dimensions of the valve in the horizontal and vertical directions. Dimension lines were drawn between each adjacent stent post, and the horizontal and vertical offset from one post to the next was measured. The distance between posts was then calculated using 
Pythagorus' theorem. This dimension was then compared with that from the output of ABAQUS at the corresponding point in the cardiac cycle.
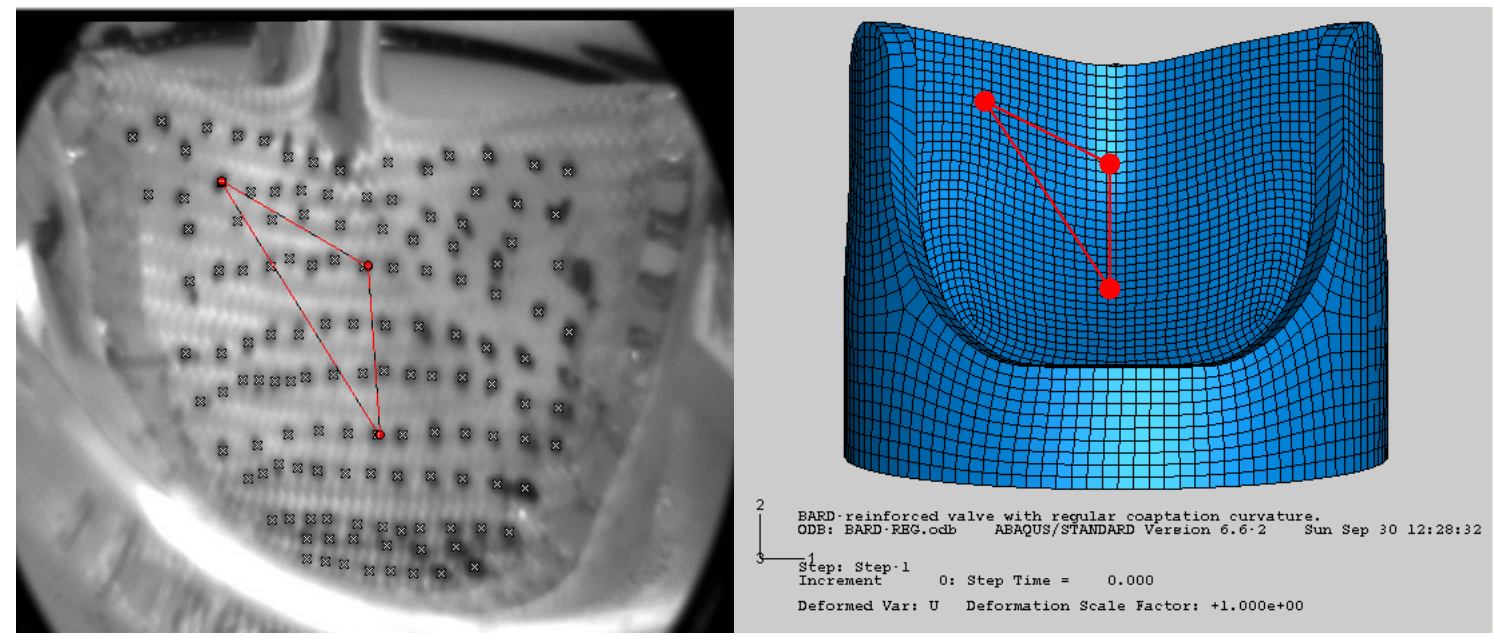

Figure 30. Quantitative validation of point-to-point distances between the experimental DCSP method and the finite element model. Points were selected on the image, and the distances between each was computed from the knowledge of their 3-D coordinates. The corresponding points were selected on the corresponding finite element model (right), and the distances between each were computed for comparison.

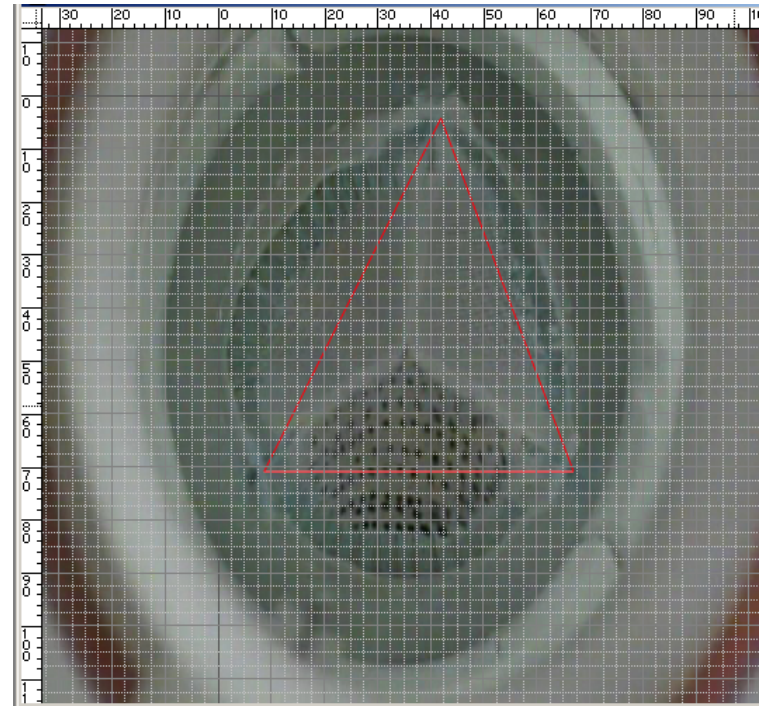

Figure 31. Photoshop ${ }^{\circledR}$ image showing grid overlay and stent post dimension lines. 


\subsection{Specific Aim 3 - Durability Assessment}

The prediction and/or quantification of durability are vitally important for the development of devices exposed to cyclic fatigue as premature failure will have catastrophic results. For heart valves, failure will certainly result in death to the patient if warning signs are not evident and failure is instantaneous. Characterization of the durability of a valve is not simply a requirement established by the FDA, it is a necessity when developing a device for anticipated long-term fatigue. Cyclic testing under simulated physiological conditions can provide a wealth of knowledge as to the failure modes and lifespan of a device, and can guide future design and development. In vitro accelerated fatigue can provide evidence as to the anticipated durability of a device, but the only conclusive method to evaluate its true durability is to place it in the intended use environment, where mechanical and biological mechanisms work in collaboration. Nevertheless, comparative in vitro testing during device development can certainly guide design by indicating potential weaknesses in the design. The downfall to in vitro testing is that it is time-consuming, so a modeling approach to fatigue prediction is proposed for incorporation into the design and development process of the SIBS trileaflet valve.

\subsubsection{Accelerated Fatigue Testing}

\subsubsection{1. $\quad$ Cyclic Tensile Testing}

Tension fatigue tests were performed according to ASTM standard D 3479M 96, using the ELF materials tester. The materials' properties were evaluated in air and under load control, where loading amplitude was set at $\pm 10 \%$ of the mean load. The load magnitude used for each specimen was progressively lowered from the static failure magnitude until a sufficient amount of data points existed to create an S-N curve. A load 
frequency of $100 \mathrm{~Hz}$ was adopted. Cycling was continued until failure, which was defined as a strain of 0.5 . If a specimen reached 350 million cycles (equivalent to 8.75 yrs) without failure, the test was stopped and the sample was considered to have an infinite life for that stress as it appeared to be below the endurance limit. Although mechanical valves need to prove to be durable for an equivalent of 600 million cycles (15 yrs), it will take 70 days to reach this point at a cycling rate of $100 \mathrm{~Hz}$, so it was assumed that an equivalent of $8.75 \mathrm{yrs}$ of fatigue was sufficient for this case.

As with the static tensile tests, leaflets were fabricated in the same manner as for valve fabrication, and radial, circumferential, and samples $45^{\circ}$ between those two were stamped out and tested. The resultant S-N curves were fit to logarithmic trend lines for input into the fatigue model.

\subsubsection{2. $\quad$ Valve Durability Testing}

Accelerated fatigue testing of valves was carried out on a Vivitro Systems HiCycle accelerated fatigue tester (VSI HCS4991, Vancouver, British Columbia) (Figure 32). Prior to placement in the Hi-Cycle, valves were catalogued in terms of their lot number, design specifications, sterilization history, overall quality, and hydrodynamics. Valves underwent hydrodynamic evaluation, as per procedure 4.2.3. This served as baseline data for comparison with incremental fatigue time-points.

Valves were secured in the Vivitro Hi-Cycle in the orientation shown in Figure 32. Six valves can be tested simultaneously, but in order for the system to remain balanced and all of the valves to be subjected to the same testing conditions, the valves must be equivalent in both size and hydrodynamic properties. Valves were cycled at $1200 \mathrm{cycles} / \mathrm{min}(20 \mathrm{~Hz})$ at a backpressure of $90+20 /-0 \mathrm{mmHg}$, which is specific for the 
testing of aortic valves. The amplitude of the system was adjusted to provide complete valve opening and closing, which was verified with that during hydrodynamic testing at $70 \mathrm{BPM}$ and $5.6 \mathrm{~L} / \mathrm{min}$. Incremental checkpoints for the valve included a 1-2 day evaluation of transvalvular pressure drop, a macroscopic evaluation every 25 million cycles, and a microscopic and hydrodynamic evaluation every 75 million cycles. Valve failure was defined when during hydrodynamic testing, the transvalvular pressure drop and regurgitation increased to more than three times that of the pre-fatigue evaluation or if upon qualitative examination, the valve receives a rating of 5 as specified in Appendix III: Table 18. Valves that failed were catalogued photographically, and their failure mechanism was reported.

The Hi-Cycle system comes equipped with a temperature controller, which allows the working fluid $(0.2 \%$ glutaraldehyde/water solution: glutaraldehyde is an effective bacteriostat) to be maintained at $37^{\circ} \mathrm{C}$ throughout the testing procedure. Inline pressure transducers (Utah Medical 6069) allow for rapid transvalve pressure monitoring through the use of the monitoring software included with the test system (Vivitest Software, Vivitro Systems). The FDA and ISO 5840 specifications state that the transvalve pressure drop must be maintained over $95 \%$ of the cycles. A typical plot of a tuned chamber is provided in Figure 33, showing a peak pressure drop of $90 \mathrm{mmHg}$. The peak mmHg, measurement on the lower left hand side shows the average pressure drop measured during the recording period. When this value is in red, the specified pressure drop is not achieved over $95 \%$ of cycles. When this value is in green, however, it is. 


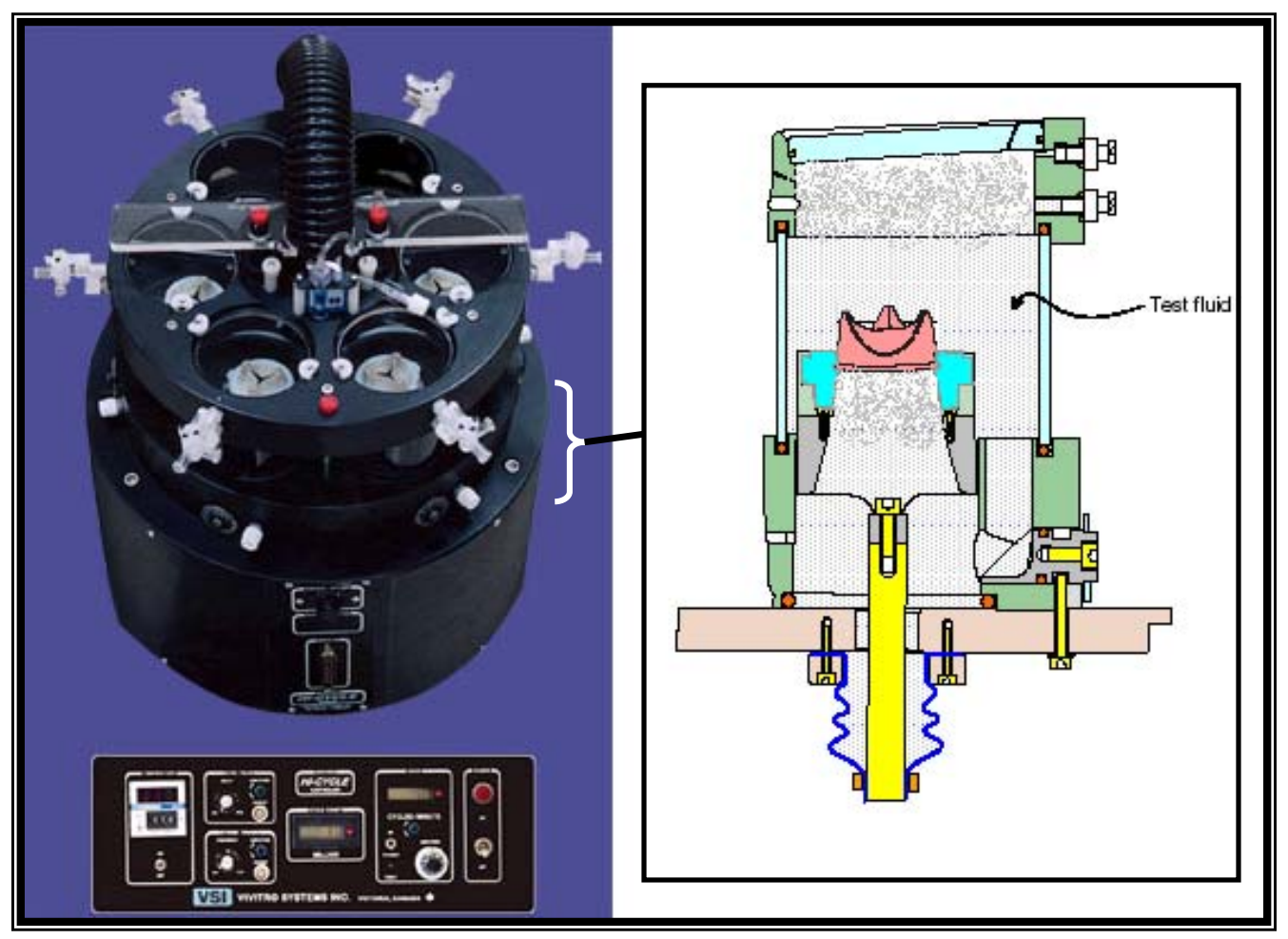

Figure 32. Vivitro Systems Hi-Cycle including schematic of chamber where valve is seated.

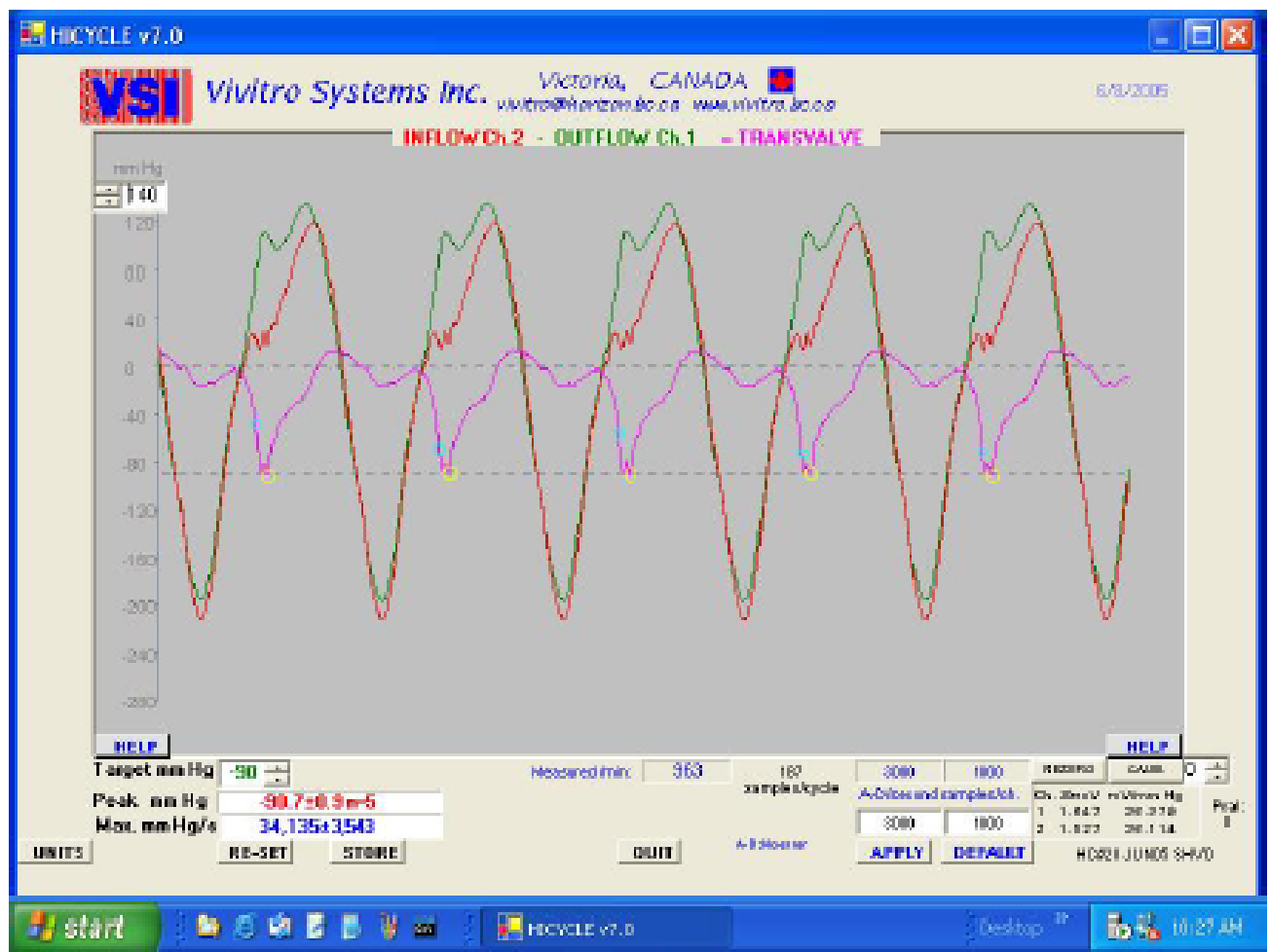

Figure 33. Vivitest software for the acquisition, analysis, and display of heart valve test data showing the inflow, outflow, and transvalve pressure, where the peak transvalve pressure is at $90 \mathrm{mmHg}$. 


\subsubsection{Fatigue Prediction}

An initial fatigue predictive model is proposed for the cyclic tensile fatigue of flat leaflet samples evaluated in section 4.4.1.1. The initial production of an S-N curve is required to assess the relationship between mean stress and cycles to failure. It is this relationship that can be incorporated into a predictive model to assess for the impact of varying stress conditions on the fatigue life of the sample.

The heart valve leaflets undergo complex loading as the valve alternates between tension and flexion during one cardiac cycle. The period in which these alternate loading conditions are applied is highly dependent on the heart rate: as the heart rate increases, the diastolic loading period decreases. Initially, the model proposed above will be adapted for the trileaflet valve, where only loading during diastole will be accounted for. The stress history obtained in the finite element model (section 4.3.2) will be incorporated into the fatigue lifetime prediction model for solution, and the impact of tension fatigue will be examined. The derived models will be validated by experimental results from the accelerated fatigue testing of valves in the Vivitro Hi-Cycle (section 4.4.1.2). 


\section{RESULTS AND DISCUSSION}

This section is divided into three primary parts: the first involves the preliminary evaluation of valve design to assess the appropriate leaflet-stent attachment technique and leaflet reinforcement material properties; the second covers a rigorous assessment of potential reinforcement materials and design parameters for leaflet stress minimization and improved durability; and the third involves the implementation of a fatigue prediction model. By separating this section into the above-mentioned parts, the motive behind extreme design changes can be made apparent, and the limitations of potential reinforcement materials can be understood.

It should be noted that all valve and valve components were manufactured by the same technician. In so doing, the manufacturing process could remain consistent, and differences could be attributed to design variations as opposed to technician-related changes.

\subsection{Preliminary Evaluation for Valve Design}

There were three preliminary valve design iterations that were evaluated for in vitro hydrodynamics and accelerated fatigue (Design 1 - Design 3 Table 3). The most successful of each of these designs were utilized in simultaneous in vivo studies. Each of the valves tested were the specified size for testing in an ovine model, that is, they all had a $19 \mathrm{~mm}$ tissue annulus diameter (TAD). For design 2, a variety of leaflet reinforcement materials and manufacturing techniques were instituted to assess their impact on valve durability, thereby refining what characteristics for leaflet design are optimal.

Accelerated fatigue testing of valves began with Design 1, which consisted of an open polyester (PET) mesh (LARS ${ }^{\circ}$ mesh) that was sandwiched between $\mathrm{SIBS}(8.5 \%$ 
styrene). The failure mechanisms associated with Design 1 (refer to section 5.1.2) resulted in the need for a more dense reinforcement fabric and a more secure leaflet stent attachment technique. As a result, a combination of different PET reinforcement fabrics and polymer coatings were selected for use in Design 2. The reinforcement fabrics included the original LARS ${ }^{\circledR}$ mesh (as a baseline comparison), a more dense Athletic mesh, a weave, and a Dacron knit. The polymer coatings included the original $8.5 \%$ styrene coating and a more porous $16 \%$ styrene coating. It was hypothesized that the porous coating would provide less resistance to flexion, thereby improving valve hydrodynamics. Design 2 leaflets were made by heat-sealing the flat fabric samples into a cylinder with the maximum stretch oriented circumferentially. The fabric was dipcoated twice in the particular polymer solution, and the leaflet cylinder was attached to the high-profile, flexible, $30 \%$ styrene stent by means of sutures. The trileaflet geometry was attained by thermal forming with three spherically shaped mandrels.

The Dacron reinforcement was found to provide the longest fatigue life (refer to section 5.1.3) in combination with the $8.5 \%$ styrene coating. As a result, Design 3 was manufactured in much the same manner as Design 2, except that the leaflet surface was smoothed by the addition of a compression molding process, and the stent stiffness was increased through a combination of geometry changes and the use of a $48.5 \%$ styrene SIBS.

Each of the valves underwent accelerated fatigue testing in the Vivitro Hi-Cycle, and the three most successful designs were selected for in vivo implantation [14]. Although the in vivo studies are not a part of this particular investigation, it must be noted 
that important findings during these studies have contributed to design changes, and they will be noted in the text.

\subsubsection{Hydrodynamic testing}

Hydrodynamic testing of the Design 1 - Design 3 valve iterations was carried out according to the methods presented in Section 4.2.3. It is quite obvious from the results presented in Figure 34 and Figure 35 that a progressive improvement was made in the hydrodynamic function from one design to the next; there was a decrease in the transvalvular pressure drop and percent regurgitation with each new design.

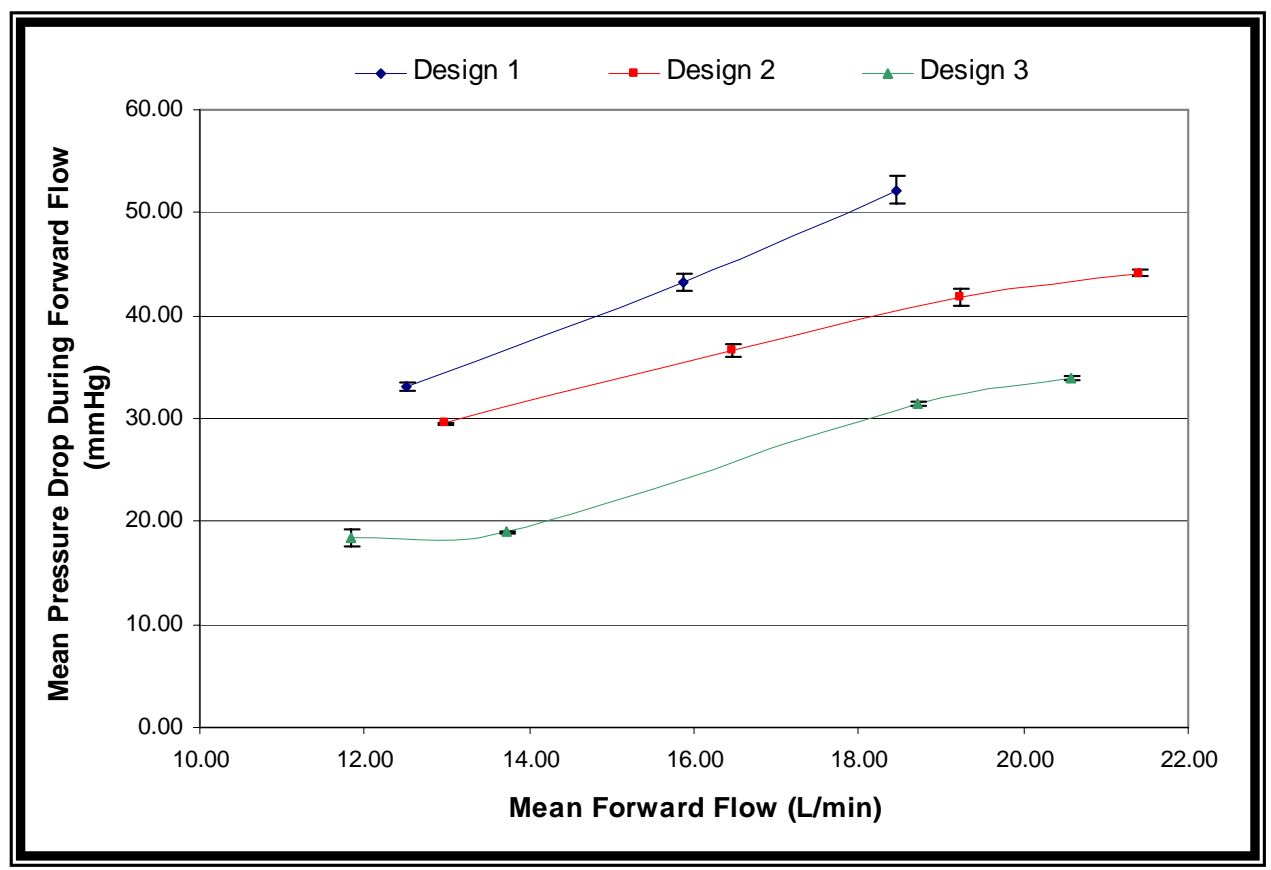

Figure 34. Mean transvalvular pressure drop during forward flow for SIBS valve designs 1, 2, and 3.

A decrease in the pressure drop from Design 1 to Design 2 can be explained by the initial valve geometry. The unstressed valve geometry for Design 1 is spherical, so in order to open the valve, a complete inversion of curvature is required. For the two 
designs of cylinder valves, the curvature of the leaflet is not as extreme; therefore, less force would be required to invert this curvature during systole.

As far as the regurgitation, design changes to the coaptation region can impact regurgitation, especially if these changes prevent efficient coaptation. In the Design 1 valve, the coaptation region is completely flat, and for Designs 2 and 3, a slight curvature is incorporated. Design 2, however, has a more flexible stent; therefore, more internal flexion of the stent occurs during diastole, making coaptation different from that of Design 3.

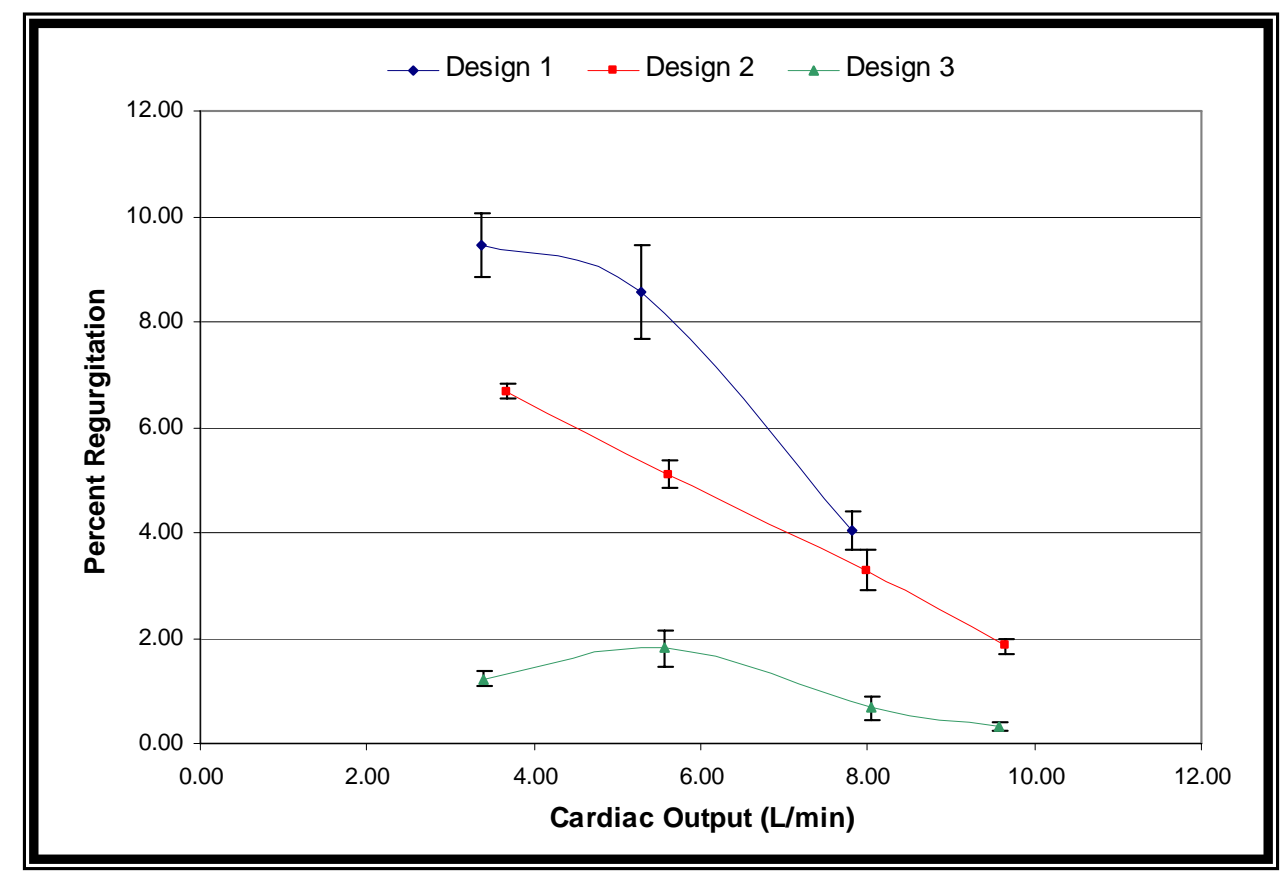

Figure 35. Percent regurgitation measured while the valves are closed and closing for SIBS valve designs 1,2 , and 3 .

\subsubsection{Design 1}

Design 1 valves that were exposed to accelerated fatigue failed at an average of 0.628 years as a result of leaflet stent detachment and polymer degradation/blowout from 
between the mesh interstices (Figure 36). The leaflets were bonded to the stent with the same polymer solution used for leaflet dip-coating, which proved to be insufficient. Even if the bond was reinforced, the problem remained that the polymer between the mesh interstices that was unreinforced did not have the structural integrity to withstand the load acting on it, resulting in the polymer blowout that was seen.

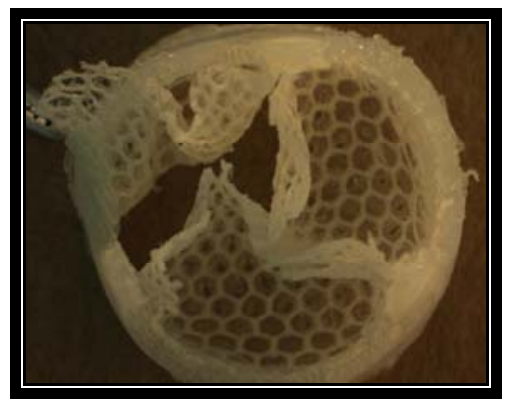

Figure 36. Design 1 valve after an equivalent of 0.628 years of cycling. Failure was as a result of leafletstent detachment and polymer blowout from between the mesh interstices

\subsubsection{Design 2}

Based on the failure mechanisms of Design 1, two major design changes were made to the polymer valve. Firstly, the use of polymer bonding between the leaflet and stent was replaced with sutures to allow a more secure attachment. Secondly, varying reinforcement fabrics were investigated for use in this design. The first prototype valve incorporated the original LARS ${ }^{\circledR}$ reinforcement. Failure occurred at an equivalent of 0.908 years of cycling as a result of polymer blowout from between the mesh interstices (Figure 37a). This design adjustment did result in an increase in valve durability, but the reinforcement mesh was insufficient. As a result, a more dense reinforcement fabric was required, and this prompted the simultaneous testing of a dense mesh (Athletic mesh), a 
weave, and a knit (Dacron). The processing technique for each was the same, but accelerated fatigue of these samples resulted in failure due to varying mechanisms. The weave-reinforced valve failed at an equivalent of 0.943 years as a result of fraying at the free edge and fabric separation at the leaflet base (Figure 37b); the Athletic meshreinforced valve failed at an equivalent of 1.38 years due to polymer degradation from between the larger interstices and actual mesh fracture at the leaflet belly (Figure 37c); and the Dacron reinforced valve failed at an equivalent of 10.35 years as a result of a 3fold increase in transvalvular pressure drop when compared with pre-fatigue hydrodynamic testing (Figure 38). It must be noted that the weave was oriented so that the fibers ran in the circumferential and radial directions, so along the free edge, individual fibers had nothing but the polymer matrix to hold them in place. From Figure 38, distinct deposits are seen on the Dacron leaflet surface, so the valve was dissected and sent for SEM (Figure 39). Non-fatigued samples (Figure 39a), fatigued samples (Figure $39 \mathrm{~b}$ ), and samples that had been fatigued and then processed in toluene were evaluated (Figure 39c). The toluene effectively dissolved the polymer coating, but even when the polymer was removed, the deposits still remained. An elemental analysis performed by SEM-EDS revealed that the deposits were in fact silicone, which is not a constituent of the valve itself; therefore, it was concluded that valve failure was as a result of silicone deposits that had leached from the testing system and was not design related.

The natural heart valve leaflet is more compliant in the radial direction than in the circumferential direction (Figure 40a), but the particular orientation that was used for each of the valves thus far showed opposite trends (Figure 40b). Further Dacron valves were manufactured where the fabric orientation was switched to allow greater 
compliance in the radial direction. These valves failed at an equivalent of 2.72 (Figure 41a) and 6.12 years (Figure 41b) as a result of stent fracture and hole formation in the mid-leaflet region, respectively. It was concluded that the increased stiffness in the circumferential direction resulted in higher stress concentrations in both the stent and leaflet, resulting in the modes of failure seen.

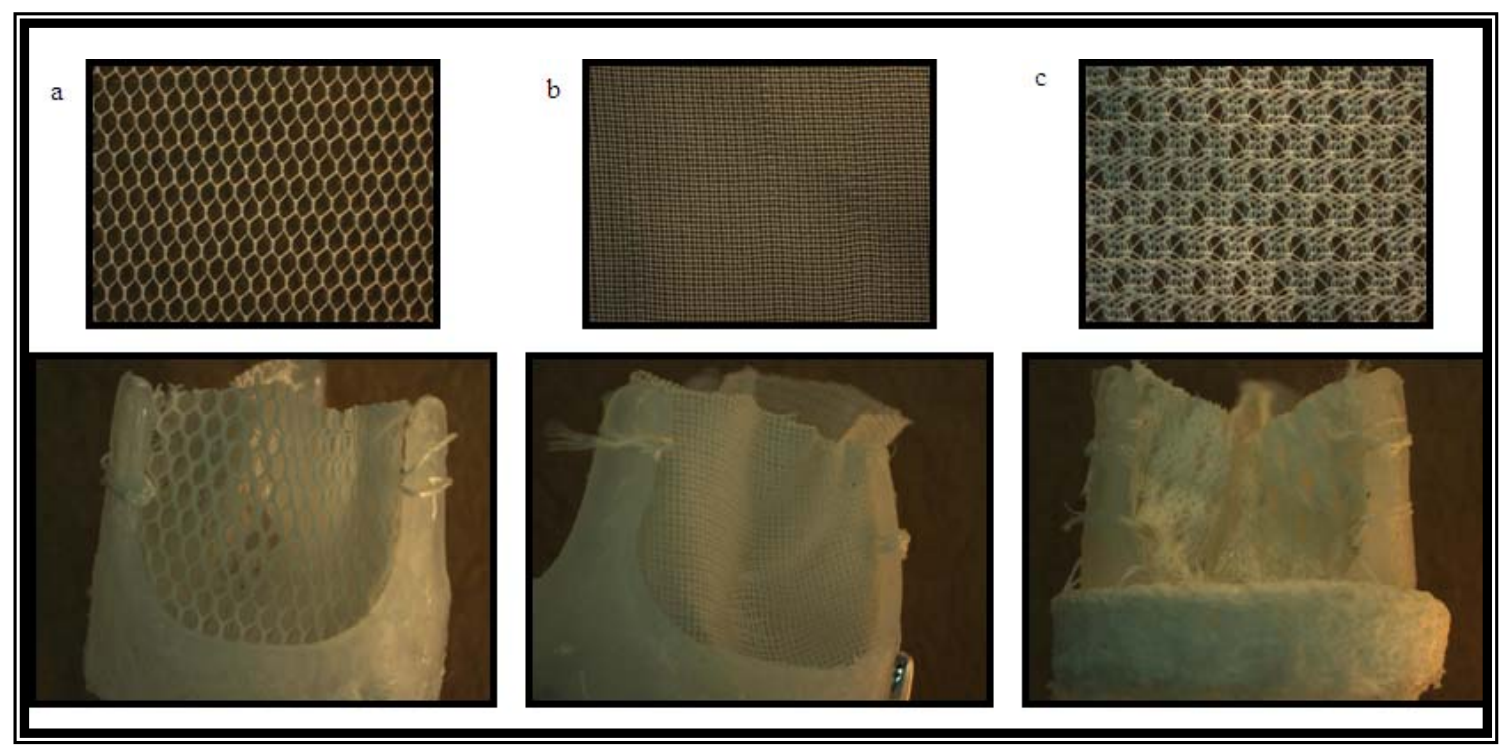

Figure 37. Varying iterations of the dip-coated thermal-formed design 2 valves including different polyester reinforcement fabric: (a) LARS® reinforcement mesh failure mode was polymer blowout from between mesh interstices at an equivalent of $0.908 \mathrm{yrs}$, (b) PET weave failure mode was weave degradation at an equivalent of 0.943 years, and (c) athletic mesh failure mode was polymer and mesh degradation at an equivalent of 1.38 years.

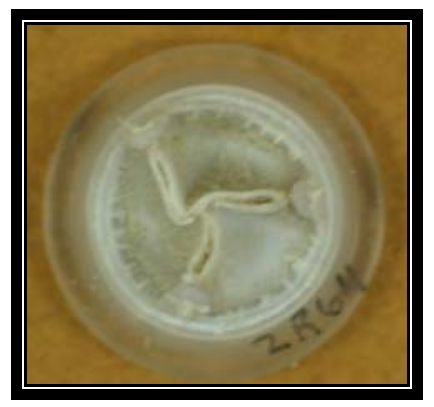

Figure 38. Dacron reinforced valve with maximum stretch oriented circumferentially. Note the surface deposits on the leaflet that resulted in a 3-fold increase in transvalvular pressure drop at 10.35 years. 


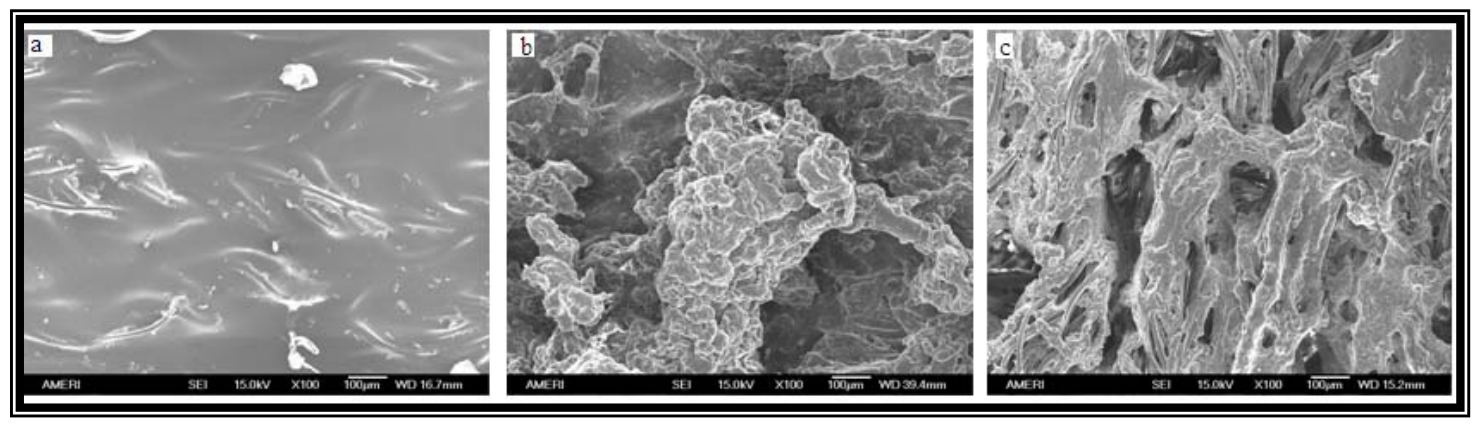

Figure 39. SEM images of the Dacron-reinforced valve shown (a) prior to cycling, (b) after cycling an equivalent 10.35 years, and (c) after cycling an equivalent 10.35 years and subsequent polymer dissolution. The silicone deposits are denoted by the white arrows.

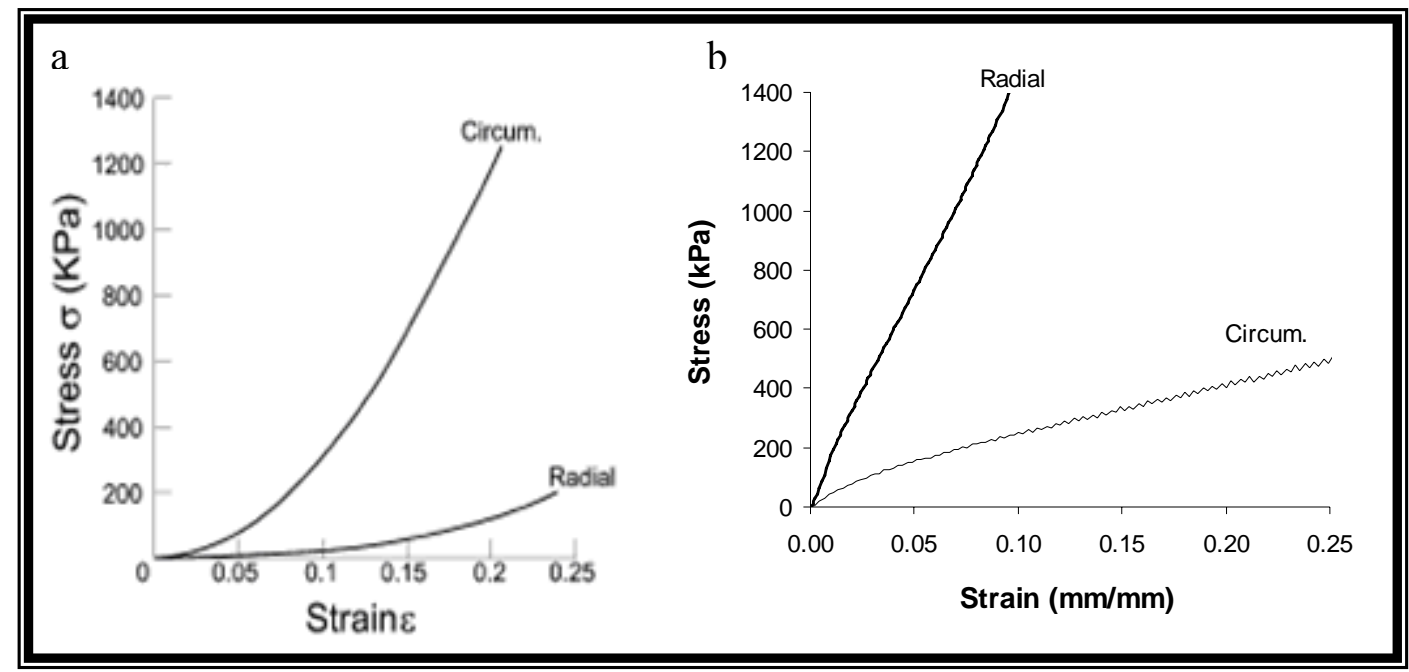

Figure 40. Stress-strain relationship in the radial and circumferential direction for (a) natural porcine valve leaflets (adapted from Li et al. [50]) and (b) dip-coated JSI leaflets with maximum elongation oriented circumferentially.

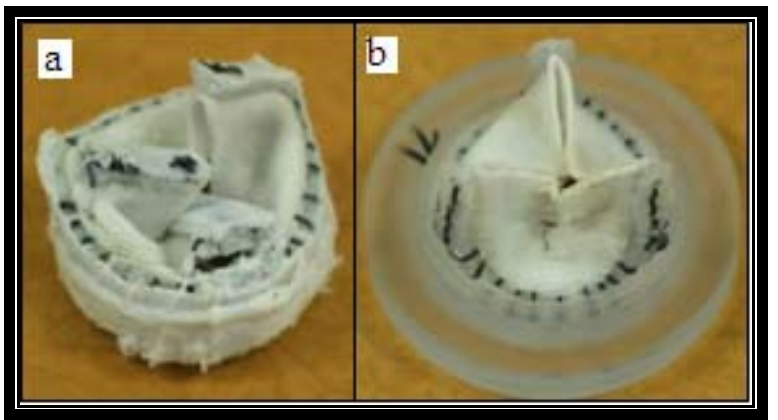

Figure 41. Dacron-reinforced valve with maximum stretch oriented in the radial direction. The failure mode was a combination of stent fracture (equivalent 2.72 years) and hole formation (equivalent 6.12 years) in the belly of the leaflet as a result of unphysiologically high stress in the circumferential direction. 
A further assumption was made that by switching from the non-porous coating with an $8.5 \%$ styrene SIBS to a porous coating with a $16 \%$ styrene SIBS, an improvement in the hydrodynamic function of the valve could be achieved due to greater leaflet flexibility. While the change in polymer had minimal impact on the hydrodynamic function of the valve, the increase in SIBS stiffness due to the increased styrene content resulted in the premature formation of cracks as shown in Figure 42. The increased styrene content appeared to result in crack formation orthogonal to leaflet bending.

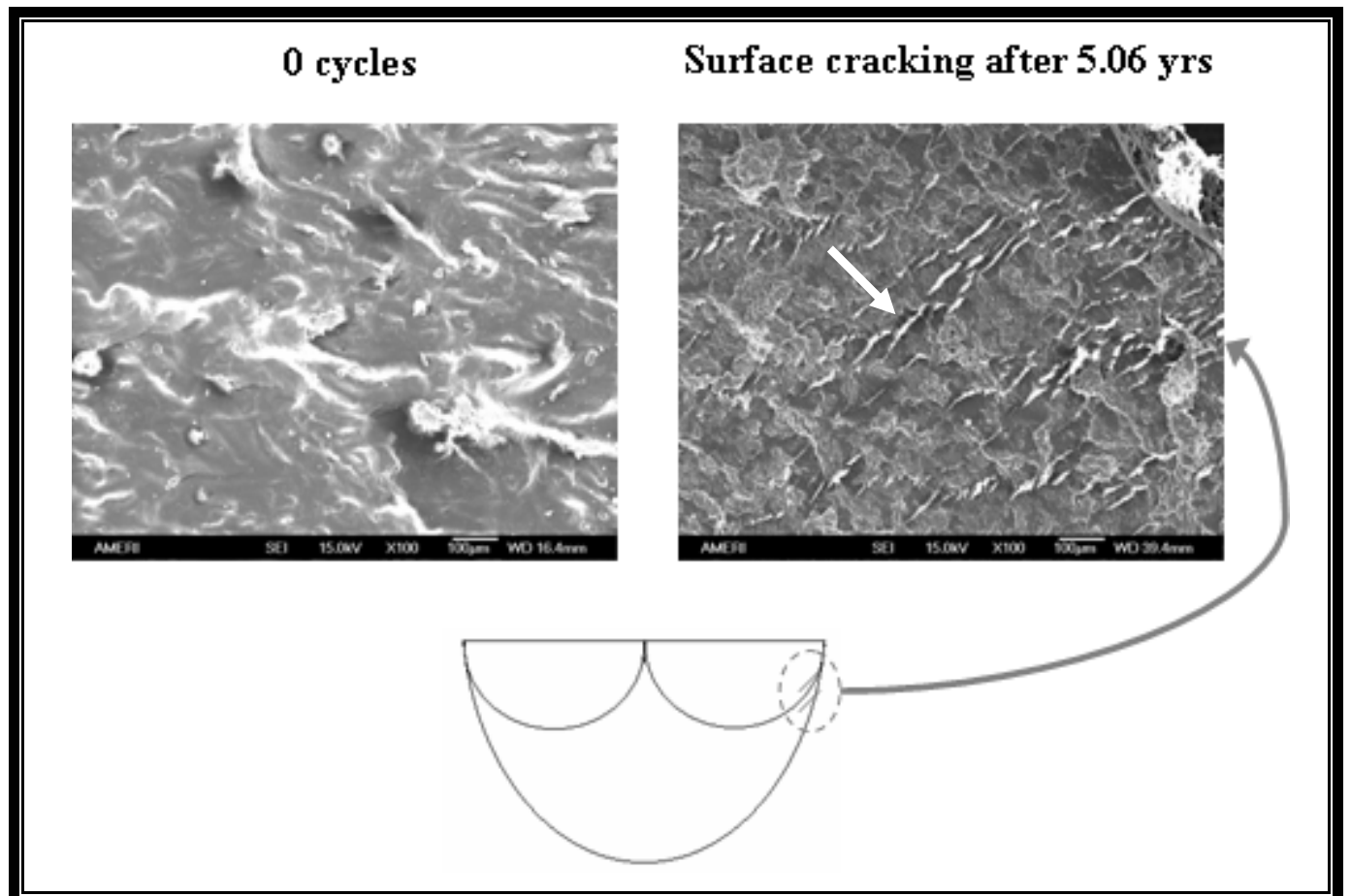

Figure 42. SEM image of aortic leaflet surface of a Dacron cylinder valve dip-coated in a porous $16 \%$ styrene SIBS solution. On the right image, cracking is seen after cycling an equivalent of 5.06 years. The sketch identifies the location of the crack formation. A white arrow points to some instances of crack formation. 
Based on these results, the dip-coated SIBS (8.5\% styrene) with circumferential stretch appeared to have the most promising durability, and it was this valve that was chosen for in vivo evaluation. Valve failure in vivo was as a result of excessive stent flexure and severe tissue overgrowth. The tissue overgrowth was originally hypothesized to be as a result of the surface roughness: the dip-coating procedure results in a polymer surface that follows the topology of the underlying Dacron, and it is for this reason that Design 3 incorporated a compression-molding technique to smooth the leaflet surface. In addition, the stent stiffness was increased and the profile reduced to minimize stent flexibility.

\subsubsection{Design 3}

The manufacturing procedure adopted for Design 3 initially included the pure compression molding of a 3-D laminate. Accelerated fatigue of this design showed failure at 0.635 years as a result of tear formation at the center of coaptation (Figure 43). An SEM image of the leaflet cross section (Figure 44) showed that the Dacron mesh was highly compressed between the two polymer layers, potentially resulting in increased residual stress in the leaflet fabric that could have contributed to premature failure. The compression molding process resulted in a decrease in the circumferential compliance of the leaflets as determined through qualitative stretching.

Further developments to smooth the surface of the leaflet involved a fusing of the dip-coating and compression-molding techniques, referred to as a hybrid. This hybrid technique incorporated the initial dip-coating of the leaflet and then compression-molding at a reduced pressure when compared with the purely compression-molded valve. This hybrid valve had favorable hydrodynamics as reported above, but failure of the valve 
occurred at an equivalent of 1.5 years due to tear initiation at the heat-sealed joint (Figure 45). The heat sealed joint at this stage had been reduced in cross-sectional area when compared with Design 2 for aesthetic reasons, but the failure mode resulted in the return to the original heat-seal geometry.

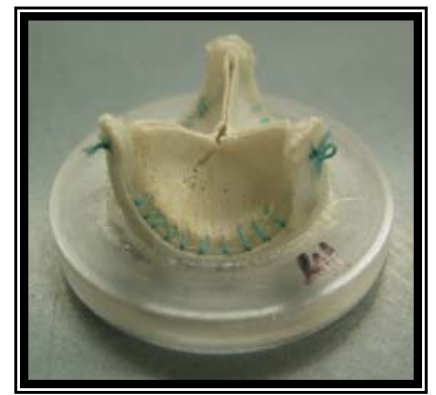

Figure 43. Dacron-reinforced compression molded valve showing failure due to tear formation at the center of coaptation at an equivalent of 0.635 years.

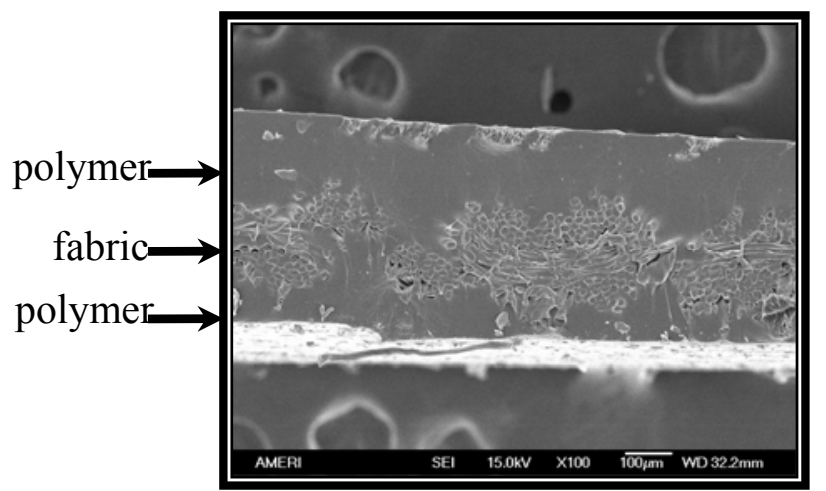

Figure 44. SEM of cross section of compression-molded leaflets. Note how the reinforcement fabric is crushed between two layers of polymer.

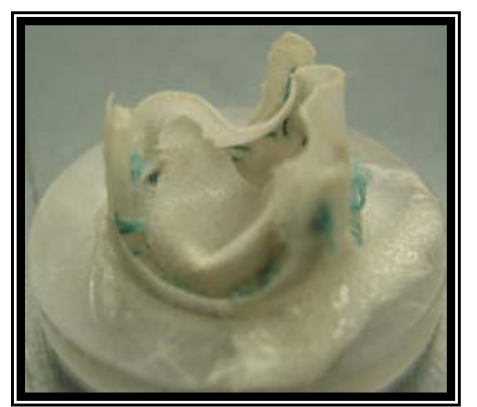

Figure 45. Dacron reinforced hybrid valve showing failure due to tear initiation at the heat sealed joint at an equivalent of 1.5 years. 


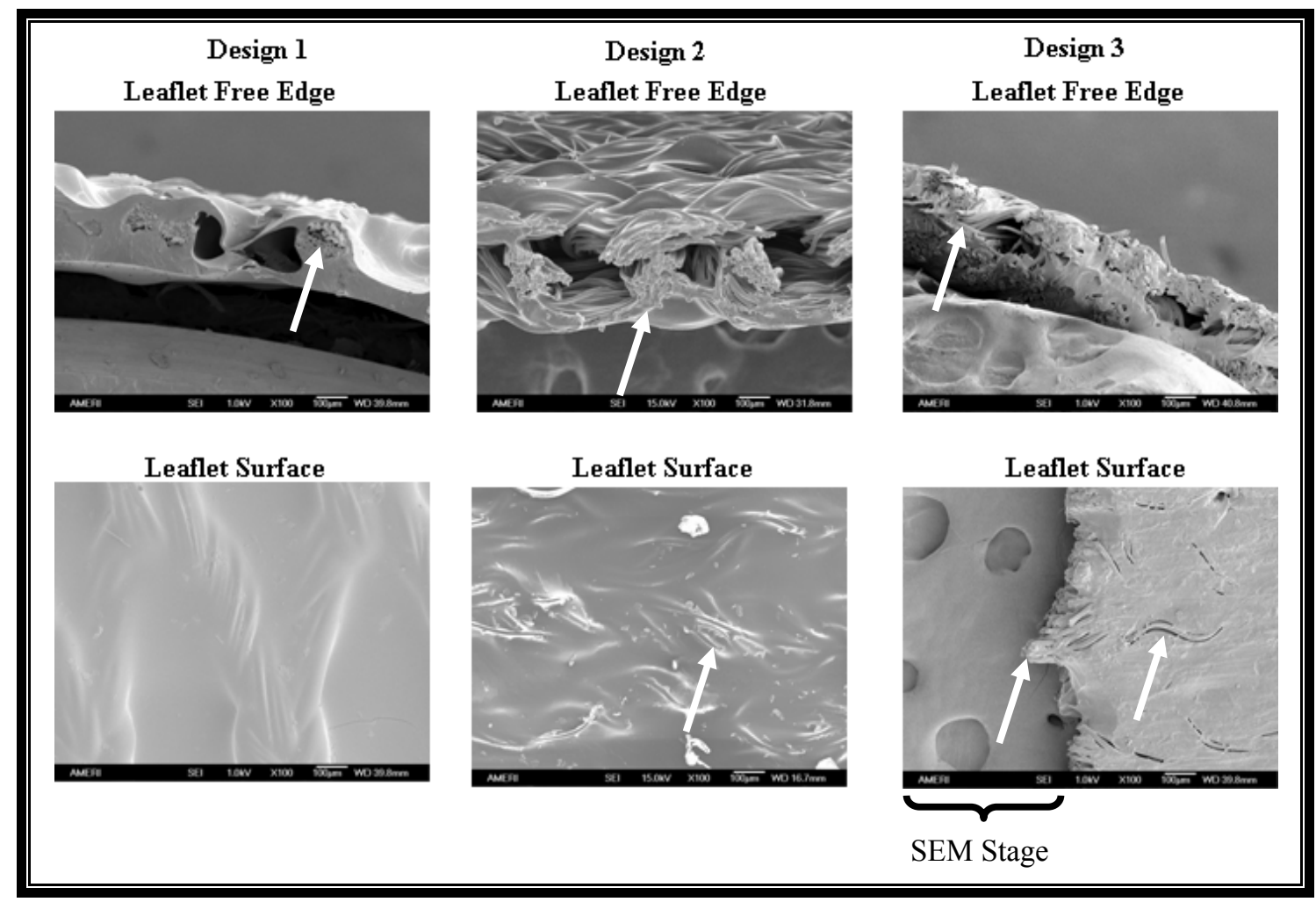

Figure 46. SEM images of the leaflet free edge and aortic surface of SIBS valve designs 1, 2, and 3. Samples were excised from freshly manufactured valves. The white arrows denote exposed polyester fibers. At the leaflet free edge, exposed fibers are expected due to how the edge is cut and exposed, but the leaflet surface should not have exposed fibers.

\subsubsection{SEM}

In vivo studies carried out on the three valve designs showed an inflammatory reaction due to Designs 2 and 3, but none due to Design 1. So the question one might ask is: why did the second two designs cause an inflammatory response but the first did not? Dacron is known to elicit an inflammatory response in vivo [71], but the assumption was that if it was sufficiently coated, it would not do this. The problem arose when the Dacron was not sufficiently coated. It can be seen from the SEM images (Figure 46) that both the cross section and leaflet surface of Design 1 have good fabric covering when compared with Designs 2 and 3. The present manufacturing technique for the valve 
means that there is going to be uncoated Dacron at the free edge, but in Designs 2 and 3, uncoated Dacron fibers escape on both the free edge and the surface. These uncoated fibers could have triggered the immune response witnessed.

An even more noticeable finding was seen after fatigue of the samples: The underlying Dacron from Designs 2 and 3 appears to have unraveled at the free edge and crept to the surface along the body of the leaflet. This increase in fiber exposure due to fatigue could have further contributed to the inflammatory response seen in vivo.

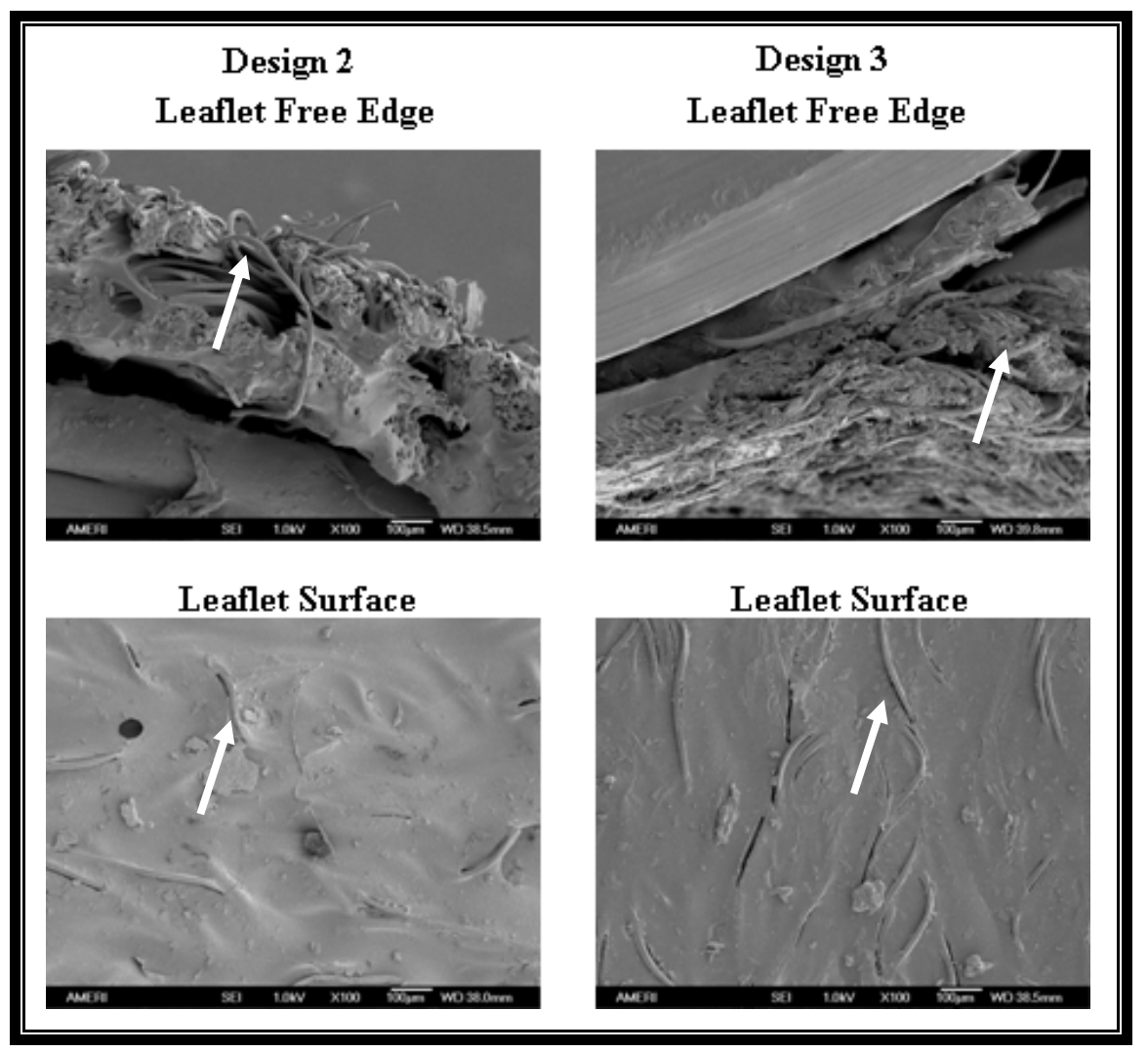

Figure 47. SEM images of the leaflet free edge and aortic surface of SIBS valve designs 2, and 3. Samples were excised from valves subjected to accelerated fatigue testing for an equivalent of 1.25 years. The white arrows denote exposed polyester fibers. Note the increase in fiber exposure on both leaflet surfaces and the fraying of fibers at the free edge. 


\subsection{Design Refinement and Quantification for Improved Durability}

Based on these results, it was determined that the best combination of features for the trileaflet valve included:

- The stiffer, medium profile stent from Design 3

- The cylinder valve design from Designs 2 and 3

- The more dense leaflet reinforcement fabric from Designs 2 and 3

- The thinner leaflet reinforcement fabric from Design 1

- A leaflet manufacturing process that resulted in complete coverage of the underlying reinforcement fabric like what was achieved in Design 1

- The lower styrene content leaflet matrix polymer.

A stronger supporting stent is required to prevent the excessive deformations seen in vivo for the $30 \%$ styrene, high profile stents; the cylinder valve design allows a more secure leaflet-stent attachment technique and a better leaflet curvature in terms of reduced pressure drop; a more dense leaflet reinforcement fabric is needed to provide adequate structural support for the SIBS matrix; the lower styrene content $(8.5 \%)$ leaflet polymer is preferred to prevent polymer cracking and fiber exposure; and a thinner leaflet reinforcement fabric in combination with a new polymer coating technique is preferred to allow adequate coating of the reinforcement with SIBS without resulting in excessive leaflet thickness. It should be noted that the nominal thickness of the Dacron reinforcement is $0.3 \mathrm{~mm}$, while the LARS ${ }^{\circledR}$ reinforcement is only $0.15 \mathrm{~mm}$, and the dipcoated thicknesses are $0.40 \mathrm{~mm}$ and $0.21 \mathrm{~mm}$ respectively.

Based on these results and the preliminary in vitro and in vivo experiments presented by Gallocher, Schoephoerster, and Wang et al. [7, 10, 11, 12, 13, 14], it was 
determined that the preferred reinforcement mesh would be one with a maximum thickness of approximately $0.20 \mathrm{~mm}$ and a density sufficient to prevent the visual exposure of non-reinforced SIBS. The materials chosen for leaflet reinforcement were restricted by what was commercially available. Materials with suitable mechanical and biological properties included polyester (PET) and polypropylene, but only PET fabrics were found that met the above criteria.

\subsubsection{Leaflet Design and Manufacture}

Due to the unsatisfactory results obtained with the dip-coating and compression molding manufacturing techniques from Design 2 and Design 3, a solvent casting procedure was developed to ensure the uniform and complete coating of the reinforcement fabrics. The solvent casting technique in combination with the new fabric reinforcements allowed the production of leaflets whose overall thickness was less than $0.25 \mathrm{~mm}$ (Table 9).

Table 9. Summary of uncoated and coated thicknesses of the dip coated Dacron and all samples manufactured by solvent casting. The precision of the digital calipers is provided.

\begin{tabular}{|l|c|c|c||}
\hline $\begin{array}{c}\text { Reinforcement } \\
\text { Fabric }\end{array}$ & $\begin{array}{c}\text { Uncoated } \\
\text { Thickness } \\
( \pm 0.005 \mathrm{~mm})\end{array}$ & $\begin{array}{c}\text { Coated } \\
\text { Thickness } \\
( \pm 0.005 \mathrm{~mm})\end{array}$ & $\begin{array}{c}\text { Leaflet } \\
\text { Manufacturing } \\
\text { Technique }\end{array}$ \\
\hline PET Knit 1 (JSI) & $0.30 \mathrm{~mm}$ & $0.45 \mathrm{~mm}$ & Dip-coating \\
\hline PET Weave & $0.15 \mathrm{~mm}$ & $0.20 \mathrm{~mm}$ & Solvent casting \\
\hline PET Mesh 3 (NZ 11) & $0.21 \mathrm{~mm}$ & $0.26 \mathrm{~mm}$ & Solvent casting \\
\hline PET Mesh 1 (XA 47) & $0.16 \mathrm{~mm}$ & $0.20 \mathrm{~mm}$ & Solvent casting \\
\hline PET Knit 2 (BARD) & $0.15 \mathrm{~mm}$ & $0.20 \mathrm{~mm}$ & Solvent casting \\
\hline
\end{tabular}

\subsubsection{Experimental Evaluation}

The material formulations presented in Table 9 were used for the production of trileaflet valves, and their material properties were assessed according to the procedures 
outlined in Section 4.2 to determine how their properties impact valve function and durability. The valves were of the preferred cylinder-valve design, and the leaflets were mounted on the medium profile, $48.5 \%$ styrene stent. Even though the dip-coated, PET knit type 2 (JSI) had been eliminated as a prospective leaflet material, it was still tested in this configuration because: 1) In terms of durability, it has been the most successful reinforcement to date when mounted on a high-profile, flexible stent, so it is a good gauge of ideal durability; and 2) by mounting it on a less flexible stent, the impact of changing stent design could be assessed.

\subsubsection{1. $\quad$ Tensile Testing}

Tensile tests were carried out on the reinforced leaflet materials, the isotropic polymer used in leaflet fabrication, and the isotropic polymers used in the stent. The reinforced leaflet materials were tested primarily along their circumferential and radial directions, but a selection were also tested at $45^{\circ}$ between the circumferential and radial directions.

As can be seen from the stress-strain plots (Figure 48 - Figure 51), a vast array of material properties existed for the fiber-reinforced leaflet materials, where differences between materials became more prevalent at the higher strain levels. For the polyester weave and the XA-47 polyester mesh materials, the properties in the radial and circumferential directions were identical due to the make-up of the fabric, whose properties were the same in the orthotropic directions. In both of these reinforcement materials, the fabric was oriented so that their fibers ran $45^{\circ}$ between the radial and circumferential directions, so it was in this orientation that the higher stiffness in the material existed. This phenomenon becomes more evident in Table 10, where the 
engineering and true Young's moduli are presented for all three directions. For these materials, the properties in the circumferential and radial directions were predominated by the matrix properties (Figure 52); however, their overall properties did deviate from that of the underlying polymer due to the presence of the fibers (Figure 53). It is well known that for composites in which the applied stress is parallel to the direction of the reinforcement fibers, the modulus of elasticity approaches that of the fibers as the fiber volume fraction increases. If the applied stress is perpendicular to the fibers, the modulus of elasticity tends to mimic that of the matrix material [66], so when the applied stress is between those two orientations, the Young's modulus falls between that of the fibers and the polymer matrix.

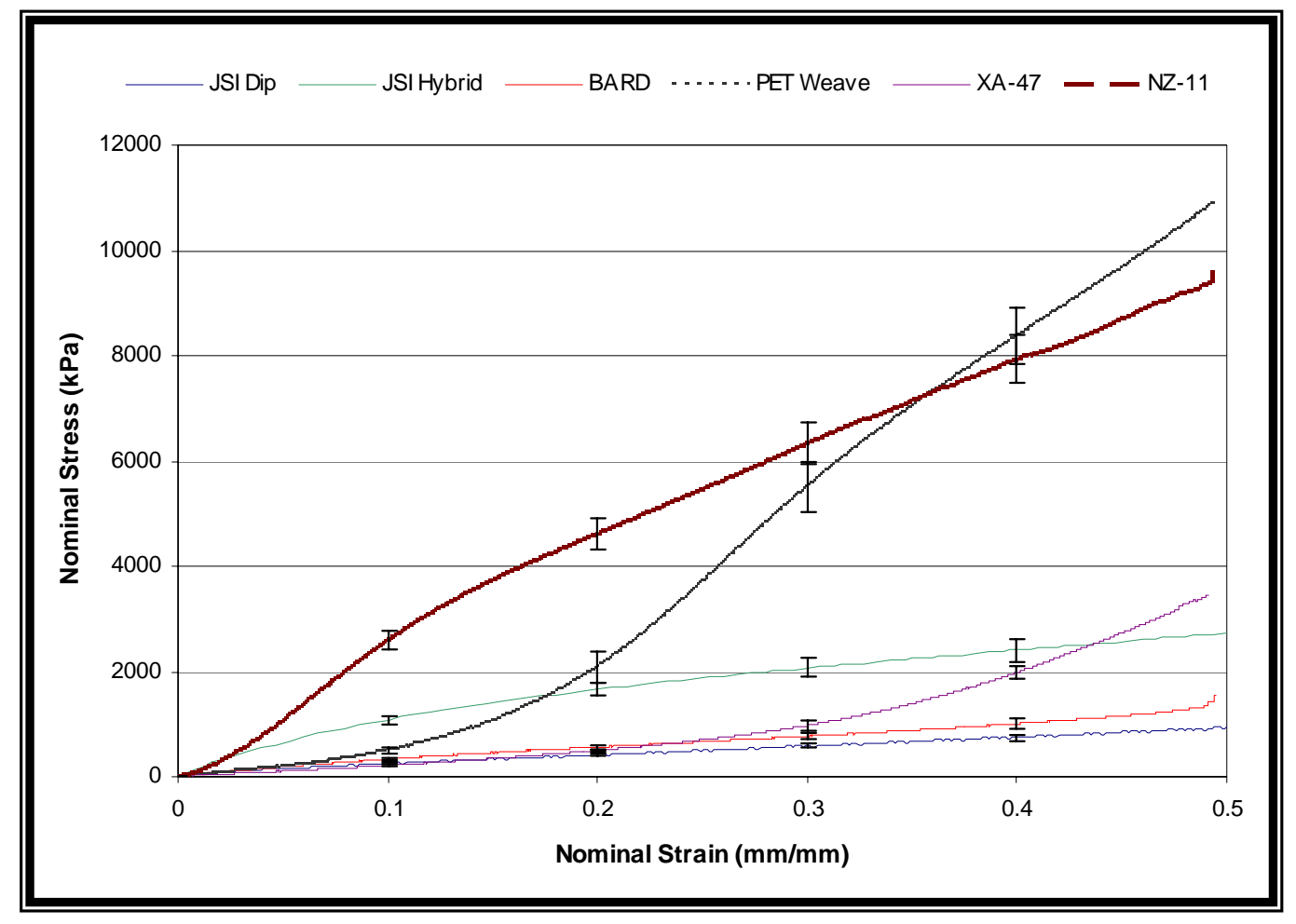

Figure 48. Circumferential stress versus strain curve for all fiber-reinforced leaflet materials $\mathrm{N}=5$ for all samples. 


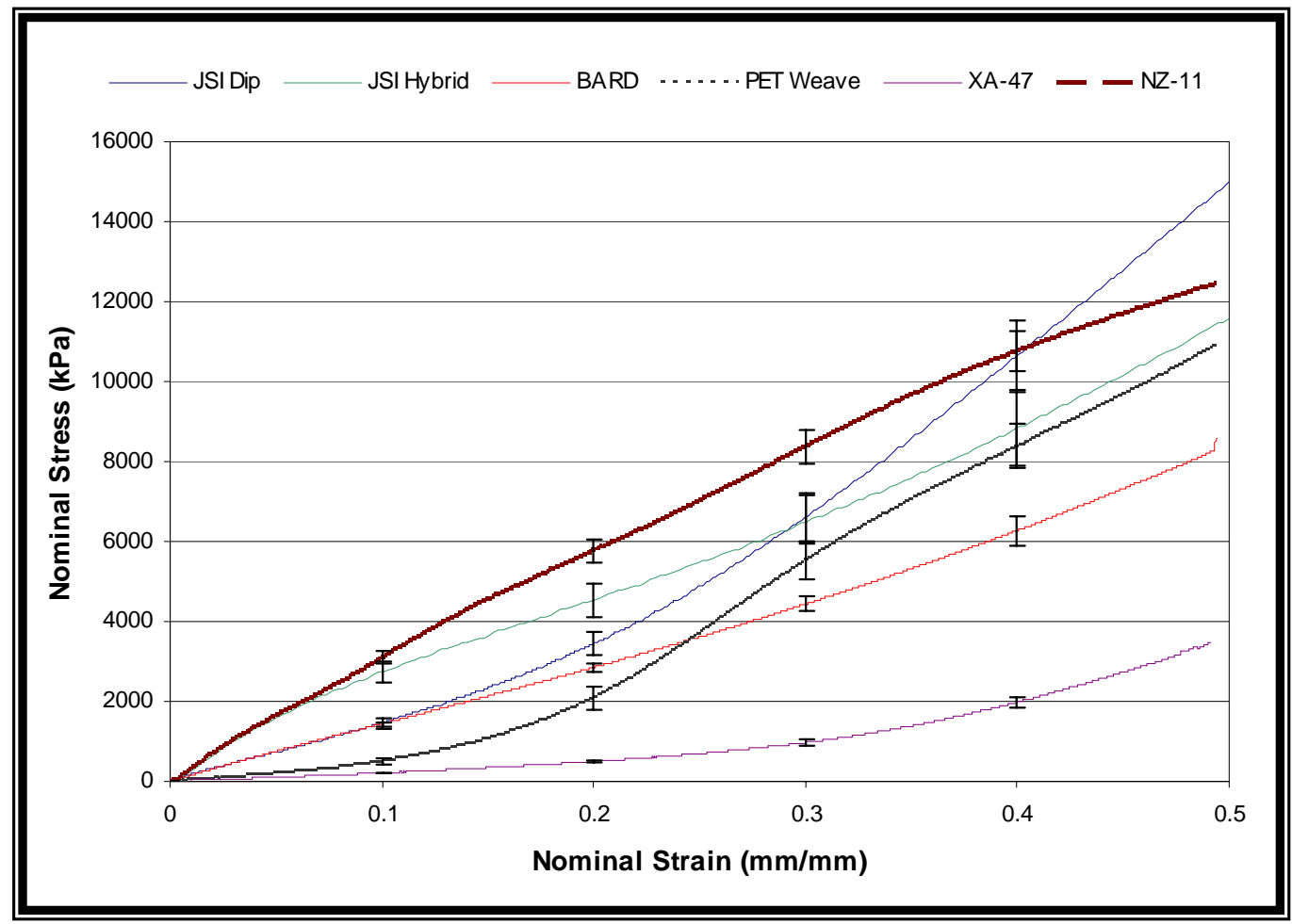

Figure 49. Radial stress versus strain curves for all fiber-reinforced leaflet materials. $\mathrm{N}=5$ for all samples.

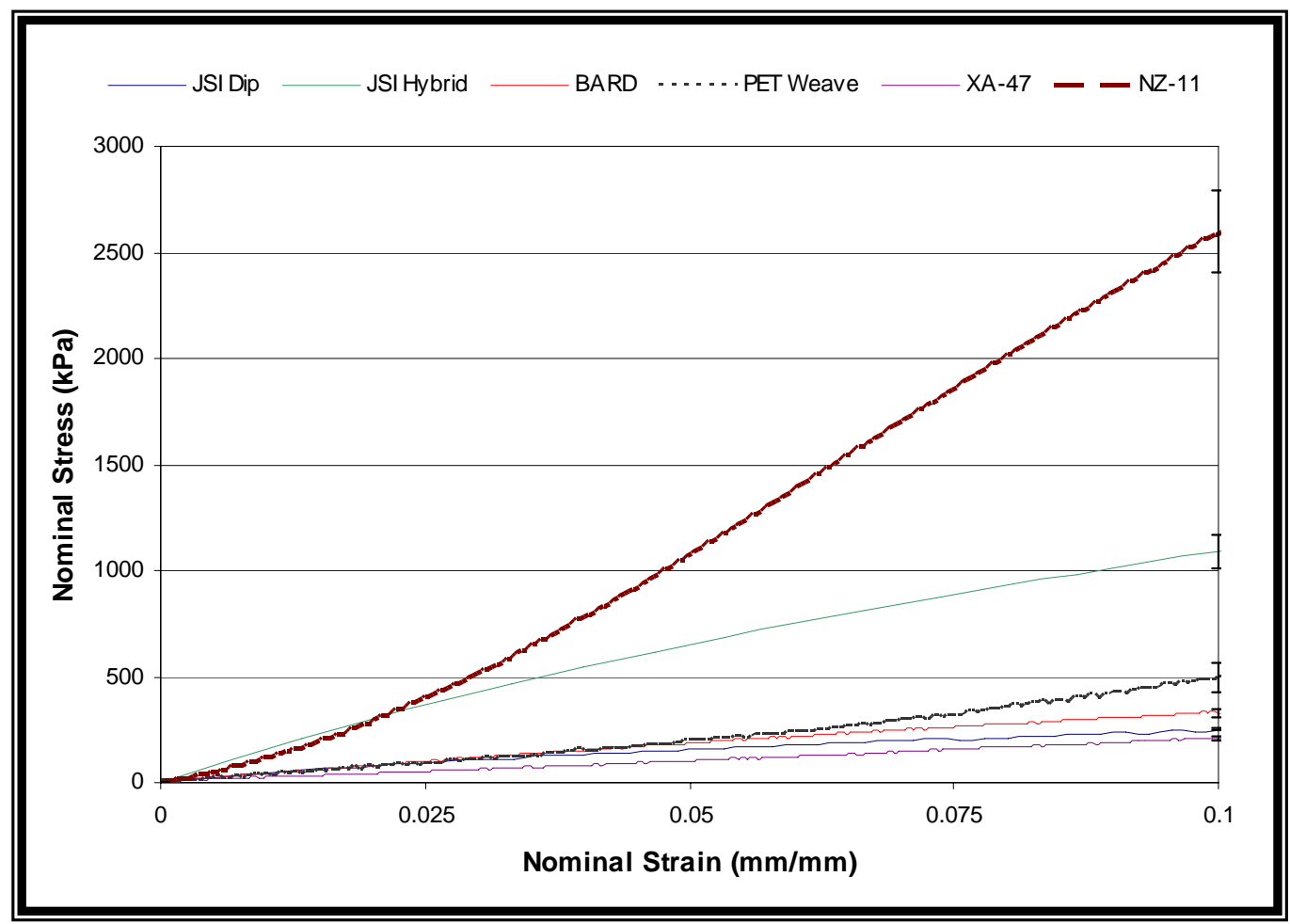

Figure 50. Circumferential stress versus strain curves for all fiber-reinforced materials up to $10 \%$ strain. N $=5$ for all samples. 


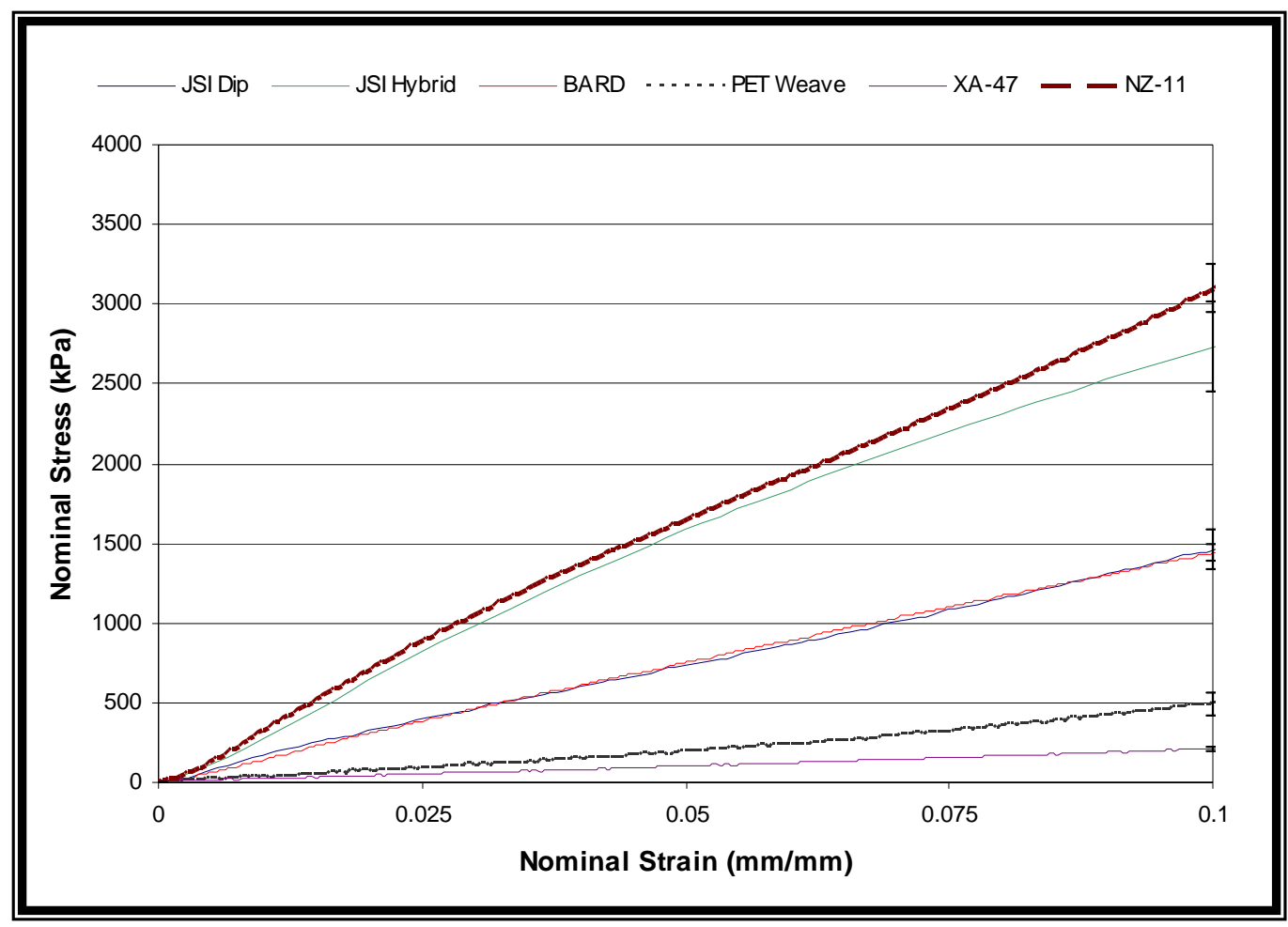

Figure 51. Radial stress versus strain curves for all fiber-reinforced materials up to $10 \%$ strain. $N=5$ for all samples.

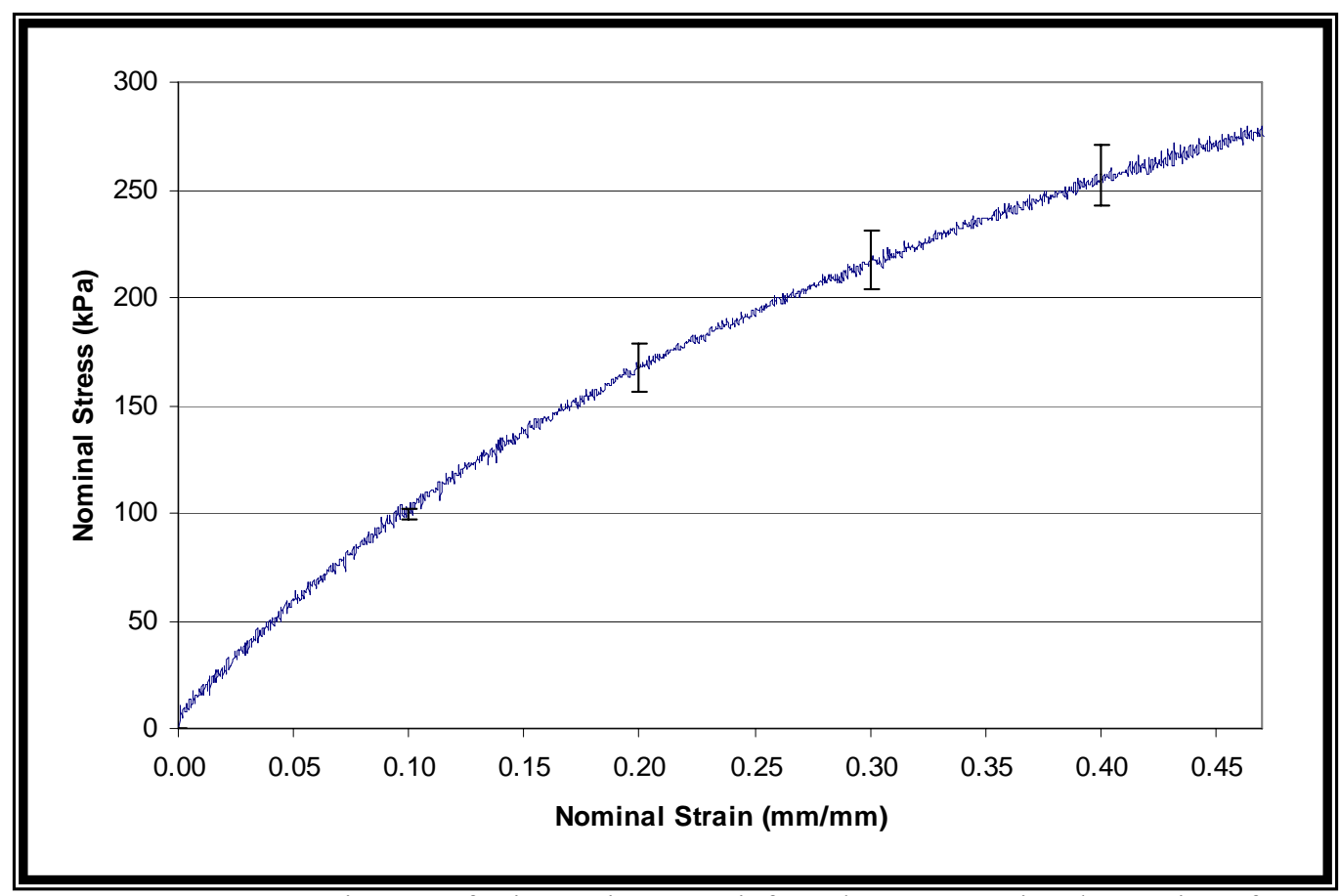

Figure 52. Stress versus strain curve for isotropic, non-reinforced $8.5 \%$ SIBS i.e. the version of SIBS used in leaflet production. $\mathrm{N}=5$ for all samples. 


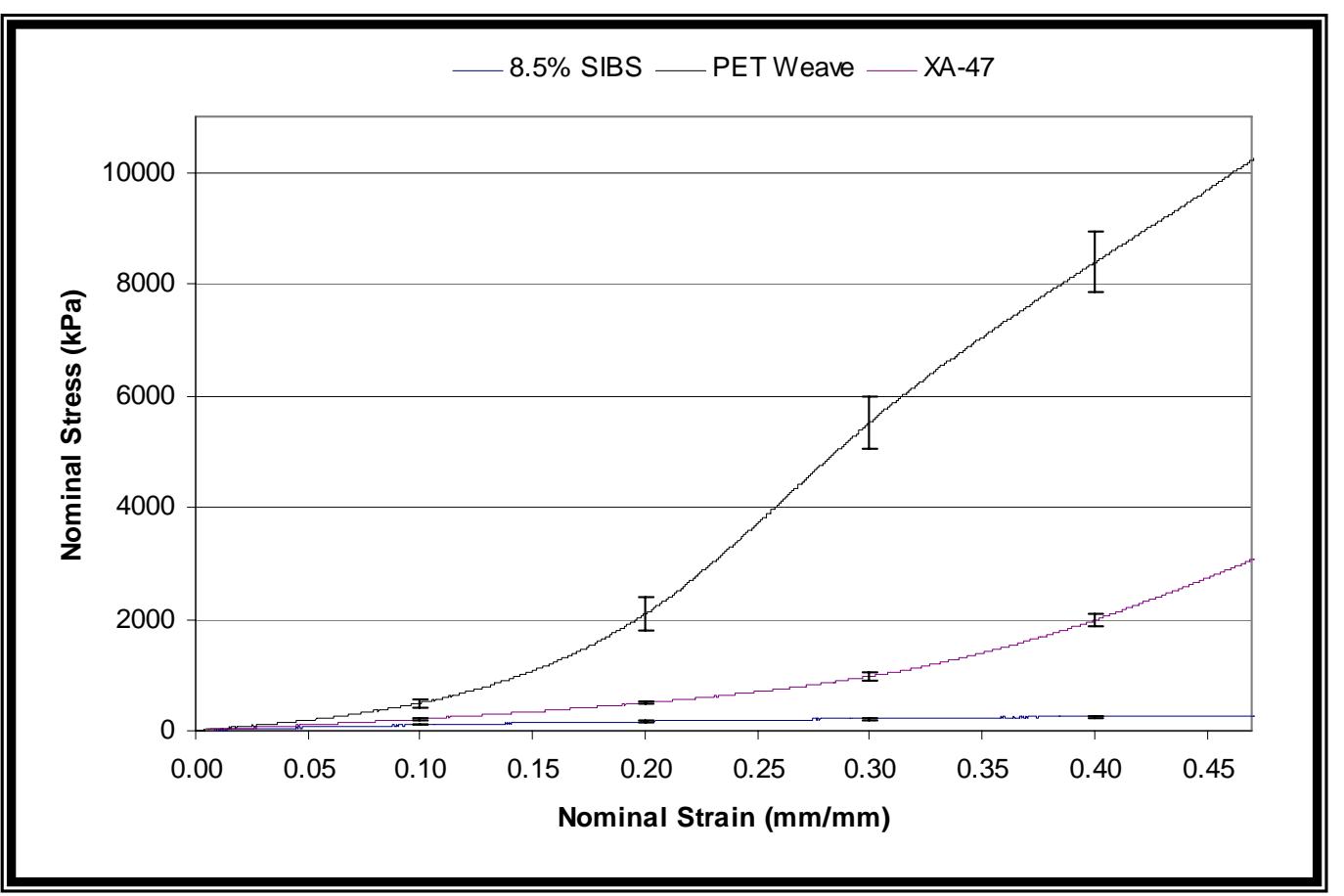

Figure 53. Stress versus strain curve for isotropic, non-reinforced 8.5\% SIBS, PET weave-reinforced $8.5 \%$ SIBS, and XA-47 - reinforced $8.5 \%$ SIBS. $\mathrm{N}=5$ for all samples.

The stress-strain curves for the two polymers used to fabricate the valve stent are provided in Figure 54. By increasing the styrene percent from 30 to $48.5 \%$, a large change in the material response can be seen. For the $30 \%$ styrene SIBS, the material yields at a stress $\left(\sigma_{\mathrm{y}}\right)$ of approximately $2,900 \mathrm{kPa}$ and a $6 \%$ strain $\left(\varepsilon_{\mathrm{y}}\right)$, whereas, the $48.5 \%$ styrene SIBS has a $\sigma_{y}$ of $10,585 \mathrm{kPa}$ and a $\varepsilon_{\mathrm{y}}$ of $4 \%$. There is a 323 MPA difference in the Young's Moduli of the two formulations (Table 10). An ANOVA was run comparing the engineering and true Young's moduli of these materials, and they were found to be significantly different with $p=0.001$ for both values.

It is apparent from the stress-strain curves that not all materials exhibited a linear relationship. In fact, many displayed a similar tendency to the natural valve properties (Figure 40), where an increase in the slope of the curve occurred at higher strain levels. 
Nevertheless, Young's moduli for all materials were determined based on the initial slope of the stress-strain curve from 0 to $5 \%$ strain (Table 10), and an ANOVA and subsequent post hoc test was carried out to assess for significant difference between materials. When comparing the properties for the leaflet materials (including the $8.5 \%$ SIBS) in the circumferential direction, the NZ-11 mesh and the JSI hybrid formulations were found to be significantly different from each other and all other leaflet formulations. The $8.5 \%$ SIBS, XA-47 mesh, and dip-coated JSI knit were not found to be significantly different $(\mathrm{p}=0.079)$, as were the XA-47 mesh, dip-coated JSI knit, BARD knit, and PET weave ( $p$ $=0.078)$. When an ANOVA and subsequent post hoc test was run for the radial samples, the $8.5 \%$ SIBS, XA-47 mesh, and PET weave were not significantly different $(\mathrm{p}=0.169)$, the dip-coated JSI knit and BARD knit were not significantly different $(p=0.984)$, and the hybrid JSI knit and NZ-11 mesh were not significantly different $(\mathrm{p}=0.916)$.

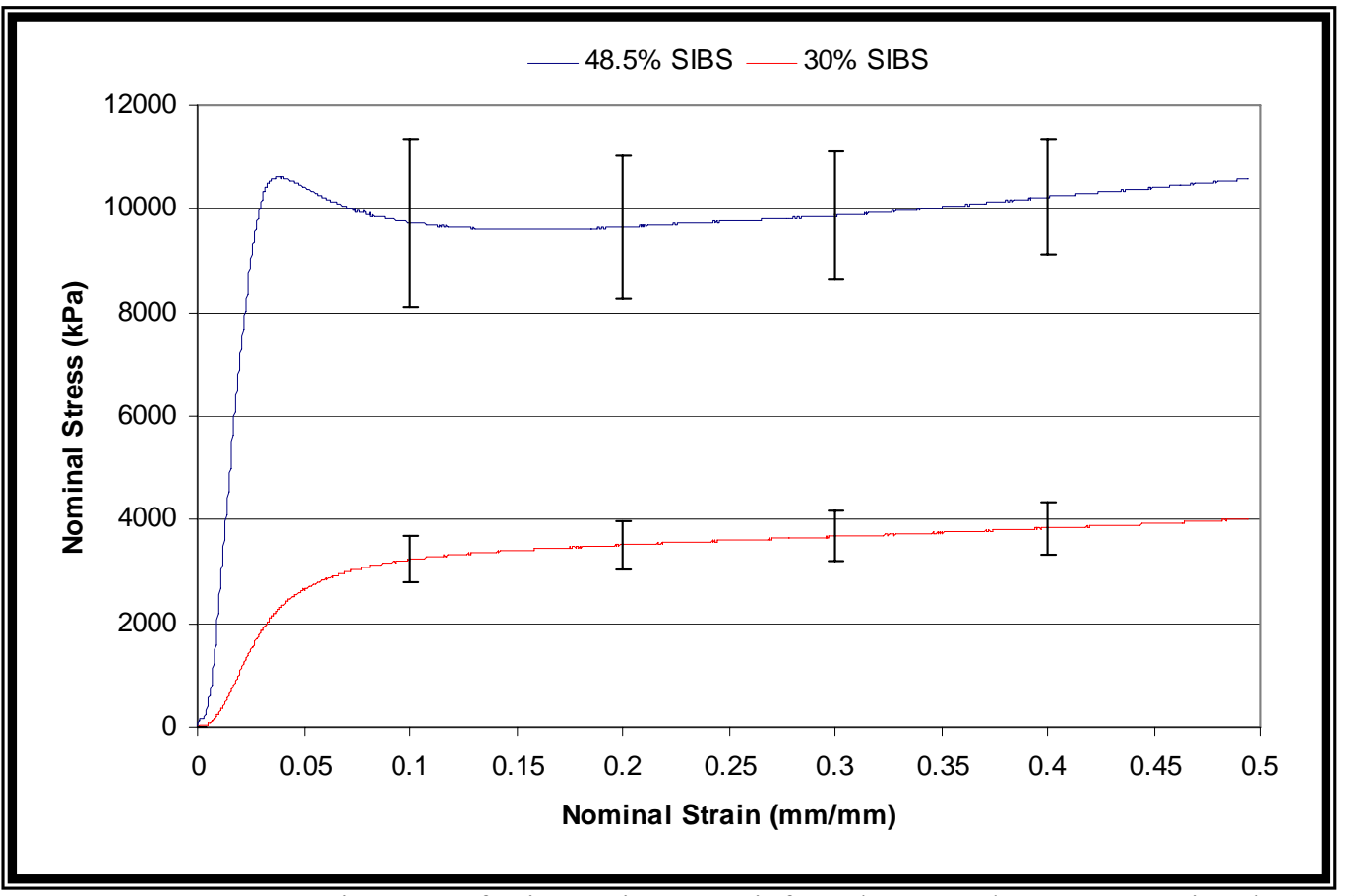

Figure 54. Stress versus strain curves for isotropic, non-reinforced $30 \%$ and $48.5 \%$ SIBS i.e. the versions of SIBS used stent production. $\mathrm{N}=5$ for all samples. 
Table 10. Engineering and true Young's moduli for fiber-reinforced leaflet materials, isotropic leaflet material, and isotropic stent materials in the circumferential direction, radial direction, and $45^{\circ}$ in between.

\begin{tabular}{|c|c|c|c|c|c|c|}
\hline \multirow{2}{*}{ Material } & \multicolumn{2}{|c|}{ Circumferential } & \multicolumn{2}{c|}{ Radial } & \multicolumn{2}{c|}{$\mathbf{4 5}^{\circ}$} \\
\cline { 2 - 7 } & $\mathbf{E}_{\text {eng }}(\mathbf{k P a})$ & $\mathbf{E}_{\text {true }}(\mathbf{k P a})$ & $\mathbf{E}_{\text {eng }}(\mathbf{k P a})$ & $\mathbf{E}_{\text {true }}(\mathbf{k P a})$ & $\mathbf{E}_{\text {eng }}(\mathbf{k P a})$ & $\mathbf{E}_{\text {true }}(\mathbf{k P a})$ \\
\hline BARD & $3669^{\#}$ & 3953 & $15351^{\circledR}$ & 16508 & 5835 & 6272 \\
\hline PET Weave & $3797^{\#}$ & 4055 & $3797^{+}$ & 4055 & 79312 & 84921 \\
\hline JSI Dip & $2973^{*, \#}$ & 3220 & $14504^{\circledR}$ & 15622 & & \\
\hline JSI Hybrid & $12651^{*}$ & 13699 & $33034^{+}$ & 35502 & & \\
\hline XA-47 & $1884^{*}, \#$ & 2034 & $1884^{+}$ & 2034 & 32214 & 34525 \\
\hline NZ-11 & $21837^{*}$ & 23420 & $34229^{\circledR}$ & 36798 & 13399 & 14388 \\
\hline $8.5 \%$ SIBS & $1064^{*}$ & 1151 & $1064^{+}$ & 1151 & 1064 & 1151 \\
\hline $30 \%$ SIBS & $62429^{+}$ & 66935 & $62429^{*}$ & 66935 & 62429 & 66935 \\
\hline $48.5 \%$ SIBS & 385372 & 395969 & 385372 & 395969 & 385372 & 395969 \\
\hline
\end{tabular}

Note: $\mathrm{N}=5$; and *,\#,+, @, \& denote groups that are not significantly different, i.e. $\mathrm{p}<0.05$

\subsubsection{2. $\quad \underline{\text { Hydrodynamics }}$}

The fiber-reinforced leaflet materials whose mechanical properties were assessed in Section 5.2.2.1 were used to fabricate valves mounted on a $48.5 \%$, medium profile stent, and these valves were subjected to both hydrodynamic and fatigue testing. For each valve, the measurement was repeated three times, and the results are represented as averages and standard deviations. By taking repetitive measures, the variations in data acquisition can be accounted for.

The transvalvular pressure drop results presented in Figure 55 appear to be divided into three subsets, where the dip-coated JSI leaflets resulted in a consistently higher pressure drop, the hybrid JSI and PET weave leaflets resulted in a medium pressure drop, and the Magna, BARD, XA-47, and NZ-11 leaflets had the lowest pressure drop.

In terms of percent regurgitation (Figure 56), the data again appeared to be divided into three subsets, where the NZ-11 reinforced valve exhibited the highest 
regurgitation, the dip-coated JSI (48.5\% styrene stent) and XA-47 valves showed regurgitation in the medium range, and the remaining valves had an average regurgitation under $5 \%$

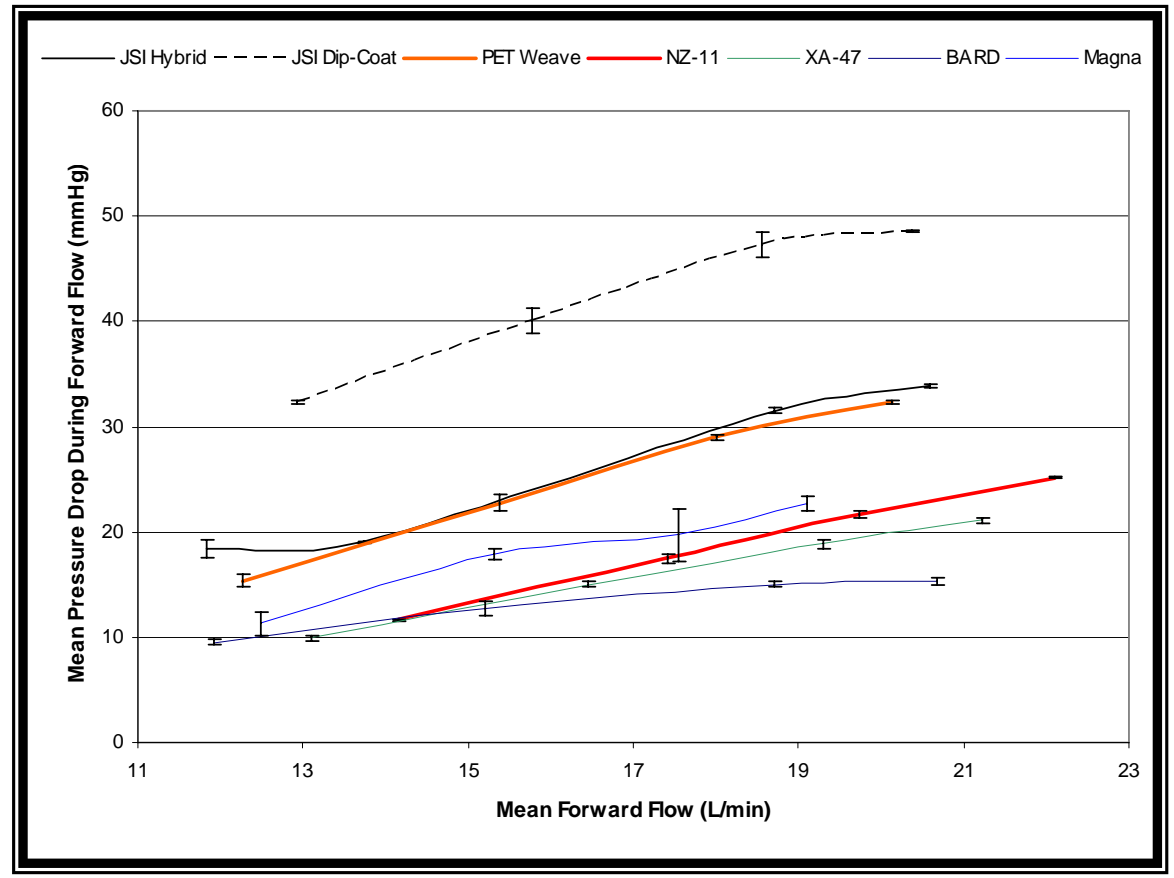

Figure 55. Transvalvular pressure drop during forward flow for all prototype valves mounted on the $48.5 \%$ styrene stent. The results for the $19 \mathrm{~mm}$ TAD Magna ${ }^{\circledR}$ valve are provided for comparison. Error bars denote standard deviation between the three repeated measures.

A summary of the pressure drop, percentage regurgitation, effective orifice area, and leaflet thickness is provided for all of the valves that have been tested (Table 11). The transvalvular pressure drop and percent regurgitation values were averaged over all cardiac output - heart rate combinations, while the values for $A_{E O}$ were calculated at a heart rate of $70 \mathrm{BPM}$ and a cardiac output of $5.6 \mathrm{~L} / \mathrm{min}$. From this summary, it is apparent that the St. Jude bileaflet valve had a considerably lower pressure drop than all other valves, and the Magna valve had the lowest percentage regurgitation. An ANOVA 
and subsequent post hoc test was run to assess for significance between the pressure drop and regurgitation results.

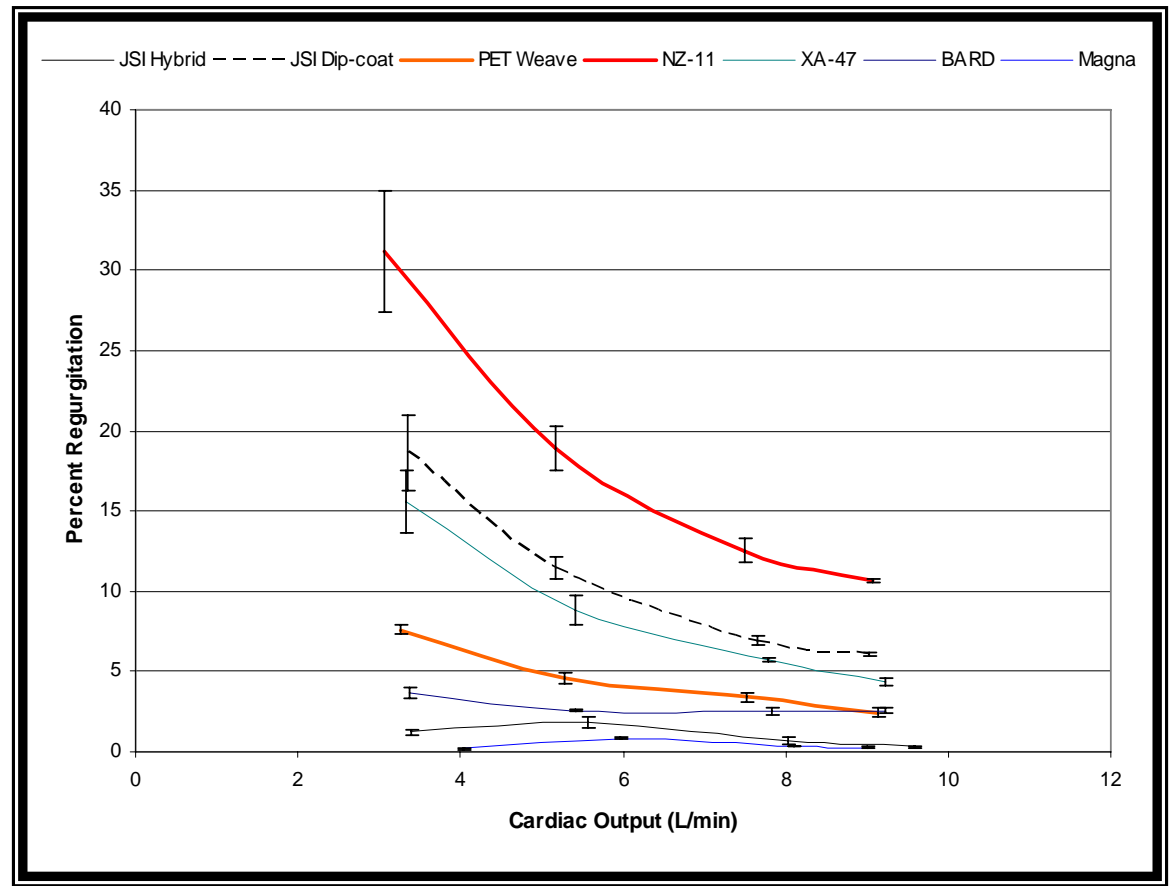

Figure 56. Percentage regurgitation flow for all prototype valves mounted on the $48.5 \%$ styrene stent. The results for the $19 \mathrm{~mm}$ TAD Magna ${ }^{\circledR}$ valve are provided for comparison. Error bars denote standard deviation between the three repeated measures.

Tukey's post hoc tests comparing transvalvular pressure drops between valves determined that: the pressure drops for the Magna and XA-47 valves were not significantly different $(p=0.195)$, the pressure drops for the Magna and NZ-11 valves were not significantly different $(\mathrm{p}=0.793)$, the pressure drops for the PET weave and hybrid JSI valves were not significantly different $(\mathrm{p}=0.937)$, the pressure drops for the dip-coated JSI (48.5\% styrene stent) and LARS ${ }^{\circledR}$ valves were not significantly different $(p=0.956)$, and all other combinations of valves not mentioned are significantly different from each other. A summary of the significant results is provided in Table 11. 
Table 11. Summary of mean transvalvular pressure drop during forward flow, percentage regurgitation, effective orifice area $\left(\mathrm{A}_{\mathrm{EO}}\right)$, and leaflet thickness for all valves subjected to hydrodynamic testing. Values for the pressure and regurgitation were averaged over all cardiac output and heart rate combinations, and $\mathrm{A}_{\mathrm{EO}}$ was measured at $70 \mathrm{BPM}, 5.6 \mathrm{~L} / \mathrm{min}$.

\begin{tabular}{|c|c|c|c|c|c|}
\hline \multicolumn{2}{|r|}{ Valve } & $\begin{array}{l}\text { Mean } \Delta P \text { during } \\
\text { forward flow }\end{array}$ & $\underset{\%}{\text { Regurgitation }}$ & $\begin{array}{c}\mathrm{A}_{\mathrm{EO}} \\
\left(\mathrm{cm}^{2}\right)\end{array}$ & $\begin{array}{l}\text { Leaflet } \\
\text { Thickness }\end{array}$ \\
\hline 1 & $\begin{array}{c}\text { LARS 3-Leaflet Valve, } \\
\text { Implant } 1\end{array}$ & $42.85^{@}$ & $7.35^{+, @)}$ & 0.90 & 0.21 \\
\hline 2 & $\begin{array}{l}\text { JSI: High Profile, } 30 \% \\
\text { Styrene, Dip, Implant } 2\end{array}$ & 38.02 & $4.23^{\#,+}$ & 1.03 & 0.45 \\
\hline 3 & $\begin{array}{c}\text { JSI: Medium Profile, } \\
\text { 48.5\% Styrene, } \\
\text { Hybrid, Implant } 3\end{array}$ & $25.71^{+}$ & $1.01{ }^{*}$ & 1.15 & 0.30 \\
\hline 4 & $\begin{array}{l}\text { JSI: Medium Profile, } \\
\text { 48.5\% Styrene, Dip }\end{array}$ & $42.05^{@}$ & $10.76^{\&}$ & 0.93 & 0.45 \\
\hline 5 & $\begin{array}{l}\text { PET Weave: Medium } \\
\text { Profile, } 48.5 \% \text { Styrene }\end{array}$ & $24.86^{+}$ & $4.50^{\#,+}$ & 1.21 & 0.20 \\
\hline 6 & $\begin{array}{c}\text { NZ-11: Medium } \\
\text { Profile, 48.5\% Styrene }\end{array}$ & $18.97^{\#}$ & 18.30 & 1.57 & 0.26 \\
\hline 7 & $\begin{array}{c}\text { XA47: Medium Profile, } \\
\text { 48.5\% Styrene }\end{array}$ & $16.22^{*}$ & $8.62^{@, \&}$ & 1.59 & 0.20 \\
\hline 8 & $\begin{array}{l}\text { BARD: Med Profile, } \\
\text { 48.5\% Styrene }\end{array}$ & 13.16 & $2.82^{*, \#}$ & 1.60 & 0.20 \\
\hline 9 & Magna & $17.90^{*, \#}$ & $0.41^{*}$ & 1.42 & NA \\
\hline 10 & St Jude & 0.16 & $3.23^{*, \#}$ & 7.51 & NA \\
\hline
\end{tabular}

Note: , $\mathrm{N}=3$; and *,\#,+, @, \& denote groups that are not significantly different, i.e. $\mathrm{p}<0.05$

Tukey's post hoc tests comparing percentage regurgitation between valves determined that: the Magna, hybrid JSI, BARD, and St. Jude valves were not significantly different $(\mathrm{p}=0.126)$, the BARD, St. Jude, dip-coated JSI $(30 \%$ styrene stent), and PET weave valves were not significantly different $(\mathrm{p}=0.787)$, the dip-coated JSI (30\% styrene stent), PET weave, and LARS ${ }^{\circledR}$ valves were not significantly different $(p=0.059)$, the LARS $\AA$ and XA-47 valves were not significantly different $(p=0.952)$, the XA-47 and dip-coated JSI (48.5\% styrene stent) valves were not significantly different $(p=0.482)$, and all other combinations not mentioned were considered to be significantly different. 
It should be noted at this time that statistical significance does not always imply clinical significance. For example, the dip-coated JSI ( $30 \%$ styrene stent) valve was determined to be significantly different from the hybrid JSI valve in terms of percent regurgitation, even though the difference between the average regurgitation was only $3.22 \%$ (calculated from Table 11). In the clinical setting, this would not be considered a regurgitation difference significant enough to take action, so in reality, these two valves cannot be suggested to be clinically different. If one were to classify the pressure difference as being clinically significant if it were greater than $10.0 \mathrm{mmHg}$ and the regurgitation difference to be clinically significant if it were more than $5 \%$, then a broader tolerance range could be provided for assessment of significant difference.

Under all conditions of flow, the St. Jude bileaflet valve had a pressure gradient that was significantly lower than any other valve (both clinically and statistically). This decreased pressure gradient is a characteristic of this market-leading valve; however, the difference between results from all other valves is accentuated by the fact that this valve has a $25 \mathrm{~mm}$ TAD compared with the $19 \mathrm{~mm}$ TAD for all other valves. A larger TAD means a larger $A_{E O}$, and the $A_{E O}$ is inversely proportional to the square root of the transvalvular pressure drop (refer to Equation 1). As a result, one can conclude that if a $19 \mathrm{~mm}$ TAD St. Jude valve were available for comparison, its pressure drop would not be as significantly different from the prototype valves as what is shown here. The minimum performance for a $19 \mathrm{~mm}$ TAD valve (as stated by ISO 5840:2004) requires that $\mathrm{A}_{\mathrm{EO}} \geq$ 0.70 , and all prototype valves fulfilled this requirement (Table 11).

When manufacturing prosthetic trileaflet heart valves, it is assumed that they function like the natural heart valve and replicate the same opening and closing 
characteristics, but according to Thubrikar et al. [75], bioprosthetic leaflets and synthetic polymer leaflets that have an increased stiffness when compared with the natural valve leaflet result in an increased transvalvular pressure drop during systole. The natural heart valve includes a ground substance of glycosaminoglycans that allow movement of collagen fibers with respect to one another. At the same time, the glycosaminoglycans are capable of moving with minimal force, so they provide an environment conducive to bending and shear, thereby allowing valve opening at transvalvular pressures as low as 5 $\mathrm{mmHg}$ [75]. Bioprosthetic valves and polymer trileaflet valves have been said to display elevated bending stresses and an increase in stored bending energy. This promotes valve closure back to its neutral geometry at lower pressure gradients. As a result, an increase in the transvalvular pressure gradient is needed to open these types of valves and keep them open [75].

Rosenhek et al. [76] provided a summary of a selection of prosthetic valve's peak pressure gradient, mean pressure gradient, and $\mathrm{A}_{\mathrm{EO}}$. The pertinent information for a selection of $19 \mathrm{~mm}$ TAD commercial valves has been assembled for comparison with the BARD-reinforced valve mounted on the $48.5 \%$ styrene stent (Table 12). This valve was selected for comparison as it represents the most successful SIBS prototype valve to date in terms of its hydrodynamics. As is apparent from the data, the mean pressure gradient for the BARD valve is lower than that of the selected mechanical and bioprosthetic prostheses, and the $A_{E O}$ is higher. The peak pressure drop, on the other hand, is the highest out of the selection. From this information, it can be assumed that the BARDreinforced SIBS trileaflet valve performs at least as well as the selection of prostheses. 
Table 12. Summary of peak pressure gradient, mean pressure gradient, and $A_{E O}$ for the BARD reinforced valve and a selection of commercially available aortic valve prostheses [76]

\begin{tabular}{||c|c|c|c||}
\hline Valve & $\begin{array}{c}\text { Peak pressure } \\
\text { gradient (mmHg) }\end{array}$ & $\begin{array}{c}\text { Mean pressure } \\
\text { gradient (mmHg) }\end{array}$ & $\left.\mathbf{A}_{\text {EO }} \mathbf{( c m}^{\mathbf{2}}\right)$ \\
\hline BARD & 47.28 & 13.16 & 1.60 \\
\hline $\begin{array}{c}\text { Bjork-Shiley Tilting } \\
\text { Disc }\end{array}$ & 46.0 & 26.67 & 0.94 \\
\hline $\begin{array}{c}\text { Carpentier-Edwards } \\
\text { Stented Bioprosthesis }\end{array}$ & 43.48 & 25.6 & 0.85 \\
\hline $\begin{array}{c}\text { Carpentier-Edwards } \\
\text { Pericardial Stented } \\
\text { Bioprosthesis }\end{array}$ & 32.13 & 24.19 & 1.21 \\
\hline $\begin{array}{c}\text { St. Jude Medical } \\
\text { Bileaflet }\end{array}$ & 35.17 & 18.96 & 1.01 \\
\hline
\end{tabular}

Both leaflet thickness and modulus are proposed to have a large impact on the hydrodynamic function and durability of polymer valves [64]. Leaflets derived from low modulus materials are expected to have good hydrodynamic function as the low modulus materials provide little resistance to opening, thereby allowing a decreased transvalvular pressure drop. Low modulus materials, however, are subject to larger strains than higher modulus materials exposed to the same loading conditions. This strain accumulation results in decreased durability for those materials, so in terms of durability, a higher modulus material that results in a reduction in strain accumulation is more favorable. These higher modulus, stiffer materials are believed to restrict valve opening and are less likely to close fully, so a stiffer leaflet material is predicted to result in less favorable hydrodynamic function. These conflicting phenomena have to, therefore, be considered when designing a polymer valve.

According to Bernacca et al. [65], leaflet thickness is a better predictor of hydrodynamic function than modulus. From a theoretical point of view [65], the bending stiffness of a material is proportional to $\mathrm{t}^{3} \mathrm{E}$, where $\mathrm{t}$ is the thickness of the material and $\mathrm{E}$ is the Young's modulus. Stiffness is then proportional to the cube of the thickness, 
showing the higher dependence on thickness than material modulus. Bernacca evaluated this theory with polyurethane trileaflet valves, where he varied the leaflet thickness from $62-238 \mu \mathrm{m}$, and the Young's modulus from 5.0 - 32.5 MPa. What he found was that leaflet thickness had a direct correlation to transvalvular pressure drop, while leaflet modulus appeared to have no significant impact on hydrodynamic function.

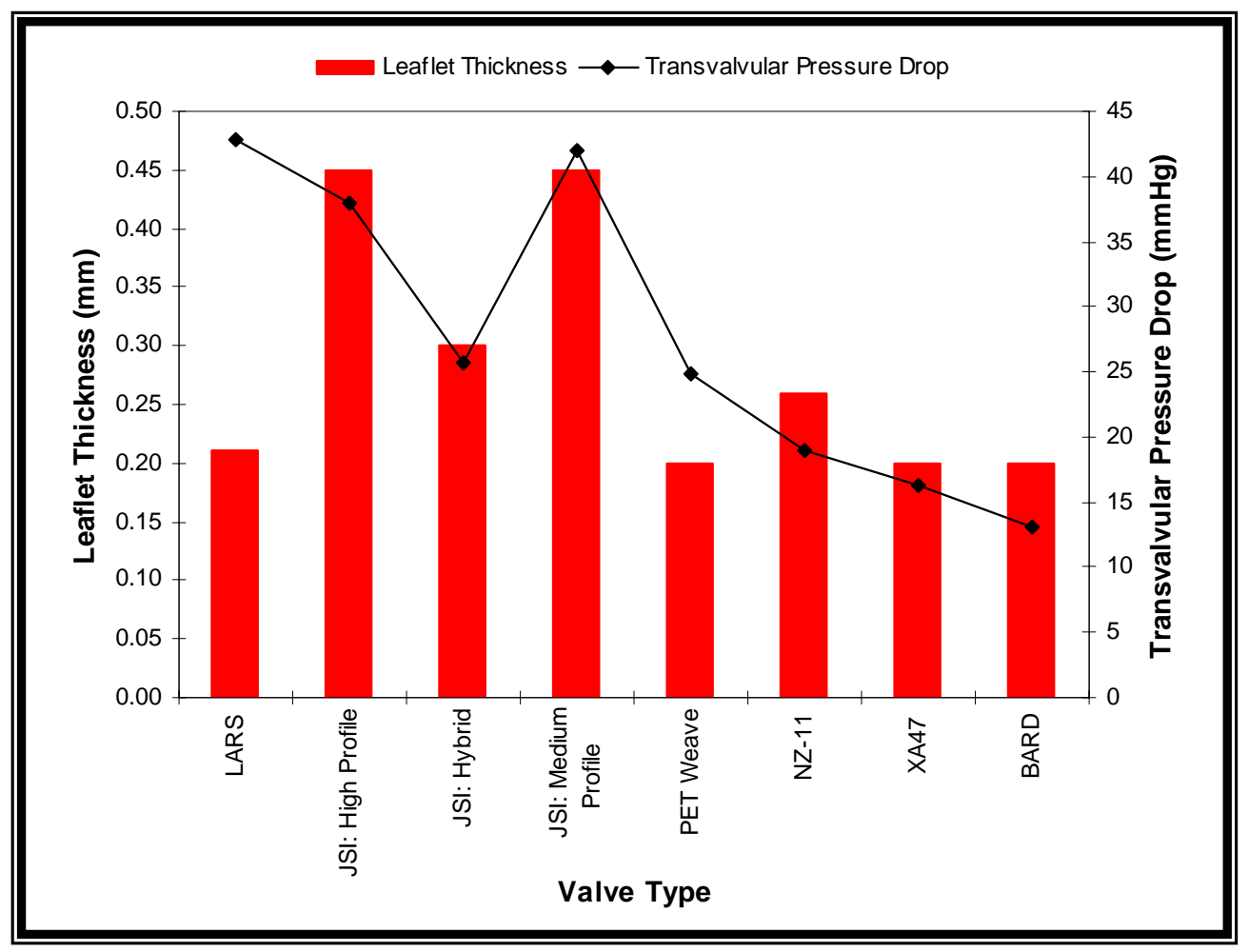

Figure 57. Leaflet thickness versus mean transvalvular pressure drop for all prototype valves.

The dependence of leaflet thickness on pressure drop was examined for all prototype SIBS valves. A strong correlation between the two was seen, as is apparent in Figure 57, but some deviations were evident. The issue with assessing the dependence of leaflet thickness on pressure drop for the SIBS valves is that not all valve designs were equivalent. The LARS ${ }^{\circledR}$ design had a completely different geometry, and many of the 
valves had different fiber reinforcements. While the LARS ${ }^{\circledR}$ valve had a thickness of $0.20 \mathrm{~mm}$, its leaflet geometry prevented the leaflets from opening completely to allow a cylindrical orifice and reduced pressure drop. The cylinder valve designs, on the other hand, did allow this circular orifice upon opening. From this, one can conclude that the design of the leaflets had a greater impact on pressure drop than the leaflet thickness did. As far as the cylinder valves are concerned, a general trend did exist, where the leaflet thickness was inversely proportional to the transvalvular pressure drop. Even though varying leaflet reinforcements were compared, one can conclude that thickness is still an important factor.

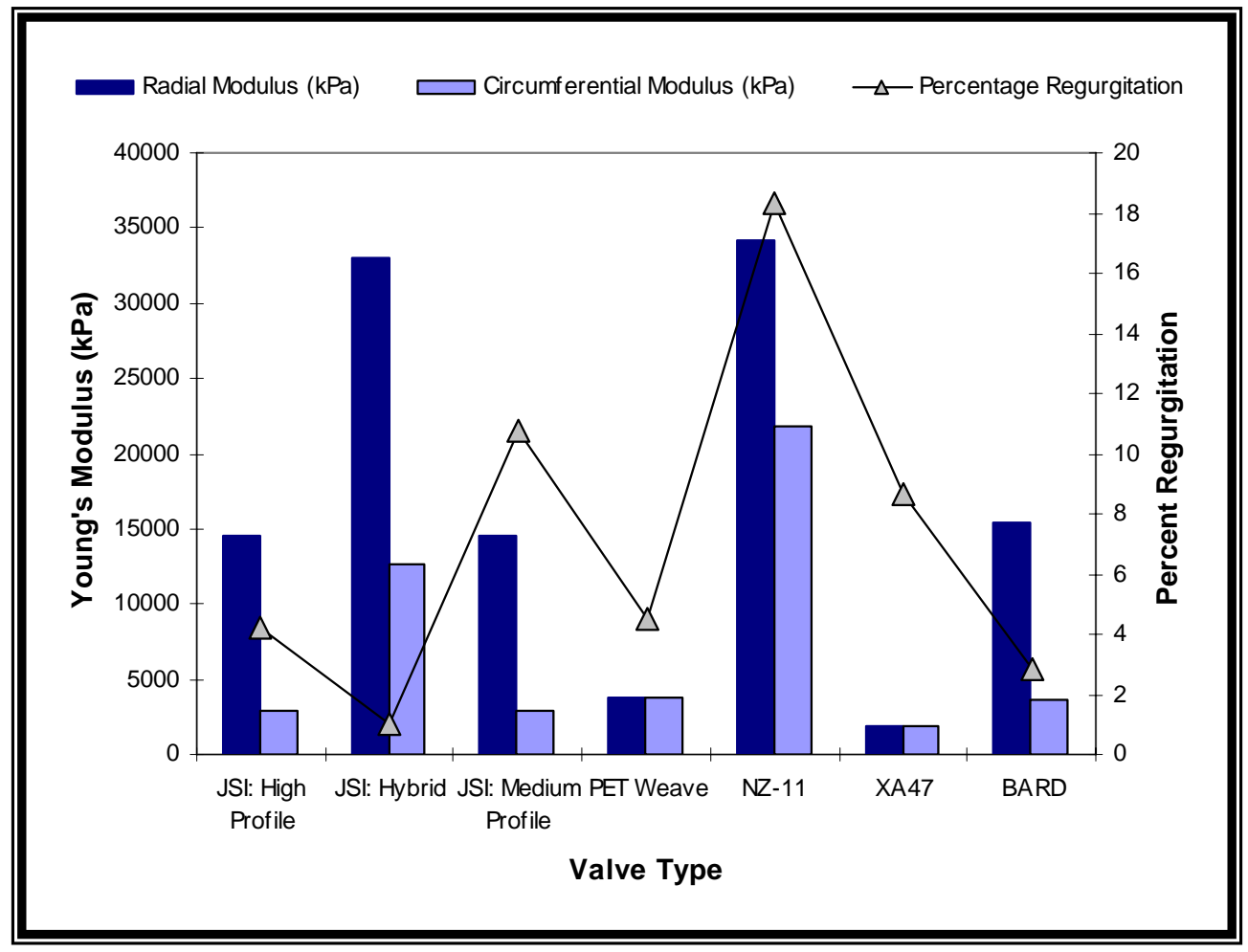

Figure 58. Circumferential and radial Young's modulus versus mean percent regurgitation for all prototype valves 
The dependence of leaflet modulus on valve hydrodynamics was assessed for all SIBS cylinder valves. It is apparent that no correlation existed for either regurgitation or pressure drop (Figure 58 and Figure 59 respectively). Due to the fact that the SIBS valve leaflets are composite structures, each with the same matrix polymer, the simple correlation between leaflet stiffness and Young's modulus cannot be applied. Due to the complexity of the material properties, there is not a simple and obvious relation to their hydrodynamic function.

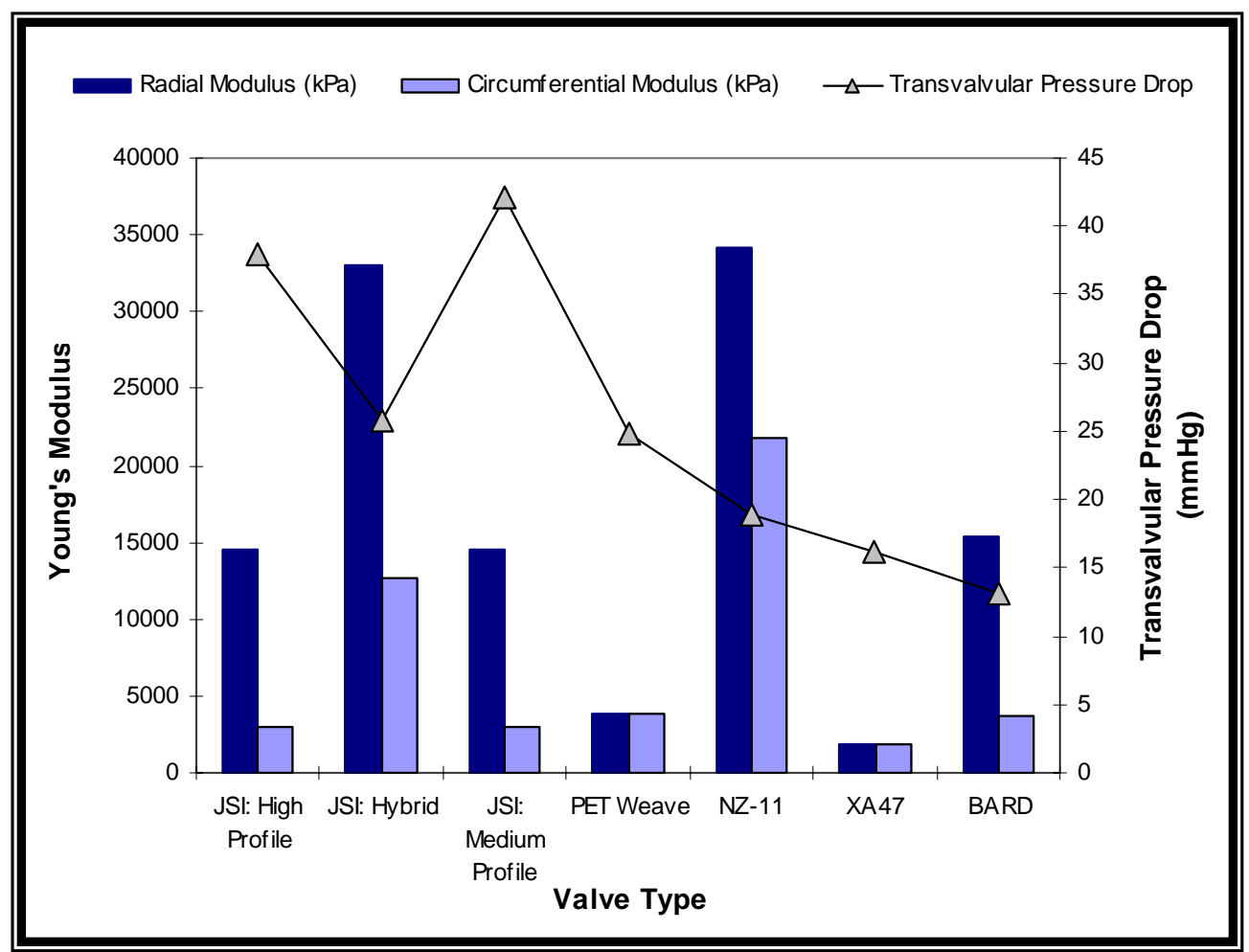

Figure 59. Circumferential and radial Young's modulus versus mean transvalvular pressure drop for all prototype valves

\subsubsection{Valve Fatigue}

The valves whose hydrodynamics were assessed in Section 5.2.2.2 were subsequently placed on the Vivitro HiCycle fatigue tester to evaluate their long-term 
fatigue response. The two most successful valve designs, in terms of their durability, included the BARD-reinforced valve and the JSI-reinforced valve. A comparison of all valves, including those already discussed in Section 5.1, is provided in Table 13, with a short description of their failure modes. The preliminary evaluation from Section 5.1 already established that the optimal design included the cylinder valve design and a $48.5 \%$ styrene, medium profile stent, so the purpose of this evaluation was to determine what leaflet properties are most favorable for the development of a fiber-reinforced polymer trileaflet valve.

Table 13. Summary of the primary valve designs subjected to fatigue evaluation.

\begin{tabular}{|c|c|c|c|c|}
\hline \multicolumn{2}{|r|}{ Valve } & \multirow{2}{*}{$\begin{array}{c}\text { Orientation of } \\
\text { Max } \\
\text { Compliance }\end{array}$} & \multirow{2}{*}{$\begin{array}{c}\begin{array}{c}\text { Years To } \\
\text { Failure }\end{array} \\
0.628\end{array}$} & \multirow{2}{*}{$\begin{array}{l}\text { Failure Mode } \\
\text { - Leaflet/Stent detachment } \\
\text { - Polymer degradation / blowout }\end{array}$} \\
\hline 1 & $\begin{array}{l}\text { LARS 3-Leaflet Valve, } \\
\text { Implant } 1\end{array}$ & & & \\
\hline 2 & $\begin{array}{l}\text { JSI: High Profile, } 30 \% \\
\text { Styrene, Dip, Implant } 2\end{array}$ & Circumferential & 10.35 & $\begin{array}{l}\text { 3-fold increase in pressure drop } \\
\text { - NOT VALVE RELATED }\end{array}$ \\
\hline 3 & $\begin{array}{c}\text { JSI: High Profile, } \mathbf{3 0 \%} \\
\text { Styrene, Dip }\end{array}$ & Radial & $\begin{array}{l}2.72 \\
6.20\end{array}$ & $\begin{array}{l}\text { - Stent fracture } \\
\text { - Hole formation in leaflet belly }\end{array}$ \\
\hline 4 & $\begin{array}{c}\text { JSI: Medium Profile, } \\
\text { 48.5\% Styrene, } \\
\text { Hybrid, Implant } 3\end{array}$ & Circumferential & 1.50 & - Heat seal joint failure \\
\hline 5 & $\begin{array}{l}\text { JSI: Medium Profile, } \\
\text { 48.5\% Styrene, Dip }\end{array}$ & Circumferential & N/A & $\mathrm{N} / \mathrm{A}-4.19 \mathrm{yrs}$ to date \\
\hline 6 & $\begin{array}{l}\text { PET Weave: Medium } \\
\text { Profile, } 48.5 \% \text { Styrene }\end{array}$ & Circumferential & 2.94 & $\begin{array}{l}\text { - Tear formation at all coaptation } \\
\text { regions } \\
\text { - Free edge fraying }\end{array}$ \\
\hline 7 & $\begin{array}{c}\text { NZ-11: Medium } \\
\text { Profile, 48.5\% Styrene }\end{array}$ & Circumferential & 0.20 & - Catastrophic mesh failure \\
\hline 8 & $\begin{array}{l}\text { XA47: Medium Profile, } \\
\text { 48.5\% Styrene }\end{array}$ & Circumferential & $\begin{array}{l}2.34 \\
2.39\end{array}$ & $\begin{array}{l}\text { - Polymer degradation } \\
\text { - Mesh degradation } \\
\text { - Stent fracture }\end{array}$ \\
\hline 9 & $\begin{array}{l}\text { BARD: Med Profile, } \\
\text { 48.5\% Styrene }\end{array}$ & Circumferential & 4.19 & $\begin{array}{l}\text { - Degradation of the heat-sealed } \\
\text { joint }\end{array}$ \\
\hline
\end{tabular}

The failure mode descriptions for valves $1-3$ have already been discussed in detail, so results from valves $4-9$ will be compared. Valves $4-9$ include those cylinder 
valve designs that are mounted on a $48.5 \%$ styrene stent. The NZ-11 PET mesh valve suffered from very early catastrophic failure. After cycling for an equivalent of 0.20 years, a tear initiated and propagated along the leaflet-stent attachment zone of one leaflet (Figure 60). It should be noted that this location was not the location of the heat-sealed joint, as has been the case with other designs. On that same leaflet, a tear developed at the central region of the coaptation zone (Figure 61). This catastrophic breakdown of the reinforcement fabric after such a short period of cycling ruled it out as a potential reinforcement fabric for the polymer valve design. Suturing of the fabric to the stent was assumed to contribute to the premature failure of the valve. During the manufacturing process, it was noted that hole formation and mesh separation developed at the suture locations; nevertheless, the tear at the center of the coaptation could not be attributed to suturing, so even if a new leaflet-stent attachment technique were developed, the reinforcement would still be considered inappropriate for use.

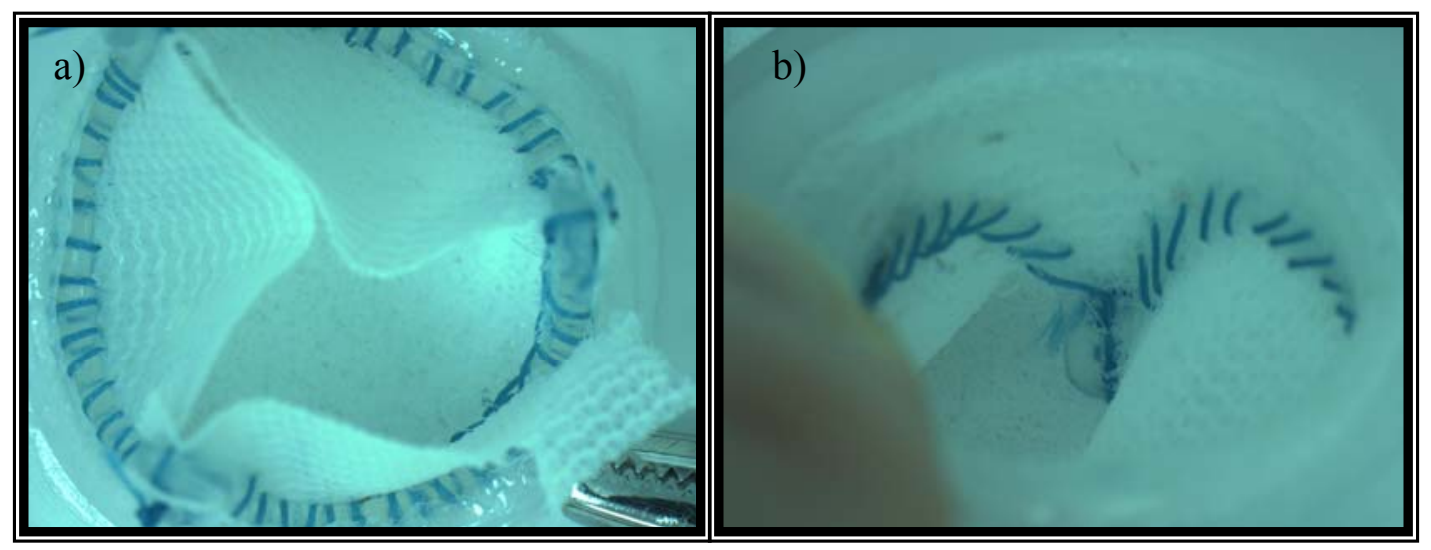

Figure 60. NZ-11 valve showing tear formation along the leaflet-stent zone after an equivalent of 0.20 years of cycling. Tear viewed from the a) aortic and b) ventricular orientation 


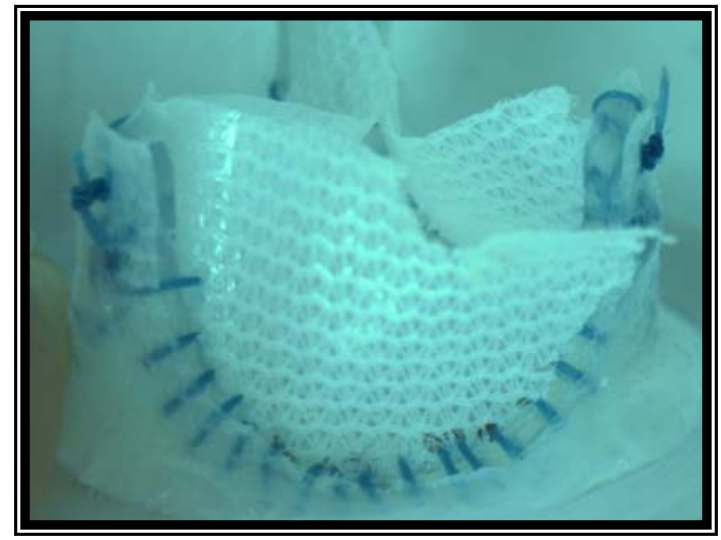

Figure 61. NZ-11 valve showing tear formation at the center of coaptation of the same leaflet as above.

Two XA-47 PET mesh valves were manufactured for testing of valve fatigue. The first valve lasted an equivalent of 2.34 years before one of the supporting stent posts fractured completely (Figure 62a). In addition, tear formation at the center of coaptation of two of the leaflets (Figure 62b), and polymer blowout at the center of coaptation for the third leaflet was evident (Figure 62c). The second of the XA-47 valves failed at an equivalent of 2.39 years of cycling. It had very similar leaflet failure characteristics, with the addition of degradation of the heat-sealed joint. The stent on this valve did, however, maintain its integrity. Both valves appeared to have problems related to delamination between the leaflet polymer and reinforcement (Figure 62d). While using the solventcasting technique to manufacture these samples, problems arose with bubble formation and reinforcement wetting. The fabric appeared to be resistant to wetting by the SIBS/toluene solution. This was assumed to result in mainly surface coating by the polymer, leaving entrapped air within the mesh and contributing to the delamination. Ignoring the delaminations seen, the underlying mesh itself did not have the structural integrity to maintain long-term cycling, so this reinforcement type was also determined to be insufficient for use in a trileaflet valve. 


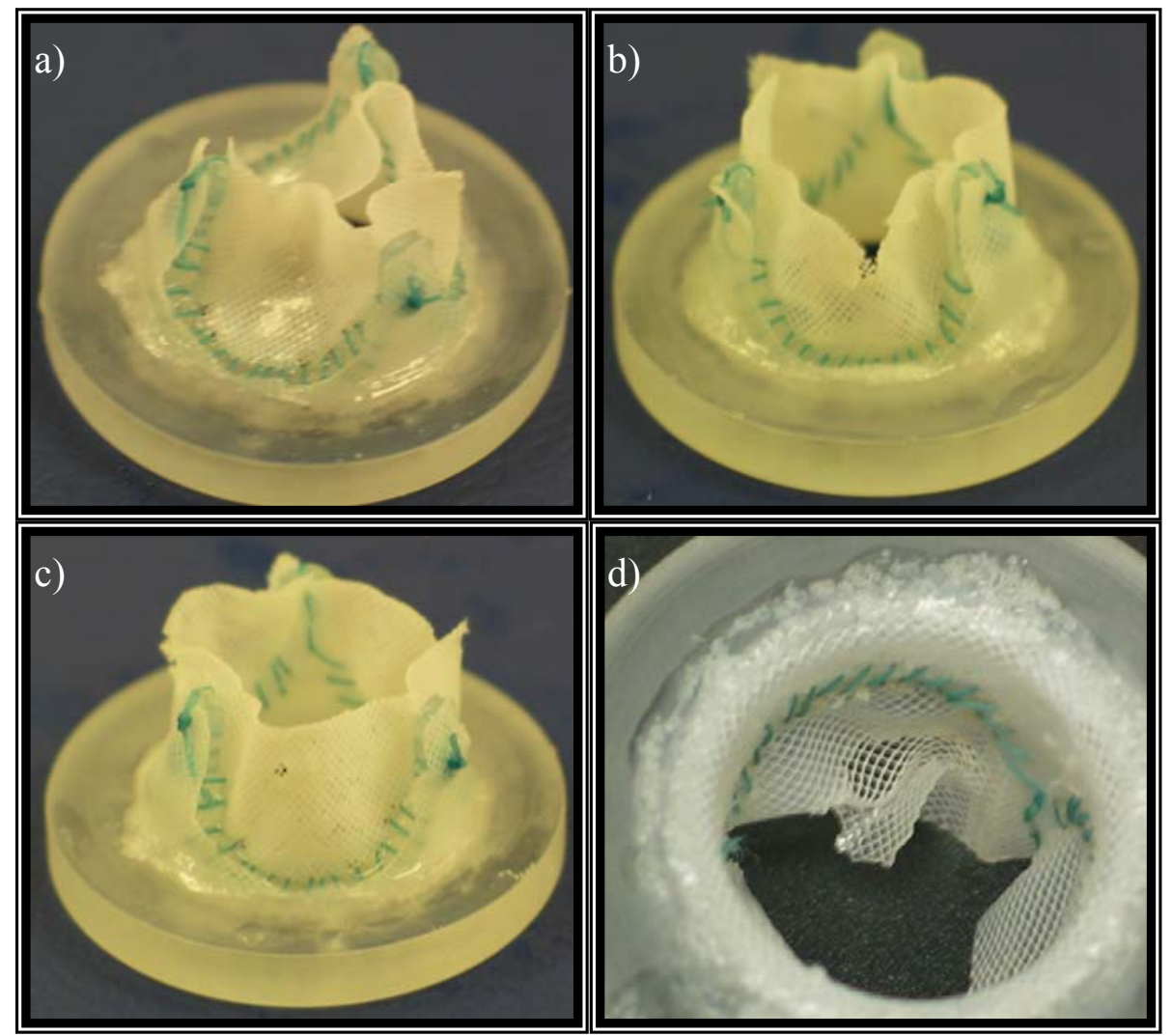

Figure 62. XA-47 valve showing a) stent fracture, b) tear formation at the coaptation, c) polymer blowout and d) delamination at the coaptation.

One of the downfalls of the PET weave reinforced valve is that the weave is a non-locking weave, so fibers are free to move around. The weave valve discussed in the preliminary testing incorporated the same weave that has been tested here, but a different orientation was applied. In the previous study, the weave was oriented so that the fibers were oriented along the radial and circumferential directions. This led to an uncharacteristically high modulus in these two directions, and it promoted free edge fraying by not securing the fibers located right at the free edge. By rotating the orientation $45^{\circ}$, greater radial and circumferential compliance could be achieved, and the fibers could be held more securely within the polymer matrix. An increase in valve durability was seen (from 0.943 years to 2.94 years), but the reinforcement fabric was 
again determined to be inappropriate. Valve failure for the PET weave valve that allowed circumferential and radial compliance was as a result of tear formation of the leaflet along two of the three commissures (Figure 63). The actual weave fibers fractured, causing valve prolapse. Even with this new fiber orientation, fraying at the free edge of the leaflet was still evident (Figure 63b).

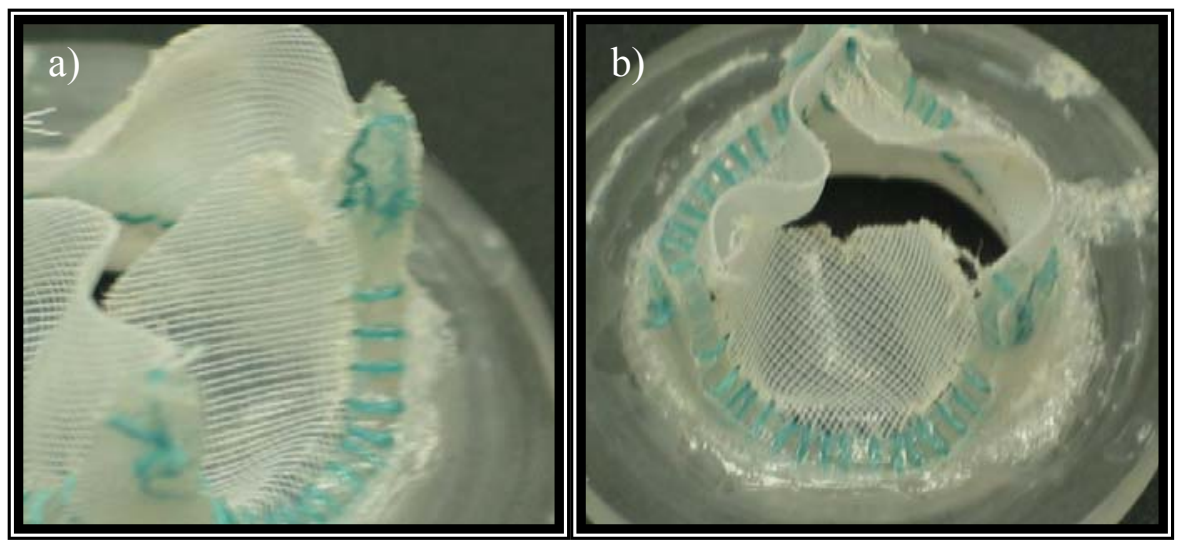

Figure 63. PET Weave valve showing a) tear formation at the commissure and b) leaflet prolapse and free edge fraying.

The two remaining valves were both PET knit reinforced valves that differed with respect to leaflet thickness. The JSI valve had a leaflet thickness of $0.40 \mathrm{~mm}$, while the BARD valve had a $0.20 \mathrm{~mm}$ thickness. The tensile tests of these materials and subsequent statistical analyses (Section 5.2.2.1) revealed that the Young's moduli in both the circumferential and radial directions were not significantly different. Upon in vitro fatigue analysis of the two valves, failure of the BARD-reinforced valve occurred at an equivalent of 4.19 years as a result of heat seal deterioration. The actual reinforcement fabric, and even the valve as a whole, was still in good working condition, but the fabrication method used to seal the flat solvent cast BARD sheet into a cylinder proved to 
once again be insufficient. The JSI-reinforced valve showed no deterioration at this stage of cycling; however, the Vivitro HiCycle has to always be balanced, so it needs a minimum of 2 valves to be run simultaneously. Due to the lack of a replacement valve after the BARD valve failed, the cyclic fatigue of the JSI valve had to be halted. The JSI valve had also been cycled an equivalent of 4.19 years at this stage. Both the JSI and BARD valves were subjected to a hydrodynamic evaluation after 66 million cycles (1.65 years). Less than a $1 \mathrm{mmHg}$ change in transvalvular pressure drop and a $2 \%$ changed in percent regurgitation was seen for both valves when results were compared with their baseline, pre-fatigue data. It was concluded that fatigue to this point had not impacted the acute function of the valves. The failure mode experienced by the BARD valve was not sufficient to eliminate it as a potential leaflet reinforcement material. In fact, it is the only viable reinforcement material out of all of the versions that have been tested. The dip-coated JSI reinforcement had previously been eliminated due to unfavorable in vivo response.

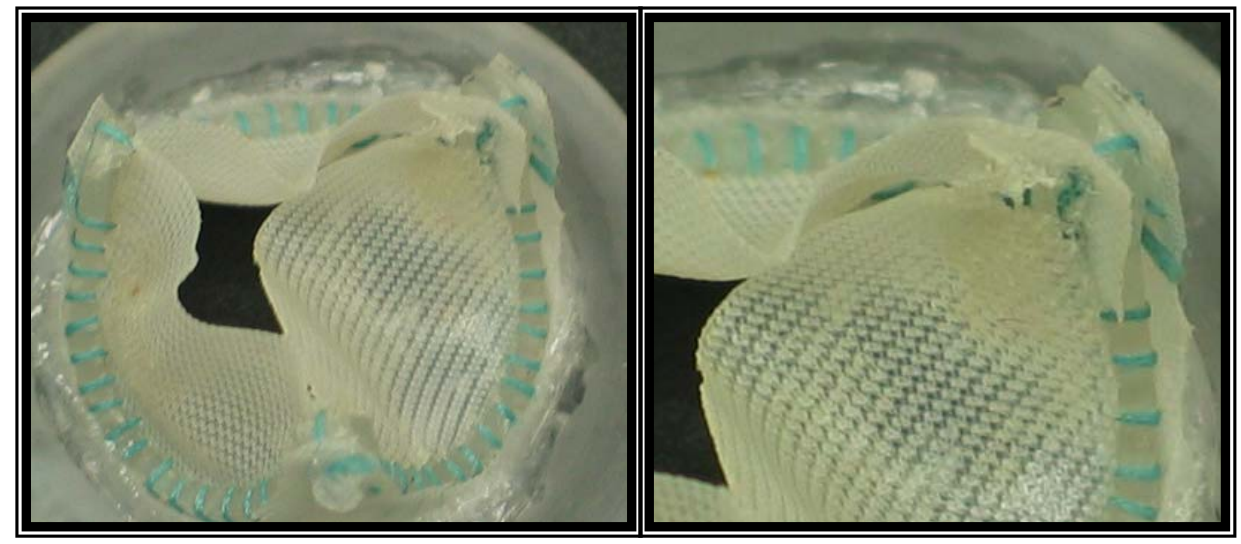

Figure 64. BARD valve showing deterioration of the heat-sealed joint. 
It has been proposed by Bernacca et al. [64] that a high modulus material could be more resistant to fatigue. A plot of the circumferential and radial moduli versus fatigue life (Figure 65) shows no distinct pattern exists for the fiber-reinforced SIBS valves. Once again, the complex interaction within each orthotropic material invalidates such a simplistic interaction between fatigue life and leaflet modulus. Interactions within the material exist, and a finite element analysis could provide a better predictor of fatigue life based on an analysis of the stress concentrations within a leaflet subjected to a physiological load.

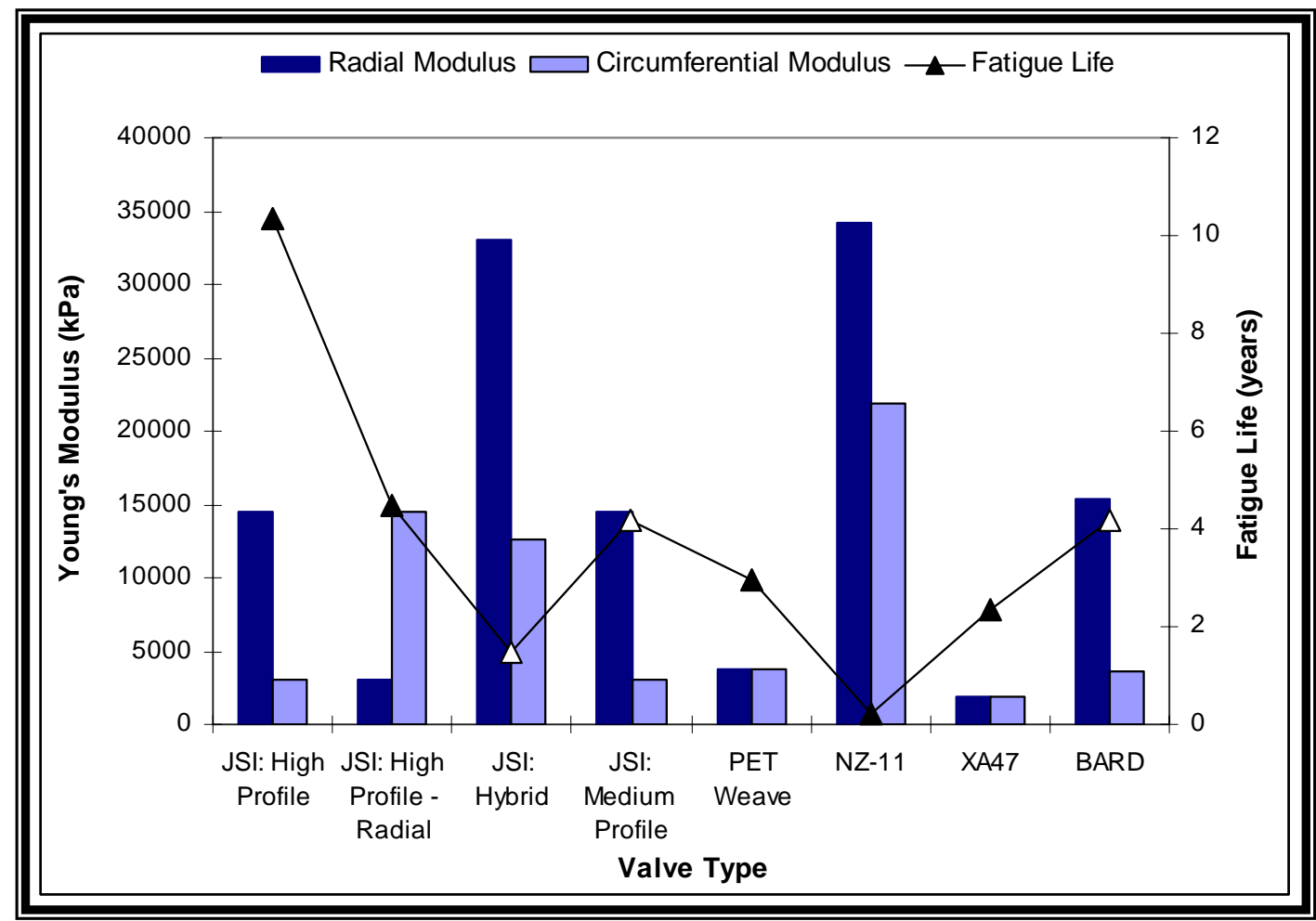

Figure 65. Plot of fatigue life versus the radial and circumferential moduli.

No SIBS trileaflet valve has thus far made it to an equivalent of 15 years of fatigue. The BARD-reinforced valve appears to be the most promising, but a design 
modification for the leaflet cylinder sealing technique is required for this to be a possibility.

\subsubsection{Finite Element Model}

Eight separate combinations of valve geometry and material properties were compared to assess the impact of 1) stent flexibility and geometry, 2) leaflet anisotropy, and 3) leaflet coaptation geometry on the stress concentrations in the leaflet as a result of pressure loading. One particular valve, the BARD-reinforced valve mounted on a medium profile, $48.5 \%$ styrene stent, was manufactured and its stent deflection and transient geometry were quantified for comparison and validation of the finite element model. Each valve was subjected to a quasi-static diastolic loading regimen to assess the resultant stress concentrations when the valve was subjected to its maximum pressure load.

For the quasi-static analysis, it was the initial intention to subject the valve to the incremental loading steps representing the entire cardiac cycle, i.e. diastolic and systolic loading. What was found was that the static, implicit solution technique was not equipped to deal with the numerical instabilities that occurred when a complete inversion of curvature or buckling occurred, such as what happens during systole. For all quasistatic models, the analysis terminated during systole. As per a discussion with Michel Labrosse (University of Ottawa, Department of Mechanical Engineering, Ottawa, Ontario, Canada), it was recommended that the dynamic explicit solution procedure in ABAQUS was more suitable for analysis of heart valve leaflets subject to buckling, so analysis of the full cardiac loading cycle is proposed for future analyses with this method. 


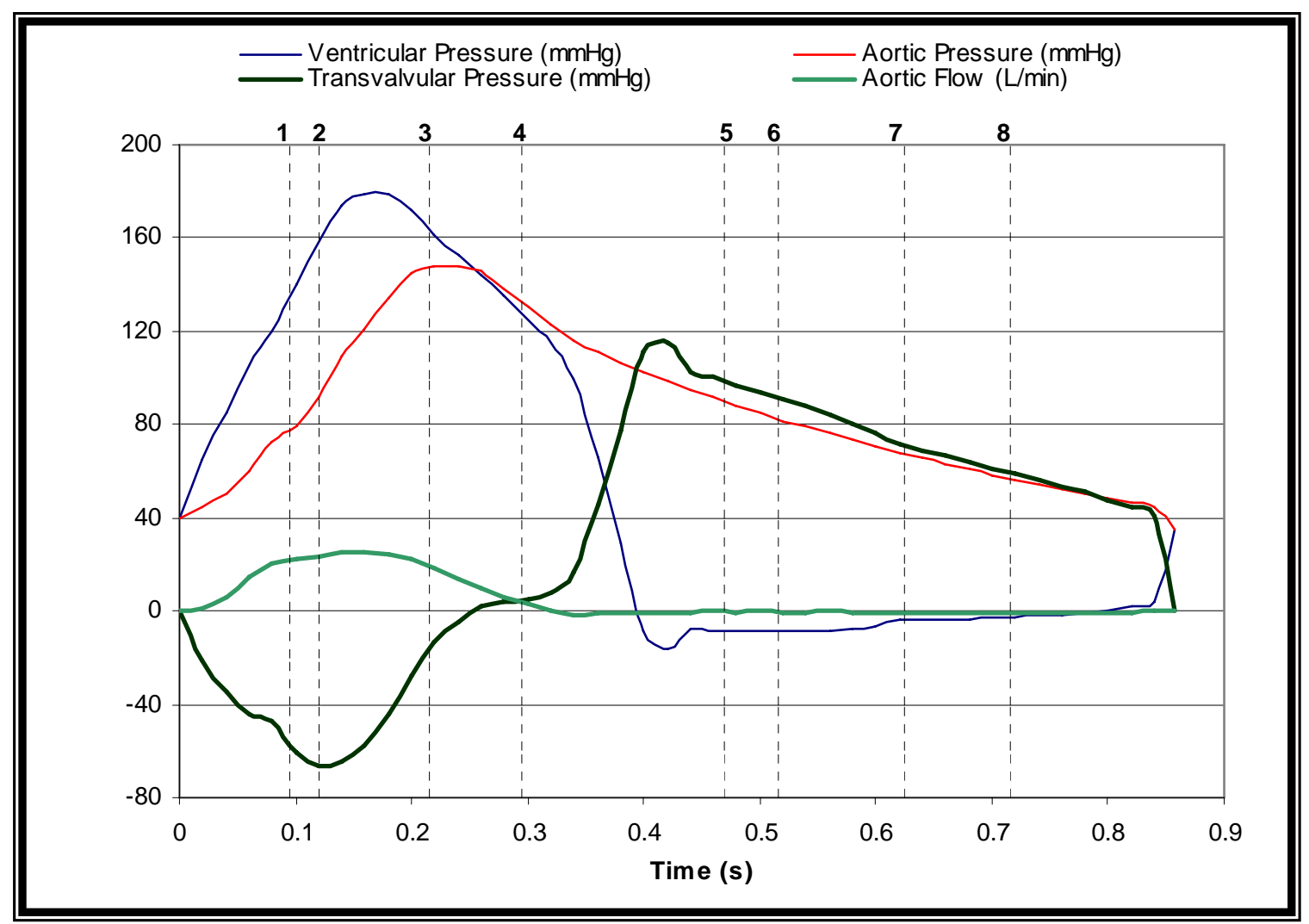

Figure 66. Hydrodynamic data showing the flow and pressure waveforms. The dashed lines $(1-8)$ represent the specific locations during the cardiac cycle where each image pair was triggered.

\subsubsection{1. $\quad$ Model Validation}

\subsection{Transient Geometry Analysis}

During the DCSP procedure, the eight images that were taken during the cardiac cycle corresponded to points 1 through 8 on Figure 66. An additional image was taken with the hydrodynamic tester switched off, thereby allowing imaging of the valve under a $0 \mathrm{mmHg}$ transvalvular pressure load. The 3-D coordinates of the fiducial points were derived in Photomodeler ${ }^{\circledR}$, and the generated scatterplots in addition to the raw image pairs are shown in Figure 67 - Figure 72 and in Appendix IV: Figure 107 - Figure 118. 


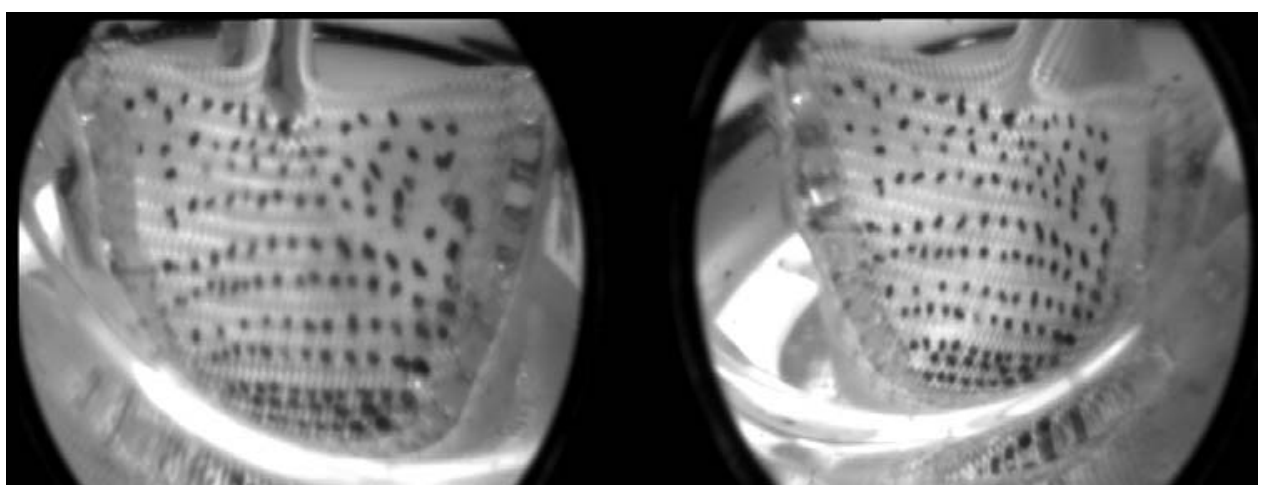

Figure 67. Photogrammetry image pairs corresponding to the baseline image where the valve was under a no load condition.

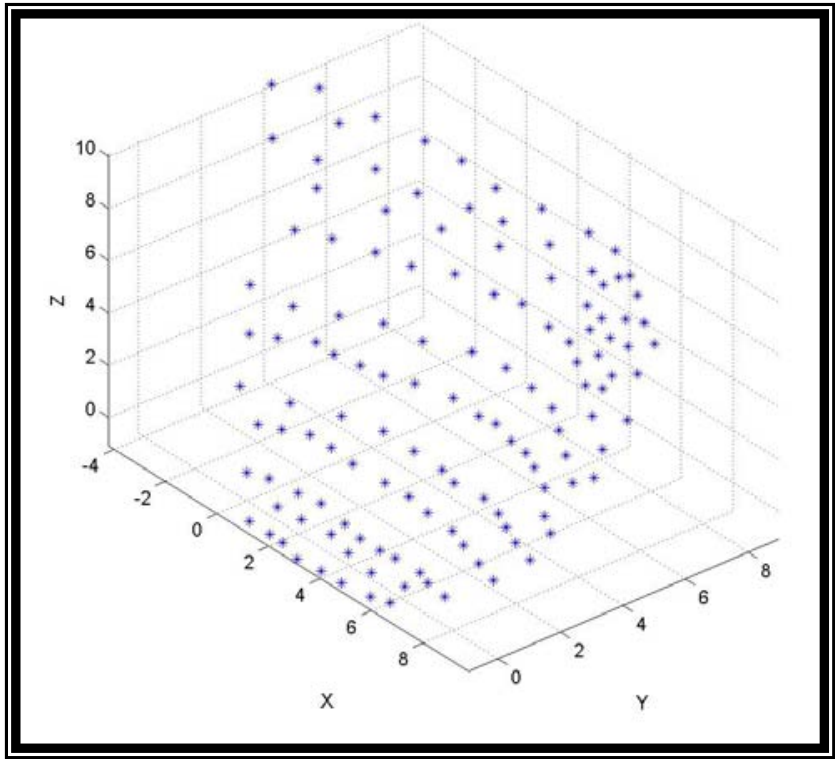

Figure 68. MatLab regeneration of fiducial points from the $\mathrm{x}, \mathrm{y}$, and $\mathrm{z}$ coordinated generated in Photomodeler® from Figure 67.

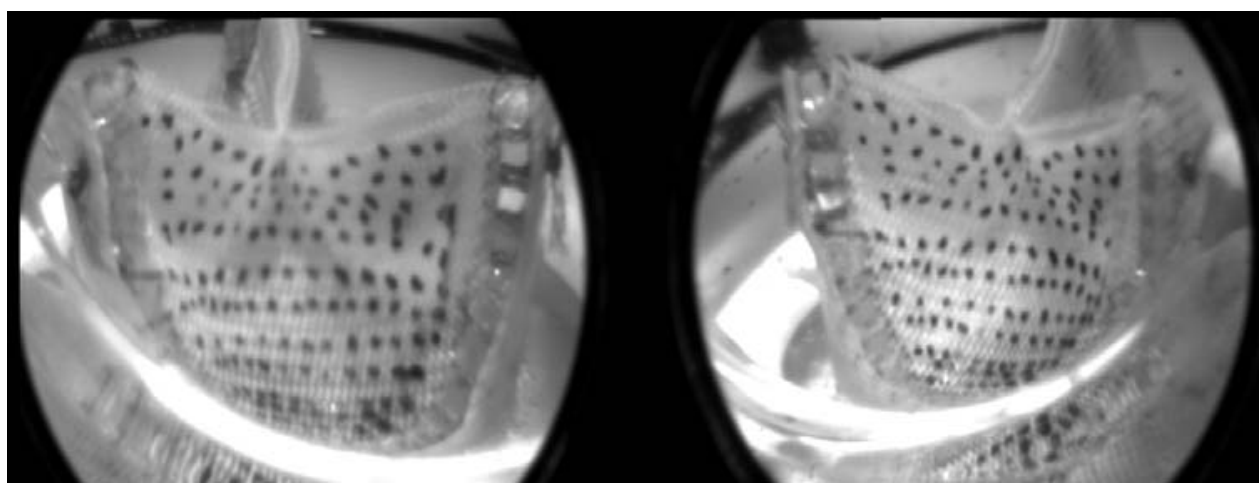

Figure 69. Photogrammetry image pairs corresponding to Frame 5 in Figure 66. 


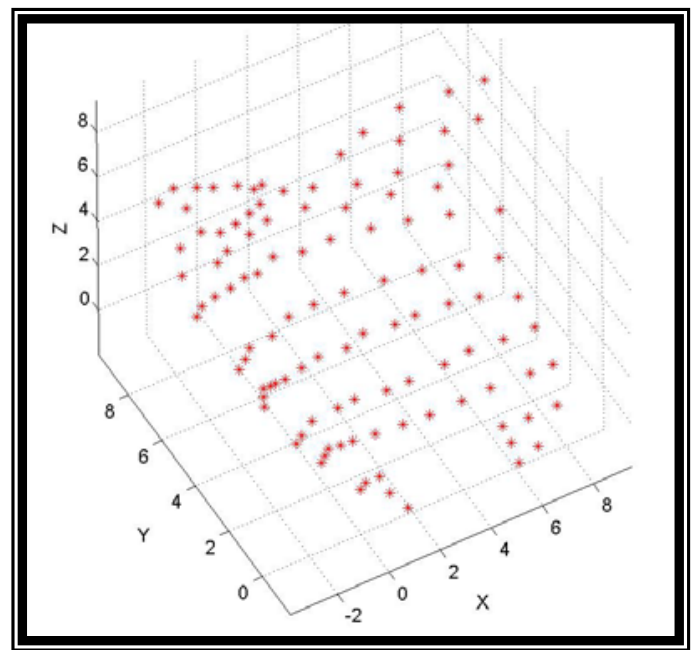

Figure 70. MatLab regeneration of fiducial points from the $\mathrm{x}, \mathrm{y}$, and $\mathrm{z}$ coordinated generated in Photomodeler ${ }^{\circledR}$ from Figure 69.

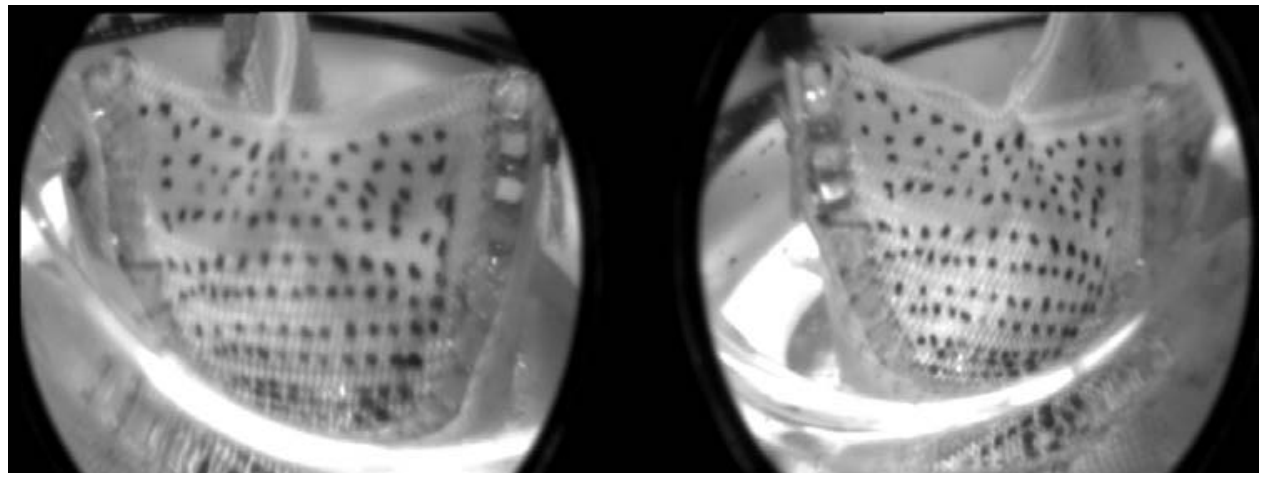

Figure 71. Photogrammetry image pairs corresponding to Frame 7 in Figure 66.

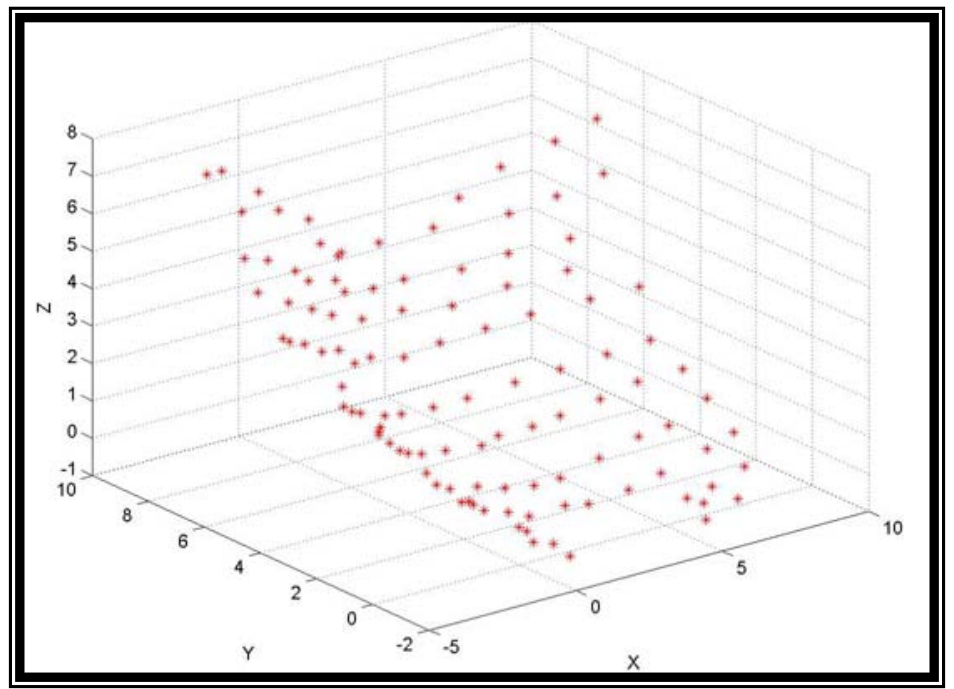

Figure 72. MatLab regeneration of fiducial points from the $\mathrm{x}, \mathrm{y}$, and $\mathrm{z}$ coordinated generated in Photomodeler® from Figure 71. 
Table 14. Validation of the quasi-static FEM. The particular valve included the BARD-reinforced leaflets with slight coaptation curvature. These leaflets were mounted on the medium profile, $48.5 \%$ styrene stent.

\begin{tabular}{|c|c|c|c|c|c|c|}
\hline Frame & $\begin{array}{c}\text { Equivalent } \\
\text { ABAQUS } \\
\text { Time (s) }\end{array}$ & $\begin{array}{c}\text { Actual } \\
\text { ABAQUS } \\
\text { Time (s) }\end{array}$ & Points & $\begin{array}{c}\text { DCSP } \\
\text { Measure } \\
(\mathrm{mm})\end{array}$ & $\begin{array}{c}\text { ABAQUS } \\
\text { Measure } \\
(\mathbf{m m})\end{array}$ & $\begin{array}{l}\text { Percentage } \\
\text { Error }\end{array}$ \\
\hline \multirow{3}{*}{ Baseline } & \multirow{3}{*}{0.0} & \multirow{3}{*}{0.0} & $1-2$ & 6.39 & 5.60 & 14.12 \\
\hline & & & $1-3$ & 9.15 & 7.81 & 17.05 \\
\hline & & & $2-3$ & 5.01 & 4.61 & 8.68 \\
\hline \multirow{3}{*}{7} & \multirow{3}{*}{0.5} & \multirow{3}{*}{0.4802} & $1-2$ & 7.16 & 5.65 & 26.76 \\
\hline & & & $1-3$ & 9.11 & 8.00 & 13.86 \\
\hline & & & $2-3$ & 5.29 & 4.63 & 14.17 \\
\hline \multirow{3}{*}{5} & \multirow{3}{*}{0.6} & \multirow{3}{*}{0.5714} & $1-2$ & 4.22 & 9.96 & 57.57 \\
\hline & & & $1-3$ & 12.01 & 13.27 & 9.49 \\
\hline & & & $2-3$ & 10.19 & 4.63 & 120.13 \\
\hline
\end{tabular}

As is apparent in Figure 66, no images were taken during the diastolic "loading" of the valve (between images 4 and 5); images were only available for the diastolic "unloading". As a result, the quasi-static model had to be evaluated using the image pairs taken during the diastolic "unloading", with the assumption that the leaflet will maintain the same geometry at a given pressure load, regardless as to whether the pressure is increasing or decreasing. The image pairs from frames 5 (Figure 69 and Figure 70) and 7 (Figure 71 and Figure 72) were used and the equivalent time for the image sequence was calculated for comparison with the finite element model. The distances between each of the three points selected for quantitative valve validation were computed, and the results from the DCSP and finite element model are compared below (Table 14).

The percentage error obtained between the two measurements was very large, ranging from $8.68 \%$ to $120.13 \%$. Even when no load was applied on the valve (i.e. the baseline measure), the percentage error between experimental and model data was high. This high error was not sufficient to rule out the model as being a feasible representation of the real case for the following reasons: 1) The model represents the optimal case where the valve is completely symmetrical and each of the three leaflet's opening and closing 
geometries are identical. In the real case, manufacturing restrictions prevent this perfect leaflet symmetry, so leaflets do not behave exactly the same. 2) The set-up for the DCSP was not the most favorable as it was impossible to calibrate the cameras in the exact same environment as that in which the images were taken. Calibration of the cameras requires a minimum of eight separate images from eight separate angles, including from above the image plane, and the Vivitro tester restricts the views from all of the eight angles. As a result, the cameras had to be calibrated in the blood analog fluid without the presence of the acrylic walls and glass sinus of the Vivitro. These objects are capable of distorting the image and providing a less than optimal image regeneration. 3) Due to the high rate of diastolic leaflet loading, it was difficult to capture an image during this phase, and images during diastolic "unloading" had to be substituted. The combination of all three of these factors are believed to have contributed to the high percentage error between the experimental and model data, and it was concluded that this method neither proves nor disproves the validity of the model. It is believed that this method is feasible for the validation of the model if a more ideal experimental set-up is instituted. The poor correlation between these results prompted the second validation, where stent deflection was quantified and compared with the model.

\subsubsection{2. $\quad$ Stent Deflection}

Stent deflection of the same experimental valve examined by DCSP was quantified during the hydrodynamic evaluation at $70 \mathrm{BPM}$ and $5.6 \mathrm{~L} / \mathrm{min}$. The valve was imaged from above, and the maximum deformation at peak diastole and peak systole was quantified by measuring the post-to-post distances. For the quasi-static model, the maximum deflection at peak diastole was compared with that for the finite element 
model. The finite element model predicted an average post-to post distance of $12.21 \mathrm{~mm}$, while the experimental set-up predicted an average deflection of $13.26 \mathrm{~mm}$. This resulted in an $8.58 \%$ error, which was determined to be sufficient to prove the validity of the finite element model.

\subsubsection{2. $\quad$ Material Properties}

The material properties needed for input into the quasi-static models are provided in Table 15 and Table 16. The through thickness modulus $\left(\mathrm{E}_{3}\right)$ could not me measured, so it was assumed to be that of the isotropic polymer. According to Skinner [66] this is a valid assumption because, when the applied stress is perpendicular to the reinforcement fibers, the modulus of elasticity mimics that of the surrounding matrix.

Table 15. Orthotropic material engineering constants used as input into the quasi-static FEM. Note all Poisson's ratio values were assumed to be zero.

\begin{tabular}{|c|c|c|c|c|c|c|c|c|c|}
\hline & $\begin{array}{c}\mathbf{E}_{1} \\
\left(\mathbf{N} / \mathbf{m m}^{2}\right)\end{array}$ & $\begin{array}{c}\mathbf{E}_{2} \\
\left(\mathbf{N} / \mathbf{m m}^{2}\right)\end{array}$ & $\begin{array}{c}\mathbf{E}_{3} \\
\left(\mathbf{N} / \mathbf{m m}^{2}\right)\end{array}$ & $\mathbf{v}_{12}$ & $\mathbf{v}_{13}$ & $\mathbf{v}_{23}$ & $\begin{array}{c}\mathbf{G}_{12} \\
\left(\mathbf{N} / \mathbf{m m}^{2}\right)\end{array}$ & $\begin{array}{c}\mathbf{G}_{13} \\
\left(\mathbf{N} / \mathbf{m m}^{2}\right)\end{array}$ & $\begin{array}{c}\mathbf{G}_{23} \\
\left(\mathbf{N} / \mathbf{m m}^{2}\right)\end{array}$ \\
\hline $\begin{array}{c}\text { JSI - } \\
\text { Circumferential }\end{array}$ & 2.972 & 14.504 & 1.064 & 0 & 0 & 0 & 1.486 & 1.486 & 7.252 \\
\hline JSI - Radial & 14.504 & 2.973 & 1.064 & 0 & 0 & 0 & 7.252 & 7.252 & 1.486 \\
\hline $\begin{array}{c}\text { BARD - } \\
\text { Circumferential }\end{array}$ & 3.670 & 15.351 & 1.064 & 0 & 0 & 0 & 1.835 & 1.835 & 7.675 \\
\hline
\end{tabular}

Table 16. Isotropic material constants used as input into both the quasi-static and dynamic FEMs.

\begin{tabular}{|c|c|c|}
\hline & $\begin{array}{c}E \\
\left(\mathbf{N} / \mathbf{m m}^{2}\right)\end{array}$ & v \\
\hline $\mathbf{8 . 5 \%}$ SIBS & 1.064 & 0.4 \\
\hline $\mathbf{3 0 \%}$ SIBS & 62.429 & 0.4 \\
\hline $\mathbf{4 8 . 5 \%}$ SIBS & 385.372 & 0.4 \\
\hline
\end{tabular}

Please note that for the orthotropic leaflet materials, the Poisson's ratio had to be assumed to be zero in all cases. This was found to be necessary when running these models because, when a non-zero number was instituted, the simulation was unable to 
converge. By assuming the Poisson's ratios to be zero, a comparison between all cases was achieved, and the impact of changing Young's modulus and leaflet thickness could be assessed. For the isotropic materials, a non-zero Poisson's ratio did not present a problem, so the measured value was input in these cases.

\subsubsection{3. $\quad$ Quasi-Static Model}

For the quasi-static model, the transvalvular pressure load was ramped over time according to the curve shown in Figure 73. The resultant stress concentrations at the maximum load for all eight models are shown in Figure 74 - Figure 81. For the leaflets, images of the stress concentration on both the aortic and ventricular surfaces are provided for comparison. For all models, a high stress point occurred on the inner surface of the stent where the stent post joined the stent base, which is consistent with a failure mode seen in vitro. During dynamic fatigue of the XA-47 reinforced valve and the high-profile JSI-reinforced valve with radial compliance, the stent post failed in this region.

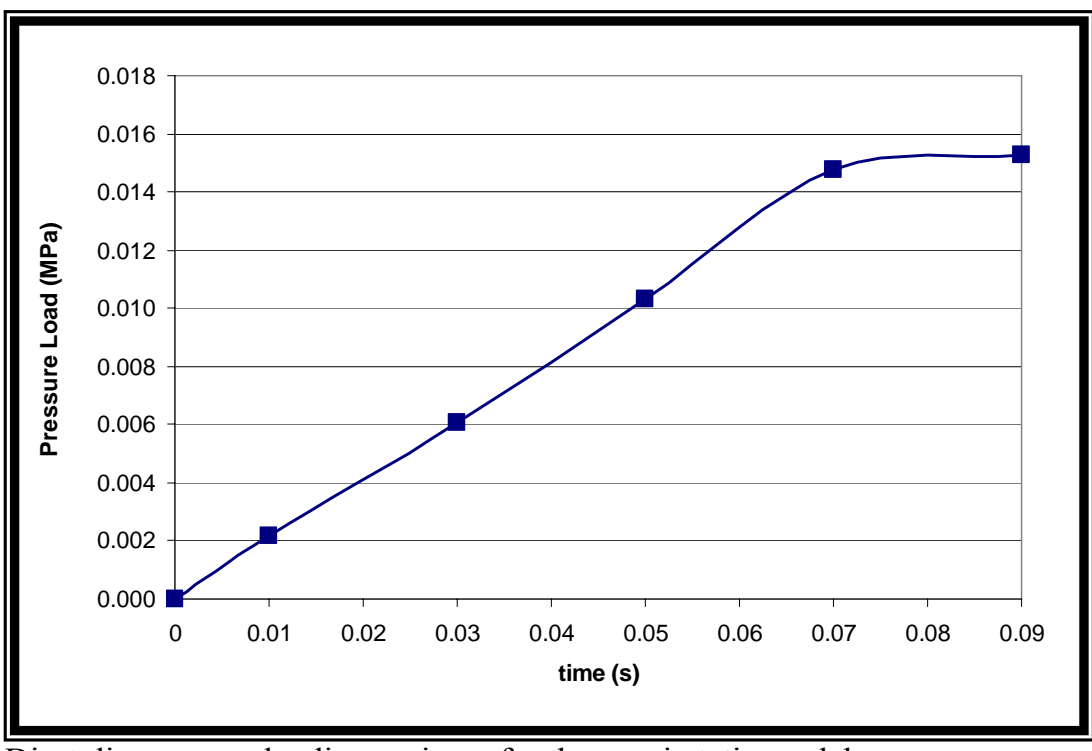

Figure 73. Diastolic pressure loading regimen for the quasi-static model 
When evaluating the top views of all models (Figure 74 - Figure 81), twisting in the coaptation area was noted for all valves except that with the rigid stent. The twisting is symmetrical from one leaflet to the next, and it is believed to be as a consequence of the contact definition. Contact is defined as a pure master-slave interaction, where the slave cannot cross the master surface. Based on how each master-slave pair was defined, the master surface is always the surface that pushes the slave surface and causes the twisting at the valve center. For an actual valve, this twisting does not necessarily occur, as one leaflet may be pushed by both of the adjacent leaflets, forcing these leaflets past the $120^{\circ}$ coaptation zone, such as in the case shown in Figure 82a. Nevertheless, this model interaction was believed to be satisfactory as a similar deformation was shown during hydrodynamic testing of a bioprosthetic valve by Labrosse et al. [65] (Figure 82b). The inward flexion of the posts is believed to contribute to the twisting phenomenon, as it does not occur in the rigid stent case (Figure 75).

The non-reinforced SIBS valve leaflets (Figure 78) were modeled with the same $0.20 \mathrm{~mm}$ thickness as that of the BARD-reinforced leaflets. The SIBS leaflets experienced significantly more deformation than any of the reinforced valve leaflets, proving the insufficiency of the polymer alone. Given the lower Young's modulus, it makes sense that more deformation is seen when the valve is subject to the same loading conditions. From this, it can be concluded that fiber reinforcement is necessary for the normal functioning of the SIBS trileaflet valve when the $8.5 \%$ styrene SIBS is used in the leaflet. Preliminary studies of the SIBS valve comparing isotropic leaflets and reinforced leaflets of the same thickness showed severe leaflet insufficiency due to prolapse, further verifying the need for leaflet reinforcement [9]. 

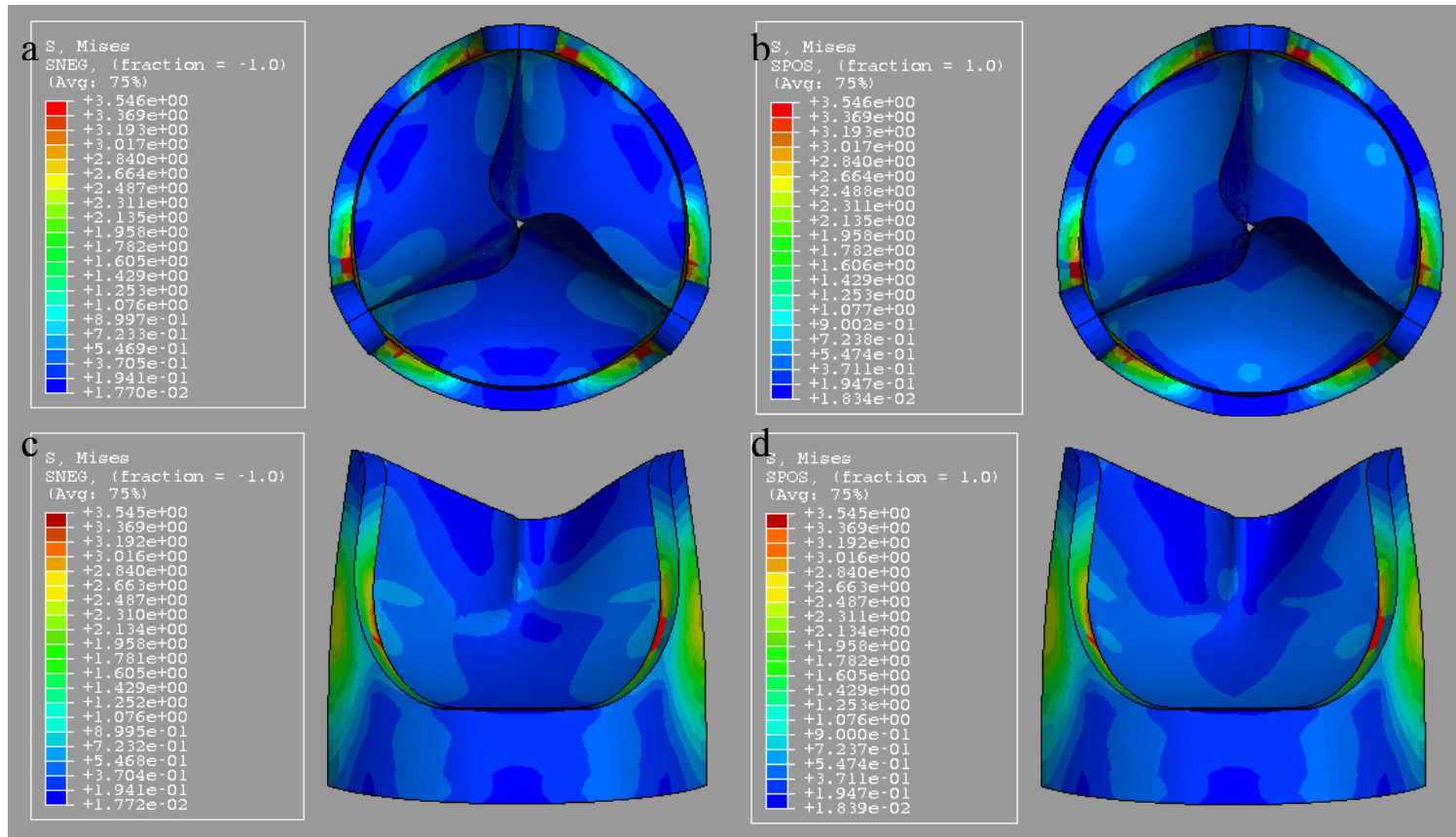

Figure 74. BARD-reinforced leaflets with slight coaptation curvature mounted on a $48.5 \%$ styrene, medium profile stent (BARD-Reg). Views include (a) top view of aortic surface stresses, (b) top view of ventricular surface stresses, (c) front view of one third of the valve showing aortic surface stresses, and (d) front view of one third of the valve showing the ventricular surface stresses.
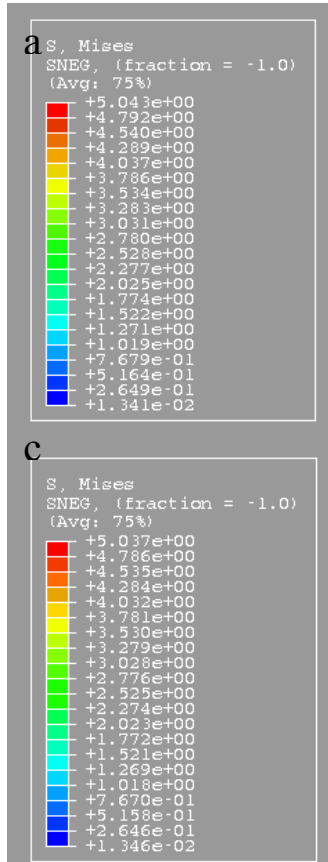
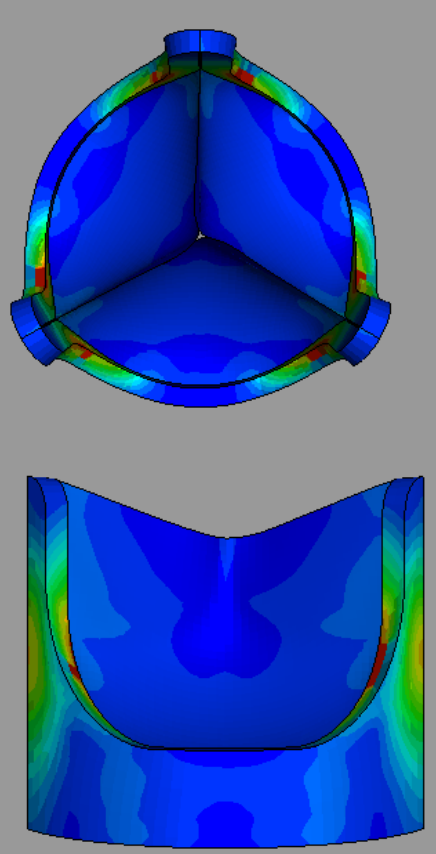
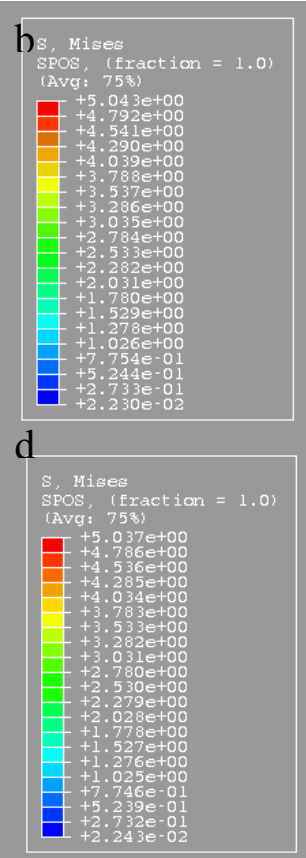
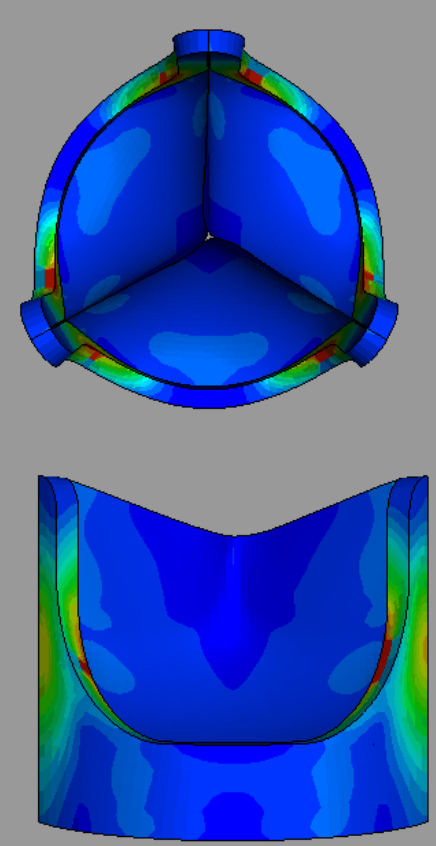

Figure 75. BARD-reinforced leaflets with slight coaptation curvature mounted on a rigid, medium profile stent (BARD-Reg-Rigid). Views include (a) top view of aortic surface stresses, (b) top view of ventricular surface stresses, (c) front view of one third of the valve showing aortic surface stresses, and (d) front view of one third of the valve showing the ventricular surface stresses. 


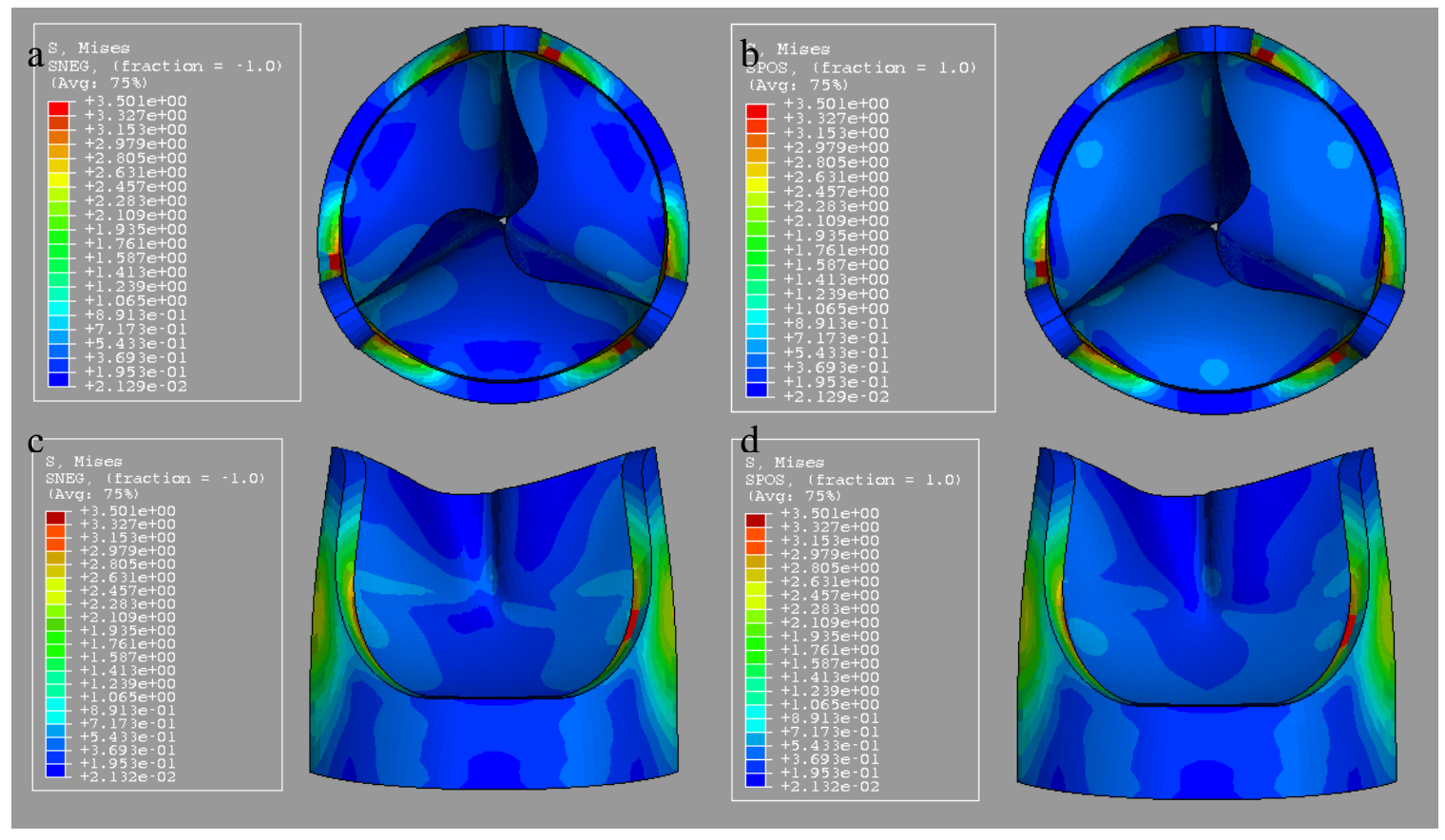

Figure 76. BARD-reinforced leaflet with flat coaptation curvature mounted on a $48.5 \%$ styrene, medium profile stent (BARD-Flat). Views include (a) top view of aortic surface stresses, (b) top view of ventricular surface stresses, (c) front view of one third of the valve showing aortic surface stresses, and (d) front view of one third of the valve showing the ventricular surface stresses.

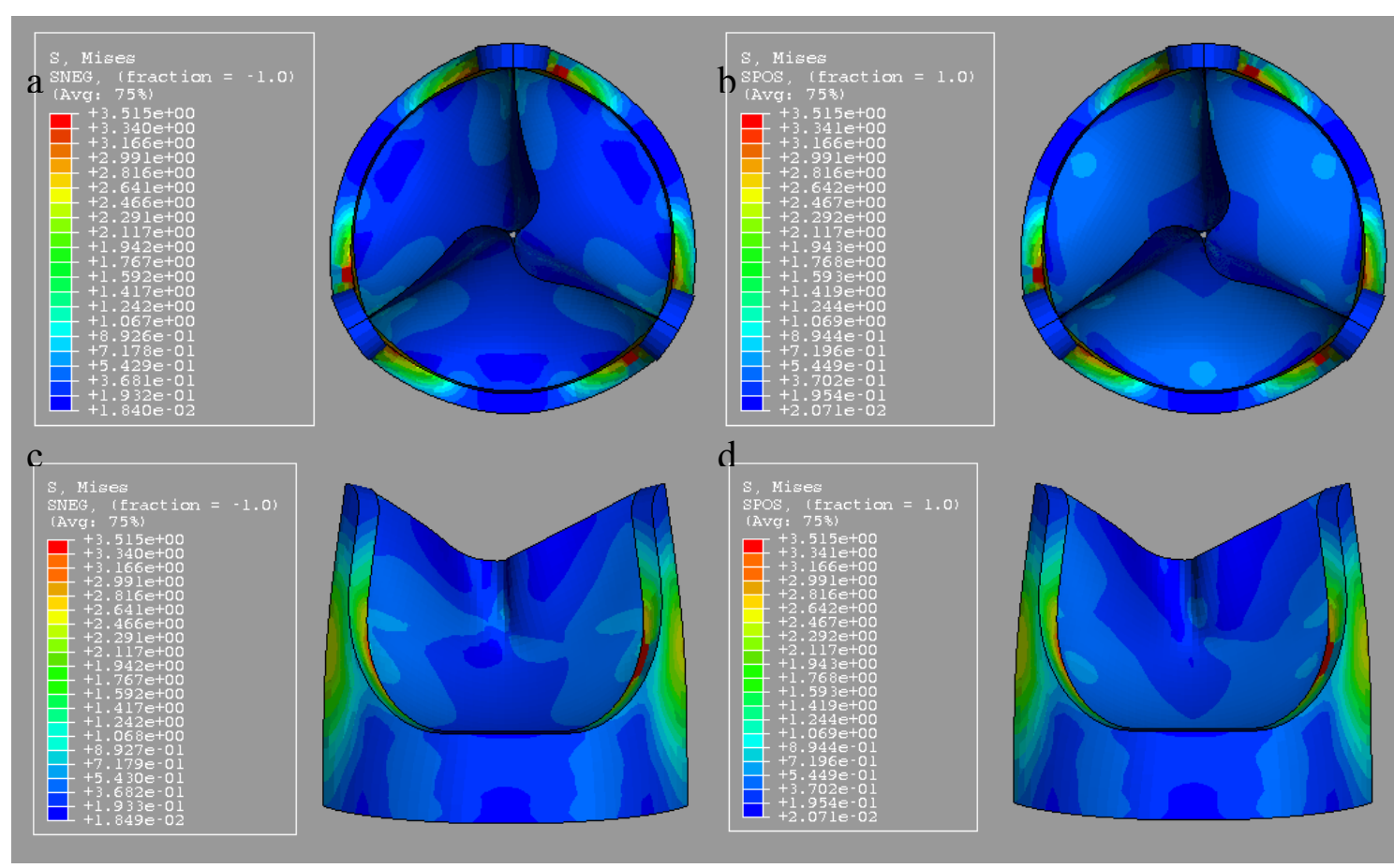

Figure 77. BARD- reinforced leaflet with large coaptation curvature, mounted on a $48.5 \%$ styrene, medium profile stent (BARD-Lrg). Views include (a) top view of aortic surface stresses, (b) top view of ventricular surface stresses, (c) front view of one third of the valve showing aortic surface stresses, and (d) front view of one third of the valve showing the ventricular surface stresses. 


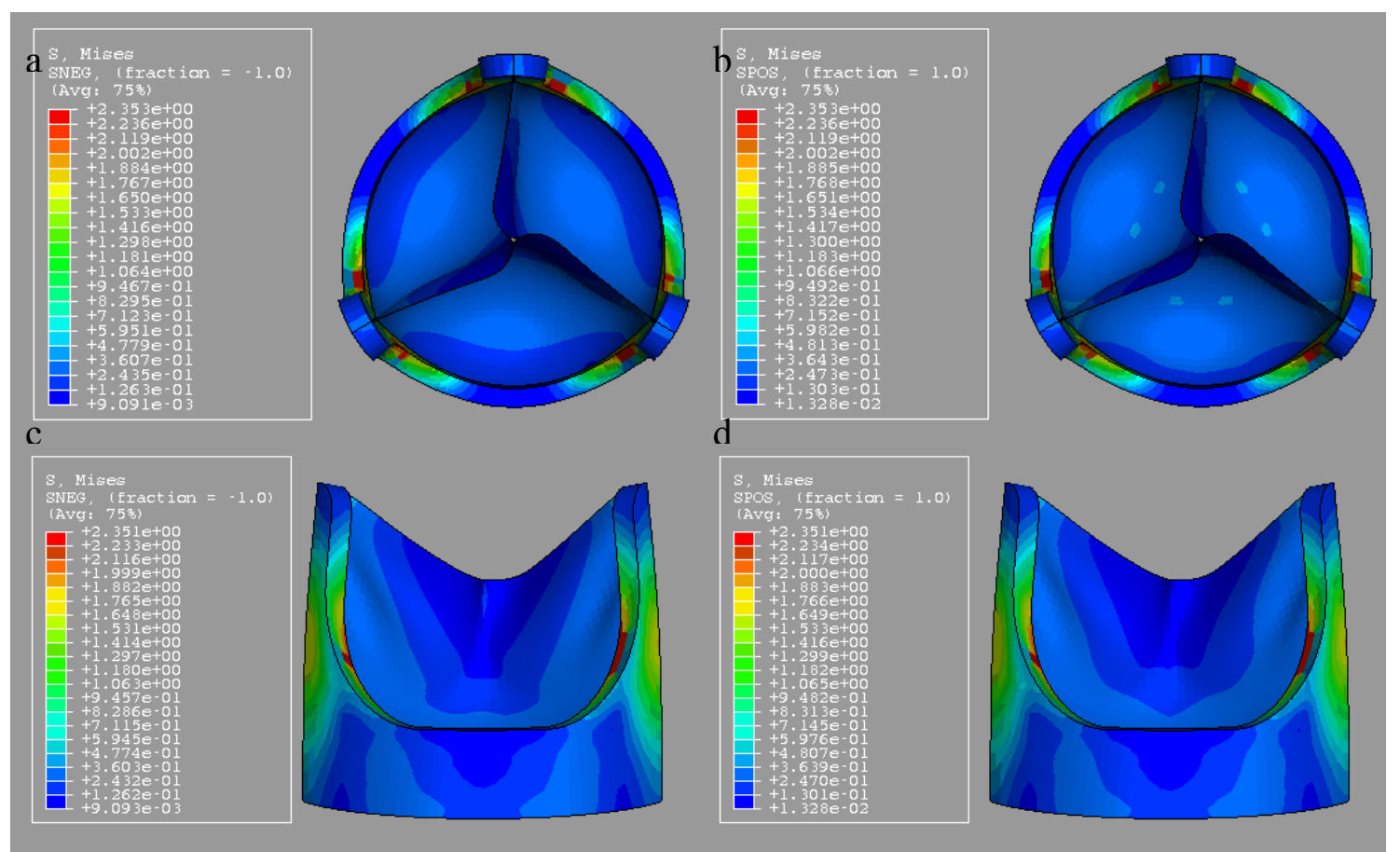

Figure 78. SIBS-leaflet with slight coaptation curvature mounted on a $48.5 \%$ styrene, medium profile stent (SIBS-Reg). Views include (a) top view of aortic surface stresses, (b) top view of ventricular surface stresses, (c) front view of one third of the valve showing aortic surface stresses, and (d) front view of one third of the valve showing the ventricular surface stresses.

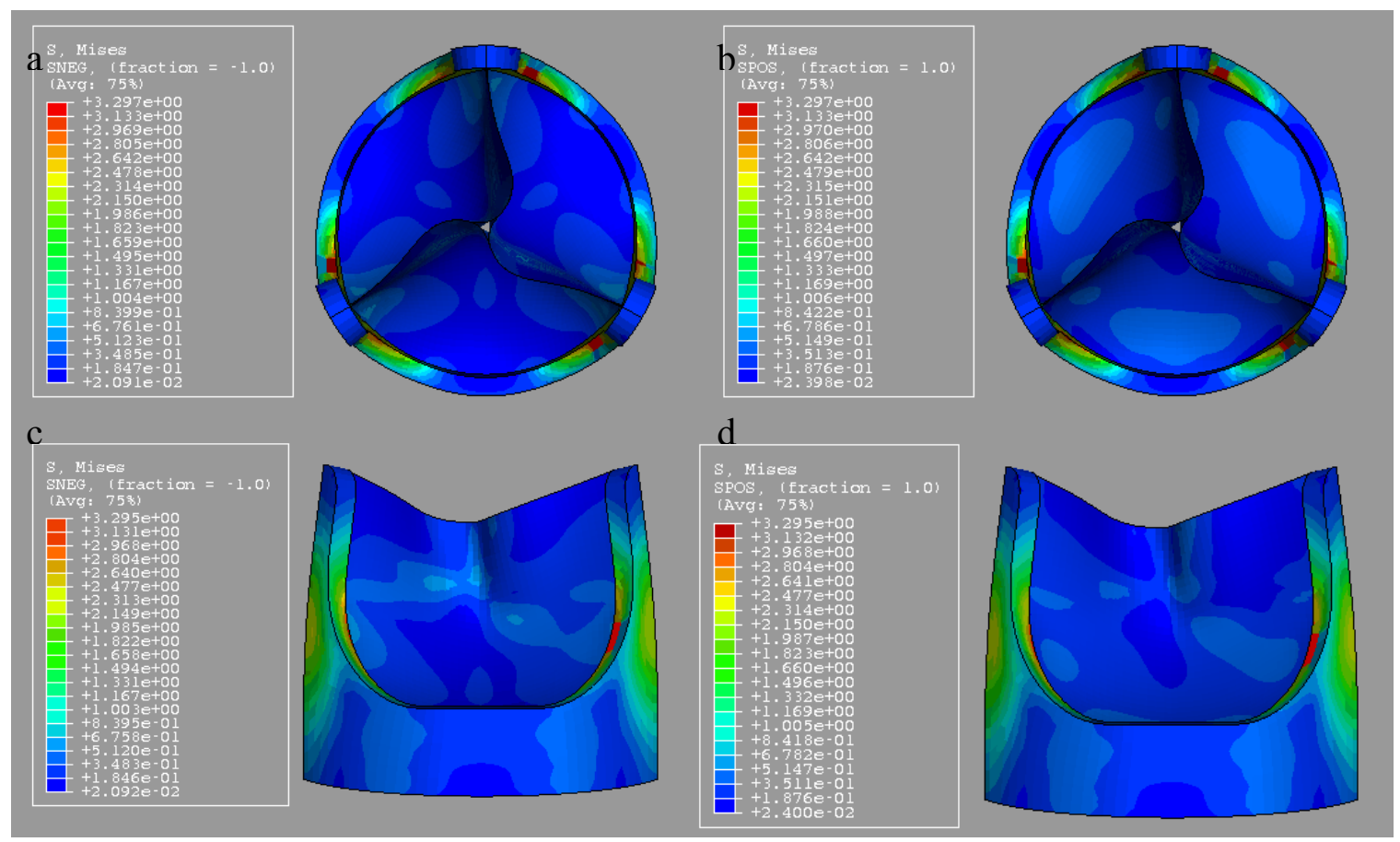

Figure 79. JSI-reinforced valve with slight coaptation curvature mounted on a $48.5 \%$ styrene, medium profile stent (JSI-Reg). Views include (a) top view of aortic surface stresses, (b) top view of ventricular surface stresses, (c) front view of one third of the valve showing aortic surface stresses, and (d) front view of one third of the valve showing the ventricular surface stresses. 


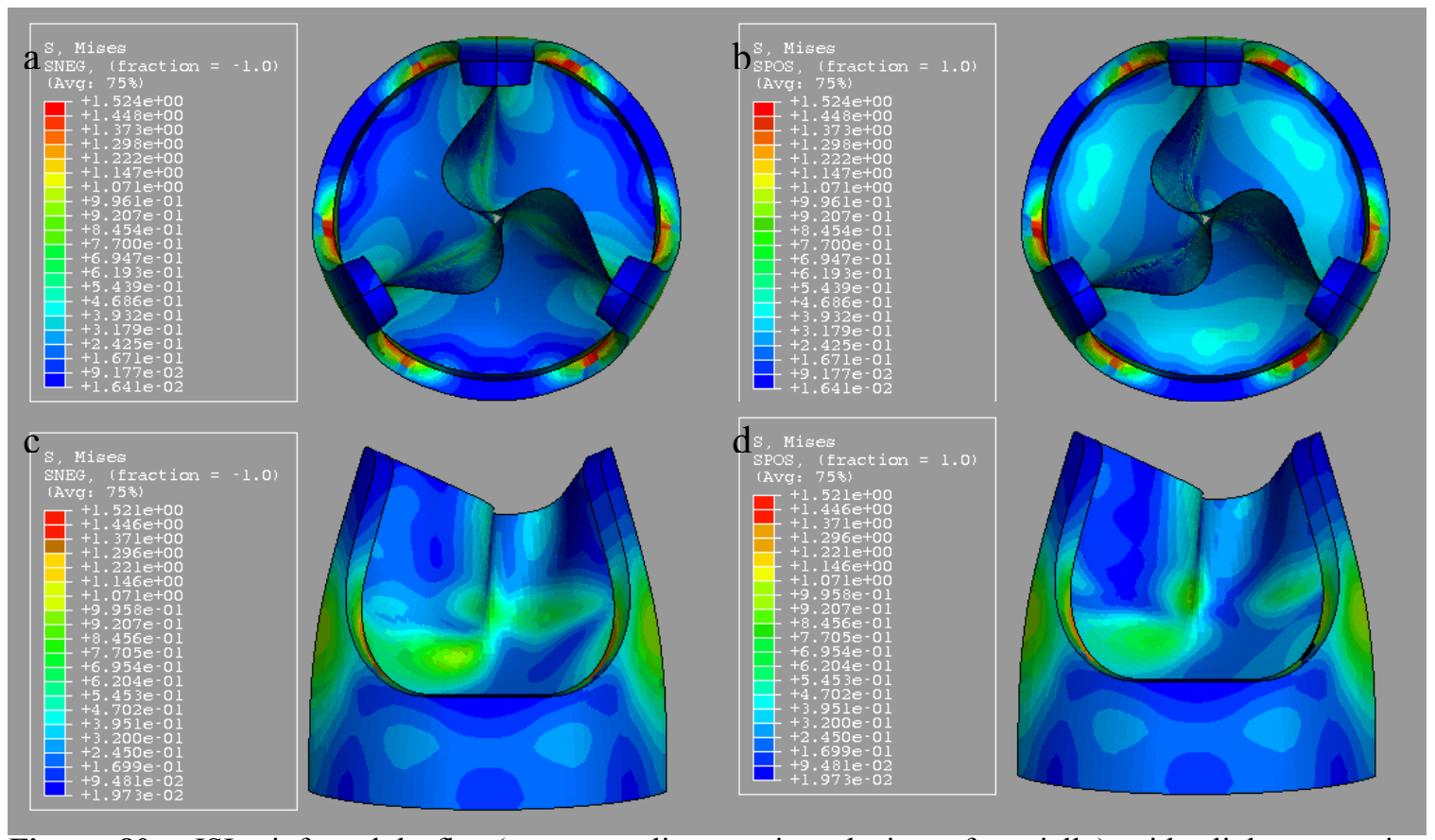

Figure 80. JSI-reinforced leaflet (max compliance oriented circumferentially) with slight coaptation curvature mounted on a 30\% styrene, high profile stent (JSI-HiP-Circ). Views include (a) top view of aortic surface stresses, (b) top view of ventricular surface stresses, (c) front view of one third of the valve showing aortic surface stresses, and (d) front view of one third of the valve showing the ventricular surface stresses.

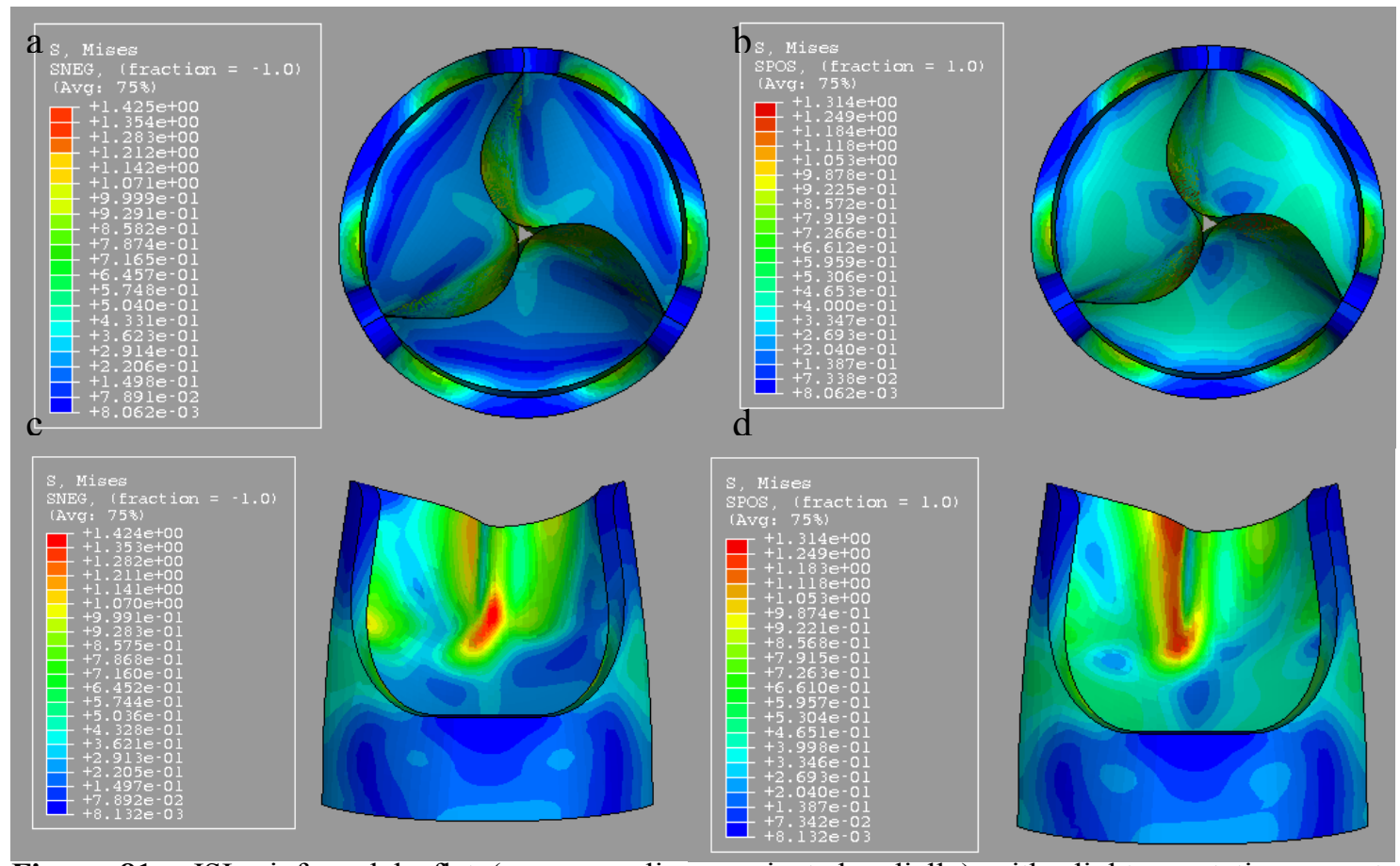

Figure 81. JSI-reinforced leaflet (max compliance oriented radially) with slight coaptation curvature mounted on a $30 \%$ styrene, high profile stent (JSI-HiP-Rad). Views include (a) top view of aortic surface stresses, (b) top view of ventricular surface stresses, (c) front view of one third of the valve showing aortic surface stresses, and (d) front view of one third of the valve showing the ventricular surface stresses. 


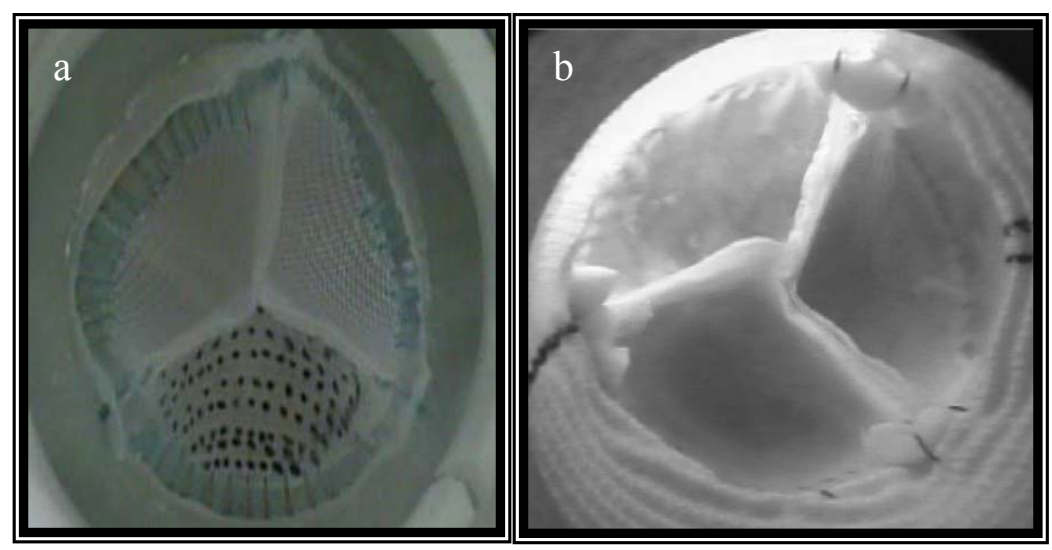

Figure 82. Images showing the coaptation interaction during diastole for a (a) BARD-reinforced leaflet mounted on a $48.5 \%$ styrene stent and a (b) bioprosthetic valve adapted from Labrosse et al. [65].

The high profile JSI valves (Figure 80 and Figure 81) appeared to have an increase in leaflet stress concentrations when compared with the lower profile version (Figure 79). Not only was the valve geometry different in this case, but a lower percentage styrene SIBS was used to manufacture the stents. The combination of a higher stent profile and a polymer with decreased stiffness resulted in more stent deformation. When the orientation of the dip-coated JSI fabric was switched to allow more radial compliance, a large difference to the leaflet and stent stress concentrations occurred. With more radial compliance, the leaflet stress increased, and a peak stress was evident in the mid-leaflet region, denoted by the red color (Figure 81). For all other valve models, the peak stress always occurred on the stent itself, and not the leaflet. When comparing the two high profile valves, the valve with the circumferential compliance had greater stent deformation than that of the valve with the radial compliance. This occurrence can be attributed to the fact that greater circumferential stiffness results in reduced leaflet deformation that is translated to the stent; therefore, the load on the stent is reduced. The resultant reduction in stress on the stent is not sufficient to warrant a 
change in the preferred reinforcement orientation (i.e. more circumferential compliance) as the large increase in stress that occurred in the leaflet itself ultimately led to the valve failure during in vitro fatigue. The location of the peak stress in the model is consistent with the location of hole formation in the same valve subjected to in vitro fatigue (Figure $83)$.

Based on the results presented above, the primary regions of concern in terms of elevated stress concentrations in the leaflets include the top of the commissure, lower commissure, center of coaptation, and belly region (Figure 84). For the higher profile valve, an additional region of high stress arose between the center of coaptation and belly region. The peak stresses in these five areas were compared between valves to assess how the different designs affect the overall stress concentrations within the valve. It should be noted that the peak stresses for the fiber-reinforced orthotropic models were compared with their experimental stress-strain curves, and each was determined to be within the elastic range of the material. The assumption of a purely elastic model is, therefore, valid for this analysis.

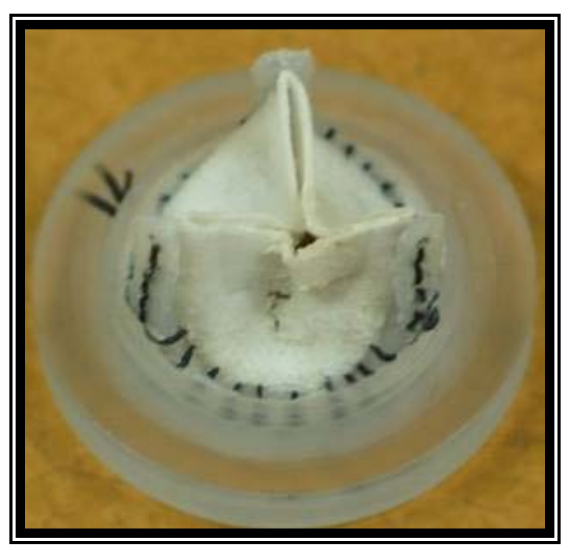

Figure 83. Dip-coated JSI-reinforced, high profile valve showing hole formation at the mid-valve region of cycling equivalent to 6.12 years 


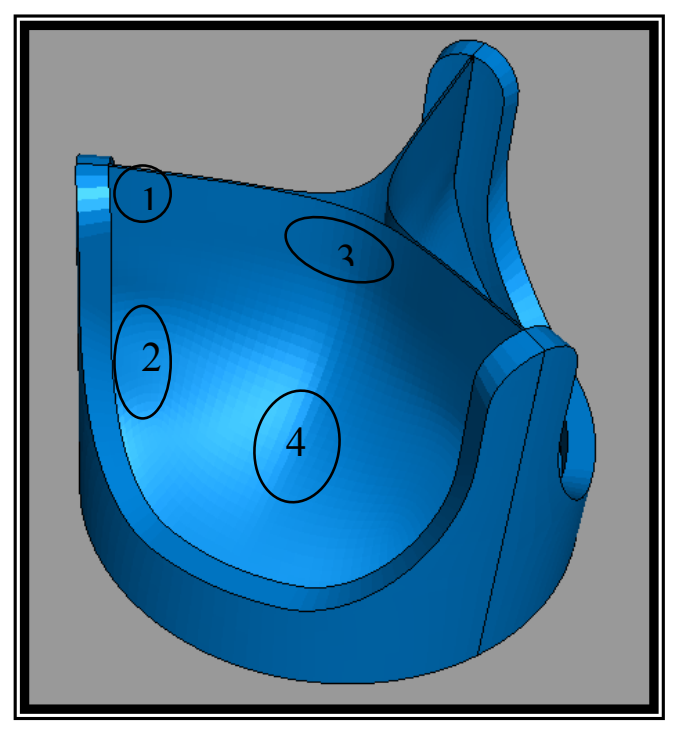

Figure 84. Critical areas of interest when comparing the leaflet stress concentrations, where (1) is the top of the commissure, (2) is the lower commissure, (3) is the center of coaptation, and (4) is the belly. For the higher profile valves, a fifth region defined as the mid valve was added between regions 3 and 4 .

A summary of the peak stresses for the four primary leaflet regions is presented in Figure 85. An ANOVA was run for each of these regions to assess whether any particular design resulted in a significantly larger stress concentration. For the top of the commissure, the three JSI-reinforced models were found to result in a significantly lower stress concentration than any of the other valve models, but they were not significantly different from each other $(\mathrm{p}=0.907)$. The BARD-reinforced design mounted on the rigid stent was found to result in a significantly larger stress concentration than any of the other models. What was interesting to note was that the three different BARD-reinforced valves with varying coaptation geometry were not found to result in significantly different stress concentration at the top of the commissure $(p=0.979)$. This is contrary to what has been presented in the literature, where stress concentrations at the commissure are believed to be affected by leaflet anisotropy, stent flexibility, and the angle the free edge forms with the stent post $[21,29,30,37-39,44,41,52,53]$. By changing the leaflet 
coaptation geometry, the angle between the free edge and stent post is varied, but in the case of the BARD-reinforced leaflet, it did not have any impact on the stress concentrations. Changing the stent flexibility did have a large impact, where increased flexibility resulted in a decreased stress for the BARD-reinforced valve.

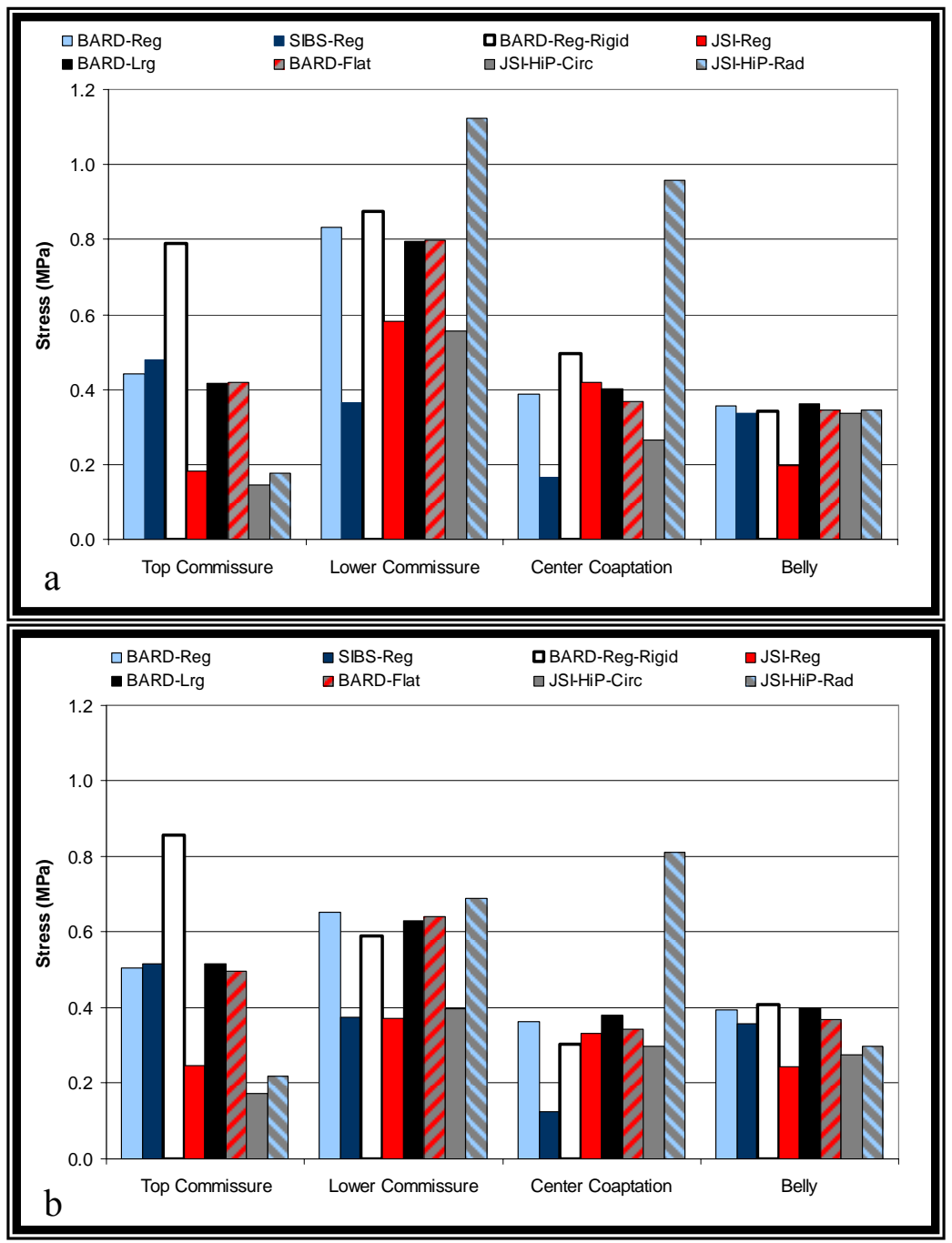

Figure 85. Peak stress concentrations for regions $1-4$ of all models at the (a) aortic and (b) ventricular surface.

When the stress concentrations for the lower commissure were compared, no significant difference was established between models $(\mathrm{p}=0.115)$. This is surprising 
when the values on the aortic surface are compared (Figure 85), but both the aortic and ventricular values were input into SPSS, and for some models, a large difference between the two existed. This would have resulted in a larger standard deviation, so when different models were compared, significance was not established.

On comparison of the stresses at the center of coaptation, the radially compliant, JSI-reinforced valve mounted on the high profile stent was found to have a significantly larger peak stress than any of the other models. The rest of the models were not found to be significantly different $(\mathrm{p}=0.60)$. It should be noted that all of the other models, excluding the isotropic SIBS model, had a lower circumferential modulus than radial modulus. The radially compliant valve mimics the general tendencies of the natural valve in which the circumferential modulus is higher than in the radial direction (refer to Figure 40); however, the circumferential modulus for the JSI-reinforced valve with radial compliance is significantly higher than that of the natural valve $\left(\mathrm{E}_{\mathrm{JSI}}=14.5 \mathrm{MPa}, \mathrm{E}_{\text {natural }}=\right.$ 5.79 MPa) [53]. The elevated stress in the radially compliant valve can be attributed to the fact that the increased modulus along the circumferential direction resulted in elevated stresses on the valve in order to achieve valve coaptation. For the JSI-reinforced valve with circumferential compliance, the circumferential modulus was closer to that of the natural valve $\left(\mathrm{E}_{\mathrm{JSI}}=2.97 \mathrm{MPa}, \mathrm{E}_{\text {natural }}=5.79 \mathrm{MPa}\right)$, even though the radial moduli were considerably different $\left(\mathrm{E}_{\mathrm{JSI}}=14.5 \mathrm{MPa}, \mathrm{E}_{\text {natural }}=0.966 \mathrm{MPa}\right)$. Orienting the JSI reinforcement in order to have closer matching of circumferential modulus with the natural valve appeared to be more beneficial in terms of stress reduction than replicating the circumferential versus radial compliance tendencies. 
In terms of the stress concentrations in the belly region, once again the three JSIreinforced valves were not found to be significantly different from each other $(p=0.136)$, but the JSI-reinforced leaflets mounted on the $48.5 \%$ styrene stent were found to have a significantly lower stress concentration than any of the other valves mounted on the $48.5 \%$ styrene stent.

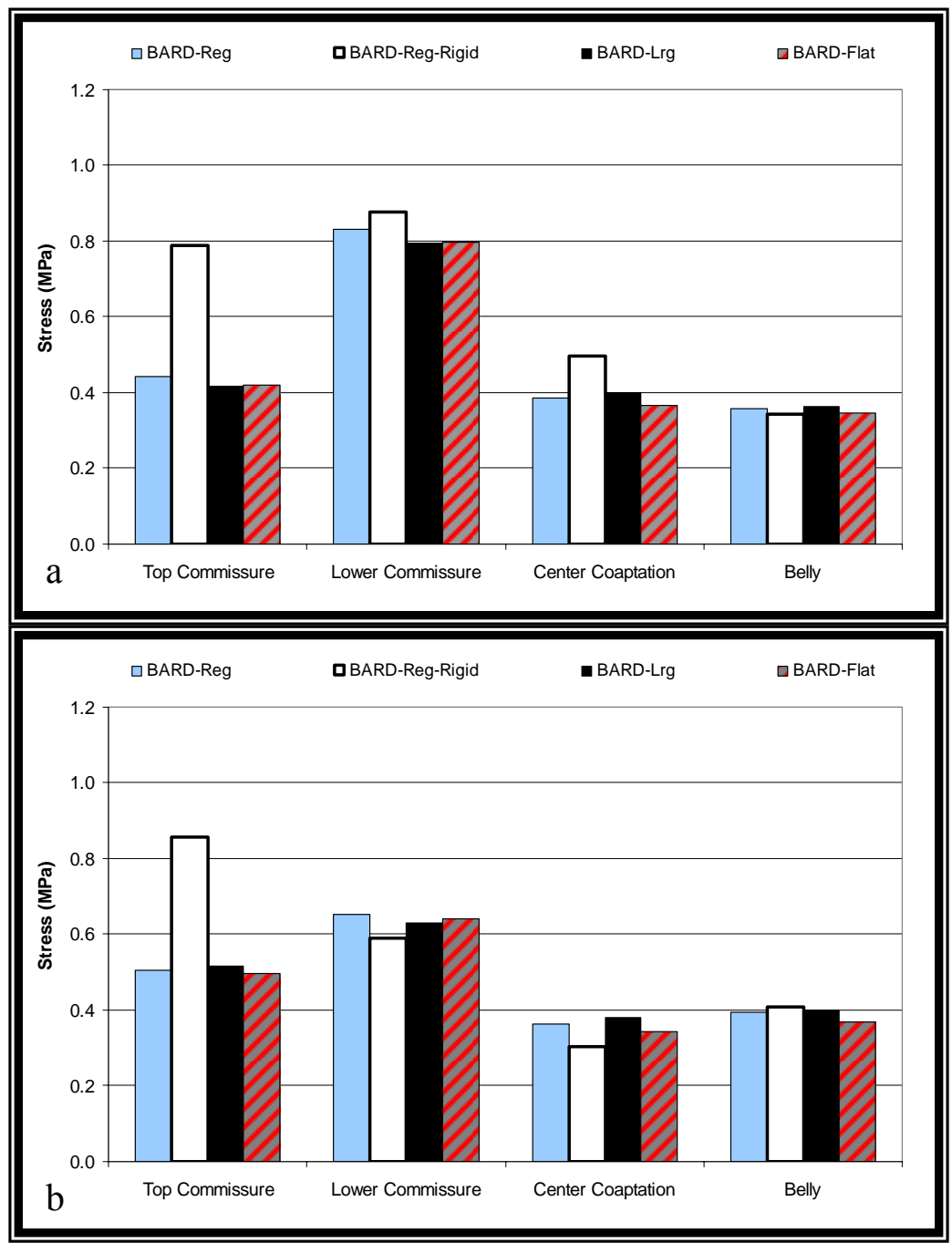

Figure 86. Peak stress concentrations for regions $1-4$ for all BARD valve models at the (a) aortic and (b) ventricular surface. 
In order to further investigate the impact of varying valve designs on leaflet stress concentrations, the results were grouped in terms of leaflet reinforcement fabric and stent design. When comparing the output for the three BARD-reinforced valves with varying coaptation geometries, the statistical analysis stated that none of the stress concentrations could be considered to be significantly different, and this is made more apparent in Figure 86. One can then conclude that the coaptation geometry in the range of what was tested does not significantly affect the stress concentrations in the leaflet, so the most favorable geometry would be that which results in the least percent regurgitation during hydrodynamic evaluation.

For the BARD-reinforced valve, increasing the stiffness of the supporting stent led to a significant increase in the stress concentration at the top of the commissure (refer to Figure 86). It did not, however, have a large impact on the stresses in other regions of the leaflet. From this, one can conclude that a more flexible stent is favorable for reduced commissure stresses, but based on previous in vivo studies, a balance must be found. If the stent is too flexible, it can be susceptible to excessive buckling, and if it is too stiff, it can cause increased leaflet stress and a resultant decrease in leaflet durability. According to Cacciola et al. [31], rigid supporting stents increase the degree of leaflet flexure, and this can cause an increase in the leaflet stress concentrations. In order to investigate this, Cacciola et al. [31] performed an evaluation of stentless versus stented valves, including leaflets with identical material properties, and what was found was that the stentless formulation did in fact result in a $75 \%$ reduction of stress. Stentless valves do differ from flexible stented valves, but the allowance of leaflet boundary displacement is similar, thereby reiterating the benefits to supporting structure flexibility. 


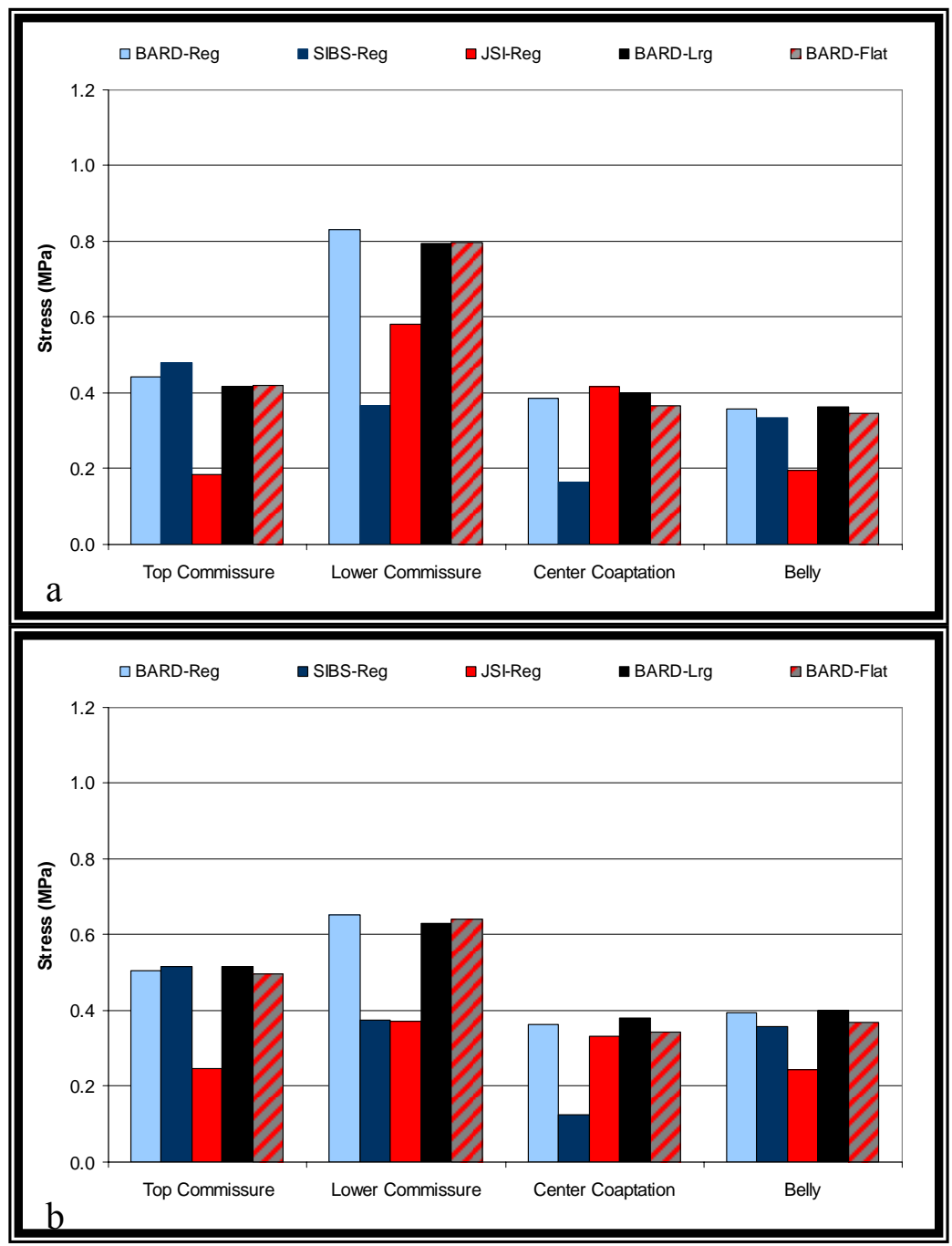

Figure 87. Peak stress concentrations for regions $1-4$ for all valve models including a $48.5 \%$ styrene at the (a) aortic and (b) ventricular surface.

For the leaflets mounted on the $48.5 \%$ styrene, medium profile stent, statistical significance was established for the JSI-reinforced leaflet at the top of the commissure and in the belly region. In both of these regions, the JSI valve had a significantly lower stress concentration than either the BARD-reinforced or isotropic SIBS leaflets (refer to Figure 87). Analysis of the tensile properties of the BARD and JSI leaflet materials established that their material properties along the radial and circumferential directions were not significantly different. The materials do differ in thickness, where the JSI 
leaflet is double the thickness of the BARD leaflet $(0.40 \mathrm{~mm}$ to $0.20 \mathrm{~mm}$, respectively). One can conclude that a thicker leaflet material aids in the reduction of stress during diastolic loading; however, the hydrodynamic evaluation already established that the thicker JSI leaflet resulted in a significantly higher transvalvular pressure drop during forward flow, so a thinner leaflet is more favorable for hydrodynamic function. According to Deck and Thubrikar et al. $[73,75]$ a decrease in the leaflet thickness results in an increase in the tensile stresses within the leaflet. In their evaluation of natural, bioprosthetic, and synthetic leaflet valves, they calculated membrane stress according to the equation: membrane stress $=\mathrm{PR} / \mathrm{t}$, where $\mathrm{P}$ was the pressure difference across the leaflet, $\mathrm{R}$ was the radius of the leaflet, and $\mathrm{t}$ was the leaflet thickness. It can be seen in this formulation that leaflet thickness and tensile stress were inversely proportional, resulting in an increased stress with a decrease in leaflet thickness.

During comparison of the different JSI-reinforced valve designs, statistical significance was only established for the radially oriented version mounted on the high profile, $30 \%$ styrene stent. On comparison of the two different circumferentially oriented designs (Figure 88), the valve mounted on the more flexible stent appeared to have a reduced stress concentration at the top of the commissure and the center of coaptation, and it had a higher stress concentration in the belly region. At the lower commissure, there was a different trend on the ventricular surface when compared with the aortic surface. Significance was not established between these designs, so one cannot conclude that there is any benefit, in terms of stress minimization, to a higher profile and more flexible stent. This is somewhat contradictory to what was established for the BARDreinforced valve; therefore, it can be concluded that an increase in flexibility does have 
its limitations. An increase from completely rigid to partially flexible is beneficial, but when the flexibility increases to the point of resulting in large stent post deformation (Figure 80 ), the positive effects are reduced. When the highly flexible valve was implanted in an in vivo sheep model, severe stent deformation occurred resulting in valve incompetence $[12,15]$. There is, therefore, no added benefit to highly flexible stents.

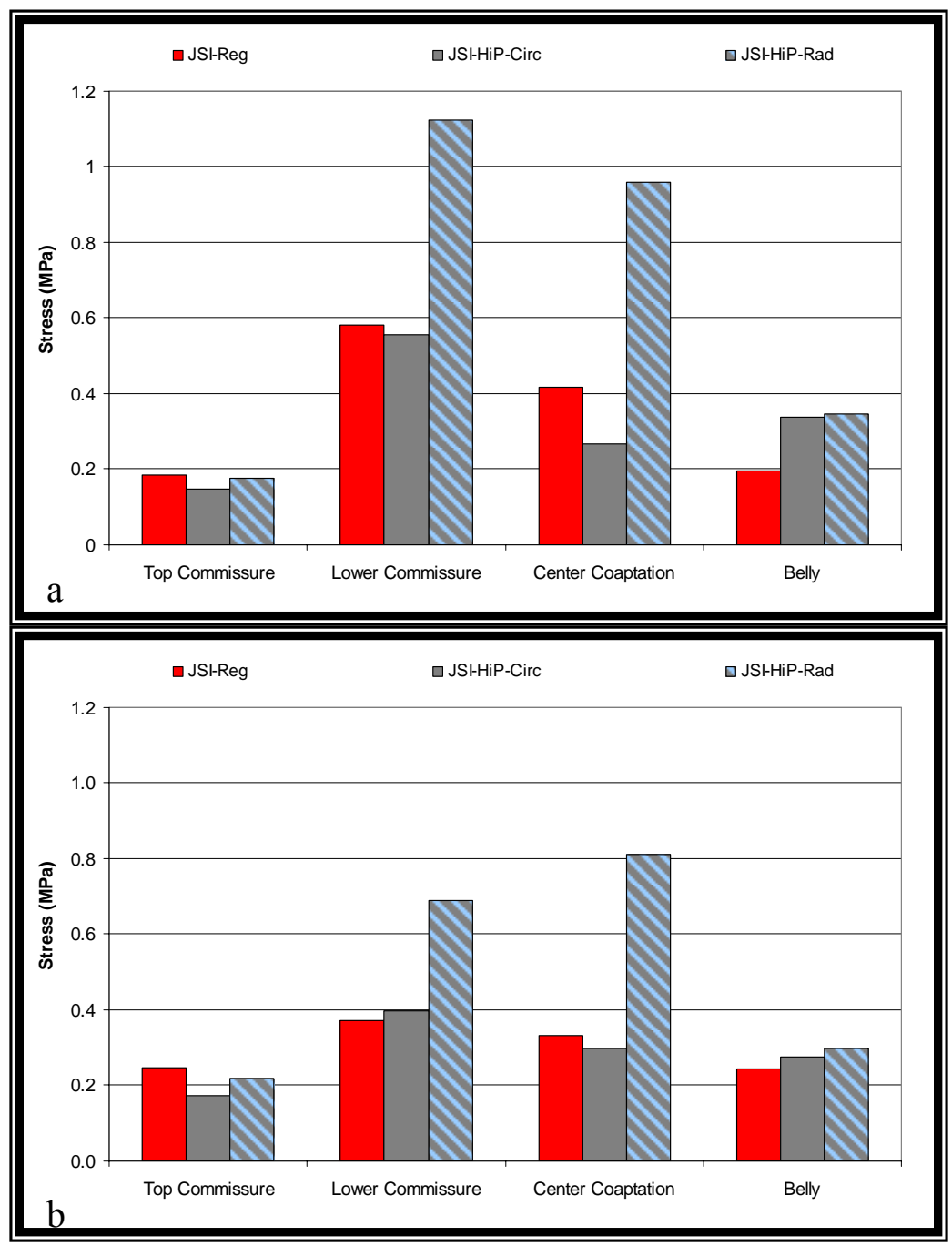

Figure 88. Peak stress concentrations for regions $1-4$ for all JSI-reinforced valve models at the (a) aortic and (b) ventricular surface. 
With the quasi-static loading of the leaflet, the change in stress with time was of interest. The plots of stress versus time for the four primary leaflet regions of interest (Figure 89 -Figure 92) reaffirm what is already known about which valves result in a significantly higher or lower stress concentration. Interesting points of note occur at the lower commissure, center of coaptation, belly region, and at the mid-valve. At the lower commissure, the JSI-reinforced valve with radial compliance and a highly flexible stent has a stress concentration in the region of all the other valves at a low pressure load, but after 0.03 seconds, an abrupt increase in the stress concentration occurs, resulting in it having the highest peak stress at the maximum diastolic load. At the center of coaptation, this same valve displays an abrupt increase in stress, and then the stress appears to plateau until the maximum pressure load is reached. When the two high profile valves are compared in terms of their stress concentrations in the mid-valve region, the radially oriented JSI valve starts out with a lower stress concentration in this region, but once again, after approximately 0.03 seconds, a sharp increase in the stress is seen. All other valves show a more gradual increase in their stress state over time.

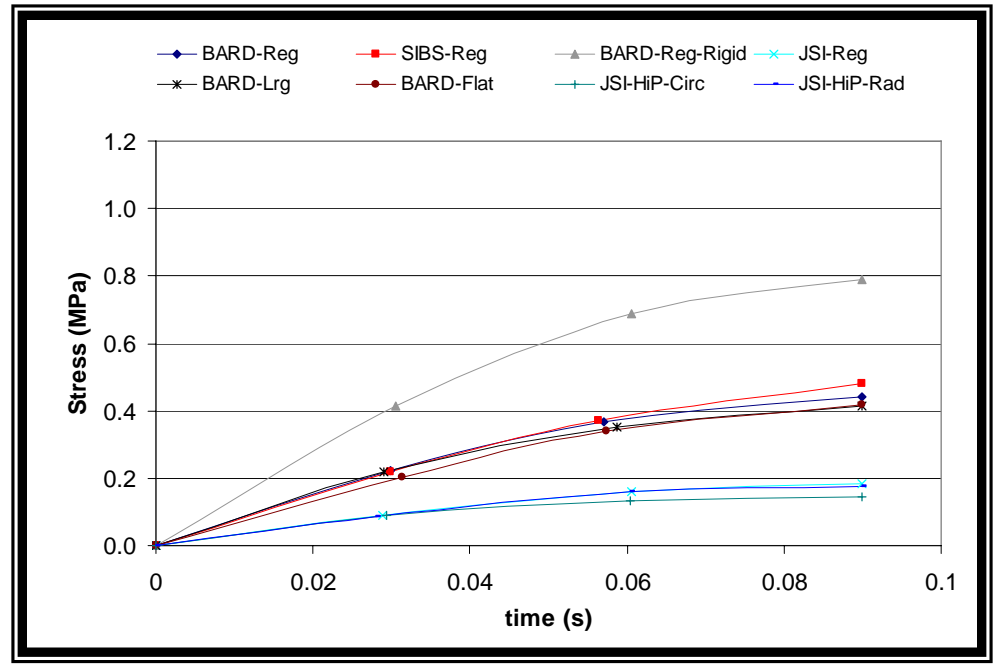

Figure 89. Peak stress concentrations for the aortic surface of all valves at the top of the commissure. 


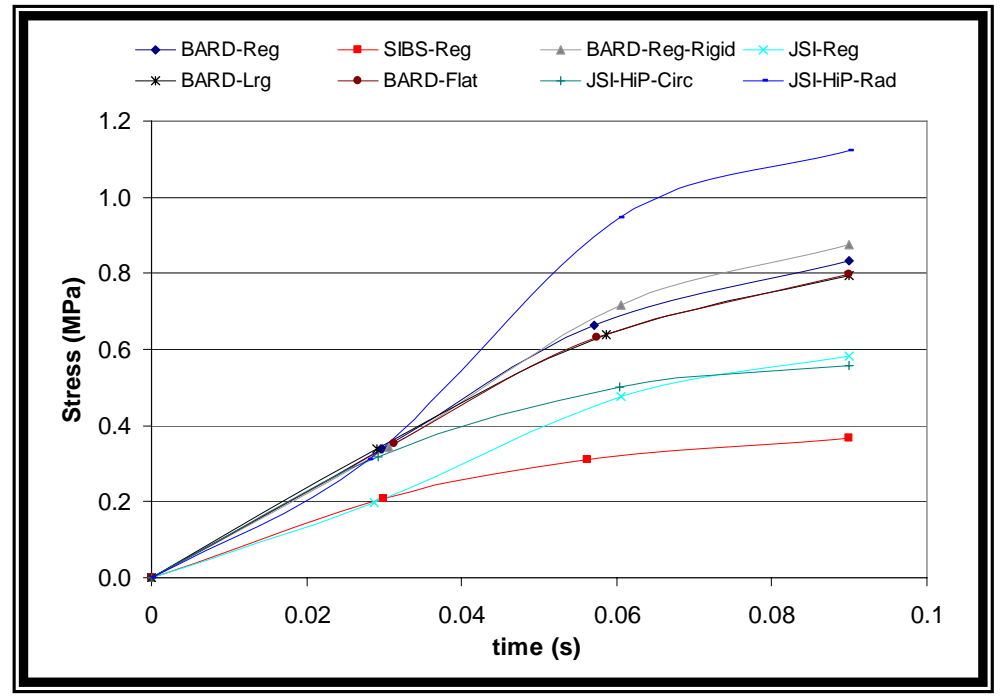

Figure 90. Peak stress concentrations for the aortic surface of all valves at the lower commissure.

The results from the two high profile JSI-reinforced valves showed that a reduction in stress occurred when the orientation of the reinforcement fabric was opposite to that of the natural valve: the natural valve has a lower modulus in the radial direction than in the circumferential direction. The difference in the high and low moduli between the natural and JSI valves were large, so questions arose as to whether it was more important to mimic the trend of greater radial compliance or whether it was more important to mimic the moduli. When using commercially available reinforcement materials, the particular properties of the prosthetic valve are restricted by what materials are available, so it becomes necessary to decide what characteristics of the natural valve are important to replicate in order to produce a prosthesis that has a reduced stress concentration and, therefore, increased durability. According to Thubrikar et al. [75], the principal stresses in the natural leaflet occur in both the circumferential and radial direction, but the tensile and flexural loading that the leaflet is subjected to predominates along the circumferential direction As a result, one may consider the properties and 
responses along the circumferential direction of the leaflet as more critical to the valve's function and longevity. Based on this information, an additional three high stent profile models were run to investigate this phenomenon. Two models included isotropic leaflets of either the high $(\mathrm{E}=14.5 \mathrm{MPa})$ or low $(\mathrm{E}=2.97 \mathrm{MPa})$ moduli of the JSI reinforcement, and the third model assumed the orthotropic moduli of a porcine heart valve $\left(\mathrm{E}_{\mathrm{circ}}=5.79\right.$ $\mathrm{MPa}, \mathrm{E}_{\mathrm{rad}}=0.966 \mathrm{MPa}$ ) obtained from Li et al. [53]. The graphical representations of the surface stress concentrations for the additional three models are provided in Figure 94 Figure 96. It is apparent that the higher modulus isotropic leaflet (Figure 94) displayed the same peak stress concentration in the center of the leaflet that the radially oriented JSI-reinforced valve (Figure 81) did. Both of these valves had the same circumferential modulus $(\mathrm{E}=14.5 \mathrm{MPa})$, but the radial modulus for the JSI valve was much lower $(\mathrm{E}=$ 2.97 $\mathrm{MPa}$ ) than that of the isotropic valve. The low modulus isotropic valve and the valve with the natural valve orthotropic material properties had a marked reduction in the peak stress concentration when compared with the high modulus isotropic valve.

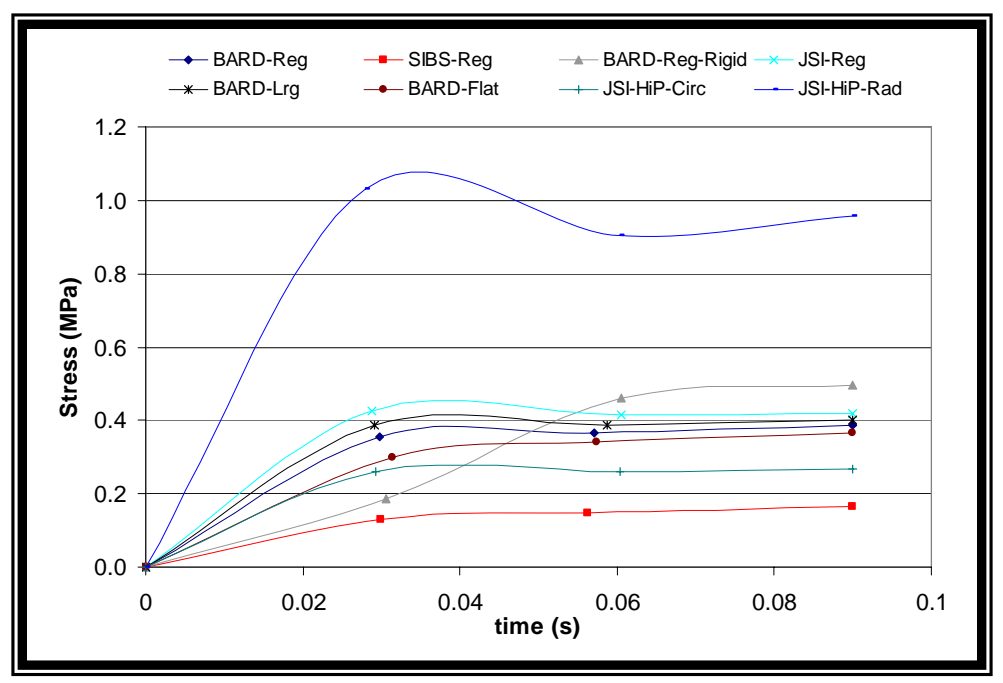

Figure 91. Peak stress concentrations for the aortic surface of all valves at the center of coaptation. 


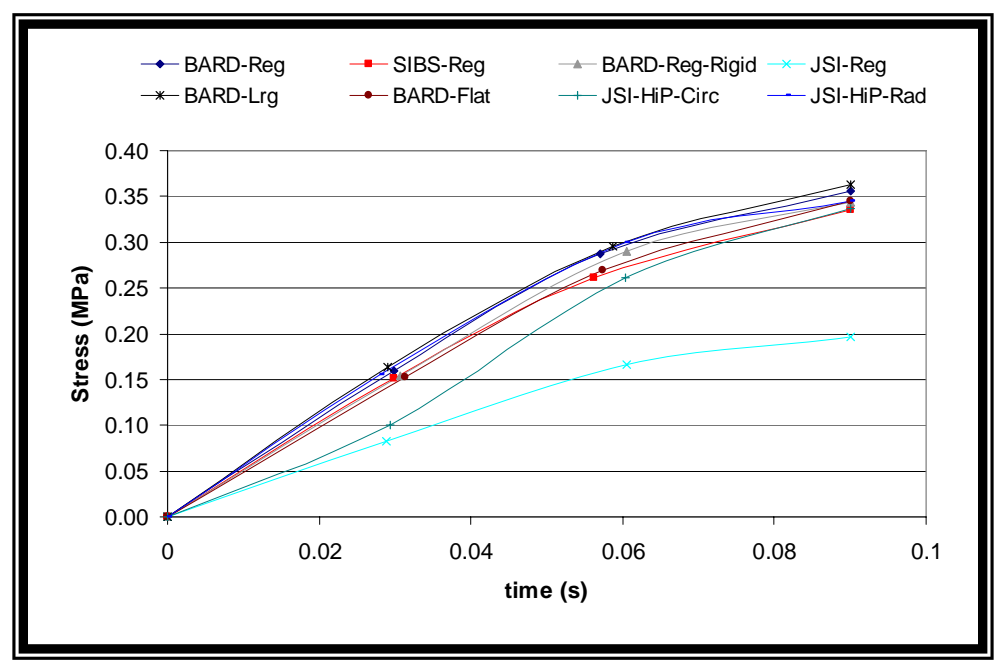

Figure 92. Peak stress concentrations for the aortic surface of all valves in the belly region.

On comparison of the peak stress concentrations between the three new models and the original three JSI-reinforced models (Figure 97) it is apparent that at the lower commissure and the center of coaptation, the two valves with increased circumferential modulus (radially oriented JSI and high modulus isotropic) show elevated stress concentrations.

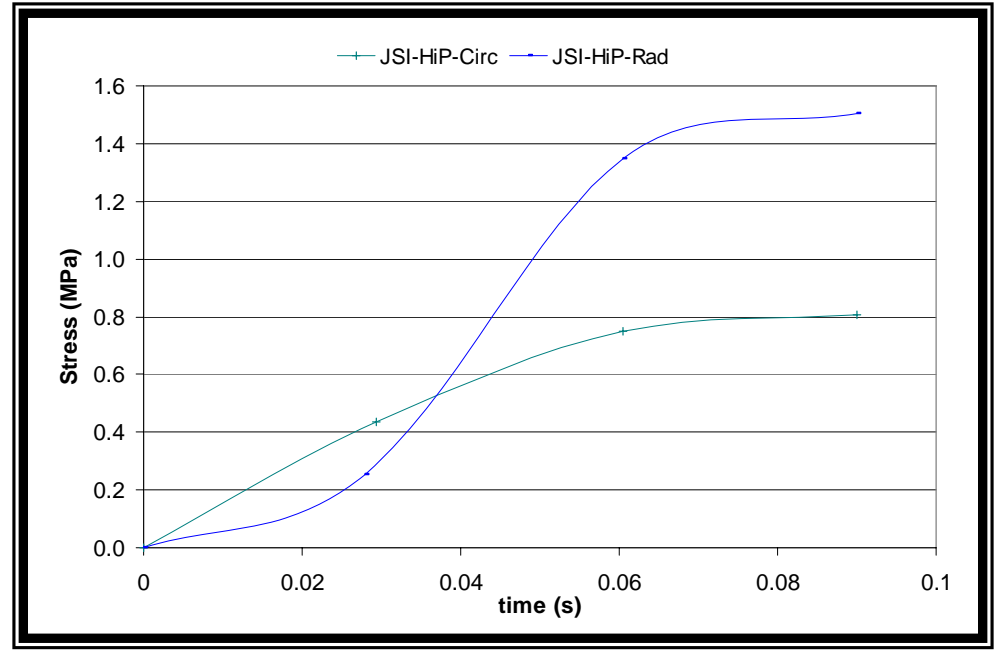

Figure 93. Peak stress concentrations for the high profile JSI-reinforced valves at the mid-valve region. 


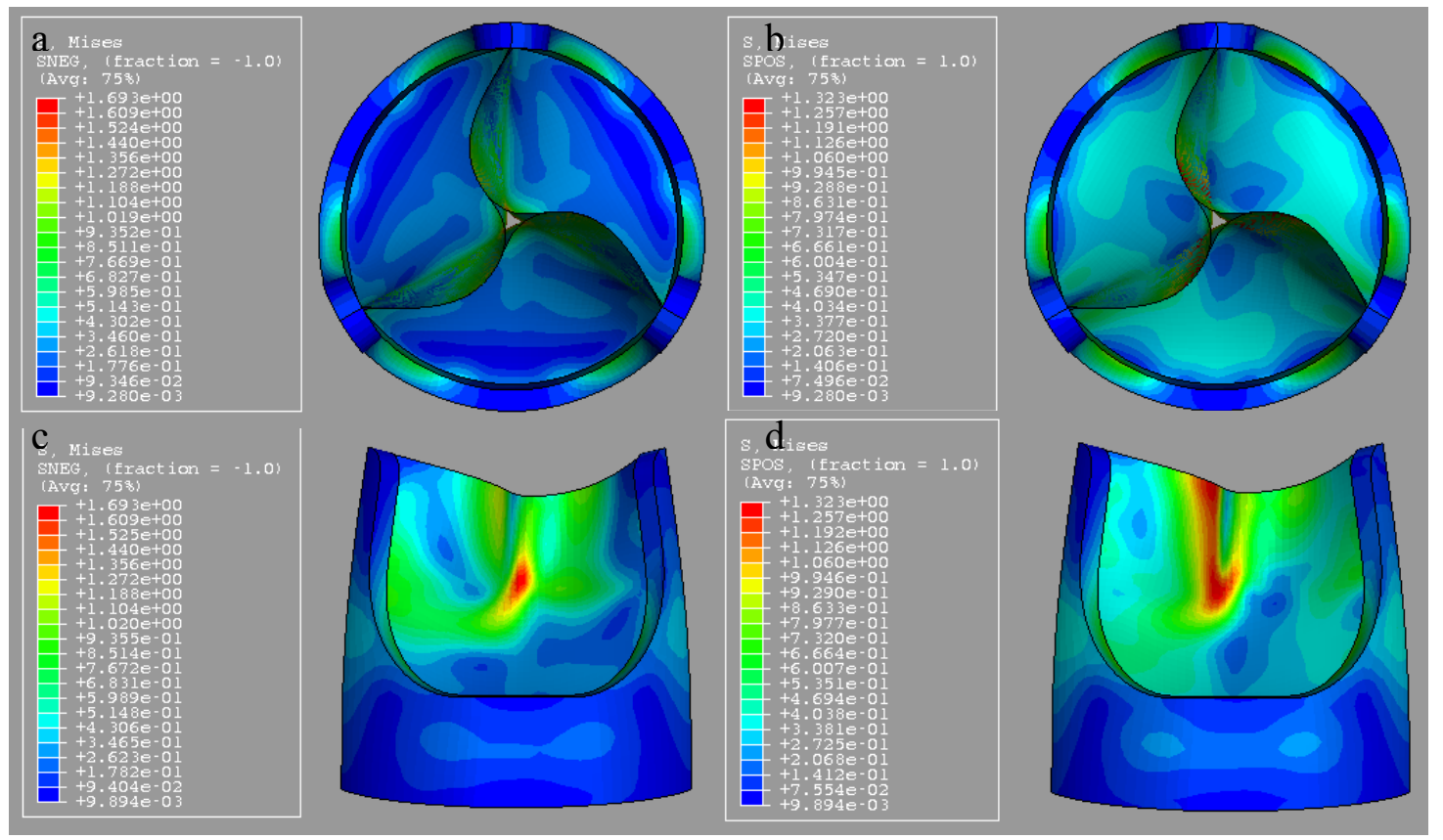

Figure 94. Isotropic leaflet with mechanical property mimicking that of higher modulus JSI-reinforced orientation $(\mathrm{E}=14.5 \mathrm{MPa})$ with slight coaptation curvature mounted on a $30 \%$ styrene, high profile stent (IsoHi-HiP). Views include (a) top view of aortic surface stresses, (b) top view of ventricular surface stresses, (c) front view of one third of the valve showing aortic surface stresses, and (d) front view of one third of the valve showing the ventricular surface stresses.
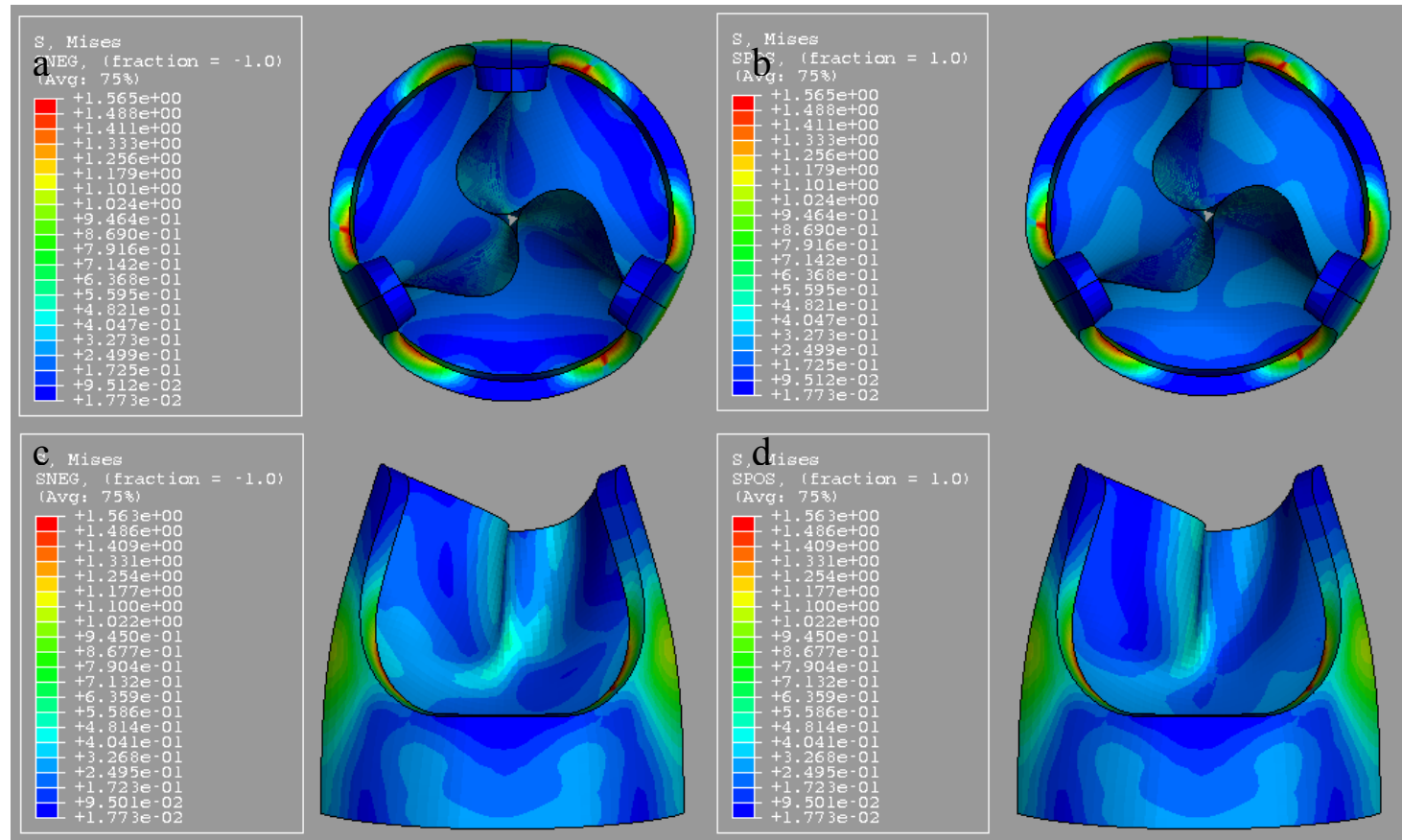

Figure 95. Isotropic leaflet with mechanical property mimicking that of lower modulus JSI-reinforced orientation $(E=2.97 \mathrm{MPa})$ with slight coaptation curvature mounted on a $30 \%$ styrene, high profile stent (IsoLo-HiP). Views include (a) top view of aortic surface stresses, (b) top view of ventricular surface stresses, (c) front view of one third of the valve showing aortic surface stresses, and (d) front view of one third of the valve showing the ventricular surface stresses. 
An ANOVA comparing the peak stress concentrations of the original eight models and the additional three established no significance at the top and lower commissure; however, at the center of coaptation, the low modulus isotropic valve, the circumferentially oriented high profile JSI valve, the circumferentially oriented medium profile JSI valve, and the natural orthotropic high profile valve were not significantly different. Both the isotropic high modulus valve and the radially oriented high profile JSI valve were found to have a significantly higher stress concentration than all other valves. In the belly region, the low modulus isotropic valve and the radially oriented JSI valve were found to be significantly different from each other, but they were not found to be different from any of the other valves.

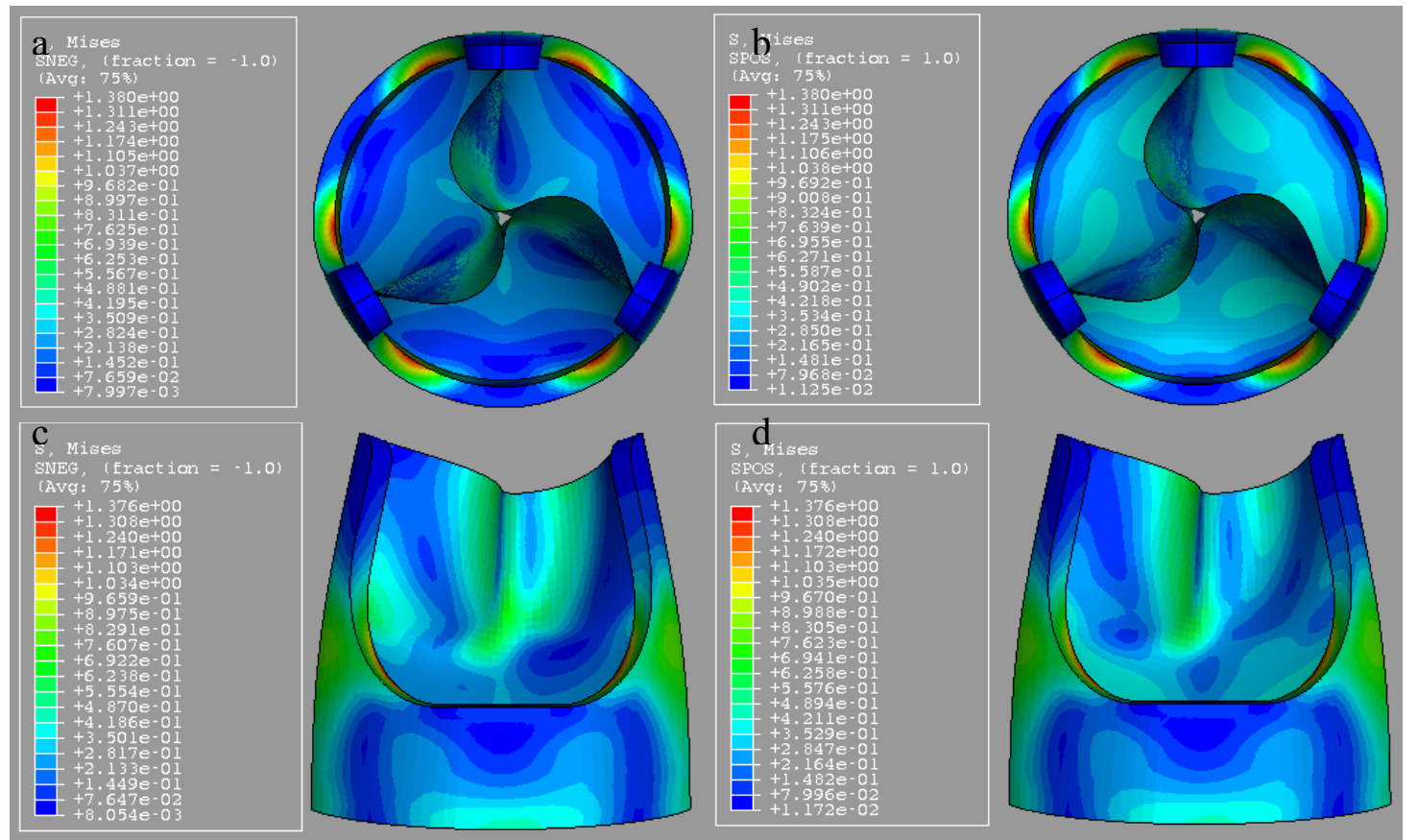

Figure 96. Orthotropic leaflet with mechanical property mimicking that the natural valve $\left(\mathrm{E}_{\text {circ }}=5.79\right.$ $\mathrm{MPa}, \mathrm{E}_{\mathrm{rad}}=0.966 \mathrm{MPa}$ ) with slight coaptation curvature mounted on a $30 \%$ styrene, high profile stent (Natural-HiP). Views include (a) top view of aortic surface stresses, (b) top view of ventricular surface stresses, (c) front view of one third of the valve showing aortic surface stresses, and (d) front view of one third of the valve showing the ventricular surface stresses. 


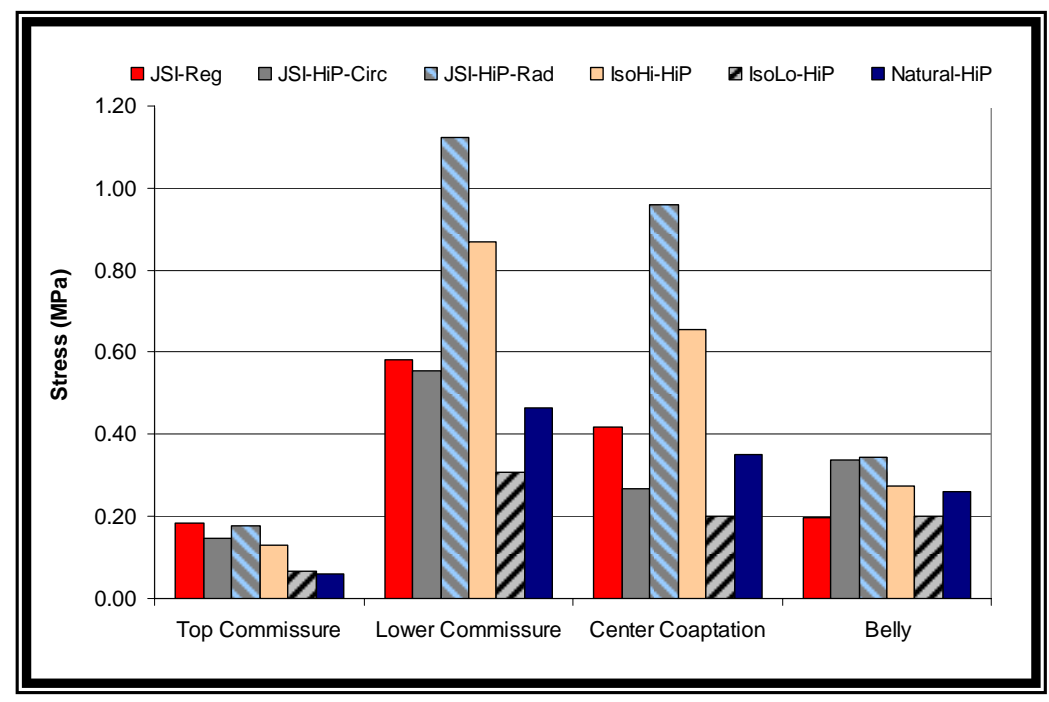

Figure 97. Peak stress concentrations for regions $1-4$ on the aortic surface for all JSI-reinforced valve models and the high profile models including the orthotropic natural valve properties and the isotropic $(\mathrm{E}=$ $2.97 \mathrm{MPa}$ and $\mathrm{E}=14.5 \mathrm{MPa}$ ) properties.

Based on this analysis, it appears that the two valves with the highest circumferential moduli resulted in the highest leaflet stress concentration. The circumferential modulus for both of these valves was identical, and it was markedly higher than that of the natural valve. This knowledge and the belief of Thubrikar et al. [75], that the valve is subjected to flexural and tensile stresses predominantly in the circumferential direction, prompted an evaluation of the component stresses in the circumferential and radial directions (Figure 98 - Figure 100). It is apparent from these figures that the stress along the circumferential direction is higher than that in the radial direction, across the board; therefore, one can conclude that the peak loads are oriented along the circumferential direction, and the properties in this direction are more critical for the minimization of stress in combination with increased valve longevity. What was found was that by reducing the circumferential modulus to provide a closer match to that of the natural valve, a reduction in the leaflet stress concentration could be obtained. 


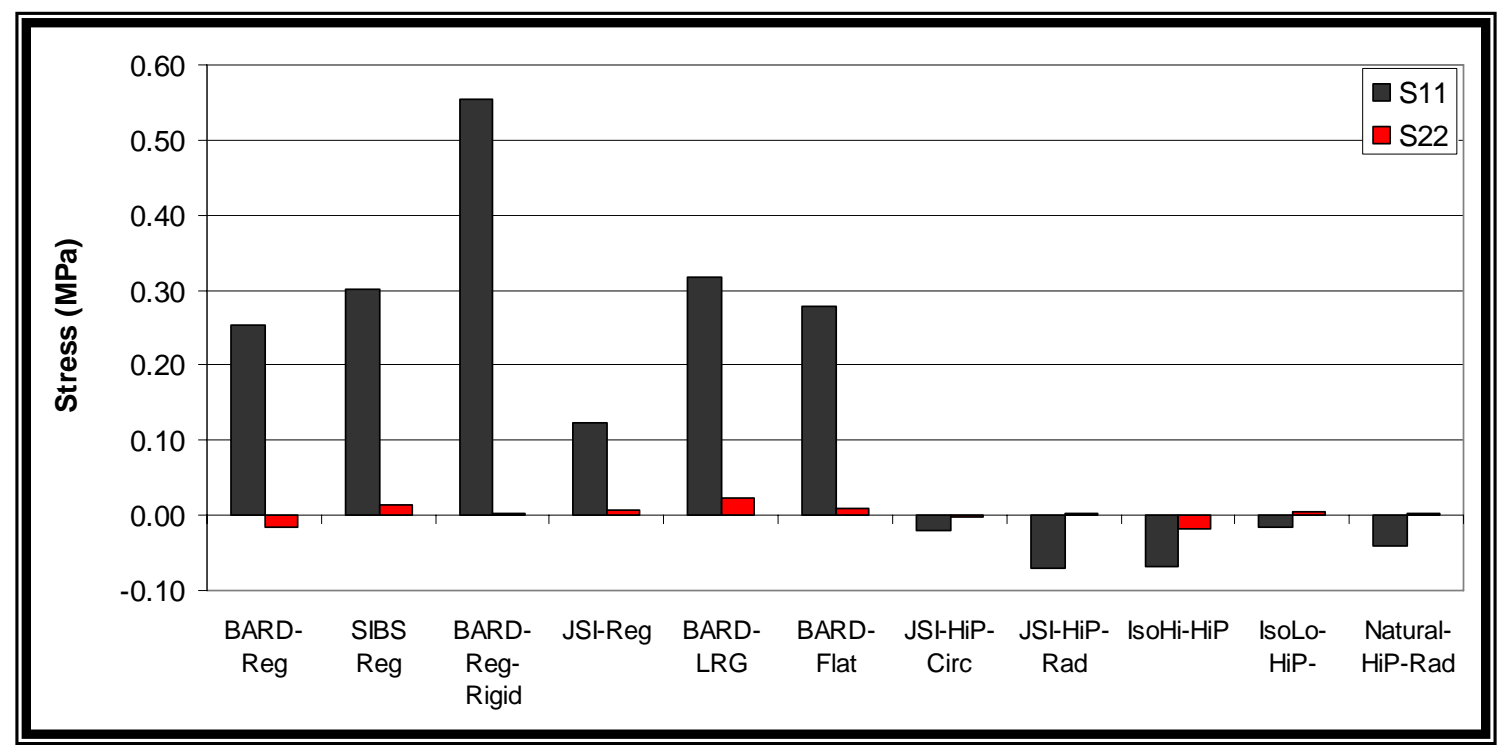

Figure 98. Component stress concentrations in the circumferential (S11) and radial (S22) directions for all models at the top of commissure.

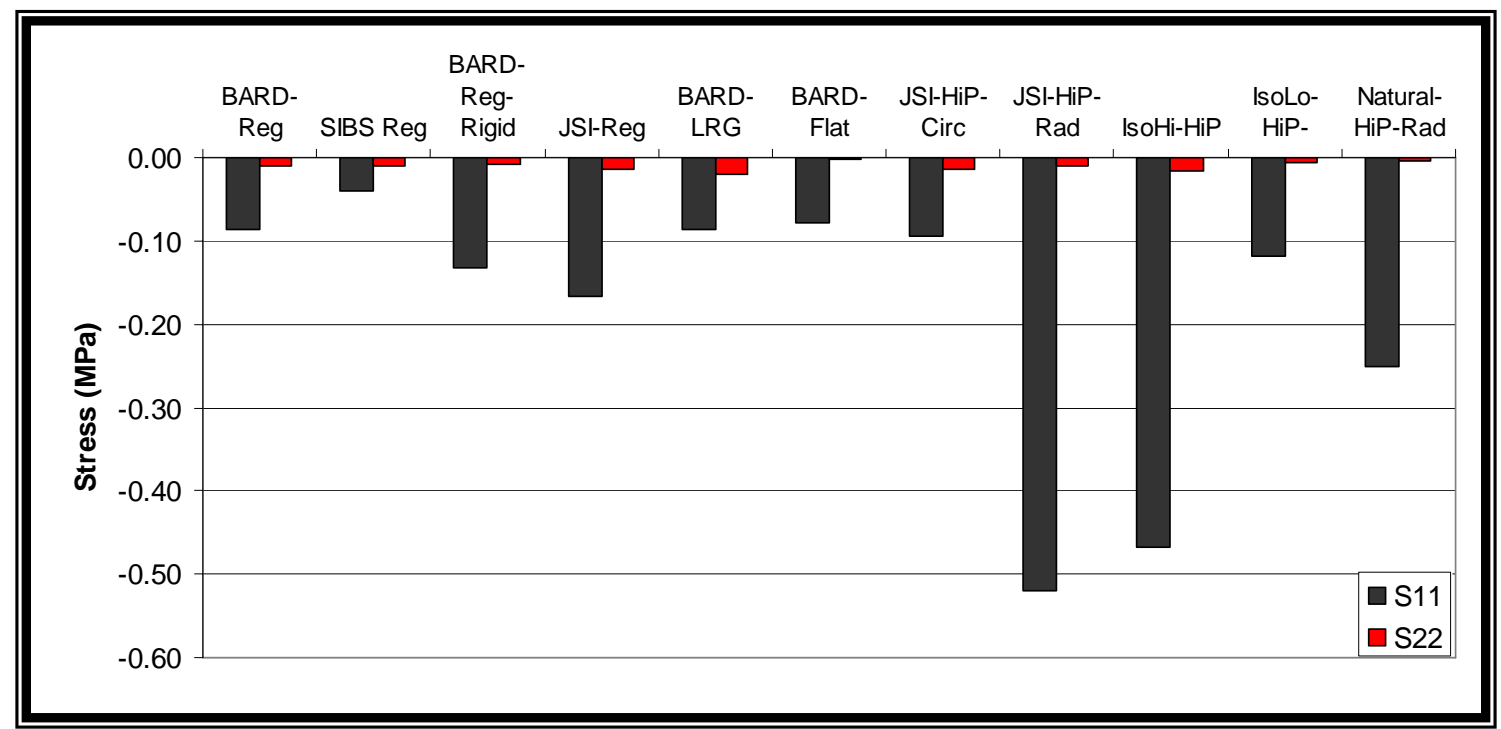

Figure 99. Component stress concentrations in the circumferential (S11) and radial (S22) directions for all models at the center of coaptation.

The stress/strain curves of the natural heart valve show an initial low modulus region followed by a high modulus region at elevated strains [75]. For the model of the natural valve represented in Figure 96, a constant Young's modulus was assumed according to Li et al. [53]. This simplification allowed a direct comparison between the 
SIBS valve models and the natural valve leaflet as they represented the same orthotropic nature.

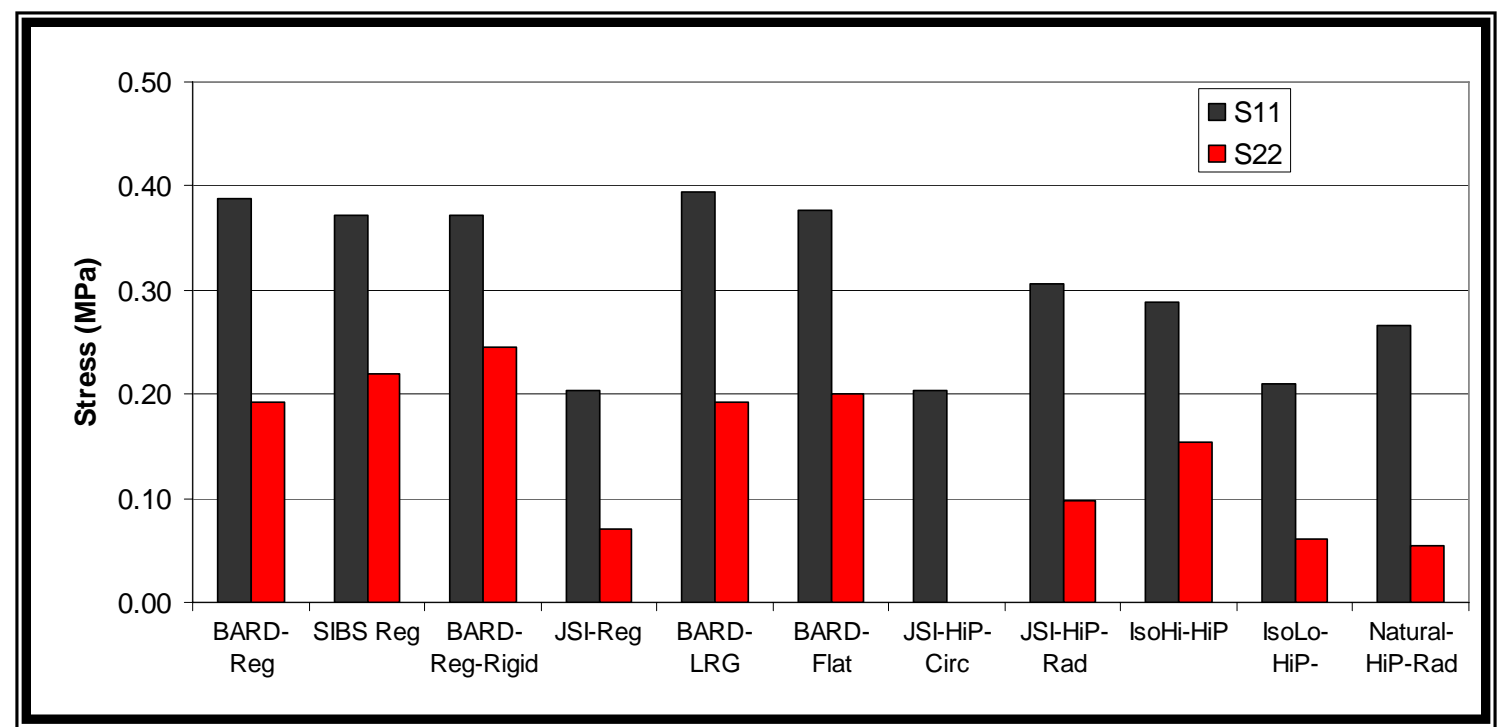

Figure 100. Component stress concentrations in the circumferential (S11) and radial (S22) directions for all models at the belly

The natural heart valve is known to have the capacity to regenerate, and Deck et al. [73] showed how the natural valve tends to produce collagenous protein in response to high stresses. Collagen fibers in the natural valve are oriented in the circumferential direction, so one can conclude that the predominating stresses in the circumferential direction cause the fiber architecture, further proving the importance of the circumferential properties of the valve. The radial characteristics of the valve allow valve coaptation during diastole and valve recoil during systole, but it can be assumed that just as long as the leaflet radial properties do not prevent valve closure or opening, they are not as critical to valve function. It was shown in these models that a large degree of change in the radial modulus had limited impact on the leaflet stress concentrations. For 
the circumferentially oriented JSI-reinforced leaflet mounted on the high profile stent (Figure 80) and the isotropic low modulus leaflet mounted on the high profile stent (Figure 95) the circumferential moduli were identical $(\mathrm{E}=2.97 \mathrm{MPa})$, but their radial moduli differed by $11.53 \mathrm{MPa}$; nevertheless, minimal impact to the leaflet stress concentration occurred. For the circumferentially oriented JSI leaflet, the radial modulus was higher than the circumferential modulus. In the natural valve (Figure 96), the radial modulus is approximately three times less than that of the circumferential modulus [73], and yet no significant change in the leaflet stress concentrations was witnessed. Based on these results, one can conclude that changing the radial modulus from six times as small to twice as large as the circumferential modulus has limited effect on the leaflet stress concentrations as long as the circumferential modulus is in the vicinity of that of the natural valve.

\subsection{Fatigue Model}

All previous experimentation ruled out all but the BARD PET knit as a feasible material for leaflet reinforcement. As a result, tension-tension fatigue was only carried out on these specific samples. Samples were manufactured by solvent casting, and S/N curves were generated for the circumferential direction, radial direction, and $45^{\circ}$ in between (Figure 101). If a sample reached 350 million cycles, the test was stopped, and the load was assumed to be below the endurance limit for the material. One such case occurred for testing in the $45^{\circ}$ direction, and it is represented by an open triangle in Figure 101. Each $\mathrm{S} / \mathrm{N}$ curve was fit to a $\log$-linear equation, represented in the figure below, and this was used for input into the fatigue prediction model. 


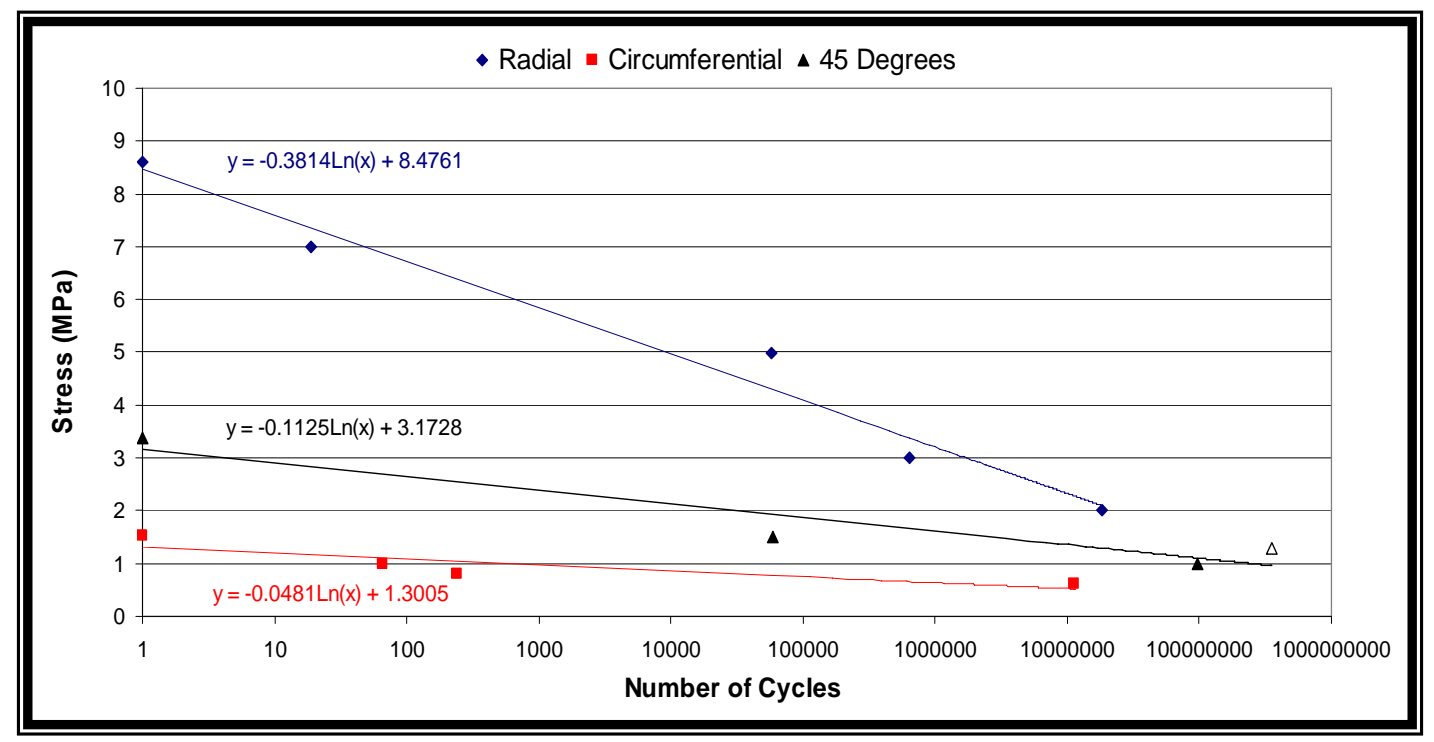

Figure 101. Cyclic tension-tension fatigue results for solvent-cast BARD reinforced leaflet samples along the circumferential, radial, and $45^{\circ}$ between the circumferential and radial directions. Each data set was fitted to a log-linear curve whose equation is provided in the figure.

The $\mathrm{S} / \mathrm{N}$ equations were used to predict the fatigue stress after $1-400$ million cycles when a sample was subjected to any of the three separate loading conditions used for tensile fatigue testing (Table 17). The radial, circumferential, and $45^{\circ}$ components were broken into their $\sigma_{11}$ and $\sigma_{22}$ components (refer to Figure 102) corresponding to the 1 and 2 directions in the finite element model. From this information, the $\sigma_{11}$ versus $\sigma_{22}$ stress interactions could be plotted, and a failure envelope could be defined by the three distinct points that were generated. Figure 103 will be used to describe the process of defining failure envelopes for specific lifetimes. Firstly, the coordinates of the three points defined for a specific lifetime (in this example, 1 million cycles), were plotted. A curve was then fit to this data to represent the interaction between the stresses in the 1 (circumferential) and 2 (radial) directions with respect to their impact on fatigue life. A curve linking all three points represents an equivalent fatigue life for all points along that 
curve. A quadratic equation was found to be sufficient to describe the failure curve for all cases represented in Table 17, where

Equation 14. General failure curve equation.

$$
\sigma_{11}(\text { model })=B \cdot\left(\sigma_{22}\right)^{2}+C \cdot\left(\sigma_{22}\right)+D
$$

In this case, the constants $(\mathrm{B}, \mathrm{C}$, and $\mathrm{D})$ are specific to each failure curve, and the solver tool in Excel was used to define these three constants for each distinct case represented in Table 17 by minimizing the sum squared error between the model and experimentally predicted values. Once all curves were generated, the stress components predicted during finite element modeling could be input to assess what fatigue range could be expected.

Table 17. Predicted failure stresses in the circumferential and radial directions for specific cyclic intervals from 1 to 400 million cycles.

\begin{tabular}{|c|c|c|c|c|c|}
\hline \multirow{2}{*}{$\begin{array}{l}\text { \# Cycles } \\
\text { (N) }\end{array}$} & \multirow{2}{*}{$\begin{aligned} \sigma_{11}= & -0.0481 \ln (\mathrm{N}) \\
& +1.3005\end{aligned}$} & \multirow{2}{*}{$\begin{aligned} \sigma_{22}= & -0.3814 \ln (N) \\
& +8.4761\end{aligned}$} & \multicolumn{3}{|c|}{$\sigma_{45^{\circ}}=-0.1125 \ln (N)+3.1728$} \\
\hline & & & $\sigma_{45^{\circ}}$ & $\begin{array}{c}\sigma_{11}= \\
\sigma_{45^{\circ}}\left(\sin 45^{\circ}\right) \\
\end{array}$ & $\begin{array}{c}\sigma_{22}= \\
\sigma_{45^{\circ}}\left(\sin 45^{\circ}\right) \\
\end{array}$ \\
\hline $1.00 \mathrm{E}+00$ & 1.300 & 8.476 & 3.173 & 2.244 & 2.244 \\
\hline $1.00 \mathrm{E}+02$ & 1.079 & 6.720 & 2.655 & 1.877 & 1.877 \\
\hline $1.00 \mathrm{E}+03$ & 0.968 & 5.841 & 2.396 & 1.694 & 1.694 \\
\hline $1.00 \mathrm{E}+04$ & 0.857 & 4.963 & 2.137 & 1.511 & 1.511 \\
\hline $1.00 \mathrm{E}+05$ & 0.746 & 4.085 & 1.878 & 1.328 & 1.328 \\
\hline $1.00 \mathrm{E}+06$ & 0.636 & 3.207 & 1.619 & 1.144 & 1.144 \\
\hline $1.00 \mathrm{E}+07$ & 0.525 & 2.329 & 1.360 & 0.961 & 0.961 \\
\hline $1.00 \mathrm{E}+08$ & 0.414 & 1.450 & 1.100 & 0.778 & 0.778 \\
\hline $4.00 \mathrm{E}+08$ & 0.347 & 0.922 & 0.945 & 0.668 & 0.668 \\
\hline
\end{tabular}

The same four regions used to compare the overall stress concentrations in the leaflet were used to determine the potential fatigue life of a BARD-reinforced valve. When the circumferential and radial stress components are represented for the whole leaflet (Figure 104), it is apparent that elevated stress areas exist at the top of the 
commissure, lower commissure, and center of coaptation for the circumferential component, and elevated stresses occur in the belly region and lower coaptation for the radial component. The numerical values of the peak stresses in the four critical regions at $0.0298,0.0571$, and 0.090 seconds were plotted together with the failure curves for assessment of fatigue life (Figure 105). It is apparent that the results for the lower commissure and belly region fall well within the 400 million cycle failure envelope. The peak load occurring at 0.090 seconds for the center of coaptation falls just inside the 400 million cycle failure envelope, but the 0.0571 and 0.090 second results for the top of the commissure both fall outside the 400 million failure envelope. As a result, one can conclude that the top of the commissure is the weak point during valve fatigue, and with the present design, failure can be expected before 400 million cycles. During valve fatigue testing, this particular design did fail at the top of the commissure (refer to Figure 64) at an equivalent of 4.19 years (approximately 168 million cycles). The valve happened to fail at a heat sealed joint, which is a well-known weak point on the valve that was not taken into account in the finite element model. As a result, the anticipated fatigue life for the BARD valve is closer to 400 million cycles (10 years) if a design change can effectively strengthen the weak joint in the leaflet.

According to Deck et al. [73] and Thubrikar et al. [74], the primary location of elevated stresses in both the natural valve and bioprosthetic valves occurs at the leaflet attachment zone, corresponding to the top of the commissure in the SIBS valve. Upon evaluation of the stresses and strains in the natural valve during both systole and diastole, Deck et al. [73] found that total stresses were elevated during diastole, and they were negligible during systole; therefore, the diastolic loading of the leaflet resulted in high 
tensile stresses, especially in the commissural region, where these high stresses are proposed to result in wear of the tissue that can ultimately lead to the valve's failure.

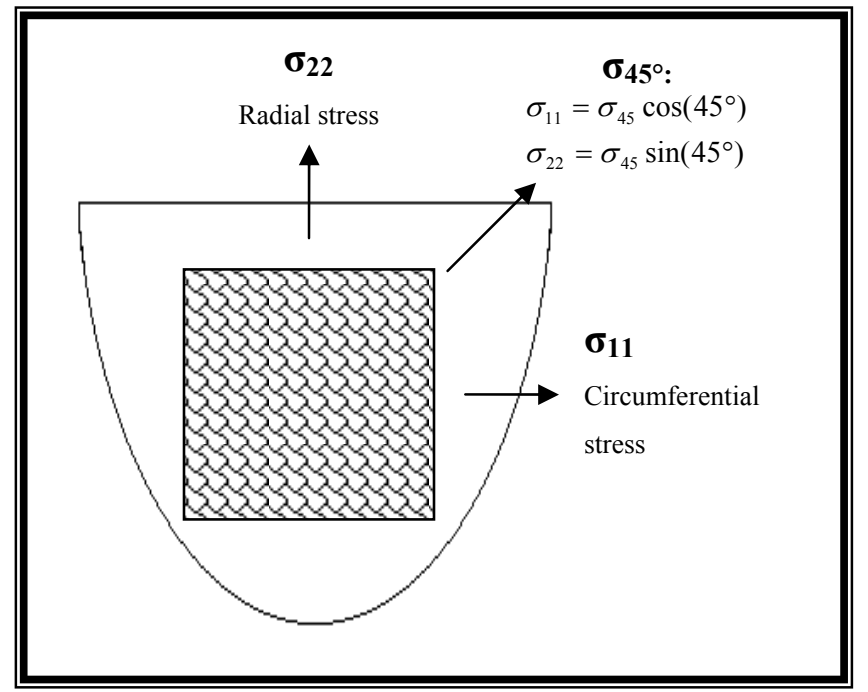

Figure 102. Representation of the principal directions subjected to tension-tension fatigue. The stresses along the $45^{\circ}$ orientation were broken up into their circumferential and radial components.

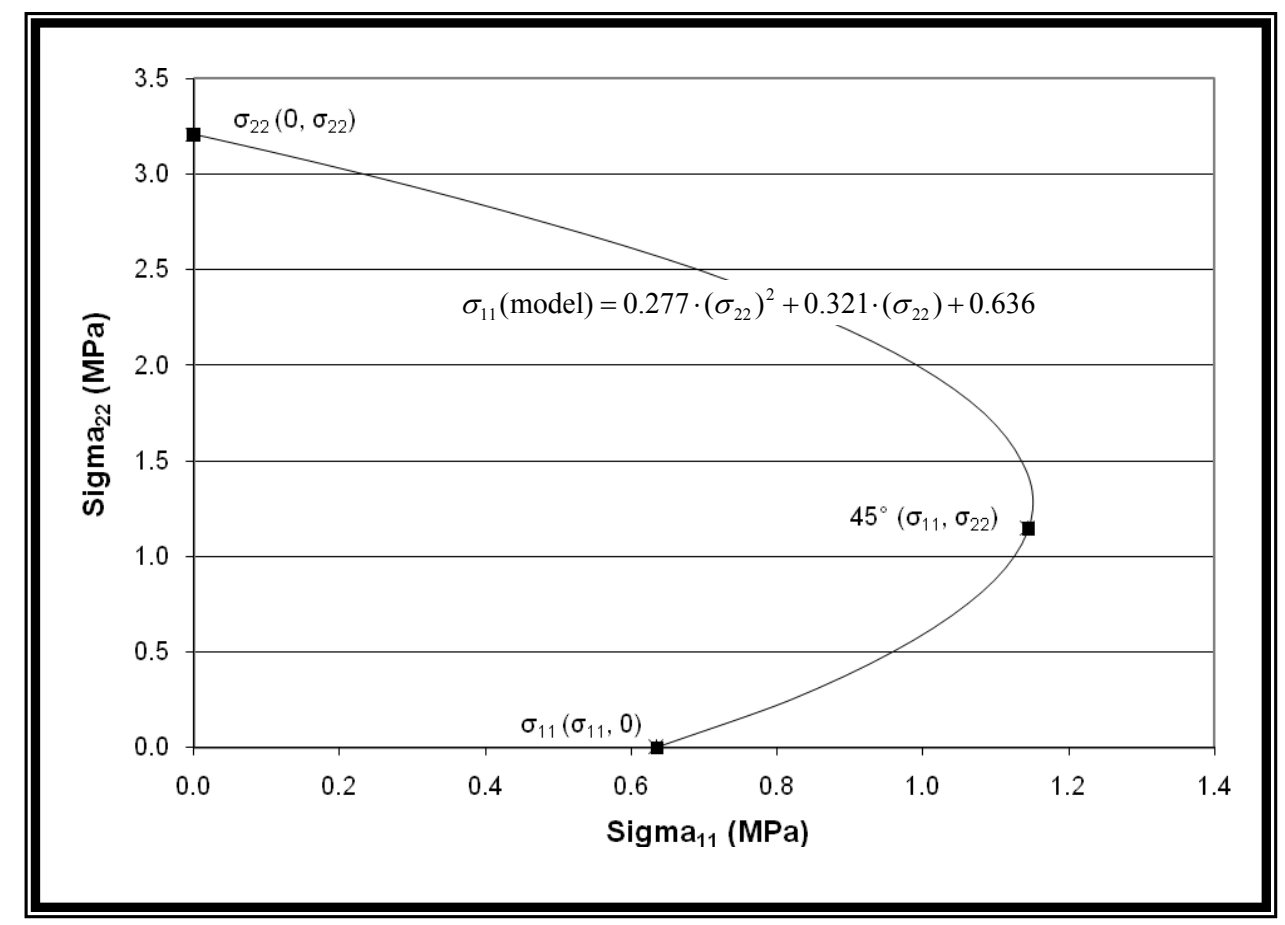

Figure 103. Representation of the method used to define a failure envelope for leaflet fatigue at 1 million cycles. 


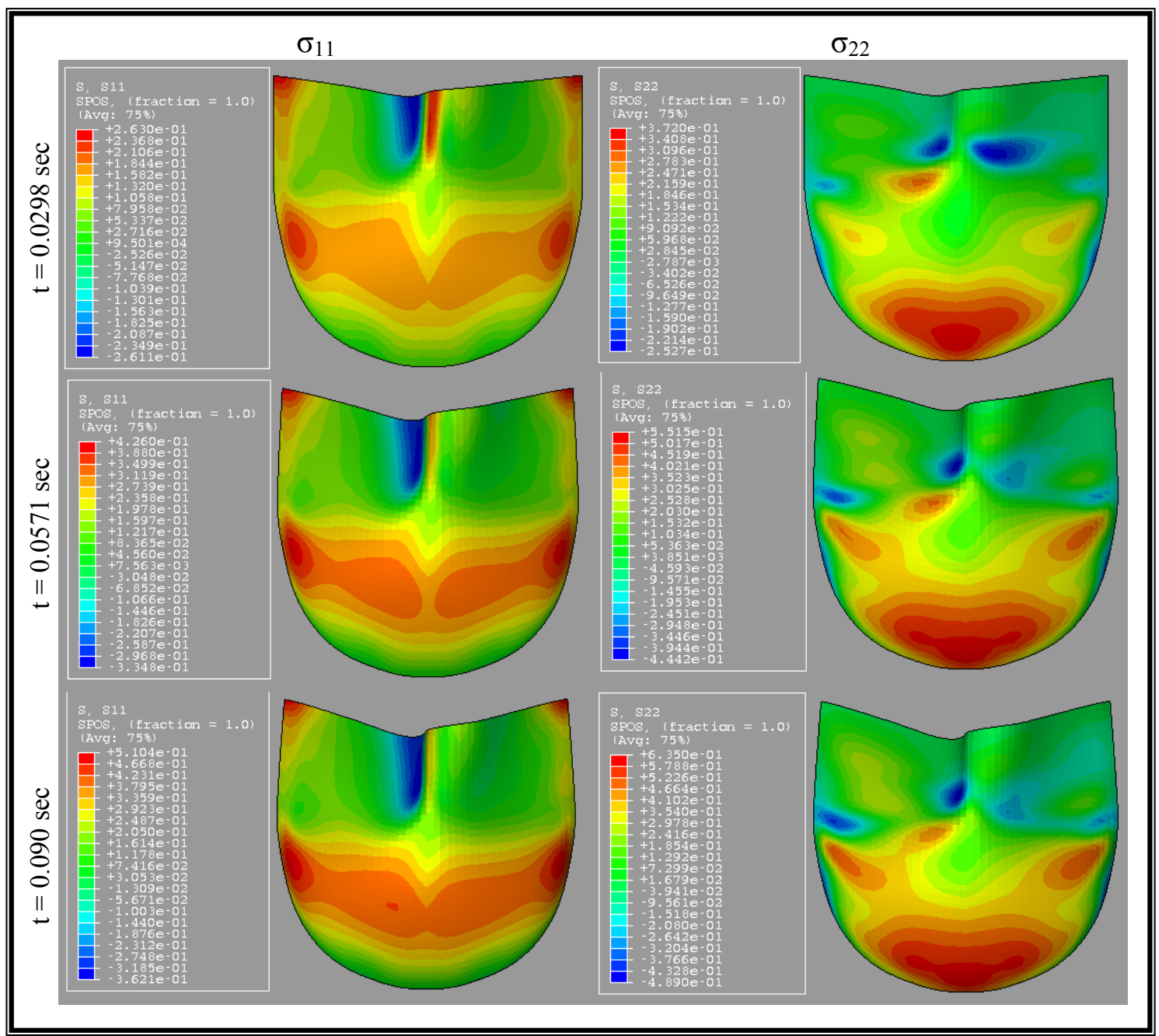

Figure 104. BARD-reinforced leaflet model mounted on a $48.5 \%$ styrene, medium profile stent. Images show the circumferential $\left(\sigma_{11}\right)$ and radial $\left(\sigma_{11}\right)$ stress components at 0.0298 seconds, 0.0571 seconds, and 0.090 seconds during the quasi-static diastolic loading. Note: stress shown in MPa.

The stresses occurring within the leaflet during diastole are as a result of the transvalvular pressure load that the leaflet is being subjected to. The leaflet deforms under the tensile load; therefore load controlled tensile fatigue testing is justified for fatigue analysis of polymeric leaflets. The affect of creep, on the other hand, was not taken into account as load controlled fatigue testing subjected a leaflet sample to a constant load until failure (50\% strain) occurred, and the $\mathrm{S} / \mathrm{N}$ curve results were reported as engineering stress versus number of cycles. According to Deck et al. [73], a reduction 
in the leaflet thickness causes a decrease in the bending strain and stress in a leaflet; however, an increase in the membrane or tensile stress occurs. The pressure load during diastole results in a decrease in the leaflet thickness due to an increase in its length [75]. Continual cycling of the leaflet will lead to a progressive decrease in its thickness due to creep. This decrease in thickness will cause an increase in stress, so failure of the leaflet can potentially occur before the predicted 400 million cycles. Due to this, it is recommended that a factor of safety (FOS) be applied to ensure that catastrophic valve failure does not occur before the valve's predicted lifespan.

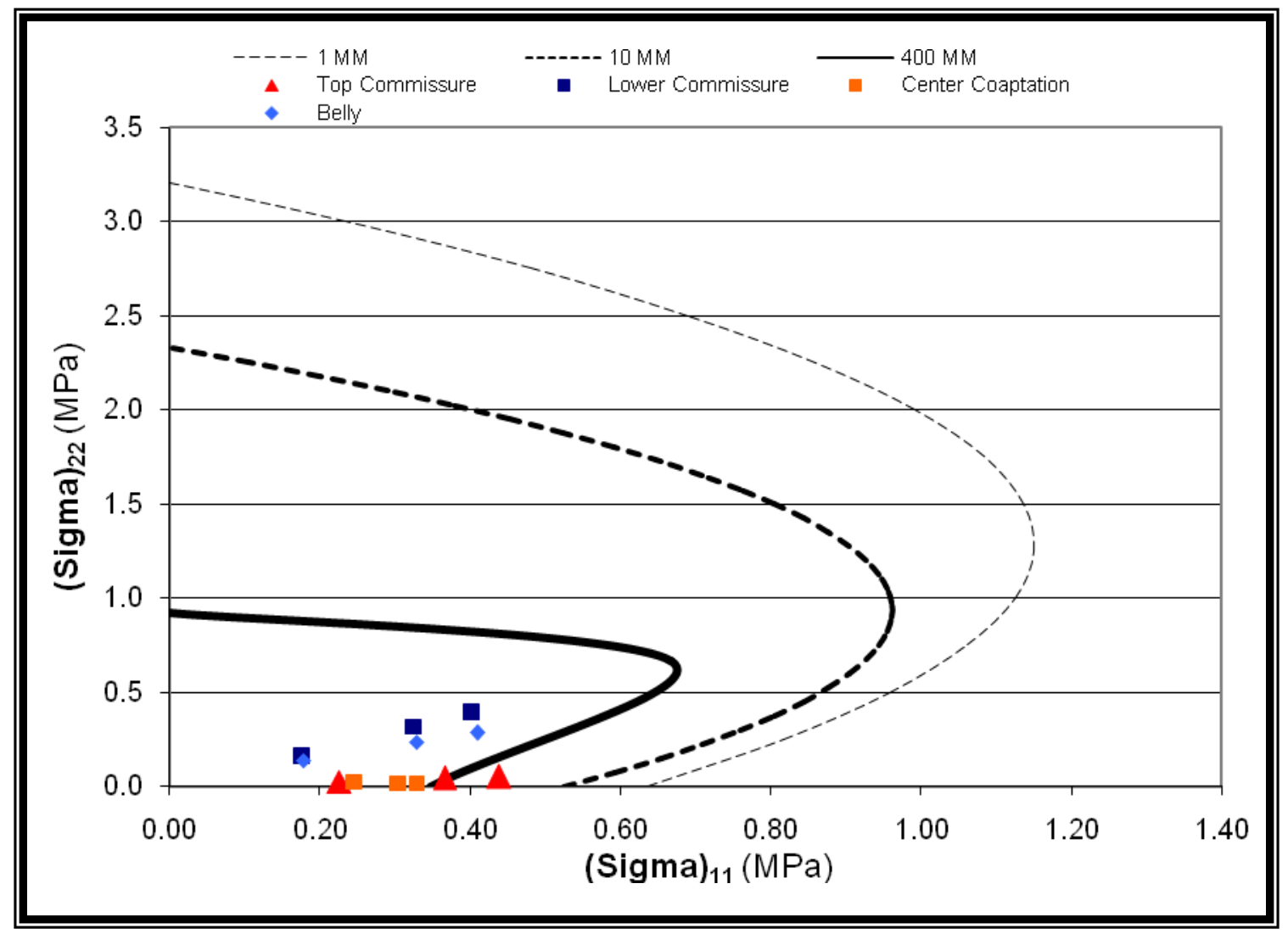

Figure 105. Failure envelopes predicted for the BARD-reinforced leaflets, showing the 1 million, 10 million, and 350 million cycle failure envelope. The model-predicted stress components for the top of the commissure, the lower commissure, the center of coaptation, and the belly region are plotted for 0.0298 seconds, 0.0571 seconds, and 0.090 seconds for assessment of fatigue life. 
The natural valve is endowed with the capacity to regenerate. Studies performed by Deck et al. [73] showed an increase in glycosaminoglycan and collagenous protein synthesis in a rat valve when compared with other organs within its body. This elevated rate of tissue replacement was proposed to be as a result of the high stresses, thereby allowing the valve to maintain its function without degeneration. In bioprosthetic valves, regeneration of the collagenous network obviously cannot occur, and breakdown of the collagenous fibers has been noted as one of the failure modes [73]. Another interesting point to note is the presence of glycosaminoglycans: glycosaminoglycans are believed to contribute to a reduction in the stresses within the leaflet as these molecules allow slippage between the fibrous elements, thereby increasing the durability of the leaflets. Glutaraldehyde fixation of bioprosthetic valves has been linked to a reduction in the presence of glycosaminoglycans. Considering the rapid production of these molecules in the natural valve, they obviously play an important function in the mechanics and longevity of the natural valve. The lack of this friction-reducing molecule in both bioprosthetic valves and polymeric valves put them at a disadvantage in terms of fatigue life when compared with the natural valve; nevertheless, implementing materials and geometries that can minimize the stresses in a valve can have a marked impact on their longevity. 


\section{SUMMARY AND CONCLUSIONS}

Polymer trileaflet heart valves have been under investigation since the 1960's, but they have met with limited success due to poor material and design choices [4-6, 22-30]. Many materials investigated for use in leaflets were not biostable, and chemical degradation of these materials, in combination with mechanical fatigue, accelerated their failure. Leaflets are subjected to alternating tension and compression during the cardiac cycle; these alternating stresses result in the mechanical degradation of the leaflet materials and the formation of cracks and tears, ultimately leading to the catastrophic failure of the valve. Designs that aid in the reduction of stress concentrations in the leaflet are proposed to increase the valve's overall durability [29]. Design aspects that are believed to reduce leaflet stress concentrations include stent flexibility, parabolic coaptation curvature, and leaflet anisotropy [28-30, 37, 38, 41, 44, 49, 51, 52]. It is with these concepts in mind that a fiber-reinforced SIBS trileaflet valve was developed.

SIBS is a triblock copolymer that has been proven to be stable in oxidative environments [8]. Being a block copolymer, its hard and soft segment chemistry can be manipulated to produce polymers with a large variety of mechanical properties. By increasing the styrene percentage in the polymer, the stiffness of the material can be increased. This property of the polymer was exploited in the valve, where a low percentage styrene SIBS was used for the leaflet matrix, and a high percentage styrene SIBS was used for the supporting stent. Prior studies have proven the feasibility of a SIBS trileaflet valve in terms of its biocompatibility and acute function [7, 9-14], and it was the intention of this investigation to refine the design in order to optimize valve durability while improving the hydrodynamic function. 
An extensive literature review of the natural heart valve and bioprosthetic valves revealed that it is not necessary to replicate all of the properties of the natural valve in order to achieve sufficient durability [21, 31-36, 39, 40, 49, 51-53, 58, 60, 62-65]. One of the most critical features of the natural valve is its collagen network. The layout of the collagen network throughout the leaflet allows the valve to withstand high tensile loads while not preventing extensibility and bending. Radial extensibility of leaflets is important during diastole as it promotes leaflet coaptation and reduces regurgitation. Low resistance to bending allows the leaflets to open freely during systole, thereby allowing favorably low pressure drops. The collagen network was simulated in the SIBS valve through the incorporation of a commercially available reinforcement fabric. The exact mechanical properties of the natural valve could not be matched, so a combination of experimentation and modeling was used to evaluate what reinforcement type and orientation provided the best possible combination of acute and long-term valve function. Seven different polyester reinforcement materials were tested using four different processing methods to maximize the material property combinations.

The flexibility of the supporting stent is also believed to have a large impact on valve function and durability $[28,30,41,52]$. In the natural valve, the leaflets join the aortic wall at the commissures, and the aortic wall dilation and contraction has been linked to improved hemodynamic function and reduced stress load in the area. During systole, the aortic wall dilates, pulling the commissures apart and aiding in leaflet opening. During diastole, the inward flexion of the commissure helps reduce stress concentrations in that area. A combination of three stent geometries and two SIBS grades were used during the experimental evaluation, and a combination of two stent 
geometries and three different material properties were used during finite element modeling to verify the importance of stent flexibility.

The coaptation geometry is believed to play an important role in commissure stress concentrations and regurgitation. A more parabolic coaptation geometry is believed to reduce the load on the leaflet, but a leaflet that does not have sufficient radial extensibility will not close completely if it has a parabolic coaptation geometry. For example, pericardial valves have reduced radial extensibility due to glutaraldehyde fixation, and these valves require a flat coaptation geometry in combination with a spherical leaflet shape in order to achieve valve closure. The geometry of the pericardial valve has proven to result in increased stress concentrations when compared with the natural and porcine valve leaflets [21]. In order to verify this phenomenon for the SIBS valve, three different coaptation geometries were evaluated by finite element modeling.

The two primary experimental methods used to evaluate the feasibility of the composite leaflet materials included valve hydrodynamic testing and durability testing. The preliminary valve design included three individually placed spherical leaflets, but this valve failed prematurely due to leaflet-stent detachment and polymer degradation. The valve incorporated an open PET knit which allowed exposed polymer. The individually placed spherical leaflets were replaced by a continuous cylinder of leaflet material that was sutured to the supporting stent. The curved leaflet geometry was obtained by thermal-forming techniques, and this new design eliminated the problem of leaflet-stent detachment. Upon fatigue testing, it was immediately obvious that a dense reinforcement fabric was required to sufficiently support the SIBS matrix, as the PET mesh reinforcement that had large enough interstices to see visibly unsupported matrix 
material failed prematurely (after 0.908 years) due to polymer blowout from between the interstices.

The first cylinder valve design incorporated a high profile, $30 \%$ styrene stent, resulting in increased flexibility when compared with the original three leaflet, low profile valve. In vitro testing showed improved hydrodynamic function and significantly increased durability (failure after 10.35 years due to system-related problems), but when this valve was implanted in the aortic position of an ovine model, severe stent distortion rendered the valve incompetent. It was concluded that the increased flexibility and design changes were beneficial to the in vitro function; however, the stent was now too flexible for in vivo use. In addition, an inflammatory response was seen in vivo, and it was concluded to be as a result of exposed Dacron fibers. Dacron is a well-known cause of inflammation [71]. As a result, the stent design was changed to a medium profile, $48.5 \%$ styrene SIBS formulation, and the processing technique used for the leaflets was changed to improve coating. The hydrodynamic function of this valve once again improved, but failure in vivo due to an inflammatory response was still a problem. SEM analysis of a fatigued valve sample showed that the exposure of Dacron fibers persisted with this new leaflet manufacturing technique. The nominal thickness of the uncoated Dacron used in these cases was $0.30 \mathrm{~mm}$, but in order to achieve preferential hydrodynamic function, a thin leaflet was required. By attempting to maintain as thin a leaflet as possible, insufficient polymer coating was applied to the underlying Dacron. From this, it was concluded that a significantly thinner fiber reinforcement was necessary in order to obtain complete coverage while not creating a thick, stiff leaflet that is resistant to bending. 
In vitro and in vivo testing found no fault with the $48.5 \%$ styrene, medium profile stent, so this design was continued with the application of varying polymer reinforcements. Two new mesh designs, one weave, and one new knit design were evaluated for use as leaflet reinforcement. Each had a nominal thickness of approximately $0.15 \mathrm{~mm}$, and a solvent casting technique was instituted to ensure complete coverage. The coated thickness of these materials was approximately $0.20 \mathrm{~mm}$. Hydrodynamic testing of these materials showed a decreased pressure drop when compared with the original designs, and variable regurgitation. The valve design with the highest regurgitation (the NZ-11 mesh-reinforced design) coincidentally had the highest modulus in both the circumferential and radial directions, but upon comparison of the modulus versus both percent regurgitation and pressure drop, no distinct correlation between these properties was found. There was, however, a correlation between leaflet thickness and transvalvular pressure drop, where thinner leaflets generally resulted in lower pressure drops.

Due to the fact that the Dacron-reinforced valve with the highly flexible stent posts showed the best results in terms of durability, a valve including Dacron-reinforced leaflets mounted on the medium profile, $48.5 \%$ styrene stent was manufactured for comparison. Fatigue analysis of the two new mesh-reinforced valves (NZ-11 and XA47), the weave-reinforced valve (fibers rotated $45^{\circ}$ from the circumferential direction), and the two PET knit/Dacron-reinforced valves (JSI (original) and BARD) revealed that the two Dacron-reinforced valves had the most promising fatigue results. Upon analysis of the modulus of these two knits, neither the properties in the circumferential nor radial directions were found to be significantly different, so the only difference was their 
thickness. The JSI-reinforced leaflet had an overall thickness of $0.40 \mathrm{~mm}$, and the BARD-reinforced leaflet had a thickness of $0.20 \mathrm{~mm}$. Hydrodynamic comparison of these two valves showed a $28.9 \mathrm{mmHg}$ difference in mean transvalvular pressure drop, providing a stronger argument for the importance of thin leaflets when considering a valve's hydrodynamic function. Fatigue failure of the BARD valve at an equivalent of 4.19 years was as a result of the heat-seal degradation and not actual reinforcement failure. The cylinder valves were all manufactured from flat sheets, and they were formed into a cylinder by heat-sealing the edges. This particular joint has proven to be a weak point in the valve design, and future work involves the replacement of the heat-seal with a reinforced suture line to provide a stronger attachment point. The actual BARDreinforced fabric showed no signs of degradation, so the improvement of the leaflet seal is hypothesized to significantly increase its durability. An analysis of the leaflet modulus versus fatigue life once again showed no correlation between the two properties.

The premature fatigue of all but the knit-reinforced samples prompted the finite element analysis of only these two reinforcement types. A total of eleven different models were run, which investigated the impact of stent flexibility and geometry, leaflet thickness and anisotropy, and coaptation geometry on leaflet stress concentrations. A uniform transvalvular pressure load was enforced on the aortic surface of the leaflets, and the amplitude was varied according to the loading curve obtained during hydrodynamic testing of the BARD-reinforced valve (slight coaptation curvature and $48.5 \%$ styrene stent). It was only possible to establish the effect of diastolic loading during quasi-static analysis as numerical instabilities resulted in premature termination of all static analyses of systolic valvular loading. Diastolic loading was believed to provide sufficient insight 
as to the effects of the different parameters on the leaflet stress concentration as Deck et al. [73] reported that stresses during systole were negligible, and diastolic stresses were critical for the understanding of natural valve leaflet remodeling and fatigue.

The four primary areas in the valve that were of concern in terms of elevated stress concentrations included the top of the commissures, the lower commissure, the center of coaptation, and the belly region. Leaflet stress concentrations on both the aortic and ventricular surfaces were computed, and for the fiber reinforced valves, the lower commissure was the only region that displayed a large difference between the stress concentrations on the aortic and ventricular surfaces, where the aortic surface consistently showed higher stress levels than the ventricular surface.

Increasing stent flexibility from completely rigid to marginally flexible (385 $\mathrm{MPa}$ ) for the BARD-reinforced valve resulted in a significant decrease in the peak stress concentrations at the top of the commissure, which is consistent with reports in the literature [21]. A further decrease in stent stiffness (to $68 \mathrm{MPa}$ ), however, did not present any additional benefit to the stress concentration. For the JSI-reinforced valve, the stress concentrations were compared between the medium profile, $48.5 \%$ stent and the high profile, $30 \%$ stent. No significant difference between stress concentrations was established, so it can be concluded that reducing stent rigidity does initially lead to a reduction in the stress concentration at the top of the commissure, but the rate of improvement of stress concentration plateaus with a further reduction of stent modulus. From this, it can be concluded that a balance needs to be found where the stent should be flexible enough to help reduce high stress concentrations, but it should not be so flexible that it cannot maintain its integrity in vivo. 
The two experimental JSI valves mounted on the high profile, $30 \%$ styrene stent were evaluated to determine the affect of varying reinforcement orientation. Fatigue analysis proved the valve with the radially oriented maximum compliance to have a significantly lower fatigue life (4.42 years) compared with the circumferentially oriented leaflets (10.35 years). Finite element modeling of these two designs revealed increased stress concentration in the region of the lower commissure, center of coaptation, and the mid valve for the radially oriented leaflets. The peak stress location on the radially oriented leaflets coincided with a failure point seen during dynamic fatigue, further corroborating the relationship between stress concentrations and durability. The natural heart valve has increased compliance in the radial direction when compared with the circumferential direction, but the actual modulus is markedly lower than any of the materials incorporated for use in the synthetic valve. As a result, maintaining the circumferential compliance is believed to be more important in terms of reduced stress concentrations and increased valve durability than mimicking the natural valve trends. In order to further verify this statement, three additional models of the high profile, $30 \%$ styrene stent were evaluated incorporating either a low $(E=2.97 \mathrm{MPa})$ or high $(E=14.5$ MPa) modulus isotropic leaflet or an orthotropic leaflet mimicking the properties of the natural valve $\left(\mathrm{E}_{\text {circ }}=5.79 \mathrm{MPa}, \mathrm{E}_{\mathrm{rad}}=0.966 \mathrm{MPa}\right) . \quad$ The low and high modulus properties were obtained from the two orthotropic directions of the dip-coated JSI Dacron. What was found was that the high modulus isotropic valve replicated the elevated stress concentrations of the radially oriented JSI-reinforced valve, and the low modulus and natural valve model replicated the more optimal results from the circumferentially oriented valve. Thubrikar et al. [75] believed that the valve leaflets are 
subjected to flexural and tensile stresses predominantly in the circumferential direction, and when the stress concentrations for all eleven models were divided into their circumferential and radial components, it was evident that the circumferential stress levels were consistently higher than the radial. Based on these results, it was concluded that matching the circumferential modulus of the natural valve was more important in terms of the reduction in leaflet stresses than mimicking the radial versus circumferential modulus trends. Moreover, the results proved that changing the radial modulus from six times as small to twice as large as the circumferential modulus had limited impact on the leaflet stress concentrations as long as the circumferential modulus was in the vicinity of that of the natural valve.

A literature review of the effect of coaptation geometry has consistently revealed that a parabolic geometry is better than a flat geometry for reduced leaflet stress concentrations $[21,49,51]$. Three models of the BARD valve incorporating three different coaptation geometries showed no change in the leaflet stress concentrations, so it was concluded that the choice of coaptation geometry should be dependent on valve regurgitation. Too large a coaptation curvature can prevent complete valve closure, leading to regurgitation.

It was interesting to note that increasing leaflet thickness resulted in an overall decrease in leaflet stress concentrations. Models of BARD- and JSI-reinforced valves mounted on a $48.5 \%$ styrene stent were compared, and the thicker, JSI leaflet led to a consistently lower leaflet stress concentration during diastolic loading. According to Deck et al. [73], an increase in the leaflet thickness causes an increase in bending strain and stress in a leaflet and a decrease in tensile stress. What this means is that, although 
the leaflet stress is reduced, the increased bending stress and strain results in an increase in the transvalvular pressure drop during hydrodynamic testing of the valve, as was seen for the two valve types.

From the combination of experimental and model evaluations, it can therefore be concluded that the BARD-reinforced leaflet with medium coaptation curvature and $48.5 \%$, medium profile stents presents the most promising combination of results. Although the thicker, JSI reinforcement has a proven durability and a reduction in stress concentrations during modeled diastolic loading, in vivo evaluation ruled it out as a potential candidate. The thinner BARD-reinforced leaflets showed excellent hemodynamics when compared with the Magna bioprosthesis and other SIBS valve prototypes. Due to this, the BARD-reinforced valve was selected for analysis of fatigue life using a method reminiscent of that developed by Hashin [67 - 70].

An empirical fatigue failure criterion was developed to represent failure curves at select cyclic intervals. BARD-reinforced leaflet samples were subjected to dynamic tensile fatigue along their circumferential direction, radial direction, and $45^{\circ}$ in between. The $\mathrm{S} / \mathrm{N}$ curves that were generated were fit to log-linear equations, each representing the characteristic mean stress versus number of cycles to failure for each of the three principal directions. The equations generated were used to predict the characteristic failure stress for specific cyclic intervals ranging from $1-400$ million cycles, and these stress components were broken into their characteristic circumferential and radial components. A plot of the circumferential versus radial failure stress components was made, and an independent failure curve was defined for each set of three points corresponding to one lifespan. A quadratic approximation was found to be the most 
appropriate fitting curve for the three experimental points. Once the fatigue failure criteria had been established, the model-generated results for the circumferential and radial components for the same four critical regions specified for stress concentrations were plotted on the generated figure. Each location output three separate coordinate pairs corresponding to $0.0298,0.0517$, and 0.090 seconds on the diastolic loading curve. Based on the location of the points in the fatigue limit region or envelope, the fatigue life of the valve was predicted. What was found was that the top of the commissure represented the critical region in predicting fatigue failure, similar to what has been witnessed in both the natural valve and bioprosthetic valves [73 - 75]. Based on the maximum diastolic loading conditions, the valve is predicted to fail prior to 400 million cycles (10 years). Experimental fatigue of this valve revealed that failure did occur at the top of the commissure at an equivalent of 4.19 years ( 167 million cycles), but failure was as a result of degradation of the heat-sealed joint, a design specification not taken into account during modeling. The fatigue prediction model is in its very early stages, so additional experimental validation is required to refine the characteristics contributing to leaflet failure. In this model, the shear stress and shell normal stress were neglected.

DCSP was applied for validation of the finite element model in this research. It proved to be unsuccessful for the accurate prediction of leaflet deformation during the cardiac cycle; nevertheless, the methodology and results were presented in this discussion because the method is still believed to be a potentially useful one for model validation. Issues relating to the experimental set-up reduced measurement accuracy, but if these problems can be corrected, it could prove to be a valuable tool. 
In order to conclusively prove the potential of the BARD-reinforced leaflet mounted on the $48.5 \%$ styrene stent, continued in vitro testing is needed to determine the best coaptation geometry for improved hydrodynamics, and the long-term fatigue of the valve needs investigation through a combination of in vitro fatigue and in vivo implantation. Concern as to the lack of fatigue resistance at the top of the commissure may warrant further reinforcement in that area, with the understanding that reinforcement should not inhibit bending in this critical region.

In reference to the questions from the specific aims, the best possible valve design for the SIBS trileaflet valve incorporates the Design 4 valve including the BARDreinforced leaflet and medium profile, $48.5 \%$ styrene stent. A limited amount of stent flexibility does lead to a reduction in leaflet stress concentrations; however, excessive flexibility has no added benefit. Exact replication of the natural heart valve properties is not necessary when designing a prosthetic heart valve; however, the circumferential properties of the leaflet have proven to be more important than the radial properties. Replication of the circumferential properties of the natural heart valve can produce a prosthetic valve with reduced leaflet stress concentrations and increased durability, while a large variation in radial modulus has minimal effect. Increased leaflet thickness results in a decrease in leaflet stress concentrations but an increase in transvalvular pressure drop; therefore leaflet design is required to optimize these two contradicting characteristics. A simplified fatigue prediction model, such as that produced herein, can be used to identify the weak points in the valve design; however, additional investigation is required to evaluate the accuracy of the model. 


\section{REFERENCES}

1. American Heart Association. Heart and Stroke Statistical Update. 2006, American Heart Association: Dallas, TX.

2. Sako EY. Newer concepts in the surgical treatment of valvular heart disease. Current Cardiology Reports 2004; 6: 100-105.

3. Morsi YS, Birchall IE, Rosenfeldt FL. Artificial aortic valves: an overview. The International Journal of Artificial Organs 2004; 6: 445-451.

4. Daebritz SH, Fausten B, Hermanns B, Schroeder J, Groetzner J, Autschbach R, Messmer BJ, Sachweh J. Introduction of a polymeric heart valves prosthesis with special design for the aortic position. European Journal of Cardio-thoracic Surgery 2004; 25: 946-952.

5. Imamura E, Kaye MP. Function of expanded polytetrafluoroethylene laminated trileaflet valves in animals. Mayo Clinic Proceedings 1977; 52: 770-775.

6. Hyde JAJ, Chinn JA, Phillips RE. Polymer heart valves. Journal of Heart Valve Disease 1999; 8: 331-339.

7. Reul H. In-vitro evaluation of artificial aortic valves. Adv Cardiol Phy 1983; 5: 1630 .

8. Gallocher SL, Aguirre AF, Kasyanov V, Pinchuk L, Schoephoerster RT. A novel polymer for potential use in a trileaflet heart valve. Journal of Biomedical Materials Research B: Applied Biomaterials 2006; 79: 325-334.

9. Pinchuk L, Khan IJ, Martin JB, Wilson GJ. Polyisobutylene-based thermoplastic elastomers for ultra long-term implant applications. Transactions of the Society of Biomaterials, Sixth World Biomaterials Congress Transactions 1999: 1452.

10. Schoephoerster RT, Gallocher S, Pinchuk L, and Kasyanov VA. A novel trileaflet synthetic heart valve. Proceedings of the 2001 ASME IMECE, New York, New York, November, 11-16, 2001.

11. Schoephoerster RT, Gallocher S, Aguirre A, Pinchuk L, and Kasyanov V. A Polymer Composite Trileaflet Aortic Heart Valve. Proceedings of the 6th Annual Hilton Head Workshop: Prosthetic Heart Valves, Hilton Head Island, SC, March 6-10, 2002. 
12. Wang Q, Mathison M, Gallocher S, Jaramillo F, Pinchuk L, Schoephoerster RT. Feasibility analysis of a stent-valve combination device: in vivo evaluation of the novel polymer valve and in vitro evaluation of an alternative fixation method. Proceedings of the 2005 BMES Annual Fall Meeting, Baltimore, MD, September 28 - October 1, 2005

13. Gallocher SL, Aguirre AF, Kasyanov V, Pinchuk L, Schoephoerster RT. Durability assessment of a novel polymer trileaflet heart valve. Proceedings of the First International Conference on Mechanics of Biomaterials \& Tissues, Waikoloa Beach Marriott Resort, Hawai'i, USA, 11 - 15 December 2005.

14. Schoephoerster RT, Gallocher S, Wang Q, Jaramillo F, Kato YP, Pinchuk L. Polymer valve with potential for minimally invasive and transcatheter delivery. Proceedings of the 10th Annual Hilton Head Workshop: Prosthetic Heart Valves, Hilton Head Island, SC, March 1-5, 2006.

15. Gallocher SL, Wang Q, Mathison M, Kato YP, Pinchuk L, Schoephoerster RT. Feasibility analysis of the safety and durability of a polymer trileaflet heart valve. Proceedings of the 2006 Summer Bioengineering Conference, Amelia Island, FL, June 21-25, 2006.

16. Tortora GJ, Grabowski SR. Principles of Anatomy and Physiology. New York: HarperCollins, 1996.

17. Bender JR. Yale University School of Medicine Heart Book: Heart Valve Diseases. Zaret, Moser, Cohen, ed. New York: Hearst Books, 1992.

18. Martini F. Fundamentals of Anatomy and Physiology. New Jersey: Prentice Hall, 2001.

19. Senthilnathan V, Treasure T, Grunkemeir G, Starr A. Heart valves: which is the best choice? Cardiovascular Surgery 1999; 7: 393-397.

20. DeWall RA, Qasim N, Carr L. Evolution of mechanical heart valves. Annals of Thoracic Surgery 2000; 69: 1612-1621.

21. U.S. opportunities in heart valve disease management. Health Research International 2002: \#053-1-US-0202.

22. Vesely I. The evolution of bioprosthetic heart valve design and its impact on durability. Cardiovascular Pathology 2003; 12: 277-286.

23. Mackay TG, Wheatley DJ, Bernacca GM, Fisher AC, Hindle CS. New polyurethane heart valve prosthesis: design, manufacture and evaluation. Biomaterials 1996; 17: $1857-1863$. 
24. Mackay TG, Bernacca GM, Fisher AC, Hindle CS, Wheatley DJ. In vitro function and durability assessment of a novel polyurethane heart valve prosthesis. Artificial Organs 1996; 20: 1017-1025.

25. Bernacca GM, Straub I, Wheatley DJ. Mechanical and morphological study of biostable polyurethane heart valve leaflets explanted from sheep. Journal of Biomedical Materials Research 2002; 61: 138-145.

26. Herold M, Lo HB, Reul H, Muckter H, Taguchi K, Giersiepen M, Birkle G, Hollweg G, Rau G, Messmer BJ. The Helmholtz Institute Trileaflet Polyurethane Heart Valve Prosthesis: Design, Manufacturing, and First In Vitro and In Vivo Results. In Polyurethanes in Biomedical Engineering II. (Eds Plank et al.) Elseveri 1987: 231256.

27. Daebritz SH, Sachwej JS, Hermanns B, Fausten B, Franke A, Groetzner J, Klosterhalfen B, Messmer BJ. Introduction of a flexible polymeric heart valve prosthesis with special design for the mitral position. Circulation 2003; 108: II134II139.

28. Pinchuk L. A review of the biostability and carcinogenicity of polyurethanes in medicine and the new generation of 'biostable' polyurethanes. Journal of Biomaterials Science Polymer Edition 1994; 6: 225-267.

29. Cacciola G, Peters GWM, Baaijens FPT. A synthetic fiber-reinforced stentless heart valve. Journal of Biomechanics 2000; 33: 653-658.

30. De Hart J, Cacciola G, Schreurs PJG, Peters GW. A three-dimensional analysis of a fibre-reinforced aortic valve prosthesis. Journal of Biomechanics 1998; 31: 629-638.

31. Cacciola G, Peters GWM, Schreurs PJG. A three-dimensional mechanical analysis of a stentless fiber-reinforced aortic valve prosthesis. Journal of Biomechanics 2000; 33: 521-530.

32. Doehring TC, Kahelin M, Vesely I. Mesostructures of the aortic valve. The Journal of Heart Valve Disease 2005; 14: 679-686.

33. Vesely I. The role of elastin in aortic valve mechanics. Journal of Biomechanics 1998; 31: 115-123.

34. Lovekamp JJ, Simionescu DT, Mercuri JJ, Zubiate B, Sacks MS, Vyavahare NR. Stability and functionality of glycosaminoglycans (GAGs) in porcine bioprosthetic valves. Biomaterials 2006; 27: 1507-1518.

35. Sacks MS, Smith DB, Hiester ED. The aortic valve microstructure: Effects of transvalvular pressure. Journal of Biomedical Materials Research 1998; 41: 131-141. 
36. Vesely I. The role of elastin in aortic valve mechanics. Journal of Biomechanics 1998; 31: 115-123.

37. Kasyanov VA, Purinya BA, Ose VP. Structure and mechanical properties of the human aortic valve. Mechanics of Composite Materials 1984; 5: 637-647.

38. De Hart. Fluid structure interaction in the aortic heart valve: A three-dimensional computational analysis. PhD. Thesis 2002. Technische Univerisiteit Eindhoven.

39. De Hart J, Peters GWM, Schreurs PJG, Baaijens FPT. Collagen fibers reduce stresses and stabilize motion of aortic valve leaflets during systole. Journal of Biomechanics 2004; 37: 303-311.

40. Mirnajafi A, Raymer J, Scott MJ, Sacks MS. The effects of collagen fiber orientation on the flexural properties of pericardial heterograft biomaterials. Biomaterials 2005; 26: 795-804.

41. Mirnajafi A, Raymer JM, McClure LR, Sacks MS. The flexural rigidity of the aortic valve leaflet in the commissural region. Journal of Biomechanics 2006; In publication.

42. Cacciola GR. Design, simulation and manufacturing of fiber reinforced polymer heart valves. PhD. Thesis 1998. Technische Univerisiteit Eindhoven.

43. Dowling NE. Mechanical Behavior of Materials: Engineering Methods for Deformation, Fracture, and Fatigue. New Jersey: Prentice Hall, 1999.

44. Gallocher SL. A novel polymer heart valve: quantification of hydrodynamic function and platelet deposition. Master's Thesis 2004. Florida International University: Department of Biomedical Engineering.

45. Liu Y, Kasyanov VA, Schoephoerster RT. Effect of fiber orientation on the stress distribution within a polymer composite heart valve in the closed position. Journal of Biomechanics 2007; 40: 1099-1106.

46. Lee B, Byrne J, Schoephoerster RT. Reconstruction of a trileaflet heart valve leaflet using photogrammetry and biquintic finite element method. Proceedings of the 2003 Summer Bioengineering Conference, Key Biscayne, FL, June 25-29.

47. Yin W, Gallocher S, Pinchuk L, Schoephoerster RT, Jesty J, Bluestein D. Flow induced platelet activation in a St. Jude mechanical heart valve, a trileaflet polymeric heart valve, and a St. Jude tissue valve. Artificial Organs 2004; 29: 829-831. 
48. Iwasaki K, Umezu M, Iijima K, Imachi K. Implications for the establishment of accelerated fatigue test protocols for prosthetic heart valves. Artificial Organs 2002; 26: 420-429.

49. Reul H, Potthast K. Durability / wear testing of heart valve substitutes. Journal of Heart Valve Disease 1998; 7: 151-157.

50. Chew GG, Howard IC, Patterson EA. Non-linear finite element modeling of porcine bioprosthetic valves. Engineering Failure Analysis 1994; 1: 231-242.

51. Ottosen N, Petersson H. Introduction to the Finite Element Method. Prentice Hall, Hertfordshire, 1992.

52. Christie GW. Computer modeling of bioprosthetic heart valves. European Journal of Cardiothoracic Surgery 1992; 6: S95-S101.

53. Li J, Luo XY, Kuang ZB. A nonlinear anisotropic model for porcine aortic heart valves. Journal of Biomechanics 2001; 34: 1279-1289.

54. Arcidianco G, Corvi A, Severi T. Functional analysis of bioprosthetic heart valves. Journal of Biomechanics 2005; 38: 1483-1490.

55. Nalla RK, Imbeni V, Kinney JH, Staninec M, Marshall SJ, Ritchie RO. In vitro fatigue behavior of human dentin with implications for life prediction. Journal of Biomedical Materials Research 2003; 66A: 10-20.

56. Huang SM. Fatigue life prediction of a woven fabric composite subjected to biaxial cyclic loads. Composites 2002; A33: 253-266.

57. Mouritz AP. A simple fatigue life model for three-dimensional fiber-polymer composites. Journal of Composite Materials 2006; 40: 455-469.

58. Engelmayr GC, Hildebrand DK, Sutherland FWH, Mayer JE, Sacks MS. A novel bioreactor for the dynamic flexural stimulation of tissue engineered heart valve biomaterials. Biomaterials 2003; 24: 2523-2532.

59. Merryman WD, Huang HS, Schoen FJ, Sacks MS. The effects of cellular contraction on aortic valve leaflet flexural stiffness. Journal of Biomechanics 2006; 39: 88-96.

60. Kim H, Lu J, Sacks MS, Chandran KB. Dynamic simulation of bioprosthetic heart valves using a new finite element shell model based on experimental data. Proceedings of the 2006 Summer Bioengineering Conference, Amelia Island, FL, June 21-25, 2006. 
61. Gao ZB, Pandya S, Hosein N, Sacks MS, Hwang NHC. Bioprosthetic heart valve leaflet motion monitored by dual camera stereo photogrammetry. Journal of Biomechanics 2000; 33: 199-207.

62. Lee B. Reconstruction of trileaflet heart valve leaflet motion using photogrammetry and biquintic finite element method. Master's Thesis 2003. Florida International University: Department of Biomedical Engineering.

63. Sun W, Abad A, Sacks M. Simulated bioprosthetic heart valve deformation under quasi-static loading. Journal of Biomechanical Engineering 2005; 127: 905-914.

64. Thornton MA, Howard IC, Patterson EA. Three-dimensional stress analysis of polypropylene leaflets for prosthetic heart valves. Medical Engineering Physics 1997; 19: 588-597.

65. Bernacca GM, O'Connor B, Williams DF, Wheatley DJ. Hydrodynamic function of polyurethane prosthetic heart valves: influence of Young's modulus and leaflet thickness. Biomaterials 2002; 23: 45-50.

66. Labrosse MR, Beller CJ, Robicsek F, Thubrikar MJ. Geometric modeling of functional trileaflet aortic valves: development and clinical applications. Journal of Biomechanics 2007; Article in Press.

67. Skinner HB. Composite technology for total hip arthroplasty. Clinical Orthopedic Related Research 1988; 235: 224 - 236.

68. Hashin Z. A reinterpretation of the Palmgren-Miner rule for fatigue life prediction. Journal of Applied Mechanics 1980; 47: 324-328.

69. Hashin Z. Failure criteria for unidirectional fiber composites. Journal of Applied Mechanics 1980; 47: 329-334.

70. Hashin A. Fatigue failure criteria for unidirectional fiber composites. Journal of Applied Mechanics 1981; 48: 846-852.

71. Hashin Z. Fatigue failure criteria for combined cyclic stress. International Journal of Fracture 1981; 17: 101-109.

72. Salzman DL, Kleinert LB, Berman SS, Williams SK. Inflammation and neovascularization associated with clinically used vascular prosthetic materials. Cardiovascular Pathology 1999; 8: 63-71.

73. Deck JD, Thubrikar MJ, Schneider PJ, and Nolan SP. Structure, stress, and tissue repair in aortic valve leaflets. Cardiovascular Research 1988; 22: 7-16. 
74. Thubrikar MJ, Skinner JR. Stress analysis of porcine bioprosthetic heart valves in vivo. Journal of Biomedical Materials Research 1982; 16: 811-826.

75. Thubrikar MJ, Piepgrass WC, Deck, JD, and Nolan SP. Stresses on natural versus prosthetic aortic leaflets in vivo. The Annals of Thoracic Surgery 1980; 30: 230-239.

76. Rosenhek R, Binder T, Maurer G, Baumgartner H. Normal values for Doppler echocardiographic assessment of heart valve prostheses. Journal of the American Society of Echocardiography 2003; 16: 1116 - 1127. 
APPENDIX 
Appendix I - Valve Manufacturing Protocols 


\section{A. Chemical Solution Preparation}

\section{Preparation of $15 \%$ SIBS $(8.5 \%$ styrene) in toluene solution}

a. Set up mixer with stirring plate, $250 \mathrm{~mL}$ glass jar with black top and Teflon or foil lid, and stirring magnet.

b. Remove glass bottle and weigh in:

i. $\quad 30 \mathrm{~g}$ of $8.5 \%$ SIBS

ii. Toluene $170 \mathrm{~g}$

c. Mix with or without heat $\left(75^{\circ} \mathrm{C}\right)$ with lid secured until dissolved

\section{Preparation of $15 \%$ SIBS (16\% styrene) in cyclopentane solution}

a. Set up mixer with stirring plate, $60 \mathrm{~mL}$ glass jar with black top and Teflon or foil lid, and stirring magnet.

b. Remove glass bottle and weigh in:

i. $2.1 \mathrm{~g}$ of $16 \%$ SIBS

ii. $\quad 11.9 \mathrm{~g}$ of cyclopentane

c. Mix without heat $\left(75^{\circ} \mathrm{C}\right)$ with lid secured until dissolved.

d. Remove from mixer and weigh in $5.2 \mathrm{~g}$ of isopropanol ( $95 \%$ titration).

i. Mix without heat until dissolved.

\section{B. Fabricating Compression-molded SIBS Stent}

1. Select stent design and SIBS polymer combination for particular valve prototype to be manufactured (Figure 106), and identify specific compression mold.
a. Low profile geometry with $30 \%$ styrene SIBS
b. High profile geometry with $30 \%$ styrene SIBS
c. Medium profile geometry with $48.5 \%$ styrene SIBS

2. Examine all components of compression mold to ensure that they are clean. As needed, smooth mold parts with 320 or finer grit wet/dry sand paper, flush with water, and clean well with isopropyl alcohol and allow to dry completely.

3. Spray all mold parts with Teflon mold release.

4. Block hole at the bottom of the female mold with a piece of rolled-up paper towel and half-fill the mold with SIBS. Material used to block the hole in the female mold should not protrude out of either end of the hole.

5. Set temperature of plates on Pasadena Hydraulic Press to $220^{\circ} \mathrm{C}$.

6. Attach thermocouple to temperature output on the female mold. 
7. Place separated male and female molds on center of compression platform. Do not allow the top platen to touch the mold.

8. Heat molds until temperature output reads $135^{\circ} \mathrm{C}$.

9. Place the male portion of the mold inside the female portion.

10. Close press until it touches, but does not apply pressure on the mold (note: the male portion of the mold will compress into the female portion, but no pressure increase will register on the pressure gauge).

11. Hold in place until the mold reaches $150^{\circ} \mathrm{C}$.

12. Compress to $7000 \mathrm{lb}$.

13. Hold pressure until the mold reaches $180^{\circ} \mathrm{C}$.

14. Remove from press and clamp in hydraulic press or vice. Apply compressive force until resistance is felt. Allow assembly to cool (approximately $15 \mathrm{~min}$ ).

15. Remove the stent from the mold as follows:

a. Invert the assembly in the hydraulic press so that the male portion is facing downwards and the female portion is secure in place preventing any movement.

b. Place a small diameter pin in the hole in the female base (facing up). Apply pressure until the female part separates from the male part.

c. Using a razor blade, trim any flash from the external surface of the male mold, and trim along the edge of the stent to release it from the mold edges for ease of removal.

d. The stent remains attached to the male mold. Ease the stent gently off the mold with forceps making sure not to distort it. Application of isopropanol can aid in this process.

16. Use a razor blade to cut any excess flash from stent and examine stent for imperfections. If imperfections exist, discard stent.

17. Prepare suture holes in the stent as follows:

a. Place the stent on the Delrin mandril of the stent hole alignment tool, aligning the posts with the grooves in the fixture.

b. Place the mandril in the template, aligning the divot in the mandrel with the set screw in the template.

c. Hand-tighten the set screw to lock the mandril in position.

d. Mount a 0.027 " diameter drill bit in the drill press. Set the drill press stop to where the bit touches the Delrin.

e. Place the alignment tool on a V-block for support.

f. Turn on the drill press. 
g. Carefully align the alignment tool under the drill bit and drill through a hole in the template. Use of magnifying loops may help maintain accuracy. Press until the stop.

h. Repeat the previous step, repositioning the alignment tool on the V-block, until all holes are drilled.

i. Turn off the drill. Remove the stent from the alignment tool and inspect to ensure all holes are drilled in the proper positions and are through the entire stent wall. Repeat drilling process as needed.

18. Rinse stent with IPA and allow it to dry.

\section{Leaflet Fabrication}

\section{Pre-formed Individual Leaflets: Design 1}

a. Dip dipping mold into $15 \%$ SIBS (8.5\% styrene)/toluene solution and place in oven at $75^{\circ} \mathrm{C}$ for 30 minutes, then remove and cool.

b. Once mold has cooled to room temperature, place PET mesh (LARS®) over coated mold with maximum elongation oriented circumferentially.

c. Use O-ring to secure knit to dipping mold, making sure that mesh does not bunch.

d. Dip assembly in $15 \%$ SIBS (8.5\% styrene)/toluene solution.

e. Place in oven and dry at $75^{\circ} \mathrm{C}$ for $30 \mathrm{~min}$, then remove and cool.

f. Once mold has cooled, dip again in the same solution and dry for $>2$ hours.

g. Cool and dip in soapy water (makes it less tacky).

h. Remove from mold (use soapy water where necessary).

i. Wash off soap and dry

\section{Dip-Coated Cylinder Leaflets: Design 2}

a. Select particular fabric for use.

b. Identify direction of minimum and maximum elongation on fabric, and cut into a 3" x 3" square parallel to the max and min elongation respectively

c. Wrap the cut fabric tightly around the mandril made for heat sealing with the maximum stretch oriented in either the circumferential or radial direction, dependent on the specific prototype.

d. Heat-seal the fabric into its cylindrical form and cut of any excess.

e. Secure one free edge of the fabric to a cylindrical dipping mandril.

f. Slowly dip the fabric into the SIBS solution specific for the valve prototype (A.1 or A.2) making sure not to entrap air bubbles or collapse the cylinder.

g. Dry in an oven set to approximately $60^{\circ} \mathrm{C}$ for 30 minutes 
h. Repeat steps C.2.f - C.2.g once more to form a 2-dip coated cylinder

i. Neaten the edges of the cylinder by cutting off any excess or uncoated material.

\section{Compression-Molded Cylinder Leaflets: Design 3a}

a. Manufacture of isotropic SIBS cylinder 1:

i. Using an $8.5 \%$ styrene extruded SIBS ring, slide a cut portion (approximately $3-5 \mathrm{~mm}$ in length) onto the center of the cylinder insert (component of the leaflet compression mold).

ii. Place the cylinder insert into the small female clam shell, and compression mold it on the Pasadena Press (set to $200^{\circ} \mathrm{C}$ ).

- Close the press until it is at its tightest, un-pressurized point, and hold for $1 \mathrm{~min}$.

- Add 30,000 lbs of pressure, and hold for $30 \mathrm{sec}$.

- Remove from press and clamp in vice until cool.

iii. Very delicately separate the two sides of the clam shell so as not to disturb the underlying polymer.

- Ensure that the polymer is only attached to one side of the clam shell.

iv. Under a microscope, trim the cylinder to size by removing all flash at the top, bottom, and both sides of the cylinder insert.

v. Do not remove the polymer from the cylinder insert, but remove the caps on the insert to clean out any flash trapped inside.

b. Forming First Laminate with the Dacron Cylinder:

i. Only circumferentially oriented JSI Dacron knit leaflets were manufactured using this method.

ii. Identify direction of minimum and maximum elongation on Dacron, and cut into a 3" x 3 " square parallel to the max and min elongation respectively

iii. Wrap the cut fabric tightly around the mandril made for heat sealing with the maximum stretch oriented in the circumferential direction.

iv. Heat-seal the fabric into its cylindrical form and cut of any excess.

v. Slide heat sealed Dacron on top of the SIBS cylinder manufactured in procedure C.3.a.

- Orient heat seal along one of the flash/joint lines.

vi. Slide Teflon ${ }^{\circledR}$ shrink tubing over the top, and use a heat gun to shrink it to size. 
vii. Place the assembly in the larger clam shell and compression mold it on the Pasadena Press set to $200^{\circ} \mathrm{C}$.

- Close the press until it is at its tightest, un-pressurized point, and hold for $1.5 \mathrm{~min}$.

- Add 30,000 lbs of pressure, and hold for $30 \mathrm{sec}$.

- Remove from press and clamp in vice until cool.

viii. Separate the two sides of the clam shell.

ix. Remove the shrink tubing.

x. Remove the first Dacron/SIBS laminate by liberally applying isopropanol, and ease the cylinder off by rolling it inside-out.

xi. Dry in an oven at $80^{\circ} \mathrm{C}$ for 15 mins.

c. Manufacture of individual SIBS cylinder 2:

i. Repeat procedure C.3.a.

d. Forming Final Compression-Molded SIBS/Dacron Laminate:

i. Ease first laminate manufactured in procedure C.3.b onto the top of the individual SIBS cylinder manufactured in procedure C.3.c.

- IPA may be required in this process. If used, dry for 15 mins in an oven set to $80^{\circ} \mathrm{C}$.

ii. Repeat procedures C.3.b.v - C.3.b.xi.

\section{Hybrid Cylinder Leaflets: Design 3b}

a. Only circumferentially oriented JSI Dacron knit leaflets were manufactured using this method.

b. Identify direction of minimum and maximum elongation of fabric.

c. Cut fabric into 3.0 " x 3.0 " rectangle parallel to min and max elongation respectively.

d. Place one edge of the fabric (the direction of minimum elongation) between the Delrin heat seal mandrils. Wrap the fabric tightly around the mandrils ensuring that the maximum stretch is oriented circumferentially.

i. Make sure not to stretch the fabric, simply ensure that it is a tight fit.

e. Place the fabric on the heat sealer platform with the region to be sealed adjacent to the ribbon.

f. Confirm the variable autotransformer is set to the correct position (an ink line on the transformer indicates the proper setting). Do not exceed this voltage or the fabric will burn.

g. Lower the upper portion of the heat sealer to compress the fabric against the wire. 
h. Press the red button on the timer control to heat-seal the fabric (set to $7 \mathrm{~s}$ ).

i. Cut off excess fabric on both the outside and inside of the heat-sealed cylinder (note: requires inverting it).

j. Dip coat the fabric cylinder

i. Stir $15 \%$ SIBS $(8.5 \%$ styrene $) /$ toluene solution for five minutes on magnetic stirrer.

ii. Secure one edge of the heat-sealed Dacron cylinder to the Dipping/Attachment Mandril with scotch tape.

iii. Slowly dip approximately $2 / 3^{\text {rds }}$ of the length of the fabric into the SIBS solution. Retract slowly from the solution. Do not allow the fabric to touch either the sides or bottom of the solution container. Prevent any air bubbles from coming into contact with the dipped fabric. If the opening at the bottom of the tube closes, discard this sample and start again.

iv. Leave dipped fabric on mandril in the fume hood for 10 minutes.

v. Transfer to and dry in an oven set to approximately $80^{\circ} \mathrm{C}$ for 20 minutes.

vi. Remove cylinder from Dipping/Attachment Mandril, invert it, and secure it to the mandrel as before. It may be helpful to place the cylinder on the mandrel to facilitate inversion.

vii. Repeat step C.4.j.i - C.4.j.v.

viii. Trim the edges of the cylinder using the circular razor so as to remove any rough edges and uncoated fabric.

k. Compression-mold the coated fabric cylinder.

i. Slide the cylinder onto the cylinder insert of the leaflet compressionmolding tool.

ii. Slide Teflon ${ }^{\circledR}$ shrink tubing over the assembly, and use heat gun (set to \#4 on dial) to shrink the tubing onto the cylinder. Mount the assembly on a drill and rotate the assembly at low speed while shrinking the tubing.

iii. Place the assembly in the clam shell mold and compress it on the Pasadena Press set to $200^{\circ} \mathrm{C}$.

- Close the press to its tightest, un-pressurized point for $1.5 \mathrm{~min}$.

- Compress to 3,000 lb for $30 \mathrm{~s}$.

- Remove the assembly from the press and clamp it in a vice until cool ( $\sim 15$ mins $)$.

iv. Separate the two sides of the clam shell.

v. Remove the shrink tubing. This is most easily accomplished by making a small, diagonal slit, gripping the free segment, and peeling the tubing off in a spiral. 


\section{Solvent-Cast Cylinder leaflets: Design 4}

a. Clean the solvent casting plate to ensure no polymer residue is present.

i. Wash in warm soapy water.

ii. Rinse with deionized water and allow to dry

b. Cut an aluminum foil gasket following the dimensions of the top plate of the solvent casting plate and secure it in place on the bottom plate of the solvent casting plate.

c. Cut reinforcement fabric into a 3 " by 3 " square parallel to the max and min elongation respectively

d. Place reinforcement fabric in position on top of the bottom casting plate and gasket and secure with tape along the outside edges.

e. Secure the top plate with the 10-32 bolts to clamp the reinforcement fabric between the two plates.

i. Make sure there is no rippling in the fabric.

f. Level the plate on a flat surface by adjusting the four corner $1 / 4-20$ bolts.

g. Using a pipette, slowly dispense $11 \mathrm{~mL}$ of the $15 \%$ SIBS $(8.5 \%$ styrene) in toluene solution into the solvent casting plate

i. Make sure no bubbles form on pouring and make sure the entire surface of the reinforcement fabric is coated.

h. Cover the casting plate with a glass bowl for 24 hours to reduce air flow over the SIBS solution and reduce the rate of evaporation

i. After 24 hours lift one edge of the glass bowl $1 \mathrm{~cm}$ to allow minor exchange of air for an additional 24 hours

j. Transfer solvent casting plate to oven and dry for 24 hours at $60^{\circ} \mathrm{C}$.

k. Remove plate from oven and allow to cool to room temperature.

1. Dismantle solvent casting plate and remove composite SIBS/reinforcement sheet.

m. Heat seal sheet into cylinder:

i. Place one edge of the fabric (the direction of minimum elongation) between the Delrin heat seal mandrils. Wrap the fabric tightly around the mandrils ensuring that the maximum stretch is oriented circumferentially.

- Make sure not to stretch the fabric, simply ensure that it is a tight fit.

ii. Place the fabric on the heat sealer platform with the region to be sealed adjacent to the ribbon. 
iii. Confirm the variable autotransformer is set to the correct position (an ink line on the transformer indicates the proper setting). Do not exceed this voltage or the fabric will burn.

iv. Lower the upper portion of the heat sealer to compress the fabric against the wire.

v. Press the red button on the timer control to heat-seal the fabric (set to $7 \mathrm{~s}$ ).

vi. Cut off excess fabric on both the outside and inside of the heat-sealed cylinder (note: requires inverting it).

\section{Valve Assembly}

\section{Preformed Individual Leaflets: Design 1}

a. Position leaflet on placement mandril

b. Using syringe filled with $15 \%$ SIBS in Toluene, paint area of contact on both the leaflet and the stent with SIBS solution.

c. Bond leaflet to stent making sure to orient correctly

d. Dry in oven for $15 \mathrm{~min}$ at $75^{\circ} \mathrm{C}$.

e. Repeat with other 2 leaflets

i. In order to prevent leaflets bonding together, separate each with aluminum foil.

f. Ensure leaflets touch as required

g. Trim leaflet coaptation area such that slope ends halfway down flats.

h. Thermoform leaflets closed, if necessary.

i. Store in the labeled zip-lock bag that contained the stents

i. Record lot numbers of leaflets and stents

\section{Cylinder Valve: Designs 2-4}

a. This procedure is used for the manufacture of all cylinder valves, including the leaflet cylinders produced in C. 2 - C.5 attached to either the high profile geometry $(30 \%$ styrene SIBS) or medium profile geometry $(48.5 \%$ styrene SIBS).

b. Preliminary attachment of the leaflet cylinder to the internal surface of the stent.

i. Slide the SIBS-coated valve cylinder over the Dipping/Attachment mandril. Place enough fabric onto the mandrel to cover the entire height of the stent.

ii. Position the stent over the fabric (top of the posts first), aligning one stent post with the heat-seal joint. 
iii. Using the $15 \%$ SIBS ( $8.5 \%$ styrene)/toluene solution, bond the cylinder to the stent.

- Place a small volume of the solution (approximately $0.1 \mathrm{~mL}$ ) into a $1 \mathrm{ml}$ syringe with a blunt 20 gage needle.

- Dispense a thin coat of the solution on the leaflet cylinder along the curves of the stent base between the stent posts (but not up the edges of the stent posts themselves).

- Slide the stent over the coated fabric, orienting it so that the heatsealed joint runs along one stent post. Leave a gap of approximately 1 $\mathrm{mm}$ between the top of the posts and the exposed fabric above.

- Dispense a thin coat of the solution along the bottom ring of the stent.

- Dry in the oven set to $80^{\circ} \mathrm{C}$ for 15 minutes.

c. Suturing the fabric to the stent

i. Sew the cylinder to the stent using the 5-0 Ethibond Excel green braided polyester suture. Start at the bottom of a post and suture in through the assembly and back out through the next hole up the post.

ii. Knot the suture at the base of the stent post, on the outside of the assembly, using a square knot (black arrows). Do not trim the suture ends at this point.

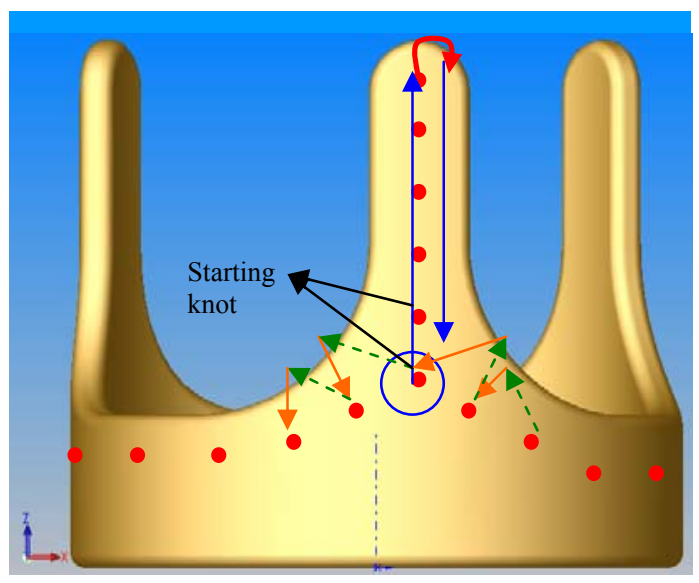

\section{Suturing Pattern}

i. With the free end of the suture line, continue suturing all the way up the post by sewing in and out the stent at each successive hole (blue arrow).

ii. Once the top hole is reached, suture up and over the post, looping around the top of the post and entering through the leaflet fabric (red arrow). 
iii. Suture back though the post through the top hole. Repeat this procedure a second time.

iv. Reverse the suturing direction and come back down the post through the same holes in the stent (blue arrow).

v. At base of post, suture out through leaflet (green arrow) adjacent to the first hole along the curve. Suture in (orange arrow) through the adjacent hole.

vi. Continue this pattern around the entire stent. Along the curves, stay as close to the stent as possible when suturing through the leaflet, but do not pierce the stent. Keep stitches evenly spaced and approximately perpendicular to the curve of the stent.

vii. Knot off using three single overhand knots at the end by using starting knot/suture end.

viii. Trim the remaining suture line (both ends) to approximately $1 \mathrm{~mm}$ in length.

d. Replace the stent/valve cylinder assembly on the Dipping/Attachment mandril, and use blunt tip forceps to fold the excess fabric up onto the outside of the assembly. Make sure to pull the material tight so that the cylinder that forms the leaflets covers the outside of the stent with a single layer.

e. Trim the excess external leaflet material to approximately $1 \mathrm{~mm}$ above the height of the leaflets. Be careful not to cut the leaflets themselves.

f. Using the $15 \%$ SIBS ( $8.5 \%$ styrene)/toluene solution, bond the cylinder to the external portion of the stent by dispensing a thin coat of the solution along the contact regions (i.e., where the outer fabric layer contacts the underlying stent) with a syringe. It may help to create a uniform coating by dispensing a small amount of coating then smearing it with the syringe barrel.

g. Dry in an oven set to $80^{\circ} \mathrm{C}$ for $15 \mathrm{~min}$.

h. Use scissors to cut the excess fabric so the external cover follows the contours of the stent. Do not damage the inner layer of fabric with the scissors tips. Be very careful not to cut through both layers of fabric or to cut the sutures.

i. Add one reinforcing suture at the top of each of the stent posts to secure the external fabric. Suture in top hole and out the next hole down and knot off with a triple overhand knot on the outside of the assembly. Trim the suture ends to approximately $1 \mathrm{~mm}$ long.

j. Forming the Leaflets

i. Place the assembly in the leaflet forming fixture with the stent posts aligned with the spaces between the forming tools and the aluminum leaflet forming fixtures in their grooves. Confirm the valve is well seated in the ring. 
ii. Mount the compression ring over the valve. Screw the ring to slip fit over the valve.

iii. Place the shim stock tools between each pair of adjacent leaflets to prevent the adjacent leaflets from touching.

iv. Turn the cam to its maximum compressing point to press the leaflet forming fixtures into the valve leaflets. The three leaflets will come together to form the valve shape. Confirm symmetry and proper alignment.

v. Tighten the compression ring. Confirm all parts are well seated and aligned.

vi. Place in the oven set to $80^{\circ} \mathrm{C}$ for 1 hour to allow the leaflets to assume their shape.

k. Cut to shape so that each leaflet joins in the center of the valve and creates a smooth contour. 
Appendix II - Stent Drawings 


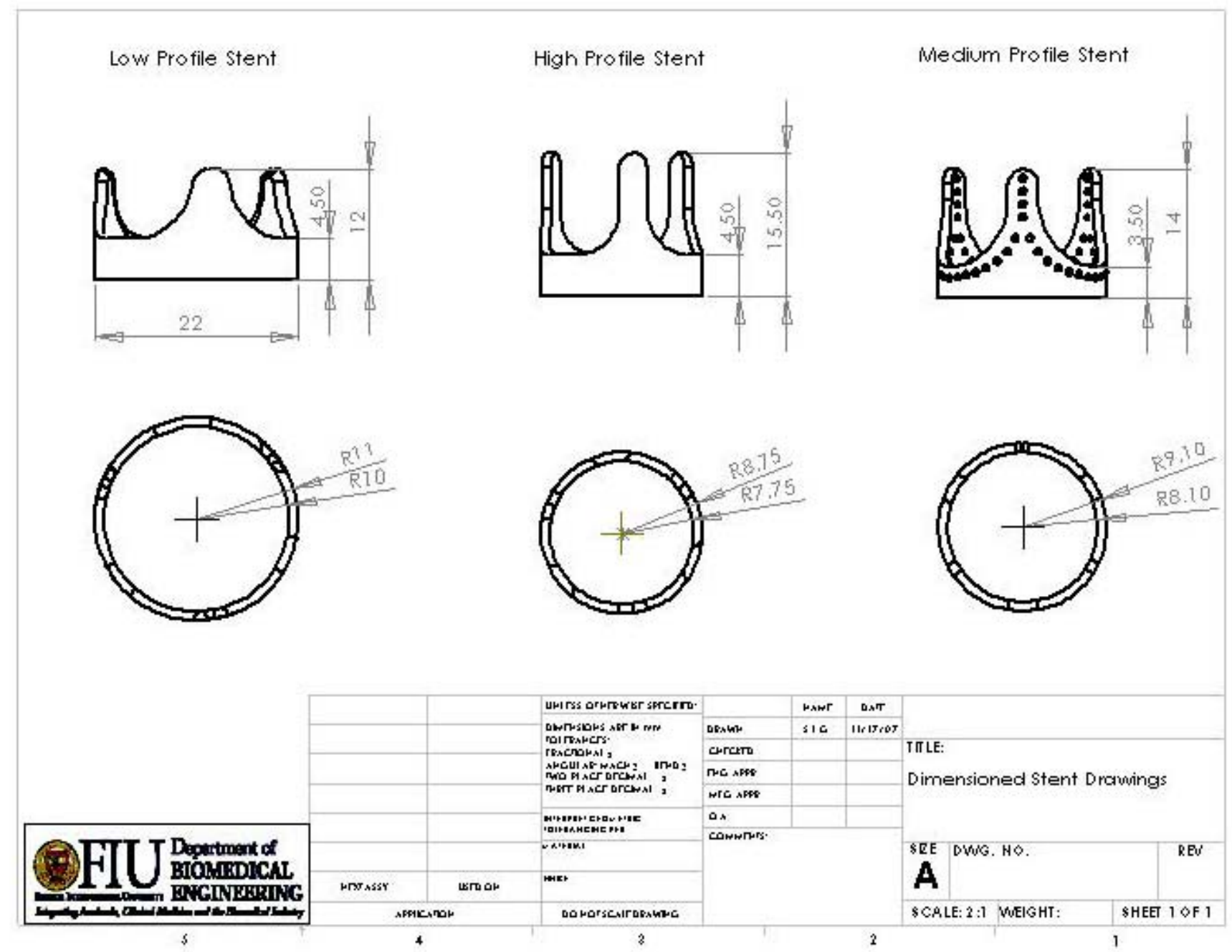

Figure 106. Dimensioned drawings of low profile, high profile, and medium profile stents. 
Appendix III - Durability Rating 
Table 18. Qualitative valve failure rating

\begin{tabular}{|c|c|c|c|c|c|c|c|c|c|}
\hline Rating & $\begin{array}{c}\text { Surface } \\
\text { Coverage }\end{array}$ & Holes & Tears & Fraying & $\begin{array}{l}\text { Incomplete } \\
\text { coaptation }\end{array}$ & Delamination $^{1}$ & $\begin{array}{c}\text { Excessive } \\
\text { Deformation }\end{array}$ & $\begin{array}{c}\text { Other } \\
\text { Mechanical } \\
\text { Breakdown } \\
\end{array}$ & $\begin{array}{c}\Delta P \text { from Vivitro } \\
\text { HiCycle (mmHg) }\end{array}$ \\
\hline 0 & none & none & none & none & none & none & none & none & $90+20 /-0$ \\
\hline 1 & none & Pin holes & none & $\begin{array}{l}\text { Slight fraying } \\
\text { of sutures and } \\
\text { edges }\end{array}$ & $\begin{array}{l}\text { Minimal amount } \\
\text { that doesn't affect } \\
\text { valve function }\end{array}$ & none & none & & $90+20 /-0$ \\
\hline 2 & $\begin{array}{l}\text { Polymer surface } \\
\text { cracking and } \\
\text { imperfections as } \\
\text { is visible under } \\
\text { the microscope }\end{array}$ & $\begin{array}{l}\text { Enlargement of } \\
\text { pin holes }\end{array}$ & $\begin{array}{l}\text { Small tears } \\
\text { not affecting } \\
\text { valve } \\
\text { function }\end{array}$ & $\begin{array}{l}\text { Increase in } \\
\text { edge and } \\
\text { suture fraying } \\
\text { not resulting } \\
\text { in change in } \\
\text { function }\end{array}$ & $\begin{array}{l}\text { Possible incomplete } \\
\text { closure not having a } \\
\text { marked impact on } \\
\text { valve function }\end{array}$ & none & none & none & $90+20 /-0$ \\
\hline 3 & $\begin{array}{l}\text { Polymer surface } \\
\text { cracking and } \\
\text { imperfections } \\
\text { resulting in } \\
\text { exposure of } \\
\text { underlying } \\
\text { fabric or } \\
\text { tears/holes } \\
\end{array}$ & $\begin{array}{l}\text { Pin holes } \\
\text { visible with } \\
\text { stroboscope } \\
\text { using leaflet } \\
\text { motion }\end{array}$ & $\begin{array}{l}\text { Small tears } \\
\text { visible with } \\
\text { stroboscope }\end{array}$ & $\begin{array}{l}\text { Increase in } \\
\text { fraying } \\
\text { resulting in } \\
\text { minor } \\
\text { delamination } \\
\text { and rough } \\
\text { edges }\end{array}$ & $\begin{array}{l}\text { Noticeable decrease } \\
\text { in valve coaptation. } \\
\text { Insufficient closure } \\
\text { postulated to result } \\
\text { in increased } \\
\text { regurgitation }\end{array}$ & $\begin{array}{l}\text { At tops of stent } \\
\text { posts only }\end{array}$ & $\begin{array}{l}\text { Possible } \\
\text { ballooning of } \\
\text { leaflets at the base }\end{array}$ & $\begin{array}{l}\text { Any other factor } \\
\text { contributing to } \\
\text { insufficient } \\
\text { opening and/or } \\
\text { closing of the } \\
\text { valve }\end{array}$ & $90+20 /-0$ \\
\hline 4 & $\begin{array}{l}\text { Further } \\
\text { breakdown } \\
\text { notably } \\
\text { affecting valve } \\
\text { function }\end{array}$ & $\begin{array}{l}\text { Holes visible } \\
\text { to the naked } \\
\text { eye }\end{array}$ & $\begin{array}{l}\text { Tears visible } \\
\text { to the naked } \\
\text { eye }\end{array}$ & $\begin{array}{l}\text { Fraying } \\
\text { resulting in } \\
\text { fracture }\end{array}$ & $\begin{array}{l}\text { One or more leaflets } \\
\text { motion restricted } \\
\text { causing improper } \\
\text { closure }\end{array}$ & $\begin{array}{l}\text { Propagation of } \\
\text { delamination } \\
\text { down the posts }\end{array}$ & $\begin{array}{l}\text { Of leaflets and/or } \\
\text { stent }\end{array}$ & $\begin{array}{l}\text { Resulting in } \\
\text { restriction in } \\
\text { function }\end{array}$ & $90+20 /-0$ \\
\hline 5 & $\begin{array}{l}\text { Complete } \\
\text { breakdown of } \\
\text { polymer coating }\end{array}$ & $\begin{array}{l}\text { Large holes } \\
\text { easily visible } \\
\text { during cycling }\end{array}$ & $\begin{array}{l}\text { Large tears } \\
\text { and crack } \\
\text { propagation }\end{array}$ & $\begin{array}{l}\text { Complete } \\
\text { fracture }\end{array}$ & $\begin{array}{l}\text { Improper coaptation } \\
\text { and possible } \\
\text { prolapse }\end{array}$ & $\begin{array}{l}\text { Greater than } \\
90 \% \\
\text { delaminating of } \\
1 / \text { more leaflets }\end{array}$ & $\begin{array}{l}\text { Preventing valve } \\
\text { function }\end{array}$ & $\begin{array}{l}\text { Anything } \\
\text { contributing to } \\
\text { complete loss of } \\
\text { function }\end{array}$ & $<<90+20 /-0$ \\
\hline
\end{tabular}

${ }^{1}$ Extent of delamination is determined by gently tugging on each leaflet with a pair of forceps, making sure not to damager the leaflet in the process 
Appendix IV - Photogrammetry Results 


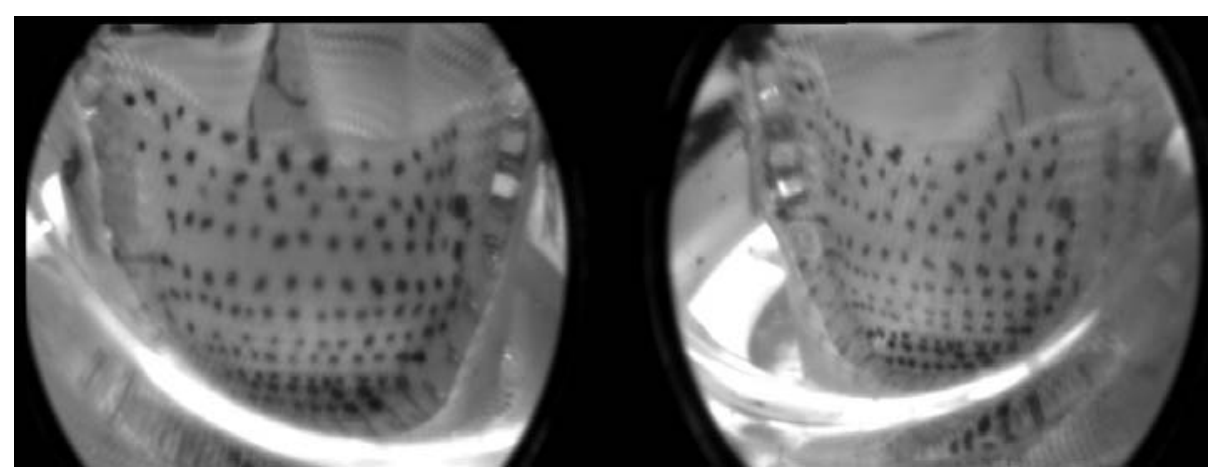

Figure 107. Photogrammetry image pairs corresponding to Frame 1 in Figure 66.

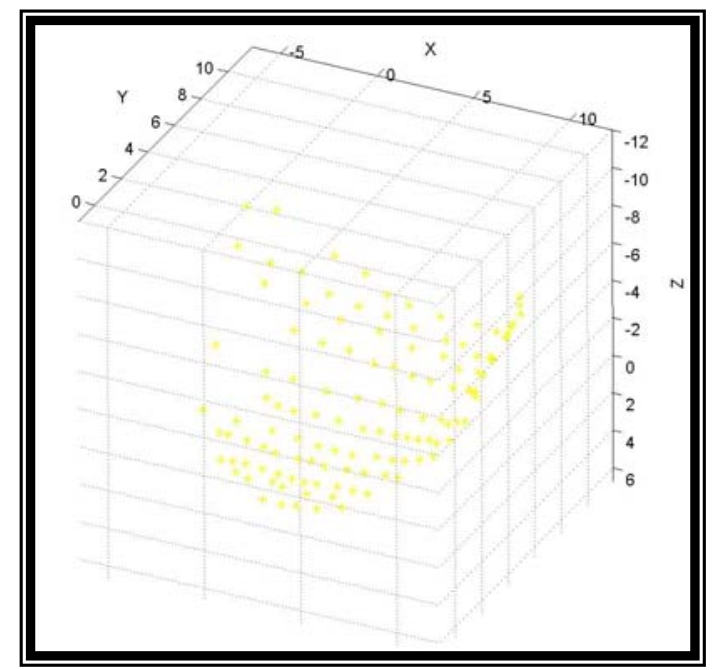

Figure 108. MatLab regeneration of fiducial points from the $\mathrm{x}, \mathrm{y}$, and $\mathrm{z}$ coordinated generated in Photomodeler ${ }^{\circledR}$ from Figure 107.

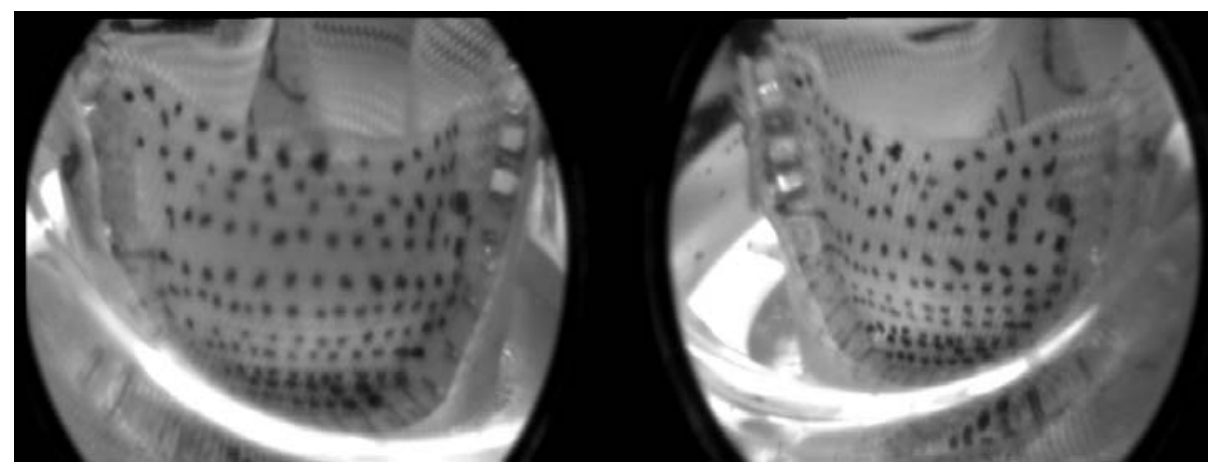

Figure 109. Photogrammetry image pairs corresponding to Frame 2 in Figure 66. 


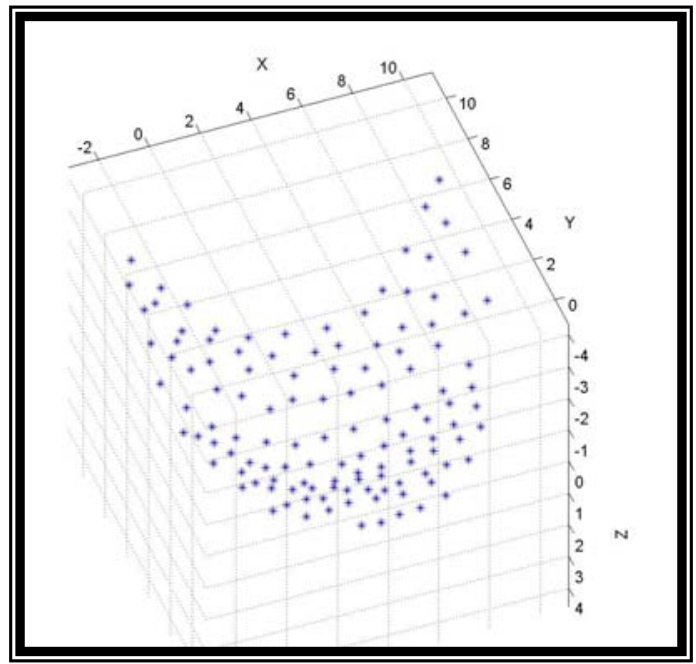

Figure 110. MatLab regeneration of fiducial points from the $x, y$, and $z$ coordinated generated in Photomodeler ${ }^{\circledR}$ from Figure 109.

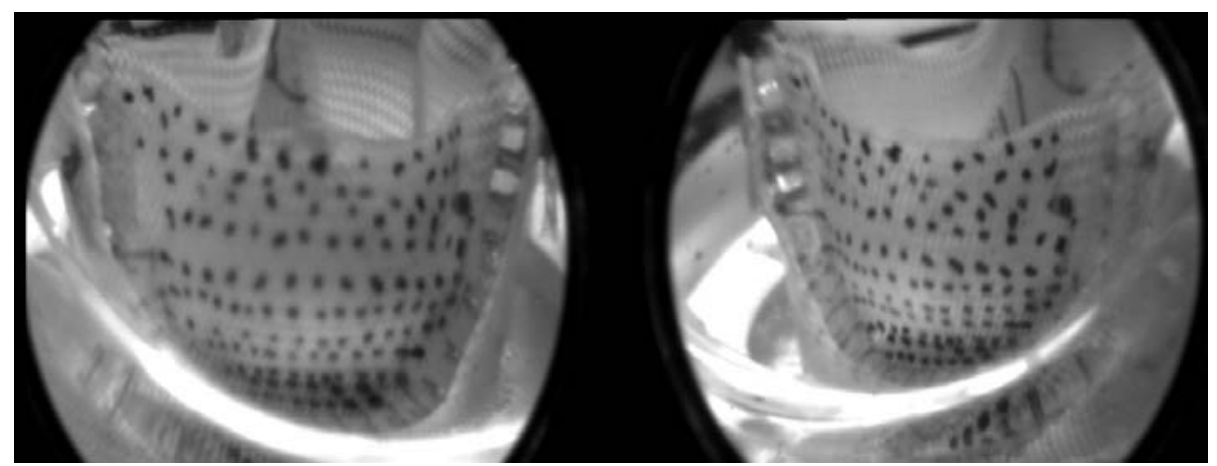

Figure 111. Photogrammetry image pairs corresponding to Frame 3 in Figure 66.

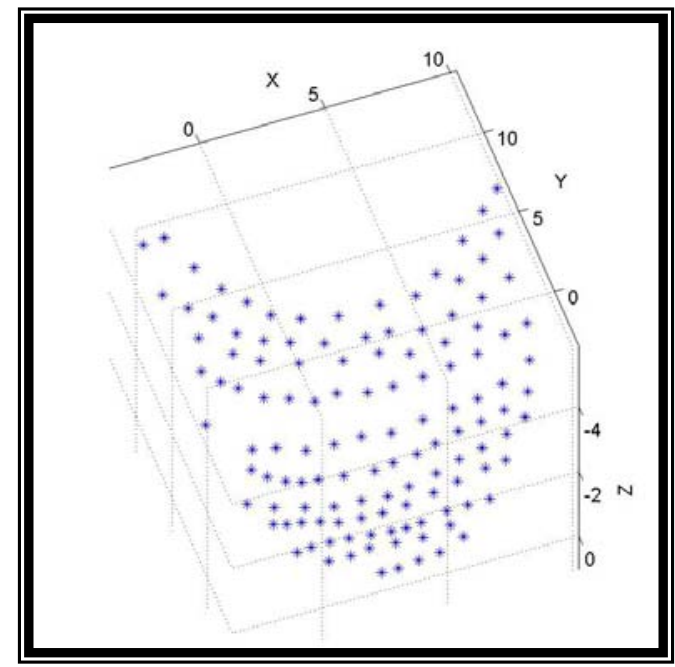

Figure 112. MatLab regeneration of fiducial points from the $\mathrm{x}, \mathrm{y}$, and $\mathrm{z}$ coordinated generated in Photomodeler® from Figure 111. 


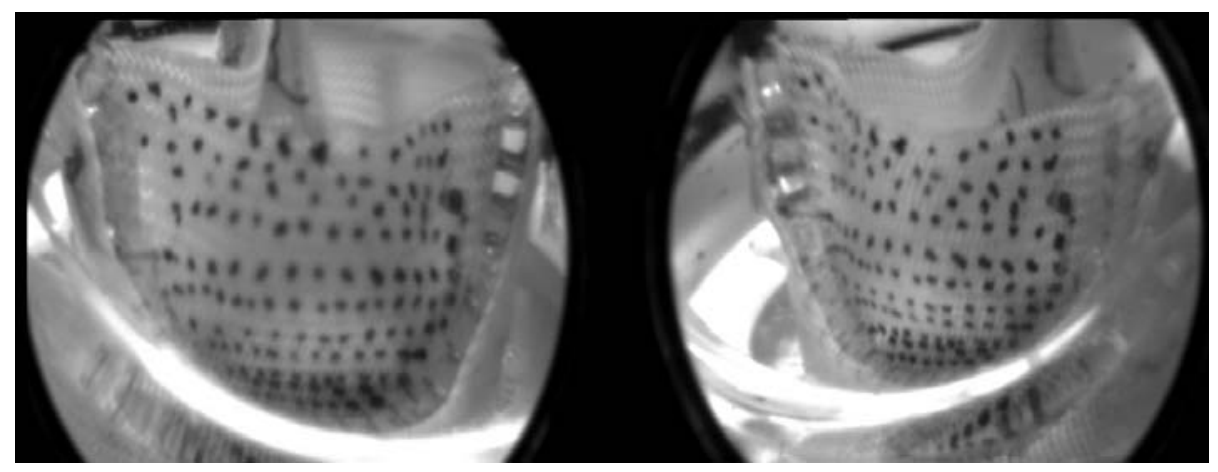

Figure 113. Photogrammetry image pairs corresponding to Frame 4 in Figure 66.

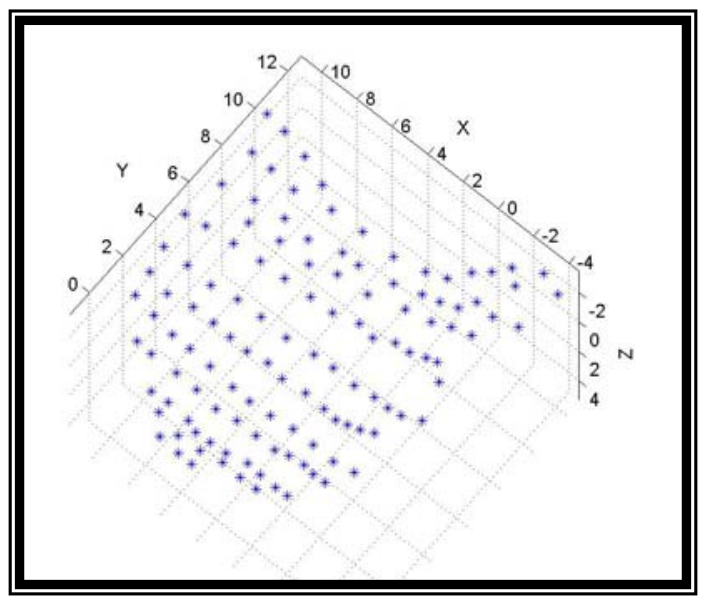

Figure 114. MatLab regeneration of fiducial points from the $\mathrm{x}, \mathrm{y}$, and $\mathrm{z}$ coordinated generated in Photomodeler ${ }^{\circledR}$ from Figure 113.

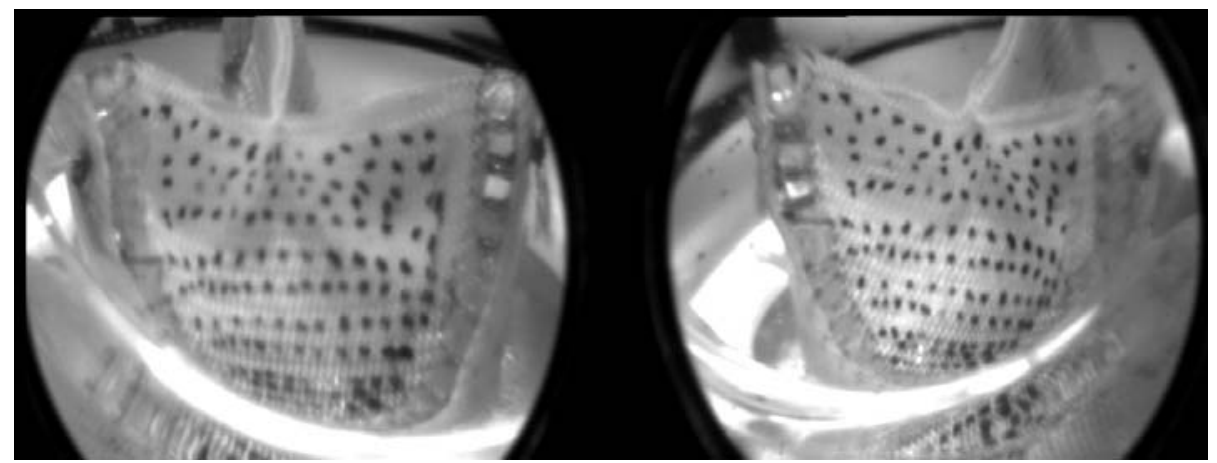

Figure 115. Photogrammetry image pairs corresponding to Frame 6 in Figure 66. 


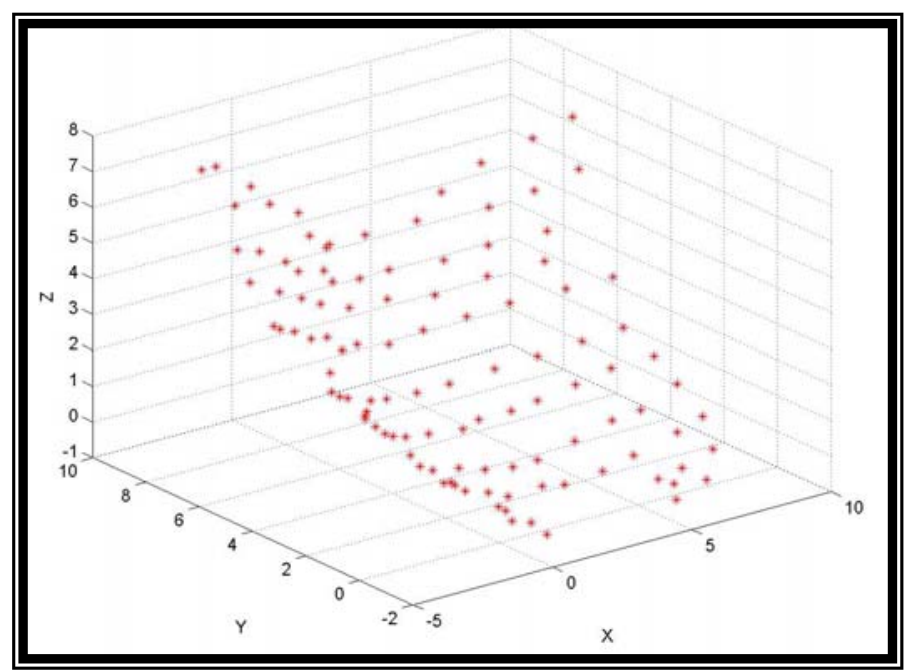

Figure 116. MatLab regeneration of fiducial points from the $\mathrm{x}, \mathrm{y}$, and $\mathrm{z}$ coordinated generated in Photomodeler ${ }^{\circledR}$ from Figure 115.

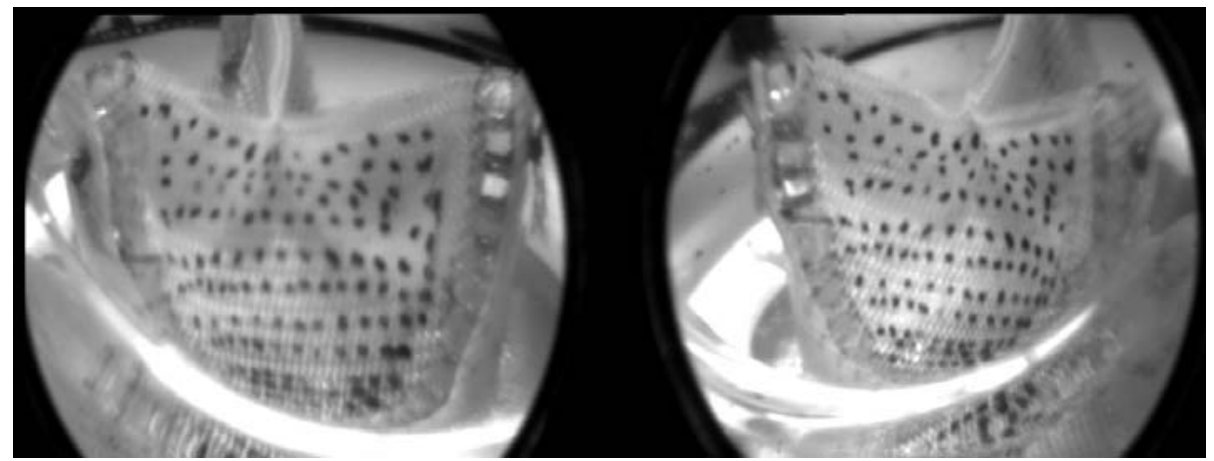

Figure 117. Photogrammetry image pairs corresponding to Frame 8 in Figure 66.

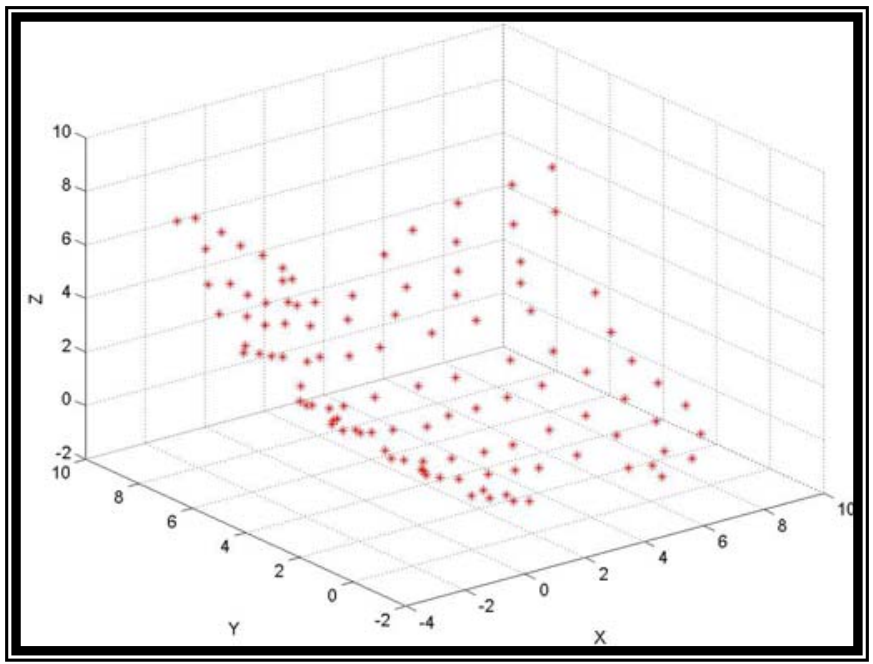

Figure 118. MatLab regeneration of fiducial points from the $\mathrm{x}, \mathrm{y}$, and $\mathrm{z}$ coordinated generated in Photomodeler® from Figure 117. 
VITA

\section{SIOBHAIN LYNN GALLOCHER}

November 24, 1977

2000-2002

2002

2002

2002-2004

2004

2004

2004-2007
Born, Durban, South Africa

Undergraduate Research Assistant

Florida International University

Miami, Florida

B.S. Chemical Engineering

Minor: Biomedical Engineering

Florida International University

Miami, Florida

Outstanding Graduate in Chemical Engineering Award

Graduate Research Assistant

Florida International University

Miami, Florida

M.S. Biomedical Engineering

Florida International University

Miami, Florida

Outstanding Master's Graduate Award

Graduate Research Assistant

Florida International University

Miami, Florida

Inducted into Sigma Xi

PRESENTATIONS AND PUBLICATIONS

Gallocher S, Pinchuk L, Schoephoerster RT. A Comparative Study of Platelet Deposition and Platelet Activation Potential for a Novel Polymer Heart Valve. Proceedings of the 2004 European Society of Biomechanics, 's-Hertogenbosch, The Netherlands, July, 4-7, 2004. 
Gallocher SL, Aguirre AF, Kasyanov V, Pinchuk L, Schoephoerster RT. Durability assessment of a novel polymer trileaflet heart valve. Proceedings of the First International Conference on Mechanics of Biomaterials \& Tissues, Waikoloa Beach Marriott Resort, Hawai'i, USA, 11 - 15 December 2005.

Gallocher SL, Wang Q, Mathison M, Kato YP, Pinchuk L, Schoephoerster RT. Feasibility analysis of the safety and durability of a polymer trileaflet heart valve. Proceedings of the 2006 Summer Bioengineering Conference, Amelia Island, FL, June $21-25,2006$.

Gallocher SL, Aguirre AF, Kasyanov V, Pinchuk L, Schoephoerster RT. A novel polymer for potential use in a trileaflet heart valve. Journal of Biomedical Materials Research B: Applied Biomaterials 2006; 79: 325-334.

Schoephoerster RT, Gallocher S, Pinchuk L, and Kasyanov VA. A novel trileaflet synthetic heart valve. Proceedings of the 2001 ASME IMECE, New York, New York, November, 11-16, 2001.

Schoephoerster RT, Gallocher S, Aguirre A, Pinchuk L, and Kasyanov V. A Polymer Composite Trileaflet Aortic Heart Valve. Proceedings of the 6th Annual Hilton Head Workshop: Prosthetic Heart Valves, Hilton Head Island, SC, March 6-10, 2002.

Schoephoerster RT, Gallocher S, Wang Q, Jaramillo F, Kato YP, Pinchuk L. Polymer valve with potential for minimally invasive and transcatheter delivery. Proceedings of the 10th Annual Hilton Head Workshop: Prosthetic Heart Valves, Hilton Head Island, SC, March 1-5, 2006.

Wang Q, Mathison M, Gallocher S, Jaramillo F, Pinchuk L, Schoephoerster RT. Feasibility analysis of a stent-valve combination device: in vivo evaluation of the novel polymer valve and in vitro evaluation of an alternative fixation method. Proceedings of the 2005 BMES Annual Fall Meeting, Baltimore, MD, September 28 - October 1, 2005.

Wang Q, Gallocher S, Mathison M, Kato Y, Pinchuk L, Schoephoerster RT. In vivo preclinical evaluation of a novel polymeric aortic valve. Proceedings of the 2006 BMES Annual Fall Meeting, Chicago, IL, October 11 - 14, 2006.

Yin W, Gallocher S, Pinchuk L, Schoephoerster RT, Jesty J, Bluestein D. Flow-induced Platelet Activation in a MHV, a tissue valve, and a polymeric valve. Proceedings of the 2004 BMES Annual Fall Meeting, Philadelphia, PA, October 13 - 16, 2005.

Yin W, Gallocher S, Pinchuk L, Schoephoerster RT, Jesty J, Bluestein D. Flow-induced Platelet Activation in a St. Jude Mechanical Heart Valve, a Trileaflet Polymeric Heart Valve, and a St. Jude Tissue Valve. Artificial Organs 2005; 29: 826-831. 